\title{
Economic evaluation of cancer treatments
}

Carin A. Uyl-de Groot 

Economic evaluation of cancer treatments

Economische evaluatie van kankerbehandelingen

Proefschrift

ter verkrijging van de graad van doctor

aan de Erasmus Universiteit Rotterdam

op gezag van de Rector Magnificus

Prof.dr. P.W.C. Akkermans M.A.

en volgens besluit van het College voor Promoties.

De openbare verdediging zal plaatsvinden op woensdag 20 september 1995 om 13.45 uur

door

Cornelia Aaltje Uyl-de Groot

geboren te Oud-Beijerland 
Promotiecommissie:

$\begin{array}{ll}\text { Promotor: } & \text { Prof.dr. F.F.H. Rutten } \\ \text { Overige leden: } & \text { Dr. N.K. Aaronson } \\ & \text { Prof.dr. B. Jönsson } \\ & \text { Prof.dr. B. Löwenberg }\end{array}$

CIP-GEGEVENS KONINKLIJKE BIBLIOTHEEK, DEN HAAG

Uyl-de Groot, Cornelia Aaltje

Economic evaluation of cancer treatments / Cornelia Aaltje

Uyl-de Groot. - [S.1.: s.n.]. - I11.

Thesis Erasmus Universiteit Rotterdam. - With ref. -

With summary in Dutch.

ISBN 90-9008528-9

NUGI 743

Subject headings: cancer treatments; economic aspects/ quality of life

(- C.A. Uyl-de Groot

Photography: False-colour scanning electron micrograph (SEM) of the endothelium lining, the inner surface of blood vessels. Prof. P. Motta, Dept. of Anatomy, University 'La Sapienza', Rome / Science Photo Library, Benelux Press.

Sponsor: Amgen b.v., Breda

Printed by: Ridderprint, Ridderkerk 
To Marco

To my parents 
Carin A. Uyl-de Groot was born on January 26, 1966, in Oud-Beijerland. After following secondary school in Oud-Beijerland, she studied Health Care Policy and Management at the Erasmus University Rotterdam (1985-1990). From March 1990 onwards she is working at the institute for Medical Technology Assessment / Department of Health Care Policy and Management at the Erasmus University Rotterdam. Her research activities are focused on economic evaluation of cancer treatments. 


\section{Contents}

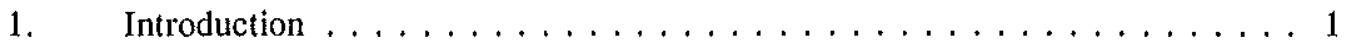

1.1 Cancer and economic evaluation $\ldots \ldots \ldots \ldots \ldots \ldots$

1.2 Cancer and new treatment modalities $\ldots \ldots \ldots \ldots \ldots \ldots$

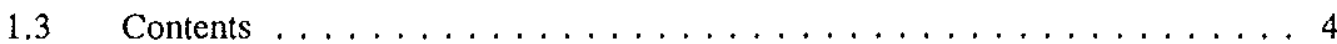

Part I Methodological and practical issues

2. Cost-effectiveness in cancer care $\ldots \ldots \ldots \ldots \ldots \ldots \ldots$

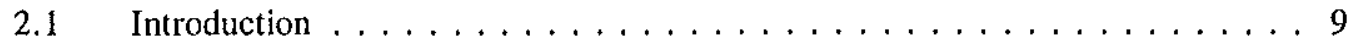

2.2 Methods of economic evaluation $\ldots \ldots \ldots \ldots \ldots \ldots \ldots \ldots \ldots$

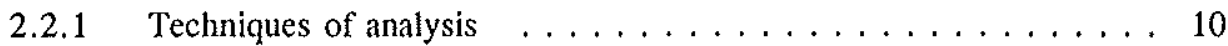

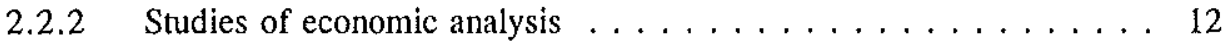

2.2 .3 Areas of debate $\ldots \ldots \ldots \ldots \ldots \ldots \ldots \ldots \ldots \ldots \ldots$

2.3 Measuring and evaluating outcome $\ldots \ldots \ldots \ldots \ldots \ldots \ldots$

2.3.1 Data from current randomised clinical trials . . . . . . . . 16

2.3.2 Measuring and valuing quality of life $\ldots \ldots \ldots \ldots \ldots 18$

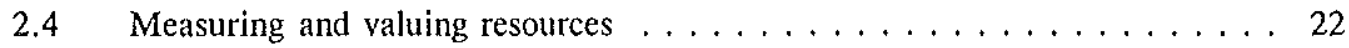

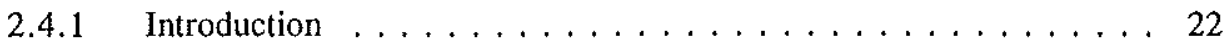

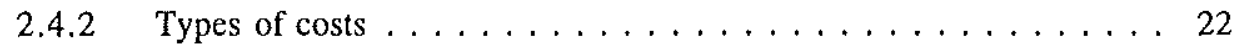

2.4 .3 Perspective and scope of costing $\ldots \ldots \ldots \ldots \ldots \ldots \ldots \ldots$

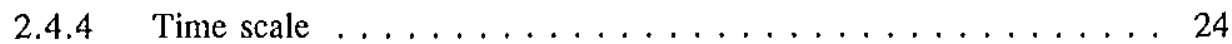

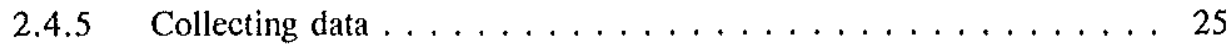

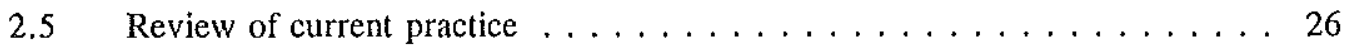

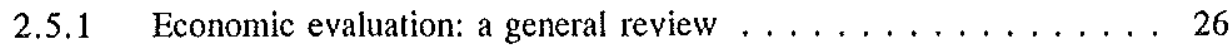

2.5.2 Review of studies specific to cancer $\ldots \ldots \ldots \ldots \ldots \ldots$ 
2.6 Use of results of economic evalations $\ldots \ldots \ldots \ldots \ldots \ldots \ldots \ldots$

2.6 .1 Use by policy makers $\ldots \ldots \ldots \ldots \ldots \ldots \ldots \ldots \ldots \ldots$

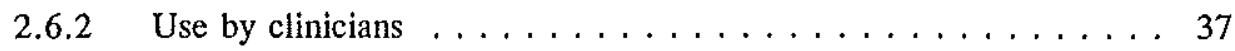

2.6 .3 Use of the results of economic evaluation $\ldots \ldots \ldots \ldots \ldots \ldots$

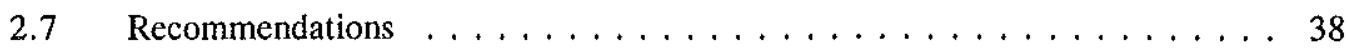

3. Economic evaluation alongside cancer trials $\ldots \ldots \ldots \ldots \ldots \ldots$

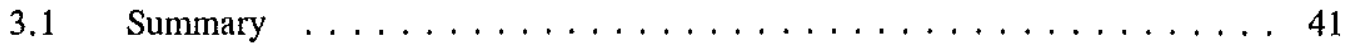

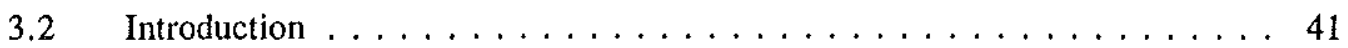

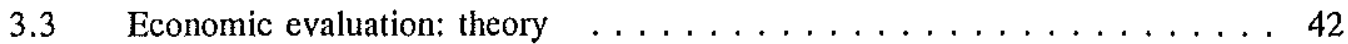

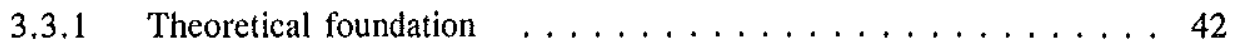

3.3 .2 Choice of perspective $\ldots \ldots \ldots \ldots \ldots \ldots \ldots \ldots \ldots \ldots$

3.3.3 Choice of approach $\ldots \ldots \ldots \ldots \ldots \ldots \ldots \ldots \ldots$

3.4 Economic evaluation as an add-on to a controlled clinical trial . . . . . 44

3.4 .1 Mode of comparison $\ldots \ldots \ldots \ldots \ldots \ldots \ldots 4$

3.4 .2 Choice of alternatives $\ldots \ldots \ldots \ldots \ldots \ldots \ldots \ldots \ldots$

3.4.3 Choice of end points and follow-up time $\ldots \ldots \ldots \ldots \ldots \ldots 45$

3.4 .4 Determination of outcome $\ldots \ldots \ldots \ldots \ldots \ldots \ldots$

3.4.5 Determination of costs $\ldots \ldots \ldots \ldots \ldots \ldots \ldots \ldots \ldots$

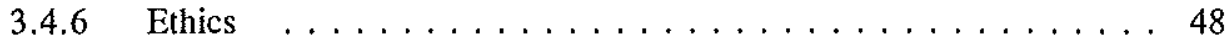

3.4 .7 Generalisation $\ldots \ldots \ldots \ldots \ldots \ldots \ldots \ldots \ldots \ldots \ldots \ldots$

3.5 Specific issues in economic evaluation in cancer . . . . . . . . . . . 49

3.6 Discussion . . . . . . . . . . . . . . . . . . . 50

4. Measurement and valuation of quality of life $\ldots \ldots \ldots \ldots 1$

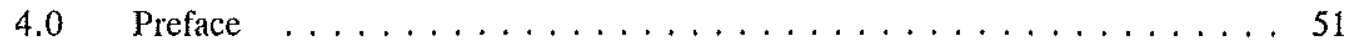

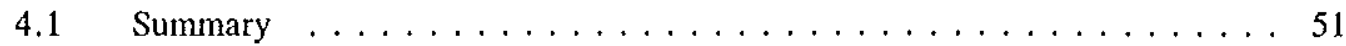

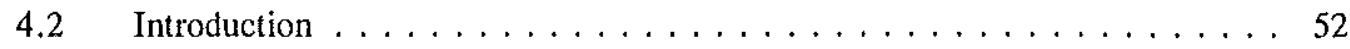

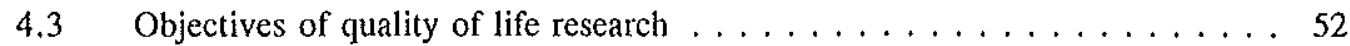

4.4 Description and valuation of quality of life in economic evaluation . . . . . 54

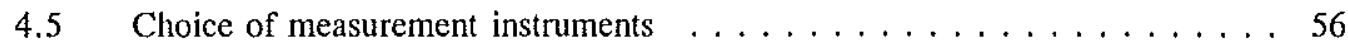

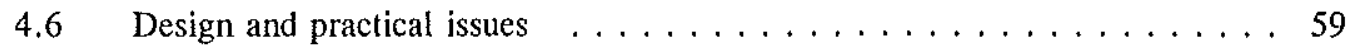

4.7 Discussion . . . . . . . . . . . . . . . . . . 61 


\section{Part II Cost of stem cell transplantations}

5. ABMT in non-Hodgkin's lymphoma and acute leukaemia $\ldots \ldots \ldots 5$

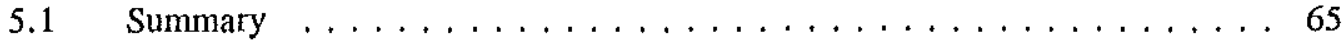

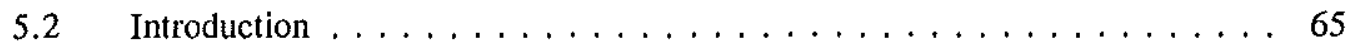

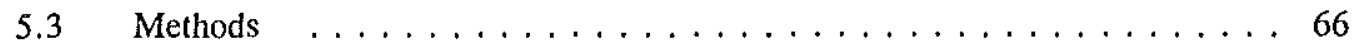

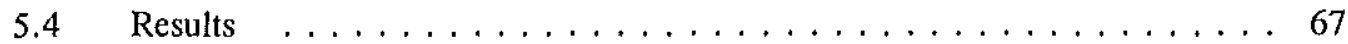

5.4 .1 Results non-Hodgkin's lymphoma group $\ldots \ldots \ldots \ldots 7$

5.4 .2 Results acute leukaemia group . . . . . . . . . . . . . . . . 69

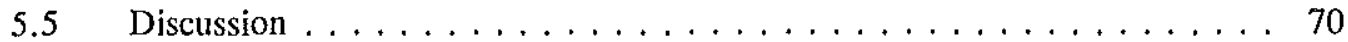

6. Stem cell transplantations in several patient groups $\ldots \ldots \ldots \ldots \ldots$

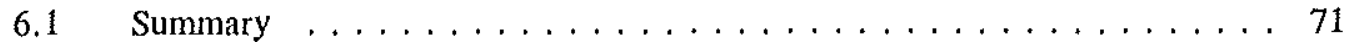

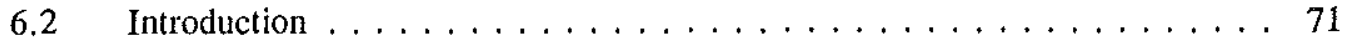

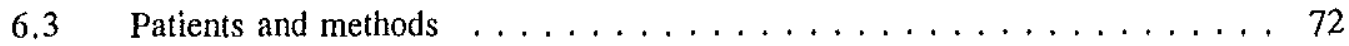

6.4 Unit prices $\ldots \ldots \ldots \ldots \ldots \ldots \ldots \ldots \ldots \ldots$

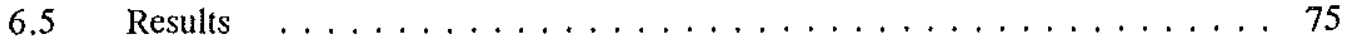

6.5 .1 Clinical results $\ldots \ldots \ldots \ldots \ldots \ldots$

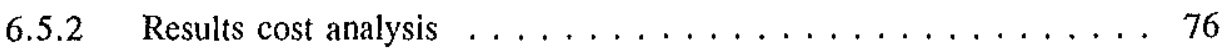

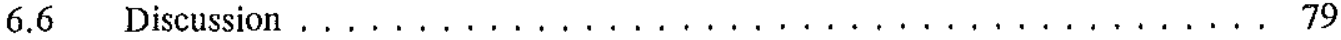

7. Stem cell transplantation in patients with multiple myeloma $\ldots \ldots \ldots$. . . 81

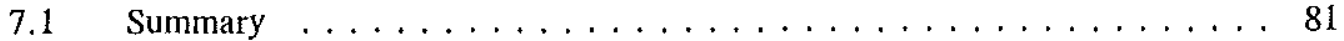

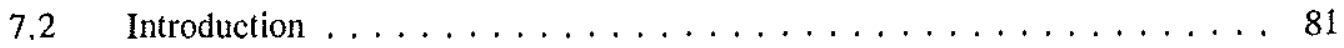

7.3 Patients and methods $\ldots \ldots \ldots \ldots \ldots$

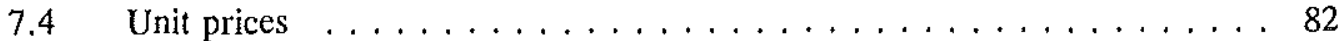

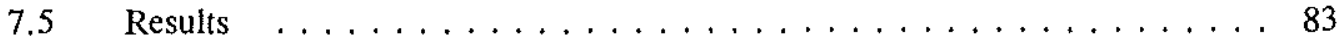

$7.5 .1 \quad$ Clinical results $\ldots \ldots \ldots \ldots \ldots$

7.5 .2 Results cost analysis . . . . . . . . . . . . . . . 84

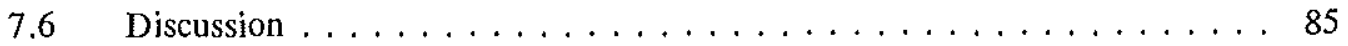

Pant III Prospective economic appraisals

8. ABMT in non-Hodgkin's lymphoma . . . . . . . . . . . . . . 89

8.1 Summary . . . . . . . . . . . . . . . . . . . . 89

8.2 Introduction $\ldots \ldots \ldots \ldots \ldots \ldots \ldots \ldots$ 


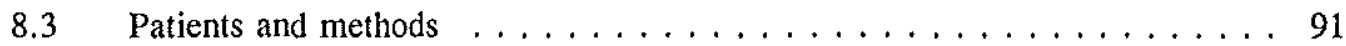

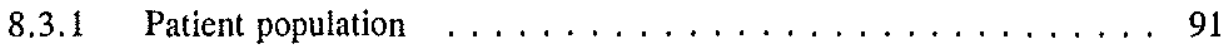

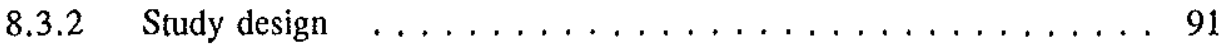

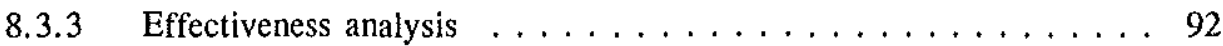

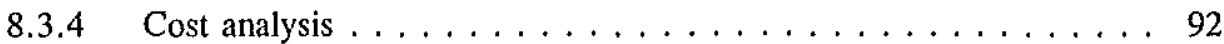

8.3 .5 Markov model $\ldots \ldots \ldots \ldots \ldots \ldots \ldots \ldots \ldots \ldots \ldots$

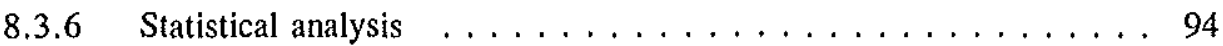

8.4 Results effectiveness analysis $\ldots \ldots \ldots \ldots \ldots$. . . . . . . . . 94

8.4 .1 Treatment response $\ldots \ldots \ldots \ldots \ldots \ldots \ldots \ldots \ldots$

8.4 .2 Overall survival and disease-free survival $\ldots \ldots \ldots \ldots \ldots 95$

8.4 .3 Quality of life study $\ldots \ldots \ldots \ldots \ldots \ldots$

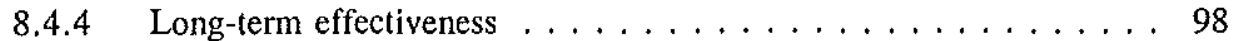

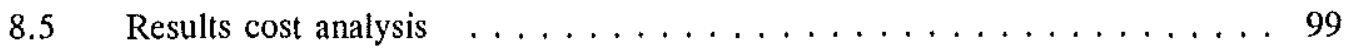

8.5.1 Costs of treatment and follow-up $\ldots \ldots \ldots \ldots \ldots \ldots 9$

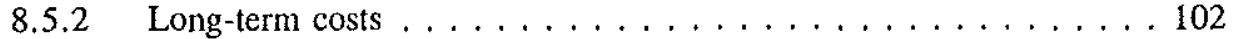

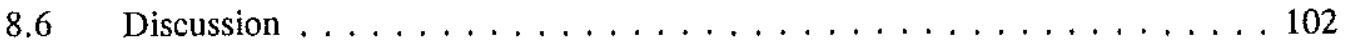

9. GM-CSF in elderly patients with acute myeloid leukaemia $\ldots \ldots \ldots 105$

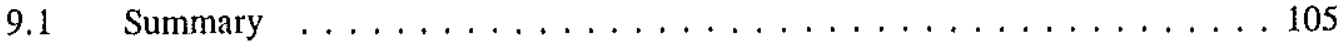

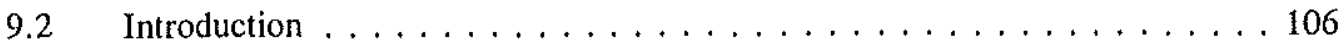

9.3 Patients and methods . . . . . . . . . . . . . . . . 108

9.3 .1 Study design and treatment $\ldots \ldots \ldots \ldots \ldots \ldots \ldots \ldots \ldots$

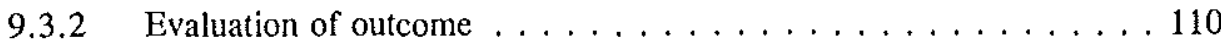

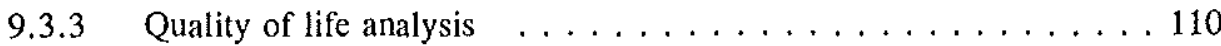

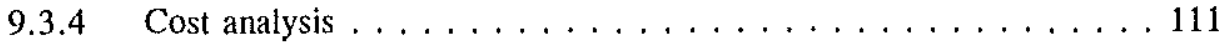

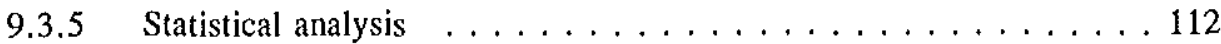

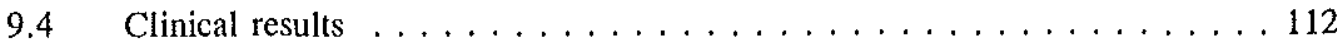

9.4.1 Response to treatment . . . . . . . . . . . . . . 112

9.4 .2 Overall survival and disease-free survival $\ldots \ldots \ldots \ldots \ldots \ldots 114$

9.4.3 Hospitalisation, GM-CSF and haematopoietic recovery . . . . 115

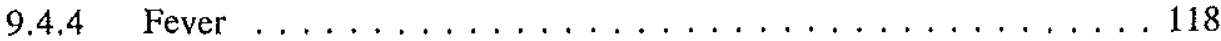

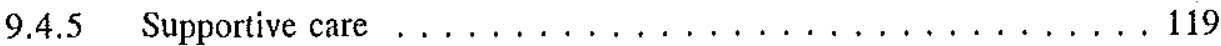

9.4 .6 Toxicity . . . . . . . . . . . . . . . . . . 119

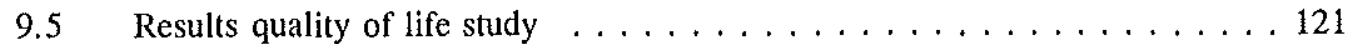

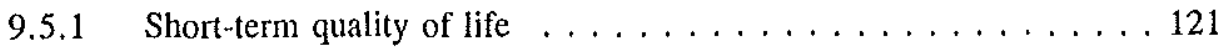

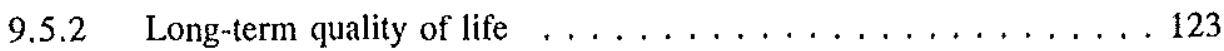

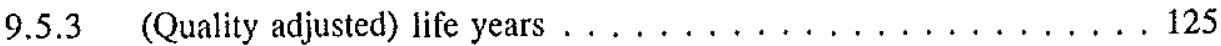




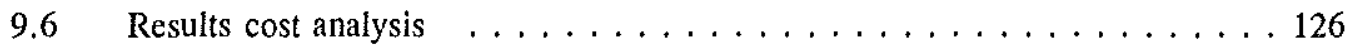

9.6.1 Costs of induction and consolidation treatment . . . . . . . 126

9.6.2 Costs of follow-up and total costs of AML treatment . . . . . . 129

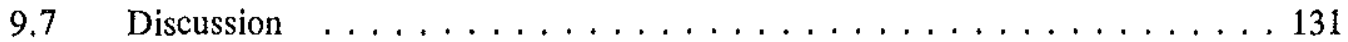

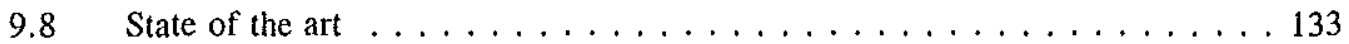

10. GM-CSF in chemotherapy-related febrile neutropenia . . . . . . . . . 137

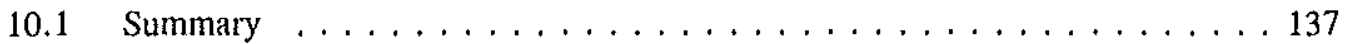

10.2 Introduction . . . . . . . . . . . . . . . . . . . . 138

10.3 Patients and methods . . . . . . . . . . . . . . . . . . . . . 139

10.3 .1 Study population . . . . . . . . . . . . . . . . . 139

10.3.2 Design and treatment . . . . . . . . . . . . . . . 139

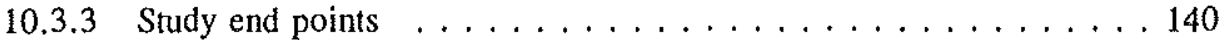

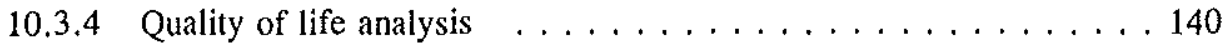

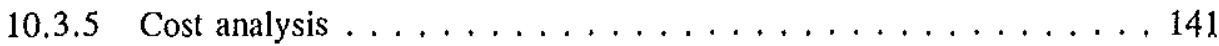

10.3.6 Measurement of serum cytokine levels . . . . . . . . . . . 142

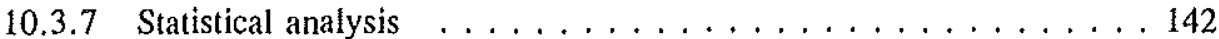

10.4 Results . . . . . . . . . . . . . . . . . . . 143

10.4.1 Patient characteristics . . . . . . . . . . . . . . 143

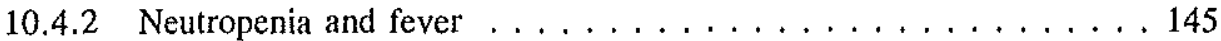

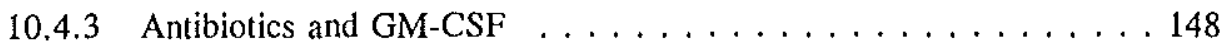

10.4.4 Subgroup, adjusted and multivariate analysis $\ldots \ldots \ldots \ldots \ldots 148$

10.4.5 Supportive care . . . . . . . . . . . . . . . . . . . . . 149

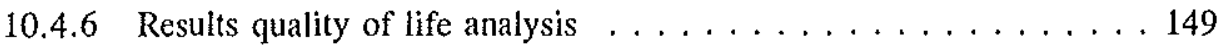

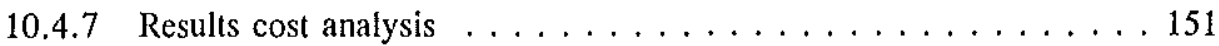

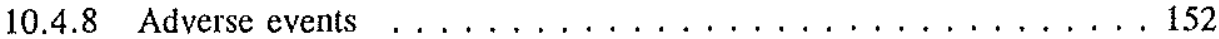

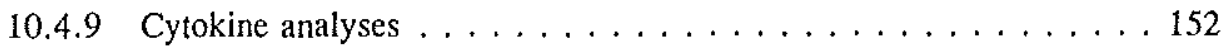

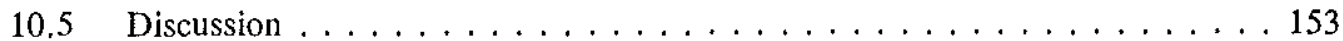

Part IV Implementation of new technologies

11. Costs of introducing ABMT in the Netherlands $\ldots \ldots \ldots 159$

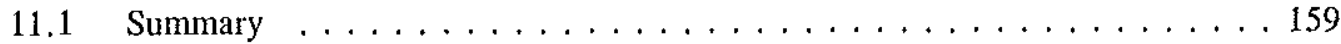

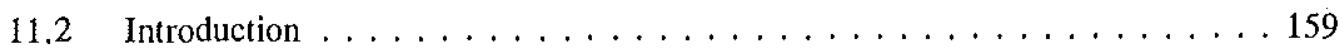

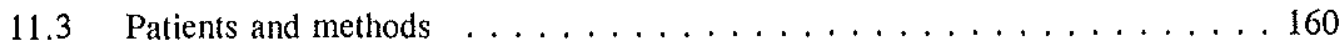

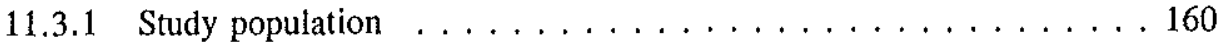

11.3 .2 Treatment modalities $\ldots \ldots \ldots \ldots \ldots \ldots \ldots \ldots \ldots$ 


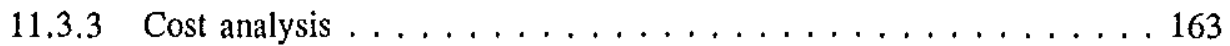

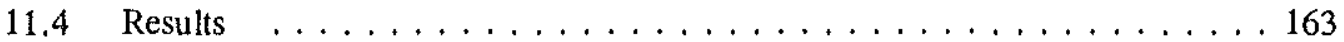

11.4.1 Treatment cost NHL group $\ldots \ldots \ldots \ldots \ldots \ldots \ldots \ldots \ldots$

11.4.2 Treatment cost AML group $\ldots \ldots \ldots \ldots \ldots \ldots \ldots \ldots$

11.4.3 Costs of introducing ABMT $\ldots \ldots \ldots \ldots \ldots \ldots \ldots \ldots$

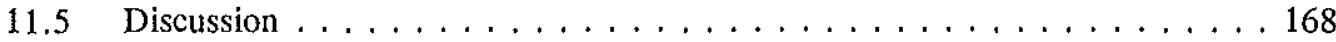

12. Hospital economic model for $\mathrm{HGF}$ therapy $\ldots \ldots \ldots \ldots \ldots 17$

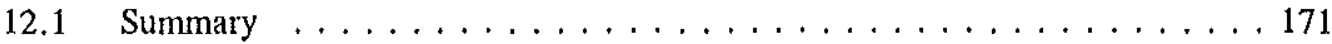

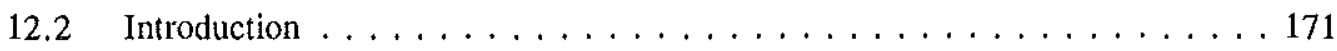

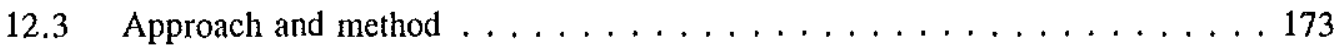

12.3.1 Development of the model $\ldots \ldots \ldots \ldots \ldots \ldots \ldots \ldots \ldots$

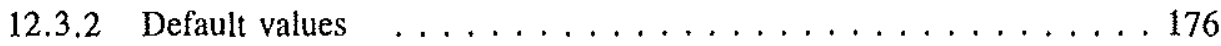

12.3 .3 Unit prices . . . . . . . . . . . . . . . . . 177

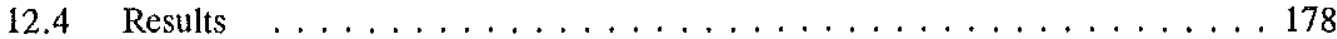

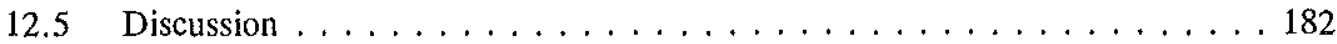

13. Discussion . . . . . . . . . . . . . . . . . . . . 183

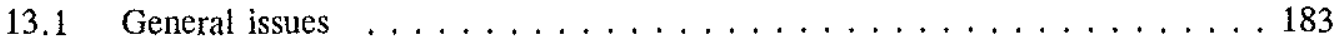

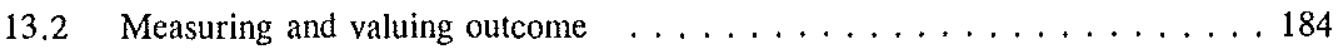

13.3 Measuring and valuing resources $\ldots \ldots \ldots \ldots \ldots \ldots \ldots \ldots \ldots \ldots$

13.4 Generalisability of results $\ldots \ldots \ldots \ldots \ldots \ldots \ldots$

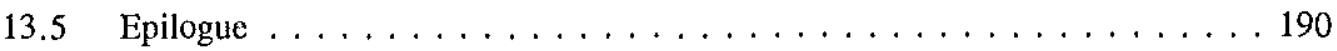

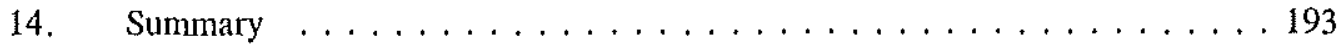

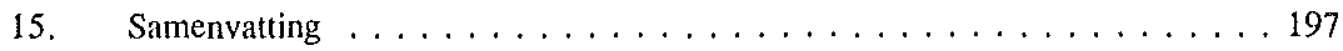

Appendix

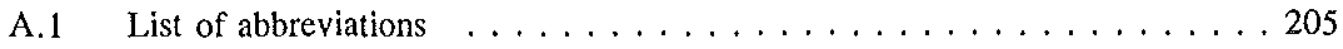

A.2 References . . . . . . . . . . . . . . . . . . . . . . 207

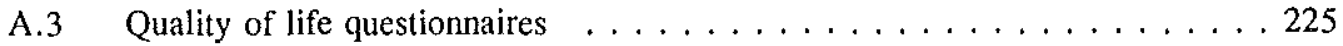

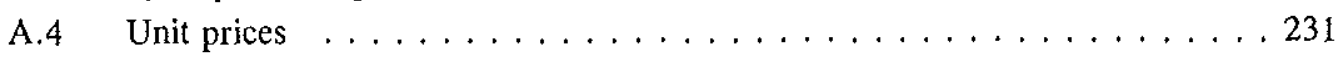

Acknowledgements . . . . . . . . . . . . . . . . . 233 


\section{Publications}

The chapters $2-12$ are based on the following papers:

Chapter 2. Williams C, Coyle D, Gray A, Hutton J, Jefferson T, Karlsson G, Kesteloot K, Uyl-de Groot CA and Wait S. Cost-effectiveness in cancer care. Report of a Working Party from the European School of Oncology. Eur J Cancer (accepted).

Chapter 3. Bonsel GJ, Rutten FFH and Uyl-de Groot CA. Economic evaluation alongside cancer trials; methodological and practical aspects. Eur $J$ Cancer 1993; 29A: S10-S14.

Chapter 4. Uyl-de Groot CA, Rutten FFH and Bonsel GJ. Measurement and valuation of quality of life in economic appraisal of cancer treatment. Eur $J$ Cancer 1994; 30A: 111-117.

Chapter 5. Uyl-de Groot CA and Rutten FFH. The costs of autologous bone marrow transplantation for non Hodgkin's lymphoma and acute leukaemia. Ned Tijdschr Geneeskd 1994; 138: 28-31 (in Dutch).

Chapter 6. Uyl-de Groot CA, Richel DJ and Rutten FFH. Peripheral blood progenitor cell transplantation mobilised by G-CSF (filgrastim); a less costly alternative to autologous bone marrow transplantation. Eur $J$ Cancer 1994; 30A: 1631-1635.

Chapter 7. Uyl-de Groot CA, Ossenkoppele GJ, Riet AAPM van der, and Rutten FFH. Cost analysis of peripheral blood progenitor cell reinfusion mobilised by G-CSF (filgrastim) following high-dose Melphalan as compared with high-dose Melphalan therapy in multiple myeloma. Eur $J$ Cancer 1994; 30A: 457-459.

Chapter 8. Uyl-de Groot CA, Hagenbeek A, Verdonck LF, Löwenberg B and Rutten FFH. Cost-effectiveness of autologous bone marrow transplantation in comparison with CHOP chemotherapy in patients with intermediate- and high-grade non-Hodgkin's Lymphoma (NHL). Bone Marrow Transplant (in press). 
Chapter 9. Löwenberg B, Uyl-de Groot CA, Vellenga E, Willemze R, Suciu S and Rutten FFH. Cost-effectiveness of GM-CSF as an adjunct to intensive remission induction chemotherapy in elderly patients with acute myeloid leukaemia. Report to the Dutch Health Executive Board, 1995.

Chapter 10. Vellenga E, Uyl-de Groot CA, Wit R de, Keizer HJ, Löwenberg B, Haaft MA ten, Witte ThIM de, Verhagen CAH, Stoter G, Rutten FFH, Smid WM, Mulder NH and Vries EGF de. Randomised placebo controlled trial of GM-CSF in patients with chemotherapy-related febrile neutropenia (submitted).

Chapter 11. Uyl-de Groot CA, Okhuijsen SY, Hagenbeek A, Huijgens PC, Imhoff GW van, Löwenberg B, Verdonck LF and Rutten FFH. The costs of introducing ABMT in the treatment of lymphoma and acute leukaemia patients in the Netherlands. Bone Marrow Transpl 1995; 15: 605-610.

Chapter 12. Uyl-de Groot CA, Vellenga E and Rutten FFH. An economic model to assess the savings from a clinical application of haematopoietic growth factors (HGF) (submitted). 
Economic evaluation of cancer treatments 



\subsection{Cancer and economic evaluation}

Cancer is an important cause of illness and death, accounting for a high percentage of mortality in Western countries. In the Netherlands, about $30 \%$ of all deaths is due to cancer and the prevalence, an indicator of the present burden of illness to society, is clearly rising (Coebergh, 1991). In the last decades cancer treatment has shown a rapid evolution. It is now a multidisciplinary treatment strategy incorporating surgery, radiotherapy, chemotherapy and immunotherapy. The high incidence and prevalence of cancer make this disease a major economic issue. The direct medical costs are considerable and are still rising due to the increased use of expensive drugs, radiotherapy equipment, the growing attention to various kinds of palliative interventions and survival success. In the Netherlands, the total direct medical costs of malignant cancer amounted to 1052 million dollars in 1988, that is $4.8 \%$ of total health care costs. About $60 \%$ of this expenditure was produced by inpatient hospital care, about $30 \%$ by outpatient hospital care and about $10 \%$ by non-hospital care (Koopmanschap et al., 1994). It is expected that in 2020, as a result of ageing, these costs will have increased much more rapidly than total health care costs. Finally, the high prevalence of morbidity, mortality and the consequent loss in production also cause high indirect costs.

As health care budgets have grown, an increasing emphasis is being placed on identifying and improving value for money. Therefore, efficacy can no longer be the only criterion that determines whether or not a technology should be used in caring for patients in general and cancer patients in specific. This is true at the level of public health policy as well as at the level of clinical practice. For example the Health Insurance Executive Board has concluded that cost-effectiveness should be taken into account when approving insurance coverage for new treatment modalities. Practising oncologists, faced with pressures to control health care costs, are increasingly being asked to incorporate 
considerations of cost into their decisions regarding the care they provide to their patients. Note that although economic evaluations could provide important information to decisionmakers, it addresses only one dimension of health care programme decisions. Economic evaluation is most useful and appropriate when preceded by evaluation of the efficacy and effectiveness (Drummond, 1987I).

The first part of this thesis deals with methodological and practical issues of economic evaluation in general and in cancer treatment in specific. As cancer treatment may have important side effects, not only life years gained, but also the quality of the life years gained are at issue. Because of this trade-off between the adverse effects of treatment against the adverse effects of the disease itself, quality of life measurement deserves special attention in economic evaluations of cancer treatment.

\subsection{Cancer and new treatment modalities}

During the last few decades, patients with cancer can benefit from new therapeutic approaches, including autologous bone marrow transplantation (ABMT), the administration of haematopoietic growth factors (HGFs) and peripheral blood progenitor cell (PBPC) transplantation. Stem cell transplantation (SCT), both ABMT and PBPC transplantation alike, has significantly improved remission rates and survival in the treatment of malignant lymphomas and acute leukaemias. However, the costs of SCT are high and the additional burden that it places on hospital budget raises concerns (Dufoir et al., 1992; Welch and Larson, 1989).

Transplantations with stem cells derived either from bone marrow or from peripheral blood, are performed to hasten neutrophil recovery. PBPC transplantation is increasingly used in the treatment of malignancies to alleviate bone marrow toxicity resulting from high-dose chemotherapy. It is introduced as an alternative to ABMT, in patients with haematological- and oncological malignancies. Although SCT could improve treatment outcome, the treatment associated risk of fatal complications is high and the patients' morbidity can be serious due to pancytopenia. HGFs make it possible to accelerate haematopoietic recovery after an SCT and thereby reduce the therapy-related toxicity. Several HGFs have been cloned, such as granulocyte colony-stimulating factor (G-CSF) and granulocyte-macrophage colony-stimulating factor (GM-CSF). However, patients receiving HGFs still had a median duration of severe neutropenia for two weeks and had more than three weeks of thrombocytopenia (Nemunaitis et al., 1991). 
The second part of this thesis deals with the costs of SCT, including ABMT, ABMT with HGF and PBPC transplantation. The results are based on retrospective studies of detailed patient records. The first cost study focuses on ABMT in the treatment of patients with malignant lymphoma and acute leukaemia. In the Netherlands, little is known about the cost of ABMT. This kind of information is important for the reimbursement policy. This study aims at calculating the average costs of ABMT in the Netherlands. The costs of ABMT in relation to $\mathrm{PBPC}$ transplantation are compared in the second study. The patients received ABMT without the colony stimulating factor G-CSF (filgrastim), ABMT with GCSF or PBPC transplantation mobilised by G-CSF. The patient population consisted of solid tumour patients and patients with malignant lymphomas. The last cost study focuses on the costs associated with the treatment of patients with multiple myeloma. The treatment modalities are high-dose melphalan with and without G-CSF on the one hand and G-CSF mobilised PBPC transplantation following high-dose melphalan on the other hand.

Furthermore, three prospective economic evaluations concerning ABMT and HGFs have been carried out. These studies are cost-effectiveness analyses and include a quality of life measurement. The first study addresses the question whether patients with non-Hodgkin's lymphoma who show only partial response to initial chemotherapy will benefit from early high-dose chemo-radiotherapy and ABMT. The aim of this study is to assess the effectiveness, quality of life and cost implications of autologous bone marrow transplantation (ABMT) versus standard chemotherapy.

The second study concerned the treatment of patients with acute myeloid leukaemia (AML). AML is mainly seen in elderly patients. The generally reduced tolerance of older patients to the toxicity of chemotherapy and the increased preleukaemic conditions may require special treatment strategies for AML in the elderly. Only few studies especially dealt with therapy development of AML in elderly patients. The advent of HGFs which on the one hand may improve the response and on the other shorten hand the neutrophit regeneration after chemotherapy might be especially important in improving the outcome of the older category of AML patients. The aim of this analysis was to compare the costs and effectiveness of GM-CSF (molgramostim) as an adjunct to intensive remission induction chemotherapy. Specific attention has been given to both the short- and longterm quality of life of the patients.

The third study focuses on patients with chemotherapy-related fever and neutropenia. Neutropenia is a frequent and serious complication of cancer chemotherapy. When neutropenia is complicated by fever, infection is usually assumed to be present. So, 
neutropenic fever represents an indication for empiric antibiotic therapy, requiring additional health care resources. HGFs reflect a new and effective means of treating chemotherapy patients to prevent fever and neutropenia. The use of HGFs may reduce the duration and/or severity of neutropenia. Also, the administration of HGFs may lead to significant cost savings, by reducing the stay in hospital, and may improve quality of life.

As resources are limited, the implementation of ABMT and HGFs in clinical practice may have major macro- and microeconomic consequences. Concerning ABMT, the macroeconomic consequences of introducing ABMT in terms of the additional resources required to finance $\mathrm{ABMT}$ for patients with non-Hodgkin's lymphoma (NHL) and AML are studied.

The final paper describes the implementation of HGFs in the daily hospital practice by means of a hospital economic model. The economic model deals with all relevant direct costs and savings in relation to chemotherapy-induced fever and neutropenia. In this model, a distinction is made between patients receiving intensive and standard chemotherapy schedules. The latter is subdivided in prophylactic administrations and administrations in patients with chemotherapy-induced fever and neutropenia. By means of a literature review, default values have been estimated for a number of cases. Furthermore, sensitivity analyses have been carried out and a Markov model is specified.

\subsection{Contents}

Part I deals with methodological and practical issues concerning economic evaluation of cancer treatments. Chapter 2 briefly discusses basic methods of economic evaluation, the way of measuring and valuing effectiveness and resources. Also, the current practice of economic evaluation is described by reviewing screening and lung cancer. Chapter 3 also concerns methodological and practical aspects, but it focuses on economic evaluation alongside cancer trials. Chapter 4 describes how to measure and value quality of life in economic evaluation.

In part II, the results of several costs analyses are presented. Chapter 5 considers the costs of ABMT in lymphoma and acute leukaemia patients. It is the first study concerning the costs of ABMT in the Netherlands. An alternative to ABMT is PBPC transplantation. In Chapter 6, ABMT is compared with PBPC transplantation in patients with haematological as well as oncological malignancies. Furthermore, PBPC transplantation in 
combination with high-dose chemotherapy may be a treatment alternative to high-dose chemotherapy alone. Chapter 7 considers SCT in patients with multiple myeloma.

Part III presents the results of three cost-effectiveness studies. The first study concerns patients with NHL, who had a partial response and NHL free bone marrow after three cycles with conventional chemotherapy (Chapter 8). The patients received either ABMT or CHOP chemotherapy. The other two studies deal with the haematopoietic growth factor GM-CSF. Chapter 9 describes the results of a study comparing the costs and effects of GM-CSF as an adjunct to intensive chemotherapy in elderly patients with AML. Chapter 10 deals with the administration of GM-CSF in patients with chemotherapyrelated fever and neutropenia.

Part IV considers the implementation of ABMT and HGFs in the clinical practice. Chapter 11 describes the costs of introducing ABMTs in the Netherlands from a macroeconomic perspective. In Chapter 12 the cost consequences of introducing HGFs in the daily hospital practice are described by means of a hospital economic model.

Chapter 13 concludes with a discussion and with recommendations for further research. Chapter 14 and 15 summarises the previous parts in English and Dutch, respectively. The appendix presents the meaning of abbreviations, bibliographic references, quality of life questionnaires and unit prices. 


\section{Part II}

Methodological and practical issues 



\section{Cost-effectiveness in cancer care}

\section{$2.1 \quad$ Introduction}

The aim of this chapter is to examine the need for economic evaluation of cancer care and to stress the importance that any such evaluation is carried out in a rigorous and effective manner. As pressures on health care budgets have grown worldwide, an increasing emphasis is being placed on identifying and improving value for money: that is, maximising the health gain from resources committed to health care by concentrating on interventions of proven effectiveness and cost-effectiveness.

To date, the cost-effectiveness literature across all areas of health care remains fairly small, despite strong growth in recent years: between 1960 and 1992 a total of 636 economic evaluations combining cost and outcome data were published, or less than two a month (Backhouse et al., 1992). Another survey identified an annual average of 247 studies containing some form of evaluation between 1979 and 1990 (Elixhauser et al., 1993). The quality of these studies is known to be highly variable. Although more studies have been published with time, this has only kept pace with the increasing number of clinical trials.

This chapter will briefly discuss basic methods of economic evaluation. This will be followed by a section discussing current methods of measuring and valuing the effectiveness of care. Measuring and valuing of resources is covered in the next section. Current practice of economic evaluation is covered in a section which reviews the general situation in medicine and then discusses two areas of cancer care - lung cancer and screening. The penultimate section discusses the use of results of economic evaluations. The final part consists of a series of recommendations applicable to a wide variety of individuals involved in all aspects of cancer care. 


\subsection{Methods of economic evaluation}

This section briefly introduces concepts and techniques used in economic evaluation. For in depth discussion readers are referred to the following references: Department of Health, 1992; Drummond et al., 19871 and 1987II; Luce and Elixhauer, 1990.

\subsubsection{Techniques of analysis}

Economics offers a range of techniques for the evaluation of health care activities. Each has its place, but it is important to ensure that the appropriate method is being used in any particular context.

Cost-minimisation analysis (CMA): The least complicated approach to economic evaluation is to judge between alternatives on the basis of minimum cost. In doing this it is essential to establish a priori that the treatment options being compared are identical in their effectiveness and risk of complications and side-effects, i.e. that resource cost is the only significant difference between the options. Good examples of such studies can be found in the literature (e.g. Lowson et al., 1981) on long-term domiciliary oxygen therapy, but the approach is likely to have limited applicability in the cancer field because of the variable impact of treatments on patient survival and quality of life.

Nevertheless, this approach can be useful for comparing different treatment techniques with similar patient outcome. In radiotherapy treatment for instance, one can choose between off-line or on-line in vivo dosimetry and portal imaging techniques. For each of them, a choice can be made on the basis of cost information (Kesteloot et al., $1993 \mathrm{I}$ and 1993II), since the patient outcomes are very similar with both techniques. For another quality assurance technique, the use of fixation masks, no difference in patient outcome (i.e. reproducibility of the patient set-up) could be detected, implying that the choice between PVC (plastic) and thermoplastic (Orfit) masks can be based on cost data (Weltens et al., 1993).

Cost-effectiveness analysis (CEA): This is the commonest approach to economic evaluation in health care, enabling the comparison of alternatives with both differing cost and differing effectiveness: the latter being measured in natural units such as life years saved (e.g. Ludbrooke (1981) compared treatments for renal failure in this way). New medical treatments may be more effective and are often more costly and their impact can be 
clearly expressed using the cost-effectiveness ratio, i.e. the extra cost of each extra life year gained by switching to the new treatment.

$$
\Delta \mathrm{CE}=\frac{\mathrm{C}_{1}-\mathrm{C}_{2}}{\mathrm{E}_{1}-\mathrm{E}_{2}} \quad \begin{aligned}
& \text { where } 1 \text { is the new } \\
& \text { treatment and } 2 \text { is } \\
& \text { the current treatment }
\end{aligned}
$$

Cost-effectiveness is a relative concept, the ratio must always be calculated in the context of a comparison with existing or potentially competing treatments. Thus, new treatments for teratoma will concentrate on potential further improvement in survival over existing chemotherapy, whereas in advanced non-small cell lung cancer improved palliation will be sought.

Cost-utility analysis (CUA): The limitation of CEA is that it requires a single measure of effectiveness by which to compare procedures. Often treatments will have a range of outcomes and these outcomes will be valued differently by different patient groups. Recent developments in economic evaluation have attempted to measure multi-dimensional outcomes and to incorporate preferences in the assessment of the effectiveness of health care. Thus, an attempt is made to measure the effect of health care on the lives of patients (in economic terms its utility to them), rather than just its impact on clinical variables. For example, to judge the success of chemotherapy in terms of tumour control may miss the aspects of the treatment which have most impact on patients. The most common utility measure used is the quality adjusted life year (QALY), which combines the benefits of survival and quality of life during the survival period. The results of CUA are also presented in ratio form as the cost per QALY gained from the adoption or expansion of a health care programme. As well as being a better measure of effectiveness, CUA also enables comparisons to be made between programmes for different patient groups as the utility measures are not disease specific.

Cost-benefit analysis (CBA): The three approaches to economic evaluation described above are designed to answer specific questions regarding the choice between competing health care interventions when a decision has already been made to do something. CEA and CUA can therefore be useful in allocating limited budgets between competing services. They do not address the more fundamental question of whether some health care activities are worth doing at all. The economic technique designed to deal with such questions is CBA. This differs from CEA and CUA in that it calculates the benefits in monetary terms as well as the costs. Thus, if some of the benefits are not directly purchased by people as consumers (as in the majority of health care), then proxy 
measures of monetary benefits must be calculated. The danger with using this approach is that items which are too difficult or too controversial to apply monetary values may be ignored, although they are important. The advantage of CBA is that it provides a measure of the rate of return to investment in health care which can be compared with the rates of return from other public and also private investments.

Early CBA studies in health care were often limited to those factors most easily quantified and used poor proxies for the value of improved survival and length of life. More recently renewed interest in $\mathrm{CBA}$ in health care has been generated by the successful use of the technique in other areas such as environmental economics. The monetary value of health benefits can be estimated by measuring the patients' willingness-to-pay through questionnaire surveys (Johanssen and Jonsson, 1991). This approach is the subject of much debate amongst economists and is unlikely to become routinely applied in the near future (Hutton, 1992).

\subsubsection{Studies of economic analysis}

Whatever the method of economic evaluation chosen, the analysis must follow the same framework. The basic stages are:
a. problem definition;
b. identification of options;
c. identification of relevant costs and outcomes;
d. measurement of costs and effectiveness of each option;
e. allowance for differential timing of costs and benefits;
f. assessment of risk and uncertainty;
g. presentation of results.

a. Problem definition: This is a crucial stage as it dictates the subsequent conduct of the analysis. A key factor is to determine the perspective from which the analysis is being conducted. This governs which costs and outcomes are considered. For example, if the perspective of the health service is taken then costs falling on other government agencies, such as social services, and patients may be ignored. An analysis carried out from the perspective of society as a whole would include these costs. The scope of the problem will determine the economic evaluation technique to be used. Thus, selecting the most appropriate imaging technique to make a given diagnosis may require CEA and the choice between chemotherapy or surgery in the treatment of cancer requires CUA. 
b. Identification of options: An economic study will only be valid if comparison is made between a new procedure and the best available alternative. In the case of new therapies where no effective treatment currently exists, the assessment of costs and outcomes must be made against current patterns of care, however ineffective.

c. Identification of costs and outcomes: The relevant costs and outcome measures will be influenced by the study perspective, the economic method and the health care procedure under analysis.

d. Measurement of costs and effectiveness: Good evidence of clinical effectiveness (or lack of it) is essential for stages of economic analysis. This should come from a controlled clinical trial if possible. Prospectively collected cost data is also preferable, but caution should be taken in using cost data from clinical trials, as the care given is dictated by the trial protocol and may not match subsequent care delivered in routine situations.

e. Allowance for differential timing of costs and outcomes: When comparing alternative health care programmes the pattern of costs and benefits may differ significantly between options under review. For example, health promotion and preventive health care programmes incur immediate costs, but the main health benefits will only be realised many years into the future. Acute surgical treatment has a high immediate cost, but, if successful, gives immediate benefits to the patient. Long-term maintenance drug therapy (e.g. for hypertension or peptic ulcer) may have a relatively low annual cost, but builds-up to a substantial amount over a lifetime. Simply adding total costs and benefits over time is not adequate because people have distinct preferences for delaying costs and bringing forward benefits, the flow through time matters as well as the absolute size of costs and benefits. To take into account this "time preference" in the economists' parlance, discounting of costs and benefits is necessary. In essence this process reflects the declining importance in individuals' decision making of outcomes occurring further and further into the future, by reducing their value (for analytical purposes) by a given percentage for each year ahead they are expected to occur. This produces estimates of costs and benefits valued at a single point in time, i.e. their present value. Thus, most decisions in lung cancer will not be discounted since their outcome occurs rapidly. In breast cancer discounting may be necessary for preventative or screening strategies, whilst it becomes less necessary the more advanced the disease.

In the context of cancer the importance of the timing of benefit flows would be seen in an evaluation of strategies to reduce the loss of quantity and quality of life from, for example, lung cancer. Health promotion activities to reduce smoking by young people 
could have a major impact, but the benefits would not be seen for many years. On the other hand marginal improvements in the effectiveness of radiotherapy could give small but immediate benefits. The complexity of such analyses is further complicated, since the data can be looked at from the standpoint of society as a whole or from that of the patient.

f. Assessment of risk and uncertainty: In the absence of firm data it is often necessary to make assumptions in estimating the costs of health care. Clinical effectiveness data may also be subject to uncertainty. This must be explicitly recognised and tested using sensitivity analysis. This will identify the key variables and assumptions, which, if changed will alter the conclusions of the study.

g. Presentation of results: This should be done in a way which clearly shows whether the original problem defined has been solved. Where uncertainty has been shown to be present, ranges rather than point estimates should be presented. The results should be placed in their context, indicating the possibility of generalisation to other settings. For those studies designed to help health service decision makers to allocate limited budgets the marginal costs and benefits should in particular be shown.

\subsubsection{Areas of debate}

The above brief review of methods of economic evaluation and the framework within which such evaluations should be conducted is designed to give the reader an overall perspective on the process. Subsequent sections will discuss the development of methods in more detail. Needless to say while the above broad framework is generally accepted by economists there is intense debate on many specific points.

For example, there are many different approaches to the measurement of quality of life and the utilities to be attached to different health states. This makes the interpretation of cost per QALY results very difficult (Gerard, 1992). Although economists lay great emphasis on the need for strong evidence of clinical effectiveness (though effectiveness may differ in the community setting) from randomised controlled trials they are ambivalent about the desirability of collecting economic data in the same way (Drummond and Davies, 1991). Some economists also question the appropriateness of discounting health benefits (as opposed to costs) as time preference may not apply to non-monetary costs and benefits. Amongst those who agree that discounting of benefits should take place there is 
debate as to whether the current Treasury recommended rate of $6 \%$ is appropriate (Parsonage and Neuberger, 1992; Cairns, 1992).

On the cost side the general view is that the main focus should be on direct costs of health care, rather than the indirect costs of illness to society through the loss of working days. This remains a much debated area and recent work has shown that even if lost working time is to be included as a cost, the value placed on it should be much lower than that used in earlier studies (Koopmanschap and Van Ineveld, 1992).

Despite the healthy debate on these and other issues, methods of economic evaluation are sufficiently well established to provide an essential contribution to the debate on the development of health care services. This applies equally to cancer care as to other areas.

Checklist:

Problem definition: Clear definition of problem, statement of perspective of analysis and analytical technique.

Options: There must be a comparison of at least two options (including the status quo).

Identification of costs and outcomes: All costs and outcomes relevant to the study perspective must be identified.

Measurement of costs and outcomes: The best possible source of cost and outcome data should be used e.g. randomised controlled trials may provide this though it is important to note that they may vary from the costs in the community.

Differential timing of cost and benefit flows: Future costs and outcomes must be discounted at a rate appropriate to the study perspective.

Uncertainty: The impact of this should be demonstrated through the use of sensitivity analysis, which analyses the impact of different cost and/or outcome estimates on the final results.

Presentation of results: These should show the marginal impact on cost and effectiveness of each option. 
The above checklist serves as a guide to the key points for those assessing the quality of a completed evaluation or for those planning a new study.

\subsection{Measuring and evaluating outcome}

Although this section, for brevity, concerns itself solely with randomised clinical trials (RCTs), evidence of treatment outcome is often derived from other data. Whilst RCTs remain the "gold standard", where data from such trials is not available, useful information can be gleaned from non RCT sources. The same problems, noted below, of RCTs, of course, still apply and are sometimes amplified. It should be emphasised that, whenever possible, expensive new therapies should be assessed in RCTs large enough to provide a clear result.

\subsubsection{Data from current RCTs}

RCTs have become the "gold standard" by which medical care is evaluated (Sacks et al.; 1982). Despite many thousands of RCTs, many questions remain unanswered and apparently open. Even more damaging may have been the failure of the medical community to identify therapies that do work and acceptance of treatments of little or no efficacy. Systematic reviews and consensus meetings based on such data will help to overcome the problem of RCTs which are too small to give a reliable answer.

The basic reason for our failure to come up with clear answers to questions has been the small size of most RCTs. For example, an ongoing systematic review of all chemotherapy trials including a no chemotherapy control (published and unpublished) in non-small cell lung cancer has only found 70 such RCTs. These include about 10000 patients in all (non-small cell lung cancer overview Collaborators' conference, Cambridge, 24-26 September 1993). Some trials had more than two arms, so that the mean number of patients per arm is about 100 . During the time period of these trials several million people died of lung cancer - less than $1 \%$ of patients with non-small cell lung cancer are entered into chemotherapy trials.

It has taken cancer clinicians a long time to realise that large therapeutic gains are unlikely to be achieved by current therapies. Only now are large scale trials being contemplated. An alternative, currently available, is the tool of systematic review (overview or meta analysis). Such a systematic review may be based on an exhaustive 
analysis of individual patient data from all published and unpublished RCTs. This approach is likely to be more accurate than such an exercise simply carried out on published data. Publication bias, patient exclusion, length of follow-up and rigour of the original analysis are all potential sources of error in a systematic review of published trials. Preliminary data from Stewart and Parmar (1993) suggests that such reviews may overestimate treatment effects when compared to individual patient data overviews.

The utility of systematic review of individual patient data has been clearly demonstrated. Lau and his colleagues (1992) examined all of the RCTs for the therapy of myocardial infarction. These were looked at in a cumulative fashion, so that the evidence (or lack of it) for each treatment could be examined over time. As an example, they found 33 RCTs (36974 patients) of intravenous streptokinase carried out between 1959 and 1988. When examined in a cumulative fashion there was initial evidence of a favourable treatment effect after 8 trials $(1973,2432$ patients, $p=0.007)$ even though the majority of individual trials were negative. By 1977 the p-value was less than 0.001 (14 trials, 4314 patients). Despite these clinical trials continued to be published up to 1988, large scale trials (such as ISIS) are being demanded before clinicians would accept this approach. Similarly, the recent individual systematic review of early breast cancer management has shown unexpected survival benefit for a therapy discarded two decades ago - oophorectomy in premenopausal women (Early Breast Cancer Trialists' Collaborative Group, 1992).

As well as providing data to answer questions, systematic reviews often show up the need for new large scale trials and it is to be hoped that genuinely large trials that provide answers and data with tight confidence intervals will be carried out. Systematic reviews are likely to be seen increasingly often and the Cochrane Collaboration will act as a coordinator stimulating, supporting and making data available from systematic reviews (Herxheimer, 1993). The Cochrane Collaboration is an international network which will provide databases of all past and ongoing RCTs as well as past and present systematic reviews. They will provide, on line, updated systematic reviews of individual questions. The activities will encompass the whole of medicine - a truly monumental task.

When carrying out an economic evaluation it is essential that there is reliable data on efficacy. In the absence of a large definitive trial (the usual case) selection of specific trials or a cursory examination of the literature is likely to lead to a biased result. The difficulty of obtaining reliable data and avoiding bias is demonstrated by the finding that computerised searches of databases like MEDLINE only identify $50 \%$ of published RCTs found by hand searching the literature (Dickenson et al., 1985). 
Additional problems with the current literature include the use of inappropriate controls (for instance trials of chemotherapy in non-small cell lung cancer comparing chemotherapy regimes when the benefit of chemotherapy was unproved) and the failure to measure appropriate end points. Although survival is highly important, concentration on this has meant that there is little or no data on the outcome of therapy on symptom control or quality of life - even when treatment is entirely palliative.

\subsubsection{Measuring and valuing quality of life}

Cancer is an important cause of illness and accounts for a high percentage of crude mortality in Western countries. In the last decades cancer treatment has developed rapidly. It is now multidisciplinary, incorporating surgery, radiotherapy, chemotherapy and/or immunotherapy. Treatment may have major side effects, particularly radio- and chemotherapy. Consequently, patients and those involved in their care have wondered whether improved probability of survival, if it exists, outweigh the burden of these severe side effects in all cases. Therefore, not only life years gained, but also the quality of those years is an important issue.

The usual objective of carrying out quality of life research is to gain insight into the reaction of patients to cancer and its treatment and the interrelations of these different reactions with the overall quality of life. Furthermore, data concerning the impact of a certain therapy on the quality of life may increase knowledge about the effectiveness of such therapy (De Haes and Knippenberg, 1985).

Quality of life studies generally give a description of the health status. Depending on the type of study, they deal with more or less objective features of health. Most studies incorporate the physical and psychological aspects of life, sometimes complemented by the aspect of social functioning. From the economic perspective the objective of studying quality of life is to determine the contribution of changes in quality of life to a summary measure for the outcome of an intervention. When there is more than one effect parameter (e.g. survival and health status improvement) or when outcome is measured in more dimensions of quality of life, a value judgement (e.g. utility weight) should be given to each of these parameters or quality of life dimensions in order to arrive at such a summary measure. This makes it possible to make comparisons across different technologies and across different disease categories. The importance of different outcome parameters will depend on the disease type. Quality of life assumes greater importance in 
advanced breast cancer, whereas survival is of paramount importance in malignant teratoma.

Measurement instruments for description and valuation of quality of life: Descriptive instruments can be divided into two main categories: the generic instruments and diseasespecific questionnaires (Patrick and Deyo, 1989). Generic instruments are developed to measure all health dimensions. They can be used over a wide range of disease categories and are therefore suited to function as generally applicable descriptive instruments for measuring quality of life improvement in economic evaluation. In this respect they provide a reference for quantitative assessment of quality of life changes in economic evaluation and have been used in many economic appraisals. Generic instruments most frequently used are: the Nottingham Health Profile (NHP), the Sickness Impact Profile (SIP), the abbreviated RAND-Medical Outcome Study questionnaire (RAND-MOS 20) and the newly developed Short Form-36 (SF-36) (Uyl-de Groot et al., 1994III).

Disease-specific instruments measure health problems specific for an illness or disease category. These instruments measure specific quality of life aspects typical of a particular disease, and are therefore more sensitive to changes in the health states of patients with that disease. Using cancer as an example, these instruments can be divided into cancer specific questionnaires (for all cancers) and specific cancer questionnaires (for one type of cancer). The following cancer specific instruments are often used: the Functional Living Index for Cancer (FLIC), Spitzer QL index, European Organisation for Research and Treatment of Cancer Core Quality of Life Questionnaire (EORTC QLQ-C30), Cancer Rehabilitation Evaluation System-Short Form (CARES-SF) and the Rotterdam Symptom Checklist (RSCL). Examples of specific cancer instruments are: the breast Cancer Chemotherapy Questionnaire, the Performance Parameters for head and neck cancer and the Linear Analog Self-Assessment (LASA) of Voice Quality for laryngeal cancer (Uyl-de Groot et al., 1994III).

For the description of quality of life in economic evaluation, generic instruments suffice for overall comparison and testing, but the inclusion of specific instruments may be advisable, particularly for reasons of explanation if changes in dimensions are small or conflicting. By using generic instruments it is not always possible to gain insight into how changes in health have taken place.

When strategies score differently on different dimensions and when one needs a summary measure of outcome comparable across a range of interventions, it is necessary to get valuation of health states in one single dimension representing an overall judgement of 
quality of life of a health state. This then would allow the calculation of "quality adjusted life years" (QALYs), where a life year gained is "adjusted" using this value judgement for quality of life. To acquire valuations, respondents are requested to value health states. These states are derived from a set of health state descriptors. The values are usually ranging from 0 to $1(0=$ "worst imaginable health state", and $1=$ "best imaginable health state"). By means of modelling techniques a multi attribute utility function may be derived to predict values of any composition of health states. Methods of acquiring valuations are: direct rating (e.g. determining a point on a line with clearly defined endpoints), standard gamble (choosing between options under uncertainty) and time trade-off (choosing between options with different durations of a particular health status) (Drummond et al., 1989; Torrance, 1987).

Furthermore, there are different basic approaches to measuring the utility of health status. One approach is to find people with the particular health state and solicit their valuation of such health states in terms of a utility. Using such subjective utility assessments is most in line with welfare theory. Another approach is to describe the condition, usually by an abbreviated written scenario, to people who do not have the condition and ask them to make the appropriate valuation (Drummond $e t$ al., 1989). These people are thought to represent public opinion. Other approaches include for instance the patients family or health professionals opinions.

Which approach is preferable, depends on the viewpoint of the study. When a study is conducted from the societal viewpoint and is pertinent to public policy decision making, the appropriate utilities are those of the general public. If a fixed set of utility values is available and if a generic instrument is used to measure health outcomes in an economic evaluation, this adds to the comparability of results from economic evaluations and fits with the public sector context of decision making supported by results from an economic evaluation. The problem with soliciting value judgements from persons without relevant disease experience is that the person should truly understand what the described health state is like. For some of the generic health measurement instruments (e.g. the EuroQol) such a set of utility values is known allowing the calculation of QALYs gained when such a generic instrument is used to assess health outcomes. Available valuation instruments are EuroQol, the Quality of Well-being Scale (QWB-Scale), the McMaster Utility Measurement Questionnaire (MUMQ), the Torrance's Health State Classification System and the Rosser \& Kind-index (Uyl-de Groot et al., 1994III). 
Choice of measurement instruments: In clinical trials the choice of measurement instrument depends on criteria such as psychometric features (internal consistency, reliability, validity, etc.), specific patient features, feasibility and on the possibility of gaining insight into the quality of life aspects of the disease itself and into the side-effects of treatment. Quality of life measurement in economic evaluation should allow comparison across different technologies and diseases. This requires the inclusion of a generic, a cancer specific measurement instrument and a valuation instrument. The precise combination of instruments depends on the required psychometric and specific patient features and on feasibility. (See Chapter 4, Table 4.1 for an overview).

Interview schedule and follow-up time: Ideally the data collected should describe quality of life of patients before, during and after treatment. The choice of time scale depends on the natural history of the disease and on the timing and pattern of treatment. During intensive treatment periods it may be important to have frequent measurement of quality of life, occasionally intervals of 1 or 2 days may be desirable (Fayers and Jones, 1983). If there is a survival benefit it is important to take into account quality of life during life years gained. In this respect, it is necessary to make a distinction between curative and palliative treatment modalities. In both modalities quality of life measurement is very important, but the outcome indicators differ. In palliative treatment modalities, quality of life is the single most important outcome indicator. For curative treatment modalities the most important indicator is the assessment of quality of life of life years gained, which is estimated by a separate observation of survival and quality of life.

As economic evaluation generally requires lifelong follow-up, a modelling approach may be necessary to predict lifelong consequences. By specifying a simulation model or a MARKOV model defining various states characterised by health status and/or specific treatment (associated with a cost-profile) the follow-up period of the actual clinical trial may be reduced. It will be assumed that each state can be characterised by a unique quality of life. The following disease states may be distinguished: disease-free, partial remission, relapse (symptom free), relapse (symptoms) and terminal state. The measurement and valuation of quality of life of patients in the terminal phase of cancer may be difficult, because these patients may be "off protocol" and difficult to approach. Acceptable compliance rates require motivation of the patient, sophistication of the procedure and a permissive attitude towards the ideal interview schedule.

It should be obvious from the above discussion that quality of life measurements are not easy to perform nor to interpret. Drummond (1991) e.g. includes a thorough discussion of the (dis)advantages of such measurements; e.g. the quality of life estimates can only be as 
reliable as the underlying mortality and morbidity data, when different measurement methods may yield different results, and QALYs value quantity (life years) and quality of life in an identical way. Despite the methodological and practical difficulties of outcome measurement, such measures can clearly contribute to improved resource allocations decisions, if applied and interpreted correctly.

\subsection{Measuring and valuing resources}

\subsubsection{Introduction}

The basic idea of economic evaluations is that resources are limited and have an alternative use. Resources used for a specific cancer treatment, e.g. radiation for breast cancer patients, could be used in other cancer treatments, in non-cancer treatments within the health care sector or even outside the health care sector. The true economic cost for a resource is the value this resource would have in its best alternative use, i.e. the opportunity cost. The monetary cost is made up of two components: volume (quantity) times unit price (valuation). In most cases the market price of a resource is the relevant value in monetary terms, but in some cases the price does not correctly reflect the value of the alternative use; sometimes market prices do not even exist. However, a non-existing market price does not mean that the cost is zero; a "shadow price" which reflects the value of the resource in it's best alternative use has to be used.

When valuing and reporting the costs it is useful to identify the quantity of the resources used, and the price separately, before reporting the cost (quantity times unit price).

\subsubsection{Types of costs}

In identifying the relevant costs, it is useful to make a distinction between direct and indirect costs. Traditionally, direct costs are defined as the resources involved in the treatment of the disease. Typical direct medical costs include costs of staff time, drugs, equipment and buildings. However, usually more resources are associated with the treatment than pure medical costs controlled by the health care sector. Direct nonmedical costs such as the patient's travel costs, housekeeping costs, extra diet costs and extra costs for the patient's family are relevant costs that ideally should be incorporated. The importance of nonmedical costs varies between treatments and patient groups. For example, in cost comparisons between home care and hospital care for terminally ill 
cancer patients it is important to include nonmedical costs. As home care requires participation of the patient's family in the treatment process, there are good reasons to assume that nonmedical costs to a larger extent are associated with home care. Indirect costs represent resources lost due to the treatment and illness. Traditionally, indirect costs are valued as the changes in the productivity of the patient in the labour market, i.e. the human capital approach. This means that the indirect costs are estimated as the value of the goods and services not produced due to cancer. However, there are good reasons to include changes in the productivity of leisure activities as well; otherwise time costs for patients who do not participate in the labour market, e.g. retired people, children and housewives, are estimated to be nil. By using the willingness to pay approach these time costs can be estimated, either by explicitly asking individuals how much money they are willing to spend for achieving a better productivity on leisure activities, or if possible, by deriving the value by observing their behaviour (revealed preferences).

The indirect costs primarily stem from changes in the patient's health status. The patient's quality of life changes due to changes in labour productivity and leisure activities might, at least to some degree, reflect the effectiveness of the intervention measure. Hence, there is a risk that productivity changes will be included on the cost side as well as on the outcome side. In order to avoid double-counting indirect costs have to be handled with care.

When presenting cost data it is useful to make a distinction between fixed and variable costs. Fixed costs do not change with the volume of the activity (e.g. the number of procedures and patients), while variable costs do. In identifying the costs per unit of activity (e.g. per patient), it is further necessary to distinguish average costs from marginal costs. The marginal cost refers to the extra cost of 'producing' (e.g. treating) one additional unit (e.g. patient). In situations where the fixed costs are all relatively high the marginal cost for an extra patient will be much heavier than the average cost per patient (e.g. in radiotherapy).

\subsubsection{Perspective and scope of costing}

The costs so far have been classified from the type of resources. However, the classification can be performed from the perspective of who pays. The perspective is important, because the costs for different actors might vary. In a traditional CBA which takes 'society as a whole' as the viewpoint, all costs, direct as well as indirect, should be 
included regardless of who pays and regardless of who benefits. From this viewpoint a treatment is "profitable" if the total benefits exceed the total costs.

In order to correctly calculate the relevant costs of a health care intervention, the viewpoint of the analysis should first be identified. However, other perspectives may be relevant. From the perspective of national health care system the objective is to allocate resources to achieve the maximum health benefit within the limits of resources available for the health care sector. Costs of resources other than those controlled by the health care system, are not included. From the hospital point of view only costs for resources paid by the hospital are relevant; the insurer only takes account of costs charged to him and from the patient's viewpoint only the costs for the patient are of interest. Which viewpoint is relevant, depends upon the aims of the study. Obviously, if the perspective is not clearly identified, cost data may be used in a misleading way.

The fact that a cost is not paid by, for example, the hospital does not mean that the cost is zero. Too narrow a perspective might lead to suboptimal health care resource allocation decisions.

However, the societal perspective is not without controversy either. In most countries the provision of health care is also guided by ideas of justice and equity which implies for example that priority setting must not be based on the patients socioeconomic status. However, the amount of indirect costs obviously depend on the status of the patient. This is hence an argument for excluding the indirect costs and taking into account only the costs paid by the national health care system, i.e. the perspective is limited only to one sector. These considerations underline the need for a systematic presentation of the data so that others can analyse the data in different ways.

\subsubsection{Time scale}

When calculating the costs of a health care intervention, it is important to take a sufficiently long time span into account. Many cancer patients sooner or later relapse and will need further treatment. Ideally, the time span should be long enough to include the costs of all these therapies; preferably, the patients should be followed until death. Too short a time span can lead to doubtful or incorrect conclusions, since part of the relevant costs, on which conclusions should be based, would be omitted. Obviously, it is not always possible, nor feasible, to take such a long time span, but one should be aware of the possible erroneous conclusions, based on a short duration. 


\subsubsection{Collecting data}

Cost-effectiveness studies most frequently assemble information on the resource implications of interventions by 1 . retrospectively using data from individual RCTs, literature surveys, or epidemiological data and clinical advice to derive probabilities attached to treatment pathways and events along them; 2 . collecting descriptive resource data from previous costing studies or published unit cost averages, which enable these pathways and events to be costed; and 3. valuing the identified resource implications of each treatment path and associated probabilities.

It is possible to collect economic data prospectively in a RCT, and to perform economic analysis alongside the clinical and epidemiological aspects of the trial. The potential benefits of doing so have been set out by Drummond and Stoddart (1984) and by Mugford and Drummond (1989). They argue prospective collection of cost information may be desirable if the resource consequences of the treatment gaining widespread acceptance are likely to be substantial; if diffusion is thought likely; or if resource constraints are likely to force decision makers into choices between alternatives. Using these three criteria, Mugford and Drummond found that almost one-half of 100 perinatal trials sampled should have, but did not incorporate, economic analysis.

The main difference between retrospectively collected and prospectively collected data is that prospectively collected data offers better opportunities to examine variations in cost between patients, and therefore to report the distribution of cost data, thus allowing opportunities to examine the statistical significance of cost differences between patient groups.

To ensure consistency and comparability of cost data between study settings, a number of steps should be taken:

a. where possible, resource data should be presented in the form of volumes, such as in-patient days, number and length of consultations, or drugs consumed and prices separately. Summary measures such as drug-days (number of drugs $x$ number of days on drugs) are more useful than no information. Where space does not allow presentation of such data, authors should still make clear that it is available. Such volume data facilitates comparison between practice settings.

$b$. where these resources are valued, the basis of this should be made clear. In particular, it should be stated whether valuation is based on prices charged or on actual resource costs; if the perspective of the analysis is that of the health care financing institutions, 
charges are relevant, but if the viewpoint of the hospital is taken, it is wrong to 'approximate' the cost of an intervention with charge data. In this situation, the actual costs of the resources used should be calculated; whether any consumption taxes such as value added tax are included or excluded; which year and currency the values are expressed in; and the basis for any adjustment in the reported year (for example, whether a consumer price index or some other form of index was used to inflate or deflate values).

c. if resources are valued in a currency other than the currency of the territory in which the study was performed, or if the study covers more than one territory, the method used to translate valuations into other currencies (in particular, the date and basis such as exchange rate or GDP purchasing power parity) should be explicit.

d. where cost-effectiveness ratios are being reported, the numerators and denominators as well as the ratios should be attached to the results.

\subsection{Review of current practice}

\subsubsection{Economic evaluation - a general review}

Current concerns over exploding health care costs and scarce resources have led to a steady increase in studies wholly or partly containing economic evaluations. Elixhauser $e t$ al. (1993) counted a total of 88 CBAs and CEAs carried out in 1979. By 1990 the annual average was 247 with the overall $1979-1990$ total being 1802 studies. This increase on the one hand has led to attempts at cataloguing economic studies in databases and on the other it attempts to develop methods of systematically reviewing a group of studies on the same subjects and of generalising their results. Backhouse et al. (1992) have published the content of their database, containing 1887 economic studies published between 1964 and mid-1992. A similar database has also been recently published by Elixhauser et al. (1993) for the period 1979-1990. Both publications provide a very useful starting point for any search of the economic literature as they incorporate subject and author indices.

Such a plethora of available studies would on the surface indicate an adequate and reliable coverage of the main topics of medical practice. To assess such qualities, however, a systematic review of the topic is needed, but at present there are very few such reviews of economic evaluations. Those that exist all point to considerable gaps in the overall reliability of methodology used in available economic evaluations. 
Gerard reviewed 51 CUAs carried out in 14 different countries and found source validity in only $41 \%$ of the studies (Gerard, 1992). In the discussion of her study Gerard expressed doubts as to the methodological validity of aggregating results of CUAs in so-called QALY league-tables, a process which has the aim of indicating "best buy" procedures for clinicians and managers. Gerard's doubts are shared by Mason et al. (1993) and Drummond et al. (1993I).

This far from satisfactory picture is further confirmed by another study which carefully reviewed methods used in 77 economic analyses with different subjects (Udvarhelyi et al., 1992). The authors recommended that readers should be careful in interpreting results and should scrutinise methods used carefully. Such a suggestion however is unlikely to be ideal for busy clinicians with no training in health economics.

Poor technical execution was also found by Adams et al. (1992) in the crucial area of economic analysis nested within RCTs. Such a finding raises the real problem of the waste of scarce resources in ill-conceived and poorly executed economic evaluations and of a clear requirement to standardise international economic methodology (Drummond et al., 1993). Additionally, only $0.2 \%$ of RCTs contained economic analyses. Jefferson and Demicheli reviewed 90 published and unpublished studies on the economics of introducing vaccination against Hepatitis B (Jefferson and Demicheli, 1994). They found that $92 \%$ recommended vaccination on economic grounds, but a sizeable minority (38\%) showed major methodological inconsistencies. The authors also found very wide variations in basic variables used in the reviewed studies (for instance, the within-country incidence of Hepatitis B used to assess the economic burden of the disease in the same population and the application of basic rules of economic evaluation). An example of the confusion on the subject is given in Table 2.1 where the spread of costs due to Hepatitis B is shown, converted in US Dollars and adjusted to 1993 values using the Retail Price Index. Such a large variation is unlikely to have been caused wholly by different patterns of resources used.

The table also illustrates a possible way of attempting to pool results from different economic studies in order to reach a verdict on whether an intervention is economical or not. At present, efforts are being made to develop such a methodology further (Mugford et al, 1994). 
Table 2.1. Cost per case due to Hepatitis B infection, summary descriptive statistics.

All costs are in US Dollars adjusted from year of publication to 1993 values using the US Retail Price Index (Jefferson and Demicheli, 1994).

\begin{tabular}{|l|r|r|r|}
\hline \multicolumn{4}{|c|}{ Type of cost } \\
\hline & \multicolumn{1}{|c|}{ Direct } & \multicolumn{1}{|l|}{ Indirect } & Indirect intangible \\
\hline Number of studies & 36 & 20 & 11 \\
25th percentile & 650 & 1843 & 14164 \\
Median & 1793 & 3584 & 54272 \\
75th percentile & 3768 & 12473 & 127592 \\
\hline
\end{tabular}

There is probably only one overview on the economics of cancer literature where an attempt has been made at generalising results of different studies (Brown and Fintor, 1993). In this overview Brown and Fintor examined the cost-effectiveness of screening for breast cancer and used a computerised model to 'reconcile' differences in cost-effectiveness ratios from two studies. Encouragingly, they concluded that cost-effectiveness estimates for breast cancer screening reported by different studies are not as arbitrary as they seem.

In summary, although the science of systematic reviews of economic evaluations is in its infancy, the current methodological validity of international literature is open to question and readers should beware of uncritically accepting results from published works without prior scrutiny by a health economist.

\subsubsection{Review of studies specific to cancer}

As discussed above, there has been an increase in the number of studies on the cost-effectiveness of cancer interventions; be they screening, treatment or prevention. This section provides a critical review of the methodological standards of a selected number of recent studies pertaining to a specific common disease area, namely cancer of the bronchus and a specific intervention type, namely screening. This part focuses on studies which include both elements of costs and of effectiveness. The purpose of this review is thus to highlight methodological inadequacies in these studies, using the checklist of guidelines discussed in Section 2.2.3 as a reference. They are selected examples of problems and the strengths of the studies have not been highlighted because of this. 


\section{a. Economic evaluations of treatment for lung cancer}

After a fairly detailed literature search only a few studies concerned with the treatment of lung cancers were identified. The majority of these studies pertained to chemotherapy treatment although one concerned the cost-effectiveness of radiotherapy treatment and two concerned the staging of lung cancer. The study methods and results are outlined in Table 2.2. Whilst these studies have made a major contribution to the literature, there is a need for improved data. Since this chapter is critically examining the use of economic evaluation in cancer the rest of this section concentrates on the weaknesses of these studies. The major methodological failings of these studies are as follows.

- The evidence of the effectiveness does not always appear appropriate. The preferred source of evidence of the effectiveness of interventions is results obtained from a large randomised clinical trial. Most of the selected studies obtained evidence of the effectiveness of interventions from RCTs (e.g. Jaakkimainen et al., 1990; Goodwin et al., 1988). However, in other studies it is unclear from where evidence of the effectiveness of the alternatives evaluated was obtained (e.g. Rees, 1985). Even when data is from RCTs it is not clear whether this is representative of the literature.

- Not all relevant costs of treatment are included in some studies thus questioning the reliability of study results. In an economic evaluation all the costs relating to treatment should be considered. What the relevant costs are depends on the perspective of the study. However, in the study by Dillman et al. (1990), both the costs of treating side-effects and of follow-up care are excluded. This may be of importance, as the treatment which was found to be cost-effective did involve greater hospitalisation due to side-effects.

- Certain studies did not include all relevant consequences, especially the effect of treatment on the quality of life of individuals. Quality of life is often inappropriately omitted from economic evaluations of cancer therapies e.g. in the study by Rees (1985) of the cost-effectiveness of various cancer therapies including palliative care for carcinoma of the bronchus, the benefit from treatment is measured solely by the prolonging of life and the effect of treatment on the quality of a patient's life is disregarded. Similar concerns exist over other studies (e.g. Dillman et al., 1990 and Jaakkimainen et al., 1990), as the omission of quality of life considerations questions the validity of study results. However, the study by Goodwin et al. (1988) does illustrate how the credible valuation of the quality of life of patients can be incorporated within a study. Quality of life is all the more important since the great majority of treatment aims at palliation rather than cure. 
Tabel 2.2 Economic evaluations relating to lung cancer

\begin{tabular}{|c|c|c|c|c|}
\hline Ref. & $\begin{array}{l}\text { Country } \\
\text { and } \\
\text { analysis }\end{array}$ & Study area & $\begin{array}{l}\text { Alternatives } \\
\text { evaluated }\end{array}$ & Main results \\
\hline $\begin{array}{l}\text { Dillman } \\
\text { et al. } \\
(1990)\end{array}$ & $\begin{array}{l}\text { USA } \\
\text { CEA }\end{array}$ & $\begin{array}{l}\text { Induction chemo- } \\
\text { therapy plus radio- } \\
\text { therapy for patients } \\
\text { with non-small cell } \\
\text { lung cancer. }\end{array}$ & $\begin{array}{l}\text { Radiation plus } \\
\text { chemotherapy } \\
\text { vs radiation } \\
\text { alone. }\end{array}$ & $\begin{array}{l}\text { The inclusion of induction } \\
\text { chemotherapy leads to a } \\
33 \% \text { increase in the costs of } \\
\text { therapy but a } 100 \% \text { increase } \\
\text { in mean survival after } 2 \text { and } \\
3 \text { years. }\end{array}$ \\
\hline $\begin{array}{l}\text { Eddy } \\
(1990)\end{array}$ & $\begin{array}{l}\text { Canada } \\
\text { CMA }\end{array}$ & $\begin{array}{l}\text { Staging of lung can- } \\
\text { cer - evaluating the } \\
\text { mediastinum before } \\
\text { surgery. }\end{array}$ & $\begin{array}{l}\text { CT scan vs no } \\
\text { CT scan. }\end{array}$ & $\begin{array}{l}\text { The approach of using a CT } \\
\text { scan to determine the need } \\
\text { for mediastinoscopy with } \\
\text { nodal biopsy rather than } \\
\text { giving these tests routinely } \\
\text { reduced the costs of treating } \\
\text { these patients. }\end{array}$ \\
\hline $\begin{array}{l}\text { Good- } \\
\text { win } \\
\text { et al. } \\
\text { (1988) }\end{array}$ & $\begin{array}{l}\text { Canada } \\
\text { CUA }\end{array}$ & $\begin{array}{l}\text { Treatment of exten- } \\
\text { sive small-cell Jung } \\
\text { cancer. }\end{array}$ & $\begin{array}{l}\text { Chemotherapy } \\
\text { regimes. }\end{array}$ & $\begin{array}{l}\text { The most expensive regimen } \\
\text { (cyclophosphamide, } \\
\text { vincristine and doxorubicon) } \\
\text { was more CE, as it was } \\
\text { more effective and savings } \\
\text { were made in other resour- } \\
\text { ces. }\end{array}$ \\
\hline $\begin{array}{l}\text { Houston } \\
\text { et al. } \\
\text { (1985) }\end{array}$ & $\begin{array}{l}\text { USA } \\
\text { CMA }\end{array}$ & $\begin{array}{l}\text { Staging of lung can- } \\
\text { cer - evaluating the } \\
\text { mediastinum and } \\
\text { assessing metastases. }\end{array}$ & $\begin{array}{l}\text { CT scan ys no } \\
\text { CT scan. }\end{array}$ & $\begin{array}{l}\text { CT scans appeared to be no } \\
\text { more accurate than current } \\
\text { routine tests and the use of } \\
\text { CT scans was more expen- } \\
\text { sive. }\end{array}$ \\
\hline $\begin{array}{l}\text { Jaakki- } \\
\text { mainen } \\
\text { et al. } \\
(1990)\end{array}$ & $\begin{array}{l}\text { Canada } \\
\text { CEA }\end{array}$ & $\begin{array}{l}\text { Treatment of } \\
\text { advanced non-small } \\
\text { cell lung cancer. }\end{array}$ & $\begin{array}{l}\text { Chemotherapy } \\
\text { regimes vs best } \\
\text { care. }\end{array}$ & $\begin{array}{l}\text { Both drug regimes were CE } \\
\text { when compared to best care. } \\
\text { The less expensive regime } \\
\text { (cyclophosphamide, doxo- } \\
\text { rubicin and cisplatin) was } \\
\text { more CE and cost saving. }\end{array}$ \\
\hline
\end{tabular}




\begin{tabular}{|l|l|l|l|l|}
\hline Ref. & $\begin{array}{l}\text { Country } \\
\text { and } \\
\text { analysis }\end{array}$ & Study area & $\begin{array}{l}\text { Alternatives } \\
\text { evaluated }\end{array}$ & Main results \\
\hline $\begin{array}{l}\text { Pashko } \\
\text { et al. } \\
(1992)\end{array}$ & USA & $\begin{array}{l}\text { Chemotherapy treat- } \\
\text { ment in small cell } \\
\text { lung cancer. }\end{array}$ & $\begin{array}{l}\text { Chemotherapy } \\
\text { regimes. }\end{array}$ & $\begin{array}{l}\text { The regime involving the } \\
\text { oral administration of a } \\
\text { larger dose of otoposde on } \\
\text { study days 2 and 3 rather } \\
\text { than a summer intravenously } \\
\text { administered dose was less } \\
\text { costly. Both regimes were of } \\
\text { equal efficacy. }\end{array}$ \\
\hline $\begin{array}{l}\text { Rees } \\
(1985)\end{array}$ & UK & $\begin{array}{l}\text { Palliative treatment } \\
\text { for carcinoma of the } \\
\text { bronchus. }\end{array}$ & $\begin{array}{l}\text { Radiotherapy } v s \\
\text { no intervention. }\end{array}$ & $\begin{array}{l}\text { Palliative care had a higher } \\
\text { cost per "notional patient } \\
\text { benefit year" than curative } \\
\text { therapies for other cancer } \\
\text { therapies. }\end{array}$ \\
\hline
\end{tabular}

- The methods used by most of the published studies to measure and value resource use are not ideal. Most of the studies reviewed adopt a methodology similar to Pashko and Johnson (1992), whereby the average use of resources attributable to a procedure are measured and an average cost per unit for each form of resource employed is calculated. An average resource cost per procedure can then be estimated. In the study by Rees (1985), the methods for the measurement of resource use are not made clear. An alternative, more accurate, method is to monitor a sample of patients throughout their course of treatment (either prospectively by the use of case record forms, or retrospectively by examining hospital charts), measuring resource use as it is employed and from this analysis derive a marginal cost for the procedure. The studies by Houston et al. (1985) and Eddy (1989) adopted this approach.

- In almost all of the studies selected there is a failure by authors to include any analysis of the sensitivity of their results. However, the studies by Goodwin et al. (1988) and Jakkiminen et al. (1990) include several such analyses.

\section{b. Economic evaluations of cancer screening programmes}

There are relatively few comprehensive economic evaluations of cancer screening programmes or policies. Most pertain to the area of breast cancer screening, partly due to 
the important body of literature on randomised and case controlled clinical trials in this field. Economic analyses of cervical cancer or colorectal cancer screening initiatives are more scarce. The majority of economic analyses of breast cancer screening are based on theoretical models, which combine efficiency results from former clinical trials with actual costs of screening programmes or clinical practice. Analyses of cervical and colorectal cancer screening programmes either base estimates on theoretical models or are limited to very small numbers of cases. Among the studies presented in Table 2.3, the following remarks need to be addressed regarding to methodology:

- The evidence for effectiveness is not always based on RCTs and, if so, the results of RCTs are not readily applicable to the situation. Joseph et al. (1988), for example, base their measurement of the HemoQuant test's effectiveness in screening for bowel cancer on a sensitivity value obtained in a study of only 30 patients. Obuko et al. (1991) use a combination of sources for estimates of efficiency, yet fail to address the possible discrepancies in the calculation of these estimates. Van der Maas et al. (1989) and De Koning et al. (1991) suitably address the problem of the applicability of clinical trial results by combining results from two respected clinical trials for the measurement of outcome in their analysis. The reliability and applicability of results from clinical trials performed several years prior to the economic evaluation or a screening programme must be assessed with respect to possible changes in technology and in population demographics which could limit their relevance to the current situation.

- A comprehensive description of alternatives is not always provided and rendering interpretation of results and applicability of conclusions are difficult. In their evaluation of cervical screening policies, Parkin and Moss (1986) analyse seven alternative policies in terms of cost-effectiveness, yet the distinction between the alternative policies is at times unclear throughout the text. In the study by Johnson et al. (1987) no alternative situation is discussed. In Mandelblatt et al. (1988) the number of screened patients included in the study is very small and the logical comparison group, non-screened patients of similar age, is excluded from all analyses. 
Tabel 2.3 Economic evaluations relating to cancer screening programmes

\begin{tabular}{|c|c|c|c|c|}
\hline Ref. & $\begin{array}{l}\text { Country } \\
\text { and } \\
\text { analysis }\end{array}$ & Study area & $\begin{array}{l}\text { Alternatives } \\
\text { evaluated }\end{array}$ & Main results \\
\hline $\begin{array}{l}\text { Joseph } \\
\text { et al. } \\
\text { (1988) }\end{array}$ & $\begin{array}{l}\text { USA } \\
\text { CEA }\end{array}$ & $\begin{array}{l}\text { Colorectal cancer } \\
\text { screening. }\end{array}$ & $\begin{array}{l}\text { HemoQuant vs } \\
\text { Hemoccult } \\
\text { screening. }\end{array}$ & $\begin{array}{l}\text { Despite higher sensitivity } \\
\text { of the HemoQuant test, its } \\
\text { high cost is only justified if } \\
\text { it can provide a specificity } \\
\text { similar to that of the Hem- } \\
\text { occult test. }\end{array}$ \\
\hline $\begin{array}{l}\text { Johnson } \\
\text { et al. } \\
\text { (1987) }\end{array}$ & $\begin{array}{l}\text { USA } \\
\text { CEA }\end{array}$ & $\begin{array}{l}\text { Mass colorectal } \\
\text { cancer screening. }\end{array}$ & $\begin{array}{l}\text { Self-administe- } \\
\text { red stool blood } \\
\text { test (no alter- } \\
\text { natives). }\end{array}$ & $\begin{array}{l}\text { The studied programme } \\
\text { costs per cancer detected } \\
\text { are fairly high }(\$ 9670) \text {. } \\
\text { The authors advocate mass } \\
\text { screening programmes to } \\
\text { be targeted towards at-risk } \\
\text { populations. }\end{array}$ \\
\hline $\begin{array}{l}\text { Mandel- } \\
\text { blatt } \\
\text { et al. } \\
\text { (1988) }\end{array}$ & $\begin{array}{l}\text { USA } \\
\text { CEA }\end{array}$ & $\begin{array}{l}\text { Cervical cancer } \\
\text { screening in low- } \\
\text { income, elderly } \\
\text { women. }\end{array}$ & $\begin{array}{l}\text { One-time Pap- } \\
\text { anicolaou test } \\
\text { for cervical } \\
\text { cancer } v s \text { no test } \\
\text { (hypothetically) } \\
\text { in the same } \\
\text { population. }\end{array}$ & $\begin{array}{l}\text { The proposed programme } \\
\text { would both be efficient and } \\
\text { cost-saving, thus suppor- } \\
\text { ting cervical cancer screen- } \\
\text { ing of elderly women. }\end{array}$ \\
\hline $\begin{array}{l}\text { Parkin } \\
\text { and } \\
\text { Moss } \\
(1986)\end{array}$ & $\begin{array}{l}\text { UK } \\
\text { CEA }\end{array}$ & $\begin{array}{l}\text { Cervical cancer } \\
\text { screening. }\end{array}$ & $\begin{array}{l}7 \text { screening } \\
\text { policies recom- } \\
\text { mended in the } \\
\text { UK since } 1966 \\
\text { (using different } \\
\text { screening sche- } \\
\text { dules and tar- } \\
\text { geting different } \\
\text { age groups). }\end{array}$ & $\begin{array}{l}\text { The best CE-ratio obtained } \\
\text { corresponds to screening at } \\
5 \text {-year intervals of women } \\
\text { over } 35 \text {. Extension of } \\
\text { screening to women under } \\
35 \text { leads to loss of effi- } \\
\text { ciency. }\end{array}$ \\
\hline $\begin{array}{l}\text { Mooney } \\
(1982)\end{array}$ & $\begin{array}{l}\text { UK } \\
\text { CEA }\end{array}$ & $\begin{array}{l}\text { Breast cancer scree- } \\
\text { ning, women aged } 40 \\
\text { to } 59 .\end{array}$ & $\begin{array}{l}\text { Mammography, } \\
\text { thermography } \\
\text { and clinical } \\
\text { exam vs mam- } \\
\text { mography and } \\
\text { thermography } \\
\text { ( } 6 \text { alternatives } \\
\text { in total). }\end{array}$ & $\begin{array}{l}\text { Mammography (single } \\
\text { reporting) combined with a } \\
\text { single clinical examination } \\
\text { showed the most attractive } \\
\text { cost-effectiveness ratio of } \\
\text { the alternatives studied. }\end{array}$ \\
\hline
\end{tabular}




\begin{tabular}{|c|c|c|c|c|}
\hline Ref. & $\begin{array}{l}\text { Country } \\
\text { and } \\
\text { analysis }\end{array}$ & Study area & $\begin{array}{l}\text { Alternatives } \\
\text { evaluated }\end{array}$ & Main results \\
\hline $\begin{array}{l}\text { Forrest } \\
(1987)\end{array}$ & $\begin{array}{l}\text { UK } \\
\text { CUA }\end{array}$ & $\begin{array}{l}\text { Mass breast cancer } \\
\text { screening by mam- } \\
\text { mography, women } \\
\text { aged } 50 \text { to } 64 .\end{array}$ & $\begin{array}{l}\text { Mammography } \\
\text { as } 3 \text {-year inter- } \\
\text { vals in women } \\
\text { aged } 50 \text { to } 64 \mathrm{vs} \\
\text { no screening. }\end{array}$ & $\begin{array}{l}\text { Mass screening by } \\
\text { mammography at } 3 \text {-year } \\
\text { intervals would result in } \\
695 \text { QALYs per } 100.000 \\
\text { women invited for scree- } \\
\text { ning. }\end{array}$ \\
\hline $\begin{array}{l}\text { Van der } \\
\text { Maas } \\
\text { et al. } \\
\text { (1989) }\end{array}$ & $\begin{array}{l}\text { NL } \\
\text { CEA }\end{array}$ & $\begin{array}{l}\text { Breast cancer scree- } \\
\text { ning by mammo- } \\
\text { graphy, women aged } \\
50-70 \text { years. }\end{array}$ & $\begin{array}{l}4 \text { alternatives } \\
\text { based on differ- } \\
\text { ent screening } \\
\text { schedules: } 4- \\
\text { year, } 2 \text {-year, } \\
1,3-\text { year, and 1- } \\
\text { year frequen- } \\
\text { cies, are com- } \\
\text { pared to a no- } \\
\text { screening situa- } \\
\text { tion. }\end{array}$ & $\begin{array}{l}\text { Mammographic screening } \\
\text { of women aged } 50-70 \text { at } 2 \text { - } \\
\text { year intervals allows for } \\
\text { savings of } \$ 4850 \text { per life } \\
\text { year saved, a ratio which } \\
\text { compares favourably to that } \\
\text { of screening at either shor- } \\
\text { ter or longer intervals and } \\
\text { to that of other health } \\
\text { interventions. }\end{array}$ \\
\hline $\begin{array}{l}\text { De } \\
\text { Koning } \\
\text { et al. } \\
\text { (1991) }\end{array}$ & $\begin{array}{l}\text { NL } \\
\text { CEA/ } \\
\text { CUA }\end{array}$ & $\begin{array}{l}\text { Mass breast cancer } \\
\text { screening by mam- } \\
\text { mography in women } \\
\text { aged } 50-70 \text {. }\end{array}$ & $\begin{array}{l}\text { The invitation } \\
\text { schedules used } \\
\text { in the Van der } \\
\text { Maas study are } \\
\text { compared for } \\
\text { their respective } \\
\text { effect on quality } \\
\text { of life and } \\
\text { QALYs. }\end{array}$ & $\begin{array}{l}\text { Mass screening of women } \\
\text { aged } 50-70 \text { would appear to } \\
\text { affect quality of life very } \\
\text { little, as the cost per } \\
\text { QALY differs little from } \\
\text { the cost per life year } \\
\text { gained unadjusted for } \\
\text { quality. The authors recom- } \\
\text { mend the adoption of a } \\
\text { national screening policy } \\
\text { based on a 2-year schedule. }\end{array}$ \\
\hline $\begin{array}{l}\text { Eddy } \\
\text { et al. } \\
(1988)\end{array}$ & $\begin{array}{l}\text { USA } \\
\text { CEA }\end{array}$ & $\begin{array}{l}\text { Mass breast cancer } \\
\text { screening by mam- } \\
\text { mography in women } \\
\text { under } 50 \text {. }\end{array}$ & $\begin{array}{l}\text { Annual mam- } \\
\text { mography for } \\
\text { women aged } 40 \\
\text { to } 49 \text { already } \\
\text { having had a } \\
\text { physical exam } \\
\text { is physical } \\
\text { exam alone. }\end{array}$ & $\begin{array}{l}\text { Reduction in the costs of } \\
\text { treatment in screened } \\
\text { women under } 50 \text { would be } \\
\text { outweighed by extra costs } \\
\text { of screening and necessary } \\
\text { biopsies. }\end{array}$ \\
\hline
\end{tabular}




\begin{tabular}{|l|l|l|l|l|}
\hline Ref. & $\begin{array}{l}\text { Country } \\
\text { and } \\
\text { analysis }\end{array}$ & Study area & $\begin{array}{l}\text { Alternatives } \\
\text { evaluated }\end{array}$ & Main results \\
\hline $\begin{array}{l}\text { Obuko } \\
\text { et al. } \\
(1991)\end{array}$ & Japan & $\begin{array}{l}\text { Breast cancer scree- } \\
\text { ning in women 30-80 } \\
\text { years old. }\end{array}$ & $\begin{array}{l}\text { Physical exam } \\
\text { alone (current } \\
\text { Japanese policy) } \\
\text { vs combinations } \\
\text { of mammo- } \\
\text { graphy and } \\
\text { physical exam } \\
\text { vs no screening. }\end{array}$ & $\begin{array}{l}\text { According to the computer } \\
\text { simulation model, scree- } \\
\text { ning by mammography } \\
\text { alone provided the best } \\
\text { (lowest) CE-ratio of the } \\
\text { studied alternatives. }\end{array}$ \\
\hline $\begin{array}{l}\text { Gravelle } \\
\text { et al. } \\
(1982)\end{array}$ & $\begin{array}{l}\text { England } \\
\text { and } \\
\text { Wales }\end{array}$ & $\begin{array}{l}\text { Breast cancer scree- } \\
\text { ning in women over } \\
40 .\end{array}$ & $\begin{array}{l}\text { Physical exam } \\
\text { + mammo- } \\
\text { graphy vs con- } \\
\text { ventional man- } \\
\text { agement (no } \\
\text { Screening). }\end{array}$ & $\begin{array}{l}\text { The authors conclude that } \\
\text { the introduction of scree- } \\
\text { ning would increase costs } \\
\text { for the NHS, yet the cost } \\
\text { per life year saved is very } \\
\text { low. }\end{array}$ \\
\hline
\end{tabular}

- Economic studies of screening policies often fail to include all relevant costs of screening (especially capital costs) and all relevant consequences (especially morbidity). Little consideration is given to the effect on patients' quality of life. Indeed, the only study which incorporates quality of life measurements is the study by De Koning et al. (1989) on breast cancer screening, all other studies being of cost-effectiveness. The study by Joseph et al. (1988) considered mortality as the only possible negative consequence of Hemoccult and HemoQuant screening, and limited costs to the charge for the screening procedure, excluding all capital costs. Johnson et al. (1981) took the total cost of the programme and divided it by the number of cancers detected in the study group to obtain a cost-effectiveness ratio. Inadequacies in the estimation of costs and outcomes question the credibility of economic evaluations.

- Costs and consequences are not always adjusted for differential timing. Most of the studies in Table 2.3 include appropriate discounting of costs and consequences of screening, although no discounting is done in the case of Johnson et al. (1987) and only outcomes are discounted in the study by Parkin and Moss (1986).

- The costs and outcomes of different screening alternatives are rarely studied in the context of an incremental analysis, and only total sums and average costs are presented and discussed. The articles by Van der Maas et al. (1998) and De Koning et al. (1989) 
are two examples of studies which include detailed incremental analysis in their estimates of cost-effectiveness.

- The discussion in these papers presents the authors' recommendation for screening, yet fails to discuss its implementation or to place the study results within the context of health care priorities. Since the goal of economic evaluation is to aid policy makers in choosing among competing policies, the conclusion of most studies either advocates in favour of or against the implementation of a given screening policy. However, very few studies mention which difficulties might arise in the implementation of policies, or which competing policies should also be analysed in terms of cost-effectiveness. An example can be found in Mandelblatt's recommendation to implement cervical cancer screening programmes for elderly women (Mandelblatt et al., 1988). The author never addresses the issue of cervical screening in younger women (under 70 years) and neither does she compare the cost-effectiveness ratio in women over 70 years to that obtained in studies of younger women.

Other, also methodological problems with breast cancer screening studies are discussed further by Skrabanek (1991).

\subsection{Use of results of economic evaluations}

\subsubsection{Use by policy makers}

The policy maker's aim is that of choosing and implementing a "best-buy" strategy to deal with a particular aspect of cancer, be it prevention, treatment or care for cancer patients and their families. This is particularly important in the field of neoplastic diseases which tie up a considerable amount of resources. Such a requirement is especially important when the policy maker is in a purchasing role and must make best use of available resources in commissioning a new service or must look at making best alternative use of available resources.

If the decision making process is to be of good quality it must be based on reliable assumptions. If the reliability of international economic literature is open to question, where should the decision maker go for advice? Probably the quickest way of getting such advice is to ask an economist, in other words somebody who not only has the technical knowledge required to provide sound advice, but also whose everyday work is economic evaluation. If not involved in the specific field of enquiry a local economist should be 
able to direct the enquirer to economists active in the field. Alternatively, some national or international organisations are beginning to develop a strategy to review and disseminate results of economic evaluations. These include the Cochrane Collaboration through Centres in Oxford, Copenhagen, Ontario and other international centres, and the University of York in the United Kingdom and the offices of Health Technology Assessment, being established in many countries (e.g. Sweden, Canada and Australia).

\subsubsection{Use by clinicians}

The results of economic evaluation of therapies should not be regarded as being simply in the domain of planners and 'purchasers'. In our cost-constrained world decisions on health care spending are going to be everyones business. As expensive new therapies develop health care planners are not always going to make hard decisions on whether to pay for them. Often they will allow clinicians to use such treatments, provided that they are paid for out of the current budget. Clinicians will, in this situation, need to understand any available economic evaluation and be able to compare it with economic evaluations of other therapies they provide. Only then they will be in a position to decide which treatments to use. In cost-constrained health systems management does not have sufficient expertise or manpower to make decisions based on economic evaluations in the myriads of interventions in many areas of medicine. Inevitably this role will often be devolved to the individual clinicians or departments.

\subsubsection{Use of the results of economic evaluations}

Comprehensive cost-effectiveness studies can be expensive to perform, and may be hard to repeat if attached prospectively to a large randomised controlled trial. Given the comparatively small number of studies performed each year, and the likelihood that their results will have a limited lifetime before being overtaken by changes in technical or allocative efficiency or by technical progress, it is important to find ways of making maximum use of their results. The transferability of the results of a cost-effectiveness study may be considered in a number of dimensions:

a. The perspective of the study: A study may have been performed from one of a number of perspectives, such as that of a third party payer, the health sector, the public or private sub-component of the health sector, or society as a whole. This may restrict the transferability of the results to another setting with different divisions of responsibility or with 
different information requirements. Transferability would therefore be facilitated by reporting, where possible, the results from a standard perspective, which may be the baseline perspective adopted by the study or a supplementary part of the analysis. The health sector as a whole would probably raise fewest problems of transferability although also this perspective may pose problems at least for studies in e.g. the field of terminal care or home care, since whether such types of care fall under the 'health care' or 'social services' may vary widely between countries. Further work is required in this area before prescriptive guidelines could be agreed.

b. The care level of the study: A cost-effectiveness study performed in a teaching hospital or trial setting may be based on substantially higher unit costs than would be the case in a non-teaching hospital. Therefore some indication of the extent to which the costs reported can be regarded as typical of the health sector as a whole would be useful.

c. Regional differences: Similarly there may be regional differences in costs within a country that result in the reported results being typically low or high for the country as a whole. Some indication of this would also be useful.

d. The time the study was performed: A cost-effectiveness study may make use of information on costs, treatment paths and outcomes, all from different sources and potentially different times. These data, and in particular the technology and organisation of care in the treatment paths, must be made explicit in order that obsolescence of the study can be judged.

e. The country of the study: To facilitate wider use of the study, it may be tempting to consider ways of transferring results from one country to another. To facilitate this, it is essential that the guidelines set out above in section 2.4, as well as items a-d on transferability are closely adhered to. Efforts to achieve this are in their infancy and more effort is needed on the methodologies and empirical procedures relevant to international transferability.

\subsection{Recommendations}

We have the following recommendations:

a. When any clinical trial is being planned the potential economic outcome of the intervention should be considered. The requirement for economic evaluation should also 
be examined. Even when this is considered inappropriate thought should be given to collecting basic data which might be used for a later economic evaluation.

$b$. If an economic evaluation is being planned the advice of a health economist should be sought at the outset.

c. Funding for RCTs is limited. Ask yourself is the design efficient, is it likely to answer the question or make a useful contribution, would the money be better spent elsewhere?

d. Do not try to replicate locally an economic evaluation study you have found in the literature without involving a trained economist. Listings of health economists will be available from national Offices of Health Technology Assessment and in Europe from the EORTC office in Brussels.

$e$. Where possible, resource data should be presented in the form of volumes, such as in-patient days, number and length of consultations or drugs consumed. After the identification of the cost of each of the resources, the costs can be calculated. Authors submitting cost-effectiveness studies should ensure that, where available, results report ranges and confidence intervals around the cost data used, and that sensitivity analyses on the main dimensions of the study are performed and reported. Results in the form of a single point estimate of cost-effectiveness for a given intervention should be discouraged. Numerators and denominators of cost-effectiveness ratios should be reported.

$f$. There is a need for dialogue between clinicians, policy makers and health economists. The present gap is liable to lead to inappropriate health evaluations and ill informed discussion. The end result may be poorer health care for patients.

g. There is a need to ensure methods used in studies. In all published studies, the methods used in measurement and valuation of all costs and consequences must be made clear - to allow both policy makers to determine whether the study's results are applicable to their policy arena and to help other researchers avoid duplication of research and to facilitate the extrapolation of studies to other settings. However, this has to be balanced with editors' desires to constrain the space available to report such studies. Often when researchers are criticised for not accurately displaying the methods adopted this is the result of editors being parsimonious with the space available to present the work and not with any desire on behalf of the authors to conceal their methods. 
Editors and authors must consider how to make a detailed description of methods employed more available. Methods could be made available by being published as an appendix to the main article. However, given space constraints this is an unlikely option. As the trend to make journals available online gathers pace, one alternative available is to include within the online version of the journal details of the methods employed. Another alternative is for editors to insist that authors make available a full description of the methods to all those requesting them. Even if the methods are not contained in the main body of text within their journal, they must still be made subject to peer review. There is a need for a European Workshop to discuss standardisation of method and reporting with a special emphasis on transferability.

$h$. Editors should ensure that a health economist is included among referees for papers containing an economic evaluation component.

i. Coordination of teaching and education: In order to achieve optimal understanding and cooperation between health economists, clinicians and policy makers, each party must be able to appreciate the objectives, methodology and priorities of the others' vision of the health care sector. This mutual understanding and respect can be achieved by integrating elements of health economics into medical school curricula and similarly by giving health economics students basic medical concepts. On a European Community level, a forum for dialogue between all parties can be achieved in the form of symposia, guidelines recommending the inclusion of economic aspects in evaluations of oncological interventions, or encouragement of multidisciplinary approaches in cancer care research.

$j$. Economic evaluation of cancer care is complicated by the poor quality of the evidence currently available on treatment efficacy. The lack of data on quality of life and symptom control further complicates assessment of efficacy in what is often palliative therapy.

$k$. Analyses of the cost-effectiveness in cancer care must be based on economic evaluation studies. As there are so far few studies of good quality the information and knowledge from existing studies is of great importance. Where possible health economics should provide information on on-going studies to a central database. The $\mathrm{EC}$ has an important role to encourage this and, especially by overcoming the problems of recognising and translating non-english language papers.

l. Health policy makers should be considering the need for an economic analysis of any new expensive therapy. They may choose only to recommend such therapies for licensing and wide spread use if this economic evaluation is favourable. 


\section{Economic evaluation alongside cancer trials}

\subsection{Summary}

A recent extension of clinical evaluation is "economic evaluation", which seeks to characterise each relevant alternative health care strategy in terms of a summary measure incorporating the costs and benefits of such strategies. In an economic evaluation, separate measurements of resource volumina and resource prices on the cost side, and separate measurements of survival and quality of life effects and valuation of these outcome effects on the benefit side are required. From these effect parameters, which should be calculated for all competing strategies considered in the analysis, the relative cost-effectiveness of one strategy as against the other can be derived. The degree of generalisability of the study results determines the validity of economic evaluation in decision making. This depends on the generalisability of the clinical findings, and in this respect the so-called "piggyback" economic evaluation, which is added to a clinical trial, has its limitations. In the field of cancer, specific attention should be given to costs and effects occurring after non-mortality end points, to patient and family costs and to variations in treatments between settings of care. It is argued that conventional clinical trials and economic evaluations will integrate further in the future.

\subsection{Introduction}

Cancer not only accounts for a major burden of mortality and morbidity (about one third of all mortality in the Netherlands, for example), its economic impact is also considerable (Koopmanschap et al., 1991; Broder, 1991; Yarbro, 1991). During the last few decades, fundamental and clinical research have provided a better understanding of cancer, and as a result improved prognosis (survival, quality of life) of patients can be observed. These results have been achieved through the development of refined diagnostics, new surgical 
techniques and new drugs, but also through rigorously carrying out clinical experiments and thoroughly analysing their results. One of the methodological innovations has been the introduction of quality of life outcome measures in clinical evaluation research, in addition to the use of standard fixed year survival rates. Moreover, the apparent trade-off between survival prolongation and quality of life improvement has urged the development of combined mortality-morbidity measures (Goldhirsch et al., 1989). Economic evaluation is an even more recent development which broadens the scope of evaluation (Drummond et al., 1987(I); Drummond et al., 1987(III)). It has been induced by increased awareness of limited health care resources, and the consequent need for economically and medically sound choices. Particularly in the case of cancer, the economic issue is relevant (Yarbro, 1991; Heine and Rothenberger, 1991; Berg, 1991) as the costs per case are usually high, and expected benefits are still small for large groups of patients (Hancock, 1992). This article describes some theoretical aspects of economic evaluation and some practical issues of economic evaluation are combined with a clinical trial (Drummond and Davies, 1991). Its application in oncology is illustrated.

\subsection{Economic evaluation: theory}

\subsubsection{Theoretical foundation}

If costs have to be taken into account in clinical decision making, the problem arises of how to incorporate this argument. Apparently, additional data and new definitions of the optimal choice are needed. To understand the peculiarities of economic evaluation as more than merely an additional technique in epidemiology requires some notion of its theoretical background. From an economic point of view, health care resource allocation is rather inefficient. Policies directed to more efficiency appear to be necessary, and have to be guided by systematically collected and integrated information on the costs and benefits of health care. The traditional economic instrument for evaluating alternative public sector initiatives in this way is cost-benefit analysis (CBA), which is founded in Paretian welfare economics. This theory defines a situation as "efficient" if no potential Pareto improvement is possible, the latter implying that at least one person can be made better off and no one worse off if the losers are compensated from the beneficiaries' gains (Mishan, 1984). In this definition, the individual's own utility or willingness to pay is decisive, which poses some restrictions on the use of CBA when other values are deemed important, as is the case in health care (Culyer, 1991). Thus, a broader framework is needed in health care with the same goal in mind: the determination of a unique preferential ordering of (health care) programmes (Drummond, 1992). This broader framework 
again starts from quantification of all costs and benefits but the measurement of benefits is adapted. Instead of monetary units, such as the willingness to pay, non-monetary units may be used. In standard cost-effectiveness analysis (CEA), the benefits are expressed in one appropriate natural unit as, for instance, life years saved. CEA is often used to describe this whole cluster of economic evaluation approaches. More recently, cost-utility analysis (CUA) has emerged, which applies so-called utility values which aggregate different dimensions of benefits. Note that the latter approach is particularly relevant in the case of oncology, where we often meet different arrays of effects under different therapeutic options. Benefits measurement in economic evaluation in trying to cover all relevant outcome aspects is not new, but it specifically aims at summary values which can bear the claim that they represent overall societal preference values.

\subsubsection{Choice of perspective}

The preferred choice of perspective in CEA (as in CBA) is that of "society", as opposed to, for example, "patient" or "insurance company". This perspective implies that all costs and benefits for all parties in society should be accounted for. Some of the less obvious consequences of this perspective are listed below. First, patient costs (both out of pocket expenses and time-investments) should be included. This theoretical point is relevant in patients with cancer as they, for example, frequently apply dietary measures and spend much time in diagnosis and treatment. For similar reasons, the care by close relatives or friends is not zero-priced, but appropriately valued. In oncology, we frequently observed the erroneous assumption that non-professional care of terminally ill patients by volunteers and close relatives is a preferable option because it is zero-priced. Second, the lost production due to illness or treatment is also a cost to society, and the associated indirect costs should be included in the analysis (for a description of techniques see Koopmanschap and Van Ineveld, 1992). Indirect costs in cancer are usually substantial. Third, accepting society's perspective implies that the real use of resources should be measured and valued rather than the charges paid by patients or third parties, as the latter may bear little resemblance to actual costs. This is, for example, relevant in the assignment of costs to days of hospitalisation, laboratory tests and medical services. An example in oncology of a more remote consequence is that economic evaluation uses the expected market price of a new chemotherapeutic drug, even if it may be temporarily zero-priced for research purposes. 


\subsubsection{Choice of approach}

There are several pathways which arrive at the numerical values of the indicators for costeffectiveness. In this paper, we will concentrate on the so-called "piggyback" design, particularly in oncological research. "Piggyback" indicates that the economic evaluation is added on to an established clinical trial as a prospective or retrospective supplement (Drummond and Davies, 1991). If the clinical trial provides insufficient data or a prospective experimental design is not feasible, some secondary options are usually feasible, particularly the synthesis of the major findings from literature within a mathematical model (Martens et al., 1989).

Below we discuss the "piggyback" approach and potential frictions arising from the reconciliation of the study requirements for clinical and economic evaluations. These frictions disclose the different perspectives of clinical versus economic evaluations: the clinical investigator generally seeks to demonstrate effectiveness in a well-defined patient group on the basis of a sensitive (clinical) outcome indicator, while the economist primarily wants to support a policy decision on an intervention or programme by assessing its general cost-effectiveness in routine medical practice, comparing the best alternatives available.

\subsection{Economic evaluation as an add-on to a controlled clinical trial}

Controlled clinical trials (CCT) are increasingly considered as natural vehicles for economic evaluations (Drummond and Davies, 1991). The added costs of an economic evaluation are obviously relatively low, and the importance of economic evidence at an early phase of the health policy process is acknowledged by all health care parties involved (Rutten and Bonsel, 1992). With the resulting closer integration of economists within the clinical trial setting, the following issues have emerged.

\subsubsection{Mode of comparison}

As in a clinical trial, a randomised controlled comparison is preferred in an economic evaluation. However, in some cases the formation of a control group may be difficult for ethical or organisational reasons. For example, the course of disease under conventional treatment may be invariably lethal, making patients reluctant to participate on a randomised basis if a new potentially life saving therapy is offered (e.g. an immune therapy 
versus placebo trial in melanoma). In the absence of a control group, prognostic modelling of control group survival may be an option (Bonsel et al., 1990), but economic evaluation invariably requires a parallel prognostic model for information on the costs in the control situation. In selected cases, another option for obtaining control group estimations may be the extrapolation of waiting list data on survival, quality of life and costs (Van Hout et al., 1993).

\subsubsection{Choice of alternatives}

The choice of alternatives within a CCT determines the suitability of the trial for add-on economic evaluation. If the CCT does not include the usual or the best alternative treatment as a control, an economic evaluation is impossible. Placebo control, often essential in determining efficacy, is only suitable in economic evaluation if the notreatment option reflects the best alternative action. However, in other cases, economic evaluation would sometimes require the placebo or no-treatment option in a CCT where it is not included. An example of the latter case might be an add-on economic evaluation comparing G-CSF (filgrastim) versus placebo in the treatment of febrile neutropenic episodes. The economically relevant question is not only whether G-CSF decreases length of hospital stay given a set of criteria for discharge, i.e. if G-CSF increases efficiency within this context, but also what is the (cancer-specific) cost-effectiveness of the overall treatment protocol to which G-CSF is added. The same question will often apply to trials on salvage therapy.

Economic evaluation prefers the inclusion of preventive alternatives when considering diseases with a strong risk factor component (e.g. smoking in cancer of the lung, oesophagus and bladder). What seems efficient from a narrow perspective, may have an unfavourable cost-effectiveness from a broader view.

\subsubsection{Choice of end points and follow-up time}

This issue pertains both to the principal question of which outcome measure is at stake, and to the practical question of which end point results in a feasible sample size, given a reasonable time frame and the usual values for precision and power. Theoretically, the primary outcome measures in economic evaluations are cumulative life years gained and differential costs. The usual primary end points in cancer trials are fixed time (e.g. 1 year or 5 years) survival rates and disease-free survival rates. From a clinical viewpoint, the 
choice of the analytical follow-up time (for example, 1 year or 5 years) may be difficult if the intervention has lifelong influence on survival (e.g. breast cancer) or if the impact of alternatives on survival differs over time. Statistical methodology exists to deal with incomplete empirical follow-up (censoring of observations), enabling comparisons of survival rates. In economic evaluation, lifelong follow-up is also preferred. Except for rare cases with either complete recovery or death within the empirical follow-up time, lifelong consequences cannot be observed. What happens after censoring should be explicitly estimated, which requires advanced statistical analysis. Usually mathematical modelling is applied, based on comprehensive data of patients at the time of censoring (Bonsel et al., 1990; Van Hout et al., 1993). Systematic studies on patients with recurrent or terminal disease are rare, and cancer is no exception. Consequently, valid lifelong estimates may be difficult to obtain.

Related to the end point definition and the empirical follow-up is the determination of the sample size. From a conceptual point of view, economic evaluation requires a separate sample size determination, based on distributions of costs and benefits in any of the alternatives distinguished. In practice, lack of reference values for costs and quality of life data may restrict sample size considerations to the clinical part of the trial. However, in economic evaluation, statistical significance of differences and the absolute magnitude of differences are equally important (De Koning et al., 1991).

\subsubsection{Determination of outcome}

In economic evaluation, the determination of length of survival is straightforward, except for the modelling of incomplete observations. The measurement of health status/quality of life changes is more complex. Measurement instruments can be divided into two main categories: the generic instruments and illness-specific questionnaires. Generic instruments are developed to monitor changes in all health dimensions and allow comparison of outcomes across different disease categories. The Nottingham Health Profile, the RANDMOS 20 and the related Short-Form 36 questionnaire seem to be suitable instruments. Illness-specific instruments measure health problems specific for an illness. These instruments are more sensitive for specific problems concerning a particular disease. Cancer-specific instruments often used are the Rotterdam Symptom Checklist, the EORTC Core Quality of Life Questionnaire and the Cancer Rehabilitation Evaluation System-Short Form. So far, clinical and economic evaluations coincide. 
As explained earlier, economic evaluation also tries to arrive at a summary value of the patient benefits. The following procedure applies. First, so-called utility values (a figure between 0 and 1 to indicate the preference value of a certain health state) have to be attached to outcomes. There are three dominant methods of acquiring utility values: direct rating, standard gamble (more convenient in surgical interventions) and time trade-off (more convenient in medical interventions). Utility values may be elicited from the general public (preferable within the societal perspective) and the patients themselves. Torrance provides an extensive description of these utility techniques (Torrance, 1986). If utility values for each characteristic health state have been acquired, the construction of quality adjusted life years (QALYs) becomes feasible, allowing the improvement in outcome to be expressed in terms of QALYs gained and, consequently, the construction of a cost-utility ratio.

\subsubsection{Determination of costs}

The first step is tabulation of resources in appropriate natural units (Drummond et al., 19871), with an emphasis on the counting of variable cost items. A priori estimation of the relative importance of these cost items should guide the data collection (Turk et al., 1989). Sometimes, costs per diagnosis-related group (DRG) may be readily available (Munoz et al., 1989). Case registry forms, as normally used in CCTs, provide insufficient information as tabulation of major cost items is usually incomplete. Contrary to these forms, hospital administration data are usually complete, but they contain only a few cost items which can be related to patients. As a result, enumeration of hospital cost volumina is performed using a mixure of appended case registry forms, patient questionnaires, hospital data, and occasionally, on-site observations. Additionally, hospital administrative data provide information on fixed costs (overheads) and patient questionnaires are useful for obtaining information on direct patient's costs, indirect costs and costs related to outpatient care.

Next, prices are attached to these natural units. Frequently, prices differ substantially from the charges for a particular service (in both directions, see section on Economic Evaluation). In the last step, volumina and prices are multiplied. In addition to the empirical costs determination, economic evaluation includes estimates of future costs (disease-related and disease-unrelated) if follow-up of patients is incomplete. Particularly if the tail of the survival distribution is long - the usual case in cancer - these long-term estimates are of vital importance: average costs (as used in cost-effectiveness ratios) may largely exceed median costs (Carlsson et al., 1989). 


\subsubsection{Ethics}

In our view, economic evaluation does not carry with it specific ethical issues regarding data collection. From an ethical point of view, people may judge separately whether or not to participate in an add-on economic evaluation. So far, we have never been confronted with a refusal for the economic part of the CCT, perhaps because most patients and their families face material and immaterial costs, which they are not compensated for, but which they feel are identified by economic evaluation. More frequently, we met with some reluctance of hospital administrators and doctors to cooperate in case a substantial departure of economic cost estimates from current charges and fees was likely to be determined.

\subsubsection{Generalisation}

From an economic perspective, the generalisability of CCT results is usually rather limited due to a variety of reasons. Clinical research attempts to demonstrate validly the effectiveness of a technology and, consequently, the design (patient selection, treatment allocation, treatment guidelines) is rigorous. Moreover, research patients are more compliant, clinical investigators are more competent, and the hospital setting offers more facilities than its counterparts in general practice. The better a CCT satisfies design criteria, the more easily the study results will be accepted for their reproducibility and scientific soundness. However, these criteria and the selective environment often drive a CCT far from the average clinical practice, and thus the role of CCT in health care decision making outside the CCT environment may be limited.

For cross-country generalisation, the general level of a national health care system (quality, accessibility) requires attention, particularly in economic evaluation. What may be regarded as routine hospital care for any patient in one country, may be care which is restricted to special cases in another. Cultural differences may account for other patterns of care. For example, in the Netherlands, an important role of the general practitioner and close relatives in terminal care may be observed, whereas in other EC countries emphasis may be more on hospital care. Two additional factors limit generalisability from the economic perspective. For the same health care services, prices between and even within (Clark, 1992) countries show differences to such an extent that cost-effectiveness ratios are usually incomparable (Shapiro, 1992). Besides, the cross-cultural application of values attached to health care benefits requires more investigation. 


\subsection{Specific issues in economic evaluation in cancer}

Economic evaluation sometimes causes uneasiness in patients and doctors, and occasionally the introduction of economic considerations is even stated to be unethical. This is particularly true for disease groups with specific societal connotations like cancer and AIDS, and for the terminal stages of any disease (Hancock, 1992). We think that in view of the general scarcity of medical resources, all disease groups should be judged alike: a preterm painful death from systemic lupus or sclerosing cholangitis is not preferable to dying from cancer due to its pathophysiological base alone. The reluctance to apply economic evaluation in everyday practice may partially explain the current restriction of economic evaluation to screening programmes (breast cancer, colo-rectal cancer): less than $10 \%$ of the recent Medline literature on costs and cancer refers to non-screening topics.

A recurrent problem in the economic evaluation of cancer treatment is the complete definition of alternatives. As the perspective is lifetime, it is important that the treatment protocols include details on long-term treatment. Generally, the highest costs in cancer are incurred after its detection (incidence costs, recurrence costs), and during the terminal phase (mortality costs) (De Koning et al., 1991; Beck-Friis et al., 1991; Bried and Scheffler, 1992). Thus, in the economic evaluation of primary therapies, significant differences may arise (particularly in quality of life and costs) due to uncontrolled variations in terminal treatment. If data on terminal stages are unavailable, combinations of trial data with "adjuvant" modelling using various "average" data are required (Baker et al., 1991). Another problem encountered in cancer trials is the "minimal" change of a protocol during study. The cost impact of these changes is usually underestimated, if estimated at all, and trial data before and after the change may not be safely combined. The same argument applies to uncontrolled inter-centre variations in supportive treatment, particularly with antibiotics.

Three problems of outcome measurement deserve attention. First, the general lack of prognostic models for the more advanced stages of disease. Second, the understandable lack of knowledge on long-term effects of new life saving approaches (e.g. bone marrow transplantation (Hillner et al., 1992)). Both can only be dealt with through sensitivity analysis. Finally, the observed reluctance to apply comprehensive quality of life measurements because of the presumed burden on patients. If properly informed about the purpose and contents, few patients in our experience withdraw from completing extensive quality of life questionnaires: most of them express gratitude for the opportunity to communicate their feelings, abilities and general rating of life. 
A specific issue in cost measurement is the appropriate measurement of costs incurred to the patient (waiting, travelling) and his or her family (Beck-Friis et al., 1991; Houts et al., 1984). These are usually measured by way of a patient questionnaire, and in valuation, standardised methods are applied. Another problem to deal with is the fair assignment of costs to the diagnostic and therapeutic nucleus on the one side, and to costs which are induced by the research protocol on the other side. The latter may be considerable (Kennedy, 1991). If the CCT is multi centred, the usual case, heterogeneity between hospitals on costs may be disturbing. Volume heterogeneity is generally caused by different policies not dealt with in the protocol (e.g. antibiotic prevention and curation, rehabilitative services). Price heterogeneity may be caused by incomparability of the cost structure of cancer treatment, even within one country. Even if the potential effect of policy heterogeneity on effectiveness is neglected, cost analysis may be cumbersome.

\subsection{Discussion}

We believe that economic evaluation can play a valuable role in decisions on the adoption and utilisation of new technologies (Laupacis et al., 1992). Economic evaluation in the field of medical research may take two forms, either as an add-on study or as a primary economic study. In both cases, specific economic features concern the mode of comparison, the defined alternatives, the primary end points (effects and costs), the perspective (society), the time period (lifetime) and finally, the generalisability of results. The add-on study is the more familiar approach, and particularly in areas of clinical research with high methodological standards, as in oncology, the pros and cons of add-on economic evaluation disclose much of the underlying philosophies. In some features (comparative mode, to some extent the choice of alternatives, the perspective), the requirements of $\mathrm{CCT}$ and economic evaluation are similar. In others (costs, time period), economic evaluations require extension rather than change of a given CCT protocol, though the work load involved may exceed the clinical research part of the CCT. Good clinical practice standards are more established in hospitals than appropriate economic and administrative management, implying a major empirical task of cost measurement.

Apparently, some unresolved discrepancies remain: the width of the scope of alternatives, the role of quality of life and utility measurement, and the attitude towards generalisability. We believe that they will disappear with the further integration of evaluative methods, and also that these changes will be observed in clinical areas, like oncology, with a strong methodological tradition: the gradual change of medical services from a mythic event to a societal commodity is irreversible. 


\section{4}

Measurement and valuation of quality of life

\subsection{Preface}

The international literature on quality of life research is vast and rapidly expanding. Evaluating instruments for applications within cancer trials is a continues process. The paper presented here is based on a literature review from 1980 to 1992. More recently, information on the psychometric features of the Short Form-36 (SF-36), the European Organisation for Research and Treatment of Cancer Core Quality of life Questionnaire (EORTC QLQ-C30), and the Functional Assessment of Cancer Therapy Scale (FACTscale) has become available. In Chapter 13 paragraph 2 the use of these instruments in economic appraisal of cancer treatments will be discussed.

\subsection{Summary}

In the economic evaluation of technologies in cancer treatment at least three end points are relevant: costs, survival and quality of life. This article is focused on quality of life. Economic evaluation requires the use of generic and valuation quality of life instruments at a disease non-specific level, but the inclusion of cancer-specific instruments may be advisable, particularly for reasons of explanation if changes in dimensions are small or conflicting. Given the pros and cons of the available questionnaires, we advocate the use of the Nottingham Health Profile, the EuroQol and the Rotterdam Symptom Checklist. In our experience the quality of life issue in economic evaluation linked with cancer trials is associated with practical problems like questionnaire composition, follow-up time, interviewing schedule, patients' compliance and doctors' acceptance. These problems are discussed and some practical guidelines for the design of quality of life measurement in cancer trials are given. 


\subsection{Introduction}

Cancer is a major cause of illness and death, responsible for almost a quarter of total mortality in Western countries. During the last few decades cancer treatment has shown a rapid evolution. It is now a multidisciplinary treatment strategy incorporating surgery, radiotherapy, chemotherapy and/or immunotherapy. Treatment usually has important sideeffects, especially radiotherapy and chemotherapy. For example, radiotherapy causes tiredness, skin injury and emotional discomfort. Chemotherapy, often considered even more burdensome, is given over longer periods and its toxicity causes hair loss, nausea and vomiting, fatigue and emotional problems. Consequently, those involved in the care and treatment of cancer patients have wondered whether improvements in survival probabilities outweigh the burden of these severe side-effects in all cases (De Haes and Knippenberg, 1985). Not only life years gained, but also the quality of years alive is at issue.

The high incidence and prevalence of cancer make it a major economic issue. The direct medical costs are considerable, especially during primary treatment and metastatic periods and amount to $5 \%$ of health care expenditure in the Netherlands (Koopmanschap et al., 1991). Future expenditure may be even higher due to the increased use of expensive drugs and radiotherapy equipment, the growing emphasis on various kinds of palliative interventions and survival success. The prevalence of morbidity, mortality and the consequent loss in labour and household production also cause substantial indirect costs. These phenomena and trends imply a growing role of economic evaluation in the cancer field and justify consideration of the specific problems of applying economic evaluation in this area. In the economic evaluation of technologies in cancer treatment at least three end points are relevant: costs, survival and quality of life. Quality of life will be the focus of our discussion. The discussion is based on a literature survey and our own experience with economic evaluation linked with cancer trials in the Netherlands, concerning patients with haematological malignancies and solid tumours.

\subsection{Objectives of quality of life research}

When two treatment modalities differ with respect to quality of life the main clinical end point, mortality, should be considered in relation to outcome indicators for quality of life, such as clinical outcome data, disease-specific quality of life measures and general scales, although the choice between these is not clear cut. Drummond and Davies (1991) point out that the different types of measures complement each other by adding important 
information about the consequences of various health care interventions. The usual objective of carrying out quality of life research is to gain insight into the reactions of patients to cancer and on cancer treatment and the interrelations of these different reactions with the overall quality of life. Furthermore, data concerning the impact of a certain therapy on quality of life may increase the knowledge about the effectiveness of such therapy (De Haes and Knippenberg, 1985).

Quality of life studies generally give a description of health status. Depending on the type of study, they deal with more or less objective features of health. Most studies use dimensions of the concept such as the physical, psychological and social dimensions (Hörnquist, 1982). More extended concepts even incorporate material and religious dimensions. To evaluate a specific therapy in clinical studies, the end points most commonly used are survival rates, disease- and/or symptom-free survival, response rates and response duration. When quality of life is taken into account, there is more emphasis on the physical and psychological aspects of life, sometimes complemented with aspects of social functioning.

Economic evaluation is intended to support health policy at different levels of decision making. In cancer treatment, decisions are generally made in a public sector context, implying that one tries to maximise health outcome given a social budget. This requires the summary measure used in economic evaluation to be applicable to health care interventions in general, and also to be uniform in the sense that all interventions are to be judged in an equal manner. If the treatment is to be seen more as a private good, and subject to an individual decision, economic methods exist to derive a numeric value for the utility of outcome; thus a cost-benefit analysis becomes feasible, allowing an absolute judgement on the efficiency of a treatment strategy.

From the economic perspective, the objective of studying quality of life is to determine the contribution of changes in quality of life to a summary measure for the outcome of an intervention. Such summary measures may be in natural units (as "life years" commonly used in cost-effectiveness analysis), in terms of utility (as in cost-utility analysis) or in monetary terms (as in cost-benefit analysis). When there is more than one effect parameter (e.g. survival and health status improvement) or when effects are measured in more dimensions of quality of life, a value judgement (utility weight) should be given to each of these parameters or quality of life dimensions in order to arrive at such summary measures. As in cancer treatment, both survival and quality of life (in several dimensions) are important aspects of outcome, and only cost-utility and cost-benefit analysis are appropriate instruments to measure the efficiency of cancer treatment. 


\subsection{Description and valuation of quality of life in economic evaluation}

Descriptive instruments can be divided into two main categories: the generic instruments and disease-specific questionnaires (Patrick and Deyo, 1989). Generic instruments are developed to measure all health dimensions. They can be used over a wide range of disease categories and are, therefore, suited to function as generally applicable descriptive instruments for measuring quality of life improvement in economic evaluation. In this respect they provide a reference for quantitative assessment of quality of life changes in economic evaluation and have been used in many economic appraisals.

Generic instruments most frequently used are: the Nottingham Health Profile (NHP) (Hunt et al., 1986), the Sickness Impact Profile (SIP) (Bergner et al., 1981) and the abbreviated RAND-Medical Outcome Study questionnaire (Stewart et al., 1988). The newly developed Short Form-36 may be regarded as an extension of the abbreviated RAND-Medical Outcome Study questionnaire / RAND-MOS 20, or indeed as a reduction of the original longer RAND-MOS questionnaire (McHorney et al., 1992).

Disease-specific instruments measure health problems specific for an illness or disease category. These instruments are measuring specific quality of life aspects which are typical for a particular disease, and are, therefore, more sensitive for changes in health of patients having that disease. Concerning cancer, these instruments can be divided into:

a. Cancer-specific questionnaires (for all cancers) and

b. Specific cancer questionnaires (for one type of cancer).

The following cancer-specific instruments are often used: the Functional Living Index for Cancer (Schipper et al., 1984), Spitzer QL index (McDowell and Newell, 1987), European Organisation for Research and Treatment of Cancer Core Quality of Life Questionnaire (EORTC Core QLQ) (Aaronson et al., 1988), Cancer Rehabilitation Evaluation System-Short Form (CARES-SF) (Ganz et al., 1992) and the Rotterdam Symptom Checklist (De Haes et al., 1990). Examples of specific cancer instruments are: the Breast Cancer Chemotherapy Questionnaire (Levine et al., 1988), the Performance Parameters for Head and Neck Cancer (Lansky et al., 1988) and the Linear Analog SelfAssessment (LASA) of Voice Quality for laryngeal cancer (Llewellyn-Thomas et al., 1984).

For the description of quality of life in economic evaluation, generic instruments suffice for overall comparison and testing, but the inclusion of specific instruments may be 
advisable, particularly for reasons of explanation if changes in dimensions are small or conflicting. By using generic instruments it is not always possible to gain insight into how changes in health have taken place. For some generic questionnaires, an algorithm is available to calculate a summary measure, but this may not reflect the weights of the various dimensions in the health questionnaire as may be derived from value judgements of the relevant patient group or other relevant representative groups.

When strategies score differently on different dimensions and when one needs a summary measure of outcome comparable across a range of interventions, it is necessary to get valuation of health states in one single dimension representing an overall judgement of quality of life of a health state. This would then allow the calculation of "quality adjusted life years", where a life year gained is "adjusted" using this value judgement for quality of life. To acquire valuations, respondents are requested to value health states. These states are derived from a set of health state descriptors. The values usually range from 0 to $1(0=$ "worst imaginable health state", 1 = "best imaginable health state"). By means of modelling techniques a multi-attribute utility function may be derived to predict values of any composition of health states. There are three methods of acquiring valuations:

- direct rating (e.g. determining a point on a line with clearly defined end points),

- $\quad$ standard gamble (choosing between options under uncertainty) and

- time trade-off (choosing between options with different durations of a particular health status) (Drummond et al., 1989; Torrance, 1987).

Furthermore, there are two basic approaches used to measure the utility of health status. In one approach, actual patients who are in a particular health state are used as subjects to determine their utility for such a state. Using subjective utility assessments is most in line with conventional welfare theory. In the other approach, the health states are assessed by subjects who may or may not have personal experience with the health state being measured, but are thought to represent public opinion. Which approach is preferable depends on the viewpoint of the study. When a study is conducted from the societal viewpoint and is pertinent to public policy decision making, the appropriate utilities are those of the general public (Drummond et al., 1989). If a fixed set of utility values generated from a representative sample from the general public can be related to all possible outcomes from a generic health questionnaire, and if such a generic instrument would then be used to measure health outcomes in an economic evaluation, this would add to the comparability of results from economic evaluations and be in line with the public sector context of decision making to be supported with results from an economic evaluation. The problem with soliciting value judgements from persons without relevant 
disease experience is that the person should truly understand what the described health state is like. For some of the generic health measurement instruments such a set of utility values is known allowing the calculation of QALYs gained when such a generic instrument is used to assess health outcomes. The descriptive part of the EuroQol instrument is especially developed to be used in economic evaluations and a validated set of utility values is available for this instrument (EUROQOL Study Group, 1990; Essink-Bot $e t$ al., 1990). Other available valuation instruments are the Quality of Well-being Scale (QWB) (McDowell and Newelll, 1987), the McMaster Utility Measurement Questionnaire (MUMQ) (Bennett and Torrance, 1990), the Torrance's Health State Classification System (Torrance et al., 1982) and the Rosser \& Kind Index (Rosser and Kind, 1978).

\subsection{Choice of measurement instruments}

In clinical trials the choice of measurement instruments depends on criteria such as psychometric features (internal consistency, reliability, validity, etc.), specific patient features, feasibility and on the possibility of gaining insight into the quality of life aspects of the disease itself, and into the side-effects of treatment. Quality of life measurements in economic evaluation should foremost allow comparison across different technologies and diseases. This requires the inclusion of a generic health measurement instrument with the opportunity to derive utility values (or indeed an instrument soliciting value judgements directly from the patients) and, if deemed necessary for reasons explained above, the inclusion of a cancer-specific instrument. The precise combination of instruments depends on the required psychometric and specific patient features and feasibility aspects. An important aspect of the latter is the duration of the whole interview. Table 4.1 describes the psychometric features and the duration of the interview.

Concerning the generic instruments, there are hardly any differences between the psychometric features of the instruments mentioned. The test-retest reliability is slightly in favour of the NHP and the SIP. Concerning the internal consistency per dimension the NHP is preferable to the SIP. All instruments mentioned have content and construct validity. Considering the psychometric features, we prefer the NHP to the SIP and the RAND-MOS 20. 
4 Quality of life measurement

Table 4.1 Description and valuation of quality of life

\begin{tabular}{|c|c|c|c|c|}
\hline Measurement instrument & Reliability & $\begin{array}{r}\text { Internal } \\
\text { consistency }\end{array}$ & Validity & $\begin{array}{l}\text { Interview } \\
\text { duration }\end{array}$ \\
\hline $\begin{array}{l}\text { Generic: } \\
\text { Nottingham Health Profile } \\
\text { - Physical mobility } \\
\text { - Pain } \\
\text { - Sleep } \\
\text { - Social isolation } \\
\text { - Emotional reaction } \\
\text { - Energy } \\
\text { Sickness Impact Profile } \\
\text { - Physical } \\
\text { - Psychological } \\
\text { - Overall } \\
\text { Rand Medical Outcome } \\
\text { Study (RAND-MOS 20) } \\
\text { - Physical functioning } \\
\text { - Role functioning } \\
\text { - Bodily pain } \\
\text { - Social functioning } \\
\text { - Mental health } \\
\text { - General health perception }\end{array}$ & $\begin{array}{l}0.90-0.91 \\
0.79-0.84 \\
0.75-0.92\end{array}$ & $\begin{array}{l}0.81 \\
0.77 \\
0.69 \\
0.42 \\
0.75 \\
0.68\end{array}$ & $\begin{array}{r}\text { Content } \\
\text { Construct }\end{array}$ & $20-30 \mathrm{~min}$. \\
\hline $\begin{array}{l}\text { Cancer-specific: } \\
\text { Rotterdam Symptom } \\
\text { Checklist } \\
\text { Cancer Rehabilitation } \\
\text { Evaluation System-Short } \\
\text { Form (CARES-SF) }\end{array}$ & $\begin{array}{r}0.82-0.88 \\
0.86\end{array}$ & $\begin{array}{l}0.81-0.93 \\
0.61-0.85\end{array}$ & $\begin{array}{r}\text { Content } \\
\text { Construct } \\
\text { Concurrent }\end{array}$ & $\begin{array}{l}8 \mathrm{~min} . \\
\text { Unknown }\end{array}$ \\
\hline $\begin{array}{l}\text { Valuation: } \\
\text { Quality of Well-being } \\
\text { Scale (QWB) } \\
\text { EuroQol }\end{array}$ & $0.69-0.94$ & Unknown & $\begin{array}{r}\text { Content } \\
\text { Construct } \\
\text { Content }\end{array}$ & $\begin{array}{l}18 \text { min. (descr) } \\
\text { Unknown (val) } \\
1 \text { min. (descr) } \\
20 \text { min. (val) }\end{array}$ \\
\hline
\end{tabular}




\begin{tabular}{|l|r|r|r|r|}
\hline Measurement instrument & Reliability & $\begin{array}{r}\text { Internal } \\
\text { consistency }\end{array}$ & Validity & $\begin{array}{r}\text { Interview } \\
\text { duration }\end{array}$ \\
\hline Valuation: & & & & Content \\
$\begin{array}{l}\text { McMaster Utility } \\
\text { Measurement Questionnaire } \\
\text { (MUMQ) }\end{array}$ & $0.66-0.94$ & Not relevant & $45 \mathrm{~min}$. \\
$\begin{array}{l}\text { Torrance's Health State } \\
\text { Classification System }\end{array}$ & $0.86-0.94$ & Unknown & Unknown & Unknown \\
Rosser \& Kind Index & $0.79-1.00$ & Unknown & Content & 10 min. (descr) \\
& & 1.5 hrs (val) \\
\hline
\end{tabular}

References: De Haes, et al., 1990; Essink-Bot and Rutten-Van Mölken, 1991;

Schag et al., 1991; descr, descriptive point; val, valuation point.

The RSCL and the CARES-SF seem to be suitable cancer-specific instruments to be used in economic evaluation. Both instruments have good test-retest reliabilities. When comparing the internal consistency and validity of the tests, there is some preference for the RSCL. In addition, this instrument has the potential to add items (illness or treatmentrelated variables). By adding items concerning possible side-effects associated with a particular treatment, it is possible to gain more insight into these side-effects. For example, in a study comparing the cost-effectiveness of a treatment with a colony stimulating factor and a conventional treatment, we added some items concerning possible sideeffects associated with the colony stimulating factor (see * in Appendix A3). As there is little information on the psychometric features of the EORTC Core QoL questionnaire, this questionnaire has not been taken into account. However, this questionnaire is still used in several EORTC trials. Thus, in the near future more information about the psychometric features of this questionnaire may be expected.

As stated, in economic evaluation valuation of health states in terms of one value judgement is necessary. The valuation instruments mentioned are divided into two parts, a descriptive and a valuation part. By using the descriptive part it is possible to construct health states which can be evaluated by the general public. The valuation part mostly consists of a thermometer which enables the patient to evaluate his or her own health state. When a study is conducted from a social viewpoint the valuations of the health states by the general public have to be taken into account, but by acquiring valuations of own health states by patients the opportunity arises to compare these with the values from 
the general public. However, when a validated set of utility values is available, the use of the descriptive part may be sufficient. Concerning valuation instruments, the Torrance's Health State Classification System, and the Rosser \& Kind Index have good reliability features, but the internal consistency of these questionnaires is unknown. The Torrance's Health State Classification System is used less often and is, therefore, not appropriate. The MUMQ lasts about 45 minutes and the Rosser \& Kind Index lasts at least 1.5 hour, so these instruments are also not appropriate for economic evaluation of technologies in cancer treatment. The valuation instrument developed by the EuroQoL group (rating scale using a thermometer) has good psychometric features. The descriptive part of the EuroQol lasts about 1 minute, the valuation part about 20 minutes.

The QWB scale also has good psychometric features, but the descriptive part lasts about 18 minutes. While combining this instrument with a generic and a cancer-specific instrument, the duration of the whole interview would become too long. For that reason we prefer the use of the EuroQol to the QWB.

When using the NHP, the RSCL and the descriptive part of the EuroQol the whole interview would last about 20 minutes. We know from experience, based on 200 patient questionnaires administered at several states in the cancer treatment, that this is acceptable to patients.

\subsection{Design and practical issues}

For the design of quality of life measurement in economic evaluation of technologies in cancer treatment at least the following issues have to be taken into account: the choice of respondent, the method of data collection, the time and frequency of administering questionnaires and the analysis of data.

Quality of life questions are usually addressed to the patient. When the patient is not in a position to answer the questions, for example, due to serious health problems, the respondent could be a relative, nurse or doctor (Sprangers and Aaronson, 1992). There are several methods of data collection available, namely verbal (interview), written (questionnaires) and the personal computer. The written questionnaires are most often used and require less infrastructural arrangements. 
Ideally, the data collected should describe quality of life of patients before, during and after treatment. The choice of time scale depends on the natural history of the disease and on the timing and pattern of treatment. During intensive treatment periods it may be important to have frequent measurements of quality of life, sometimes intervals of 1 or 2 days may be desirable (Fayers and Jones, 1983). If there is a survival benefit it is important to take into account quality of life during life years gained. In this respect, it is necessary to make a distinction between curative and palliative treatment modalities. In both modalities quality of life measurement is very important, but the outcome indicators differ. In palliative treatment modalities, quality of life is the single most important outcome indicator. For curative treatment modalities, the most important indicator is the assessment of quality of life of life years gained, which is estimated by a separate observation of survival and quality of life.

There are several constraints on the choices regarding frequency and timing of quality of life assessments. If these are infrequent, then transient effects most likely during and after each treatment, may be missed. On the other hand, enquiries which are too frequent may well be too imposing on the patient, especially as this may be a time of particular stress because of the disease and its treatment. Patients' compliance is likely to depend on the way in which the assessment forms are explained to patients if self-assessment is required, the degree of encouragement given and the frequency with which the completed forms are collected and inspected; hence in a multicentre trial, patients' compliance may vary considerably from centre to centre (Fayers and Jones, 1983). Good coordination may enhance response rates and this may require one person being responsible for all aspects of processing of the interviews.

As economic evaluation generally requires lifelong follow-up, a modelling approach may be necessary to predict lifelong consequences. By specifying a simulation model or a MARKOV model defining various states characterised by health status and/or specific treatment (associated with a cost profile), the follow-up period of the actual clinical trial may be reduced. (Figure 4.1). It will be assumed that each state can be characterised by a unique quality of life. The following disease states are possible: disease-free, partial remission, relapse (symptom-free), relapse (symptoms) and terminal state. The measurement and valuation of quality of life of patients in the terminal phase of cancer may be difficult, because these patients may be "off protocol" and difficult to approach. Acceptable compliance rates require motivation of the patient, sophistication of the procedure and a permissive attitude towards the ideal interview schedule. 
Figure 4.1 Example of a modelling approach

\begin{tabular}{|c|c|c|}
\hline $\begin{array}{l}\text { Randomisation date } \\
\downarrow \\
\text { Treatment phase }\end{array}$ & Primary treatment & $\begin{array}{l}\text { - Complete remission } \\
\text { - Partial remission } \\
\text { - Relapse } \\
\text { - Secondary treatment }\end{array}$ \\
\hline $\begin{array}{l}\text { Quality of life } \\
\text { measurement }\end{array}$ & $\begin{array}{l}\uparrow \uparrow \uparrow c c c c c \\
\text { Short-term monitoring }\end{array} \rightarrow$ & $\begin{array}{l}\uparrow \quad \uparrow \quad \uparrow \quad \uparrow \uparrow \uparrow \\
\text { Long-term monitoring / modelling } \\
1 / 2 \text { year intervals }\end{array}$ \\
\hline
\end{tabular}

To analyse the data several statistical methods and computer programmes are available. The choice depends on the design and the type of data. The study protocol should include a brief description of how the data are to be analysed, including the tests which are to be used.

\subsection{Discussion}

Quality of life measurement and valuation is an essential part of economic appraisal of programmes in cancer treatment. To improve the comparability of the results of such studies and to increase the opportunities to generalise from the resulting findings it is necessary to achieve standardisation in design of quality of life research and the choice of instruments. Given that in most prospective studies a "piggyback" economic analysis is performed, meaning that the economic evaluation is added on to a clinical trial (sometimes after major decisions on design and data collection have been taken), the economic analyst finds himself in a stronger position when there is already consensus among researchers about the requirements for quality of life measurement from the economic perspective. This paper aims at contributing to consensus building in this area.

A practical problem in the selection of instruments is their availability for use in studies. There seems to be a tendency toward commercialisation and consequent restriction of usage which may hamper a free scientific discourse on psychometric properties and pros and cons of the available instruments. In our opinion there is not yet enough empirical data to decisively assess the comparative advantages of existing measurement and valuation instruments and no thresholds should be formed for producing such evidence. 
A final problem is the measurement and valuation of quality of life in the terminal phase of cancer. In prospective trials it is often difficult to organise data collection on quality of life of patients in the final stages of the disease as they may be "off protocol" or otherwise difficult to approach. It seems practical to launch a specific research programme to measure quality of life in these stages and to analyse differences across disease categories in order to produce a set of generally applicable values to be used in other studies. 


\section{Part III}

Cost of stem cell transplantations 



\section{5}

ABMT in non-Hodgkin's lymphoma and acute leukaemia

\subsection{Summary}

In a Dutch "investigative medicine" project, where autologous bone marrow transplantation (ABMT) was compared with conventional chemotherapy in non-Hodgkin's lymphoma (NHL) patients (HOVON-3 study), a cost analysis was carried out in five Dutch hospitals. By means of detailed cost registration forms, hospital information systems and interviews with haematologists, research nurses and research analysts, the costs of ABMT were estimated at US $\$ 35220^{1}$ per patient. The HOVON-3 study only concerned NHL patients. The costs of ABMT in other patient categories were almost similar to the cost in this category with the exception of acute leukaemia patients. In general the latter patients stayed longer in hospital and needed more intensive isolation care, more blood transfusions and more antibiotics. The costs of this group were estimated at US\$ 48000 per patient.

\subsection{Introduction}

In the Netherlands, little is known about the cost of ABMT. This kind of information is important for the reimbursement policy. In 1987, a cost-effectiveness analysis concerning bone marrow transplantation had been carried out (Engel et al., 1987). This study placed emphasis on allogeneic transplantations. Furthermore, a clinical trial has been carried out in patients with non-Hodgkin's lymphoma (NHL). This study compared ABMT with conventional chemotherapy (HOVON-3 study; HOVON = Dutch Working Party on Haemato-Oncology). A cost-effectiveness analysis was linked to this trial.

Contrary to the Dutch paper, the costs mentioned in this chapter are presented in US dollars, where 1 US $\$ \approx 1.8$ Dutch guilders. 
The registration of costs took place in 5 Dutch hospitals, namely the Dr. Daniel den Hoed Cancer Centre in Rotterdam and the university hospitals in Rotterdam, Utrecht, Groningen and Maastricht. Information to calculate the unit prices was collected in the centres in Rotterdam and in Utrecht.

In April 1992 the Health Executive Board asked the HOVON-group some questions about the daily practice of ABMT in the Netherlands. One of these questions concerned the costs of this treatment modality. On request of the HOVON-board, we have carried out a side-study to calculate the average costs of ABMT in the Netherlands. For this side-study, data from the HOVON-3 cost-effectiveness analysis have been used. This analysis concerned 18 completed transplantations. This was about $15 \%$ of all ABMTs in this patient group in the period 1989 - 1991 (Dutch Health Executive Board, 1993).

The HOVON-3 study only considers patients with NHL. Therefore, we have also analysed 20 patients with acute leukaemia. Based on these data sets, the average costs of ABMT in the Netherlands have been estimated.

\subsection{Methods}

A registration system has been developed to collect information on the resource utilisation. A pre-transplantation and a transplantation period were distinguished. Unit costs of all activities during these two periods were determined, reflecting the real use of resources. As far as the Dutch tariff system reflects real resource use, a unit charge was used (Rutten et al., 1993). As mentioned above, 18 ABMTs in patients with NHL and 20 ABMTs in patients with acute leukaemia have been analysed. The year of study was 1991. The most important activities and the corresponding unit prices were as follows:

Staff: The following staff was included: doctors, nurses, anaesthetic-analysts and researchanalysts. The calculation of labour costs were based on Dutch salary scales. The average cost per hour varied from US\$17.51 (nurses) to US\$ 69.44 (doctors), including a $35 \%$ increase for social security premiums.

Hospitalisation: The costs of hospital days were divided into direct and indirect costs. The direct costs concerned manpower and materials. Indirect costs were related to overheads. The costs of normal haematological care, care in a protected environment and intensive care were analysed in detail. A hospital day costs about US\$347 for normal 
haematological care, US $\$ 536$ for care in a protected environment and US $\$ 1167$ for intensive care.

Laboratory services: The output of laboratory services is measured using a point system, and with each point (unit of output) a unit price is associated. Each laboratory service corresponds to a number of points. The price of a laboratory service can be calculated by multiplying the number of points by a unit price per point. In general, the tariff is about US $\$ 0.94$ per point. However, by summing up the direct and indirect costs of a particular laboratory and by dividing these costs by the total number of produced points in that laboratory, a global indication of the unit price per point can be obtained. The prices per point varied from US $\$ 0.62$ (biochemistry and haematology services), to US $\$ 2.81$ (virology tests).

Medical procedures: For all other medical procedures, the Dutch tariff system has been used as an approximation of unit costs. The tariffs were regarded as reflecting the real use of resources (Rutten et al., 1993). The assumption was that about $60 \%$ of the ABMT patients were covered by the Sick Fund Insurance and $40 \%$ of the patients were privately insured.

\subsection{Results}

\subsubsection{Results non-Hodgkin's lymphoma group}

The ABMT period has been divided into a pre-transplantation and a transplantation period. The following results concerned 18 patients with NHL.

Pre-transplantation period: The costs of the pre-transplantation period were divided into the costs of bone marrow harvest and other costs, such as hospitalisation, visits, laboratory services, medical procedures and medication (Table 5.1). New techniques, such as bone marrow purging, have not been applied in the HOVON-3 study, therefore these costs were not included. The costs of bone marrow harvest consisted of staffing, surgery room, medical equipment, and quality control of the bone marrow. The total personnel costs amounted to about US $\$ 678$. The costs of the surgery room included other staff (mainly administration), materials and indirect costs. These costs amounted to about US\$ 500. The costs of medical equipment and the type of equipment used differed between hospitals, but were similar when aggregated. The average costs were about US $\$ 583$. The other material costs included for example aseptic bottles, filters, medium, heparin, iodine 
glasses, gloves, injections and bone marrow filter sets and amounted to about US\$222. The quality control of the bone marrow consisted of bacteriological- and stem cell cultures.

The costs of stem cell cultures were added to the bone marrow harvest. The bacteriological cultures cost about US\$167, i.e. on average 8 cultures per patient.

Table 5.1 The average costs of the pre-transplantation period (in US\$)

\begin{tabular}{|l|r|}
\hline $\begin{array}{l}\text { Pre-transplantation period } \\
(n=18)\end{array}$ & $\begin{array}{r}\text { Costs } \\
\text { (US } \$)\end{array}$ \\
\hline Bone marrow harvest & 2150 \\
Hospitalisation and visits & 1097 \\
Laboratory services & 944 \\
Medical procedures & 433 \\
Medication & 42 \\
Total & 4666 \\
\hline
\end{tabular}

Concerning the hospitalisation, the patients stayed on average 3 days in hospital on the normal haematological care ward. Furthermore, they were visited by a dentist and an ear, nose and throat (E.N.T.) specialist. The costs of laboratory services mainly consisted of auto-/alloantibodies against platelets and granulocytes and immunotyping. The medical procedures consisted of X-rays, electrocardiograms (ECGs) and bone marrow studies. The medication amounted to about US $\$ 42$. However, this amount did not contain the high-dose chemotherapy, which the HOVON-3 patients received.

Transplantation period: The 18 patients stayed on average 37.5 days in hospital (range: 29-60). They stayed on average 12.4 days on the normal haematological care ward, 21.9 in a protected environment and 3.2 days on the intensive care ward. The average costs of hospitalisation amounted to about US $\$ 19780$ (Table 5.2). The medical procedures mainly consisted of the insertion of a central venous catheter and X-rays, ECGs and computerised tomography scans (CT-scans). Furthermore, a day before the ABMT the patients received total body irradiation (TBI).

The medication consisted of cytostatics, antibiotics, drugs for selective decontamination of the digestive tract (SDD) and anti-emetics. The demanded nutrition differed between patients: some patients received bacterial free nutrition, other patients received parenteral nutrition. 
Table 5.2 The average costs of the transplantation period (in US\$)

\begin{tabular}{|l|r|}
\hline $\begin{array}{l}\text { Transplantation period } \\
(n=18)\end{array}$ & $\begin{array}{r}\text { Cost } \\
\text { (US\$) }\end{array}$ \\
\hline Hospitalisation (hotel) & 19780 \\
Laboratory services & 3350 \\
Medical procedures & 1167 \\
Medication and nutrition & 3422 \\
Blood transfusions & 1667 \\
Total & 29386 \\
\hline
\end{tabular}

The number and type of blood transfusions varied between patients and between hospitals (for example there was a difference between the number of donors per transfusion and the filtration and/or irradiation of the transfusions). The average costs were about US $\$ 1667$ per ABMT. This amount consists of 8 (filtered and/or irradiated) units of packed cells and 9 platelet transfusions, i.e. 6 donors, filtered and irradiated. The average costs of the transplantation period amounted to about US $\$ 29386$.

Average cost of $A B M T$ : Only $80 \%$ of the bone marrow harvests actually resulted in transplantation. Therefore, for one transplantation about 1.25 bone marrow harvests had been carried out. This resulted in an average cost per ABMT of about US $\$ 35220$.

\subsubsection{Results acute leukaemia group}

The costs of 20 ABMTs of patients with acute leukaemia have been analysed separately. The patients came from the university hospitals in Rotterdam and Utrecht and from the Dr. Daniel den Hoed Cancer Centre in Rotterdam. The number of days in hospital varied from 31 to 82 days; the average hospital stay was 49.3 days. The patients stayed on average 19.8 days on the normal haematological care and 29.5 days in a protected environment. The hospital costs were about US $\$ 22694$. The costs of laboratory services, medical procedures, medication (excluding the antibiotics) and nutrition depended on the number of days in hospital. Therefore, these costs were multiplied with a factor 1.31 (49.3 days / 37.5 days). The costs of laboratory services amounted to about US $\$ 4389$ and the medical procedures costed about US\$ 1528. The costs of medication (excluding antibiotics) and nutrition were about US\$ 1864. The costs of antibiotics were analysed separately and amounted to about US\$ 3967. 
These patients received more blood transfusions than the patients with NHL. Furthermore, the administration of these blood transfusions continued after hospital discharge. As these outpatient administration of transfusions belonged to the ABMT, these costs were included. During the transplantation period, the blood transfusions costed on average about US $\$ 4342$ and after hospital discharge they costed about US\$ 3358 . The total transfusions costs were about US\$7700. The average costs of ABMT in this patient group amounted to about US\$ 48000 .

\subsection{Discussion}

The HOVON-3 study only concerned patients with NHL. As the results of this study could not be generalised over all patient groups, we have carried out a side-study. The costs of the pre-transplantation and transplantation period turned out to be comparable to other patient groups, with the exception of patients with acute leukaemia. The supportive care for acute leukaemia patients was more intensive; patients stayed longer in the hospital, needed a longer stay in a protected environment and needed more antibiotics and blood transfusions. The laboratory services, medical procedures and the other medication depended on the number of days in hospital. Therefore, we analysed the costs of ABMT in patients with acute leukaemia separately.

In 1991, the average costs of ABMT amounted to US $\$ 48000$ for patients with acute leukaemia and US $\$ 35220$ for other patient groups. However, these results did not include the costs of follow-up after an ABMT, such as outpatient visits, laboratory services and re-admissions due to complications. This information is also of importance for the decision whether or not to allocate separate funds for ABMTs. 


\section{Stem cell transplantations in several patient groups}

\subsection{Summary}

In a retrospective study, we calculated the treatment costs of 63 patients, who received either autologous bone marrow transplantation (ABMT) with recombinant human granulocyte colony-stimulating factor (G-CSF) (filgrastim) $(n=13)$ or without G-CSF $(n=22)$ or alternatively, peripheral blood progenitor cell (PBPC) transplantation mobilised by G-CSF $(n=28)$. The recovery of granulocytes, platelets and reticulocytes after PBPC was markedly accelerated as compared with ABMT with or without G-CSF. The accelerated haematopoietic recovery was associated with a reduction in platelets and red blood cell transfusion requirements, with a reduction in episodes of fever and with earlier discharge from the hospital. This resulted in the average cost per treatment of the PBPC group being almost $30 \%$ lower than the treatment costs in the ABMT groups.

\subsection{Introduction}

Bone marrow transplantation, as an adjunct to very intensive chemo- and radiotherapy, has significantly improved remission rates and survival in the treatment of acute leukaemias and malignant lymphomas. However, the procedure-associated risk of $5-15 \%$ fatal complications and the adverse effect on patients' morbidity can be serious due to a pancytopenic period of 3-4 weeks (Brandt et al., 1988). Moreover, the costs of autologous as well as allogeneic bone marrow transplantation are high, and the additional burden that these treatments place on hospital budgets raises concern (Dufoir et al., 1992; Welch and Larson, 1989). It is, therefore, relevant to not only assess the additional benefits to patients of new treatment options, but also to monitor their cost implications. 
Haematopoietic growth factors make it possible to accelerate the haematopoietic recovery after an autologous bone marrow transplantation (ABMT) and thereby reduce the therapyrelated toxicity. As a result, a reduction in the initial hospitalisation and in the number of days on intravenous antibiotics was demonstrated (Nemunaitis et al., 1991). However, although the use of haematopoietic growth factors caused a shortening of the neutropenic period, the patients still had a median duration of severe neutropenia for 2 weeks, and had more than 3 weeks of thrombocytopenia (Nemunaitis et al., 1991). Therefore, additional strategies to further shorten this period are required.

In recent years, autologous transplantation of peripheral blood progenitor cells (PBPC) has attracted considerable interest because of the potential advantages in comparison with bone marrow, with respect to haematopoietic recovery (Brugger et al, 1992; Siena et al., 1989; Kessinger et al., 1989; Advani et al., 1992). PBPC might be used in conjunction with haematopoietic growth factors to support haematopoietic recovery after high-dose chemotherapy.

This study considers the costs of PBPC transplantation mobilised by G-CSF (filgrastim) in comparison with ABMT without G-CSF and ABMT with G-CSF. The results are based on a retrospective study of detailed records of 63 patients. The perspective of the study was the hospital's point of view, that is, all hospital costs associated with PBPC and ABMT were considered.

\subsection{Patients and methods}

A total of 22 patients received ABMT without G-CSF (six solid tumours and 16 malignant lymphomas), 13 patients received ABMT with G-CSF (12 solid tumours and one malignant lymphoma) and 28 patients received PBPC with G-CSF (19 solid tumours and nine malignant lymphomas) (Richel, 1992; Richel et al., 1993). The latter group included six patients, who received a second PBPC reinfusion within 4 weeks. For the patients who received two reinfusions, the average costs of the two PBPC reinfusions were taken into account, as the aim of the cost analysis was to compare the costs of PBPC with the costs of ABMT. These patients received only one PBPC mobilisation and harvest; the costs of the pre-transplantation were not averaged.

Patients' characteristics are summarised in Table 6.1. The solid tumours mainly consisted of patients with breast cancer and germ cell tumours. The high-dose chemotherapy regimen of this group consisted of CTC (cyclophosphamide, thiotepa and carboplatin). 
The regimen for patients with malignant lymphomas consisted of carmustine, cyclophosphamide, etoposide, and cytarabine (BEAC) or cyclophosphamide, carmustine and etoposide (CBV) or carmustine, etoposide, cytarabine and melphalan (BEAM). The proportion of solid tumours and malignant lymphomas was not equal across the three treatment groups. As all treatments consisted of similar high-dose chemotherapy, regardless of $\mathrm{ABMT}$ or $\mathrm{PBPC}$, the costs of these regimen were considered as identical and disregarded in our analysis.

Table 6.1 Patients' characteristics

\begin{tabular}{|l|r|r|r|}
\hline & $\begin{array}{r}\text { Control group } \\
\text { ABMT }\end{array}$ & $\begin{array}{r}\text { ABMT }+ \\
\text { G-CSF } \\
\text { group }\end{array}$ & $\begin{array}{r}\text { PBPC + } \\
\text { G-CSF } \\
\text { group }\end{array}$ \\
\hline Number of patients & 22 & 13 & $28\left(34^{*}\right)$ \\
Median age, years (range) & $30(21-54)$ & $32(18-44)$ & $41(18-60)$ \\
Sex: & & & \\
- Male & 11 & 7 & 12 \\
- Female & 11 & 6 & 16 \\
Diagnosis: & & & \\
- Breast cancer & 1 & 4 & 11 \\
- Non-Hodgkin's lymphoma & 10 & 4 & 3 \\
- Morbus Hodgkin's & 6 & 1 & 6 \\
- Germ cell tumours & 5 & 4 & $6\left(11^{*}\right)$ \\
- Neuroblastoma & 0 & 0 & $1\left(2^{*}\right)$ \\
- Medulloblastoma & 0 & 0 & 1 \\
\hline
\end{tabular}

- Six patients received 2 PBPC reinfusions: five patients with germ cell tumours and one patient with neuroblastoma.

The PBPC mobilisation was performed on an outpatient setting. The lymphoma and germ cell tumour patients received one course of chemotherapy, namely cyclophosphamide ( 1 $\left.\mathrm{g} / \mathrm{m}^{2}\right)$ on day 1 and etoposide $\left(100 \mathrm{mg} / \mathrm{m}^{2}\right)$ on days $1-3$ intravenously. The breast cancer patients received one course of FEC $\left(500 \mathrm{mg} / \mathrm{m}^{2} \quad 5\right.$-fluorouracil, $120 \mathrm{mg} / \mathrm{m}^{2}$ epidoxorubicin and $500 \mathrm{mg} / \mathrm{m}^{2}$ cyclophosphamide). The chemotherapy was immediately followed by the administration of G-CSF at a dosage of $300 \mu \mathrm{g}$ subcutaneously, daily for 10 days.

The leucaphereses were performed with a leucapherese machine (the CS300 of Cobe) on 3 consecutive days. Both the leucaphereses and the bone marrow were cryopreserved. Additionally, a Hickman catheter was inserted. Three days after the administration of the 
high-dose chemotherapy, the stem cells were reinfused. The patients received $300 \mu \mathrm{g} \mathrm{G}-$ CSF daily until the granulocytes' recovery was stable.

The costs were considered separately for the pre-transplantation and the transplantation period. The pre-transplantation period included bone marrow (BM) or peripheral stem cell (PSC) harvest, cryopreservation and cultures, diagnostics, laboratories, hospitalisation, day-care department, medication, blood transfusions and the insertion of a Hickman catheter. The costs in the transplantation period related to days in hospital, laboratory services, diagnostics (mainly X-rays and ECGs), medication (antibiotics, anti-emetics, etc.) and blood transfusions. These costs have been registered through cost registry forms. Cost price studies have been carried out for all costs. The years of study were 1990-1992.

Statistical methods included Kruskal-Wallis tests for comparisons of costs and for comparisons of time-to-event outcomes for the days in hospital (Glantz, 1989).

\subsection{Unit prices}

For each of the activities mentioned above, unit prices were determined reflecting the real use of resources. The year of study was 1992 ( 1 US $\$ \approx 1.8$ Dutch guilders). The unit prices are summarised in Appendix A4. The cost of hospital days and treatment in a daycare department were divided into direct and indirect costs. The direct costs concerned manpower (doctors, nurses, etc.) and materials (medical devices, supportive patient care, etc.). The indirect costs were related to overheads. The cost of hospitalisation amounted to approximately US $\$ 354$ per day for normal haematological care; the direct costs were US $\$ 238$ and the indirect costs were US\$ 116 . Staying in a protected environment cost approximately US\$552; divided into direct costs of US\$ 398 and indirect costs of US\$ 154. None of the patients was treated on an intensive care ward. A day of treatment in a day-care department cost approximately US $\$ 134$ (approximately $50 \%$ direct costs and $50 \%$ indirect costs).

The output of laboratories in the Netherlands is measured in terms of a point system, and by each point (unit of output), a cost per unit or price may be associated, which differs across types of laboratories. The prices per point varied from US $\$ 0.62$ (biochemistry and haematology laboratories) to US $\$ 2.81$ (virology tests). The price of a routine test (including $\mathrm{Hb}, \mathrm{Ht}$, leucocytes and thrombocytes) amounted to 5.75 points and cost US\$ 3.58. For all other diagnostics, the Dutch tariff system has been used as an approximation 
of unit costs (an X-ray chest about US\$ 31 and an ECG US\$22). The cost of the insertion of a Hickman catheter was US $\$ 504$ (including the costs of the catheter). The drug prices used were wholesale prices. The costs of a $300 \mu \mathrm{g}$ vial of G-CSF (intravenous) amounted to US\$138.

An additional study was performed to assess the average costs of both pre-transplantation periods. The cost of BM harvest, cryopreservation and cultures was estimated at US\$ 2043. In the PBPC group, the cost amounted to US\$2740. The BM harvest was carried out on an inpatient basis, while the PCS harvest took place on an outpatient basis. The total costs of the pre-transplantation period amounted to US\$5006 for both ABMT groups, and to approximately US $\$ 5915$ in the PBPC group.

\section{$6.5 \quad$ Results}

\subsubsection{Clinical results}

Table 6.2 shows that haematological recovery was significantly accelerated with PBPC transplantation as compared with ABMT. The time to recovery of granulocytes to $>$ $0.5 \times 10^{9} / 1$ was 15.0 days for the control group, 12.5 for the patients who received ABMT with G-CSF and 8.0 for the PBPC group. For reticulocytes recovery to $>10 \%$, it was 23.0 days in the control group, 25.5 in the group receiving ABMT with G-CSF and 13.5 in the PBPC group. The median number of days for platelets recovery to $>20 \times 10^{9} / 1$ in the PBPC group was approximately half that of the control group and the ABMT with GCSF group (10.9 versus 20.2 and 23.8 days, respectively). The median number of days for temperature recovery to $<38^{\circ} \mathrm{C}$ was also lower in the PBPC group: 2.5 versus 4.6 and 5.5 days in the ABMT with and without G-CSF, respectively.

Table 6.2 Clinical results

\begin{tabular}{|l|r|r|r|}
\hline & $\begin{array}{r}\text { Control group } \\
\text { ABMT }\end{array}$ & ABMT + & PBPC + \\
& $(n=22)$ & G-CSF \\
group & G-CSF \\
& $(n=13)$ & $\begin{array}{r}\text { group } \\
(n=34)\end{array}$ \\
& (median, range) & $\begin{array}{r}\text { (median, range) } \\
\text { (median, range) }\end{array}$ \\
\hline Days granulocytes $>0.5 \times 10^{9} / 1$ & $15.0(11-17)$ & $12.5(9-18)$ & $8.0(6-11)$ \\
Days reticulocytes $>10 \%$ & $23.0(17-28)$ & $25.5(15-38)$ & $13.5(10-22)$ \\
Days thrombocytes $>20 \times 10^{\circ} / 1$ & $20.2(15-40)$ & $23.8(12-38)$ & $10.9(7-25)$ \\
Days temperature $>38^{\circ} \mathrm{C}$ & $5.5(5-12)$ & $4.6(0-12)$ & $2.5(0-12)$ \\
\hline
\end{tabular}




\subsubsection{Results cost analysis}

Patients treated only with ABMT or with ABMT and G-CSF had a mean hospital stay of 37.9 days (range 19-70) and 36.2 days (range 24-68), respectively, while patients in the PBPC group had a mean stay in hospital of 22.4 days (range 17-38) (Figure 6.1).

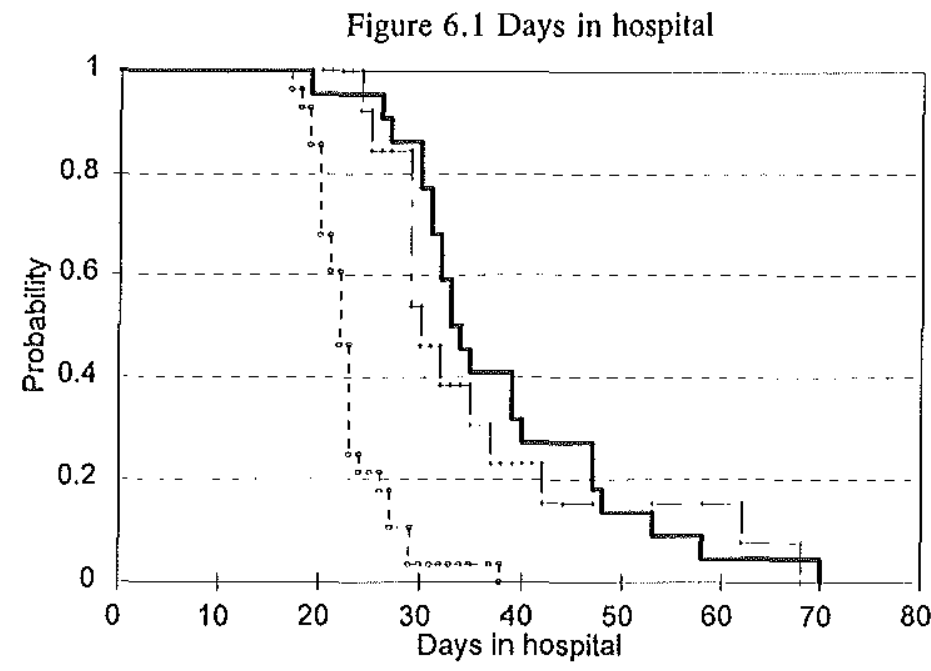

\section{- Control group -. ABMT + G-CSF .. PBPC group}

The most important cost items are summarised in Table 6.3. The patients in the control group stayed for 21 days in a protected environment, the ABMT with G-CSF group 14 days and the PBPC group only 7 days.

Table 6.3 Number of most important cost items

\begin{tabular}{|c|c|c|c|}
\hline & $\begin{array}{r}\text { Control group } \\
\text { ABMT } \\
(n=22) \\
\text { (mean, range) }\end{array}$ & $\begin{array}{r}\text { ABMT }+ \\
\text { G-CSF } \\
\text { group } \\
(n=13) \\
\text { (mean, range) }\end{array}$ & $\begin{array}{r}\text { PBPC + } \\
\text { G-CSF } \\
\text { group } \\
(n=28) \\
\text { (mean, range) }\end{array}$ \\
\hline Number of days in hospital & $37.9(19-70)$ & $36.2(24-68)$ & $22.4(17-38)$ \\
\hline Number of platelet transfusions & $47.1(18-179)$ & $70.8(10-161)$ & $26.7(9-100)$ \\
\hline Number of red cell transfusions & $10.1 \quad(3-21)$ & $13.5 \quad(4-35)$ & $6.2(1-29)$ \\
\hline Days of G-CSF treatment & - & $19.4(10-30)$ & $13.6(8-45)$ \\
\hline
\end{tabular}


The accelerated haematopoietic recovery in the PBPC group was associated with a reduction in platelets and red blood cell transfusion requirements. G-CSF was given for 19.4 days (range: 10-30) in the ABMT with G-CSF group, and for 13.6 days (range: 845) in the PBPC group.

The costs of the three treatment groups are summarised in Table 6.4. The differences across the groups in the cost of the pre-transplantation period were in favour of the ABMT groups; US $\$ 5006$ for both ABMT groups versus US $\$ 5915$ for the PBPC group. However, the differences in the transplantation period were more pronounced and definitely in favour of the PBPC group. The hospitalisation costs amounted to approximately US\$17578 for the ABMT group, US\$15603 for the ABMT group with G-CSF, and US\$ 9318 for the PBPC group.

Considering only costs, ABMT with G-CSF does not seem to be a better treatment option than ABMT alone. Although the costs of hospitalisation were lower due to a shorter stay in hospital and in the protected environment, the costs of laboratory services, diagnostics, medication/nutrition and transfusions were higher.

As compared with the other treatment groups, expenditure of the PBPC group was lower for all activities. The costs of hospitalisation decreased by more than $40 \%$. The costs of medication/nutrition and transfusions were also significantly lower when compared to both ABMT groups, as were the costs of laboratory services and diagnostics.

The costs of G-CSF were zero in the control group, US $\$ 2703$ in the ABMT with G-CSF group, and US\$ 1843 in the PBPC group. The costs of antibiotics and other medication decreased from US\$3562 and US\$3593, respectively, in the ABMT groups with and without G-CSF, to US\$1590 in the PBPC group. Combining the costs of G-CSF with medication and nutrition, the costs of medication were somewhat lower in the PBPC group (US\$ 3433 ) as compared to the control group, but this difference was not statistically significant. The medication/nutrition costs in the ABMT with G-CSF were remarkably higher (US\$6296) as compared with the other two groups.

The costs of transfusions decreased by more than $45 \%$; from US\$ 1584 and US\$2209, respectively, in the ABMT groups to US\$ 850 in the PBPC group.

The total costs in the transplantation period decreased by more than $35 \%$ in the PBPC group as compared with the other two groups. 
Although the costs of the pre-transplantation period were higher in the PBPC group than in the ABMT groups, the total treatment costs decreased significantly; from US $\$ 30592$ in the control group and US\$32443 in the ABMT with G-CSF group to US\$21809 in the PBPC group.

Table 6.4 The average costs of ABMT with and without G-CSF in comparison with PBPC transplantation (in US\$)

\begin{tabular}{|l|r|r|r|}
\hline & $\begin{array}{r}\text { Control group } \\
\text { ABMT } \\
(n=22)\end{array}$ & $\begin{array}{r}\text { ABMT }+ \text { G-CSF } \\
\text { group } \\
(n=13)\end{array}$ & $\begin{array}{r}\text { PBPC + G-CSF } \\
\text { group } \\
(n=28)\end{array}$ \\
\hline Pre-transplantation & & & \\
BM or PSC harvest, cryo- & & & \\
preservation and cultures & 2043 & 2043 & 2740 \\
Diagnostics, laboratories & 885 & 885 & 875 \\
Hospitalisation/outpatient visits & 1367 & 1367 & 402 \\
Medication/transfusions & 191 & 191 & 1378 \\
Hickman catheter & 520 & 520 & 520 \\
Total pre-transplantation & 5006 & 5006 & 5915 \\
& & & \\
Transplantation & & & \\
Hospitalisation & 17578 & 15603 & $3318^{\dagger}$ \\
Laboratory & 2626 & 3006 & $2123^{*}$ \\
Diagnostics & 236 & 323 & $170^{*}$ \\
Medication/Nutrition & 3562 & 3593 & $1590^{\dagger}$ \\
G-CSF (filgrastim) & $00^{\dagger}$ & 2703 & 1843 \\
Transfusions & 1584 & 2209 & $850^{\circ}$ \\
Total transplantation & 25586 & 27437 & $15894^{\dagger}$ \\
Total treatment costs & 30592 & 32443 & 21809 \\
\hline
\end{tabular}

Kruskal-Wallis test: ${ }^{*} P<0.05 ;{ }^{\dagger} P<0.01 ; 1$ US $\$=1.8$ Dutch guilders.

A sensitivity analysis was performed to assess the effect of changes in the costs of hospital stay, transfusions and G-CSF. The dominance of PBPC was robust, even if the savings in hospital days were not taken into account. 


\subsection{Discussion}

The aim of our study was to assess the costs of PBPC in comparison with ABMT. Previous studies have already demonstrated the feasibility of PBPC (Brugger et al., 1992; Siena et al., 1989; Kessinger et al., 1989; Advani et al., 1992; Richel, 1992; Richel et al., 1993). Our results confirm that PBPC is an effective alternative to ABMT. ABMT is a costly procedure, as specialised care units and extensive supportive care are required. A reduction in costs of ABMT through the administration of haematopoietic growth factors could not be demonstrated in our study. Our results show a reduction of hospital days, but the costs of medication, due to the additional costs of the G-CSF, and transfusions were still higher in the ABMT with G-CSF group as compared with ABMT alone. However, for almost all activities, PBPC was cheaper than ABMT with or without GCSF.

Often new treatment options show additional benefits to patients, but also add to the health care bill. To say that this will not be true for PBPC transplantation would be a premature statement as one may expect this new option, which constitutes a lighter burden both to the patient and to the financial manager in the hospital, to diffuse more rapidly in cancer treatment than ABMT. Our results suggest, however, that for patients with malignant lymphomas or solid tumours, who receive high-dose chemotherapy, PBPC transplantation is more cost-effective than ABMT. Further research will be needed to confirm this result. Moreover, as ABMT is associated with high mortality and morbidity rates, it may be worth also taking the effectiveness, including patients' quality of life, of PBPC into account. A "piggyback" economic analysis (an economic evaluation performed alongside a clinical trial) may be combined with future prospective trials to confirm the dominance of PBPC over ABMT in the patient groups considered here. 
II Cost of SCTs 


\section{Stem cell transplantation in patients with multiple myeloma}

\subsection{Summary}

In a retrospective study, we calculated the treatment costs of 26 patients, who received either high-dose melphalan combined with granulocyte colony-stimulating factor (G-CSF; filgrastim) $(n=7)$ or without G-CSF $(n=11)$ or alternatively, peripheral blood progenitor cell reinfusion (PBPC) mobilised by G-CSF following high-dose melphalan. In comparison with the control group, a shortening of the pancytopenic period and platelet recovery was noticed in the PBPC group. This resulted in a reduction in hospital costs, diagnostics, laboratory services, total parenteral nutrition and transfusions. The average costs per treatment in the PBPC group amounted to about US\$ 17908 as compared to US\$ 32223 in the control group, implying a cost reduction of $44 \%$ when changing to PBPC reinfusion.

\subsection{Introduction}

High-dose melphalan is associated with high response rates in multiple myeloma, but the treatment associated morbidity and mortality is high (about $20 \%$, due to complications of prolonged granulocytopenia) (Lokhorst et al., 1992; Selby et al., 1987). Transplantations with stem cells derived either from bone marrow or from peripheral blood, and/or the administration of colony stimulating factors are performed to hasten granulocyte recovery (Barlogie et al., 1990; Kessinger et al., 1989). Peripheral blood progenitor cell (PBPC) transplantation is increasingly used in the treatment of malignancies to alleviate bone marrow toxicity resulting from high-dose chemotherapy. It is introduced as an alternative to autologous bone marrow transplantation (ABMT) and has several advantages over ABMT, such as avoiding anaesthesia. It seems that infusion of PBPC after high-dose chemotherapy is associated with markedly accelerated platelet and neutrophil recovery as compared to ABMT (Kessinger et al., 1989; Advani et al., 1992; Siena et al., 1989). 
This study focuses on the costs associated with the treatment of multiple myeloma patients. The results are based on a retrospective study of detailed records of 26 patients. The treatment modalities were high-dose melphalan combined with or without the granulocyte colony-stimulating factor (G-CSF; filgrastim), or G-CSF mobilised by PBPC transplantation following high-dose melphalan.

\subsection{Patients and methods}

The study included 26 patients with multiple myeloma. The PBPC group consisted of 8 patients. PBPC were mobilised by G-CSF subcutaneously, and subsequently 1 litre of whole blood was collected by phlebotomy, kept unprocessed at room temperature and reinfused 24 hours after high-dose melphalan (Ossenkoppele et al., 1994). These patients were compared with a group of 18 patients with multiple myeloma who met the same criteria and whose pretreatment characteristics were equivalent. This group consisted of 11 patients who received no G-CSF, 4 patients who received G-CSF starting at day 28, and 3 patients starting at day 3 after chemotherapy. Despite the addition of G-CSF to 7 patients, there was no difference with regard to neutrophil and platelet recovery, days with fever, number of transfusions or hospital stay between these 7 patients and the other 11 patients in the control group.

The costs that were studied concerned days in hospital, laboratory services, diagnostics (mainly X-rays), the insertion of a subclavian catheter, medication (antibiotics, etc.) and blood transfusions. Data on these costs were collected through cost registry forms.

Statistical methods included Wilcoxon tests for comparisons of costs and for comparisons of time-to-event outcomes for the days in hospital (Glantz, 1989).

\subsection{Unit prices}

For each of the activities mentioned above, unit prices were determined reflecting the real use of resources. The costs of hospital days were divided into direct and indirect costs. The direct costs concerned manpower (doctors, nurses, etc.) and materials (medical services, supportive patient care, etc.). The indirect costs related to overheads. The costs of hospitalisation amounted to approximately US $\$ 350$ per day for normal haematological care, and US\$ 1165 for intensive care. None of the patients were treated in a protected environment. 
The output of laboratories in the Netherlands is measured in terms of a point system, and by each point (unit of output) a cost per unit or price may be associated, which differs across types of laboratories. The prices per point varied from US $\$ 0.62$ (biochemistry and haematology laboratories) to US $\$ 2.81$ (virology tests), the price of a routine test, (including haemoglobin, haematocrit, leucocyte and thrombocyte levels) amounted to 5.75 points and cost US\$3.58.

For all other diagnostics, the Dutch tariff system has been used as an approximation of unit costs (a chest X-ray approximately US $\$ 31.00$ and an electrocardiogram US $\$ 22.00$ ). The costs of the insertion of a subclavian catheter were US $\$ 504.00$ (including the costs of a catheter). The drug prices used were wholesale prices. The costs of $300 \mathrm{mg}$ melphalan (intravenous) amounted to US\$289.00 and the costs of a $300 \mu \mathrm{g}$ vial of GCSF (intravenous) to US\$138.00. (See Appendix A4 for a list of unit prices used in this study).

\subsection{Results}

\subsubsection{Clinical results}

For the 8 patients treated with high-dose melphalan and reinfusion of PBPC, the median time to recovery of granulocytes to $0.5 \times 10^{9} / 1$ was 12.5 days, for recovery of granulocytes to $1.0 \times 10^{9} / 1$ was 14 days, and for platelet recovery to $20 \times 10^{9} / 1$ was 23.5 days (Table 7.1 ).

Table 7.1 Clinical results

\begin{tabular}{|c|c|c|}
\hline Clinical data & $\begin{array}{r}\text { PBPC group } \\
\text { Melphalan }+ \\
\text { G-CSF + PBPC }\end{array}$ & $\begin{array}{r}\text { Control group } \\
\text { Melphalan } \pm \\
\text { G-CSF }\end{array}$ \\
\hline Days granulocytes $>0.5 \times 10^{9} / 1$ & $12.5(10-35)^{\circ}$ & $38.0(24-65)$ \\
\hline Days granulocytes $>1.0 \times 10^{9} / 1$ & $14(12-55)^{\circ}$ & $42(26-72)$ \\
\hline Days platelets $>20 \times 10^{9} / 1$ & $23.5(14-115)^{\dagger}$ & $36.0(25-172)$ \\
\hline Hospital stay in days & $19(16-39)^{\circ}$ & $43(33-71)$ \\
\hline Days of antibiotic use & $8 \quad(0-37)^{*}$ & $21 \quad(0-48)$ \\
\hline Number of platelet transfusions & $21(12-102)^{\dagger}$ & $54(30-228)$ \\
\hline Number of red cell transfusions & $6 \quad(3-21)$ & $(3-66)$ \\
\hline
\end{tabular}

Values are expressed as median (range); ${ }^{\circ} P<0.01 ;^{\dagger} P<0.05$. 
In comparison with the control group, the pancytopenic period and period to platelet recovery were significantly shortened. There was 1 patient whose results differed from the control group. This patient had received half body irradiation as second line therapy. G-CSF was well tolerated and no adverse effects were observed. Only 1 patient developed fever after reinfusion.

The patients in the PBPC group had a median hospital stay of 19 days as compared with 43 days in the control group $(P<0.0001)$ (Figure 7.1). Only 1 patient was treated in the intensive care department. This patient belonged to the control group and stayed there for 6 days.

Figure 7.1 Days in hospital

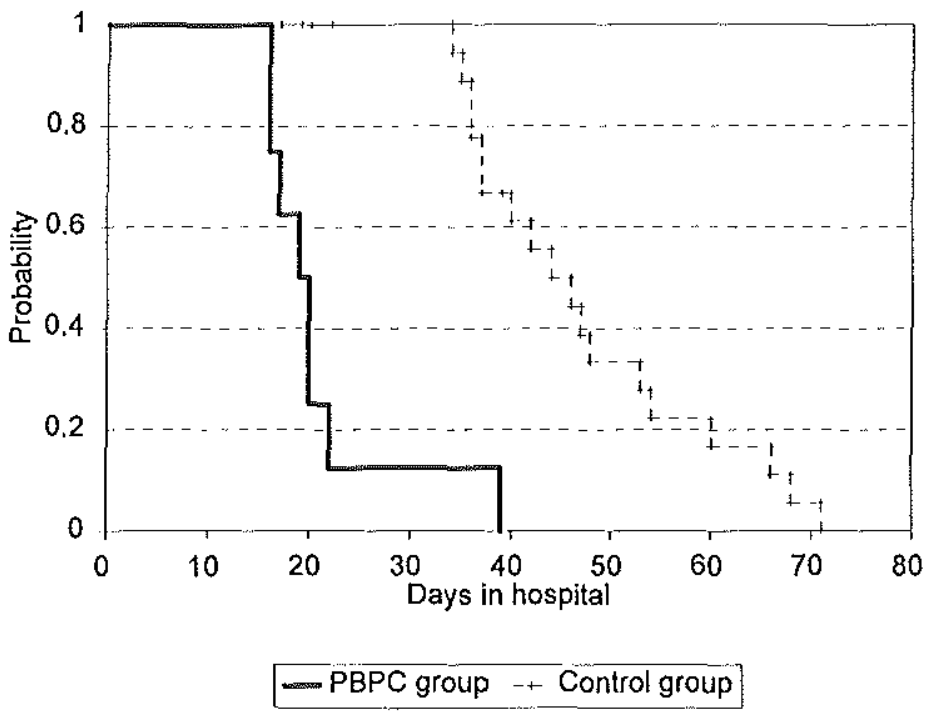

The number of days of antibiotic use decreased from 21 to 8 days. Furthermore, the number of platelet transfusions was reduced significantly from 54 to 21 transfusions. The number of red cell transfusions also decreased, but the reduction was not significant.

\subsubsection{Results cost analysis}

Table 7.2 presents the average treatment costs per category of costs. The costs of hospitalisation decreased by more than $50 \%$, from US $\$ 16747$ to US $\$ 7335(P<0.005)$. The costs of laboratory services, antibiotics and other medication, nutrition and transfusions were also significantly lower in the PBPC group. 
Table 7.2 The average treatment costs of multiple myeloma patients (in US\$)

\begin{tabular}{|l|r|r|}
\hline & PBPC group & Control group \\
& Melphalan & Melphalan \\
& \pm G-CSF \\
\hline Hospitalisation (hotel) & G-CSF + PBPC & 16747 \\
Laboratory services & $7335^{\circ}$ & 1776 \\
Diagnostics & $767^{\circ}$ & 921 \\
G-CSF (filgrastim) & $765^{\circ}$ \\
Antibiotics and other medication & 5293 & $1393^{*}$ \\
Nutrition & $2454^{\circ}$ & 6476 \\
Transfusions & $229^{*}$ & 2148 \\
Total treatment costs & $1065^{\dagger}$ & 2762 \\
\hline
\end{tabular}

${ }^{*} P<0.01 ;{ }^{\dagger} P<0.05 ; 1$ US\$ $\approx 1.8$ Dutch guilders.

The costs of G-CSF were relatively low in the control group, because not all patients received G-CSF. The costs of antibiotics and other medication reduced markedly, from US $\$ 6476$ to US $\$ 2454(P<0.01)$. When the costs of G-CSF were combined with all other medications, the costs in the study group were somewhat lower (US $\$ 7747$ versus US\$ 7869), but this difference was not significant. Only 3 patients in the PBPC group received total parenteral nutrition, while in the control group all patients received parenteral nutrition. The costs of nutrition decreased from US\$2148 to US\$229 $(P<0.001)$. The costs of blood transfusions were reduced by more than $60 \%$, from US\$ 2762 to US $\$ 1065(P<0,05)$. The total treatment costs decreased by $44 \%(P<0.005)$. The hospitalisation costs in the PBPC group were responsible for $41 \%$ of the total costs, and for almost $52 \%$ in the control group.

\subsection{Discussion}

The clinical results of PBPC reinfusion mobilised by G-CSF collected by simple phlebotomy without further processing showed a remarkable shortening of the haematopoietic recovery period in patients with multiple myeloma treated with high-dose melphalan. The neutropenic recovery was shortened with a median of 25.5 days, and the platelet recovery was also accelerated. This resulted in a remarkable decrease in hospital days and reduced use of antibiotics, platelet and red cell transfusions. One may argue that the addition of G-CSF to all patients in the control group would result in a cost reduction. However, the addition of G-CSF did not result in a reduction in the number of transfusions or in the 
length of hospital stay in the patient group studied. Therefore, we do not expect a decrease in the total costs in the control group when G-CSF is provided to all patients.

In general, when new treatment modalities become available, the additional financial burden that these treatments place on the health care system raises concern. However, in this study, PBPC reinfusion mobilised by G-CSF accounted for a remarkable reduction in costs. The reduction was observed regarding all activities in the treatment process. An improvement in the quality of life during the remission period has not been documented, but may also be expected.

PBPC reinfusion appears to be a promising approach with a favourable cost-effectiveness profile, but further research is needed to show the real benefits from the patient perspective. 


\section{Part III}

Prospective economic appraisals 



\section{8}

\section{ABMT in non-Hodgkin's lymphoma}

\subsection{Summary}

A prospective randomised clinical trial with simultaneous data collection for an economic appraisal was carried out to assess the effectiveness, quality of life and cost implications of ABMT versus standard chemotherapy in slowly responding patients with intermediateand high-grade malignant non-Hodgkin's lymphoma (NHL). The patients had a partial response after three cycles of chemotherapy and had no evidence of BM involvement of NHL. The overall and disease-free survival at 3 years were $61 \%$ and $60 \%$, respectively, in the ABMT group and $85 \%$ and $77 \%$ in the CHOP group $(P=N S)$. Moreover, there were more (severe) complaints and symptoms in the ABMT than in the CHOP group. The average costs of CHOP chemotherapy were significantly lower than the average costs in the ABMT group (CHOP: US\$ 3118 versus ABMT: US\$ 34447). Considering longterm consequences the ABMT group was more expensive (US\$ 34580 ) and patients experienced 0.14 life years and 0.22 quality adjusted life years less than the CHOP group (discount rate $5 \%$ ). As a result, changing therapy from CHOP to ABMT, as primary treatment in slow responders to CHOP, can not be recommended as the required additional investment does not produce health gains in terms of survival or quality of life.

\subsection{Introduction}

Recently, several combination chemotherapies with curative intention have been developed for patients with non-localised non-Hodgkin's lymphoma (NHL) of intermediate- and high-grade malignancy. These intensive chemotherapies, consisting of first, second and third generation regimens, have yielded high remission rates. First generation regimens, which generally include four chemotherapeutic agents, produced complete remission rates in $50 \%$ of patients and the long-term survival was about $40 \%$ (Coleman, 1985). Several 
second and third generation treatment programmes, consisting of six to eight chemotherapeutic drugs, have been developed since then (Coleman, 1985).

Initially, in these phase II single institution studies remission and survival rates improved, but prospective comparative trials did not demonstrate superiority of the new treatment programmes; the efficacy of first, second and third generation treatment regimens demonstrated similar results (Fisher et al., 1993; Gordon et al., 1992; Armitage et al., 1986). Hence, the combination of cyclophosphamide, doxorubicin, vincristine and prednisone (CHOP) has remained the standard treatment for patients with intermediateand high- grade NHL until today. For patients who do not reach a remission after the initial therapy or who relapse after $\mathrm{CR}$, the prognosis is generally poor regardless of further therapy. The most important prognostic factors in these patients seem to be whether a CR was achieved initially, as well as long duration of the remission (Armitage et al., 1986). These patients receive further treatment with combination chemotherapy. High-dose chemotherapy and ABMT is an intensive approach that may result in cure for some of these patients (Salles et al., 1994; Philips et al., 1984; Verdonck et al., 1985; Philip et al., 1987; Gribben et al., 1989), but randomised trials have not been completed to compare ABMT with standard salvage therapy in this patient group (Armitage, 1993).

In the Netherlands, a randomised, multi centre trial has been performed to analyse whether patients, who show only partial response to initial chemotherapy will benefit from early high-dose chemo-radiotherapy and ABMT (Verdonck et al., 1995). All patients were treated with CHOP and evaluated for response after three courses. Those who achieved a partial response were defined as slow responders. These patients were randomised between another 5 courses of CHOP and high-dose chemo-radiotherapy and ABMT.

ABMT is a costly procedure and most patients who undergo ABMT remain pancytopenic for 3-4 weeks, which may result in serious morbidity or even mortality (Hillner et al., 1992; Gulati and Bennet, 1992; Dufoir et al., 1992; Welch and Larson, 1989; Nemunaitis et al., 1991). Thus, a prospective economic evaluation was carried out in close conjunction with the randomised clinical trial to be able to assess the costs and benefits of CHOP therapy and $\mathrm{ABMT}$ in this patient group. In addition, we made a specific effort to monitor the quality of life of the patients. As the results of the study will be used by the Dutch Health Insurance Executive Board, we adopted a social perspective for this economic evaluation study. This perspective implies that all costs and benefits for all parties in the society will be accounted for (Bonsel et al., 1993). 


\subsection{Patients and methods}

\subsubsection{Patient population}

Untreated patients between 15 and 60 years of age with newly diagnosed NHL of intermediate- or high-grade malignancy stages II-IV (Working Formulation) were eligible (National Cancer Institute, 1982). The study group (the slow responders) consisted of 69 patients (of a total of 284 evaluable patients) who were treated in the haematology departments of eight university hospitals, one specific cancer centre and one regional hospital, participating in the Dutch Working Party on Haemato-Oncology (HOVON), between 1987 and 1993.

\subsubsection{Study design}

All patients received three courses of CHOP (cyclophosphamide $750 \mathrm{mg} / \mathrm{m}^{2}$ i.v. on day 1 , doxorubicin $50 \mathrm{mg} / \mathrm{m}^{2}$ i.v. on day 1 , vincristine (Oncovin) $1.4 \mathrm{mg} / \mathrm{m}^{2}$ i.v. on day 1 and prednisone $100 \mathrm{mg}$ per os on days 1-5). Randomisation occurred if a PR without NHL infiltration in the BM was achieved after the third course of CHOP (Figure 8.1). These patients were randomised to either ABMT preceded by high-dose cyclophosphamide ( $2 \times 60$ $\mathrm{mg} / \mathrm{kg}$ i.v.) and total body irradiation (TBI; $800 \mathrm{cGy}$; one fraction) or to another five courses of CHOP.

Figure 8.1 Study design

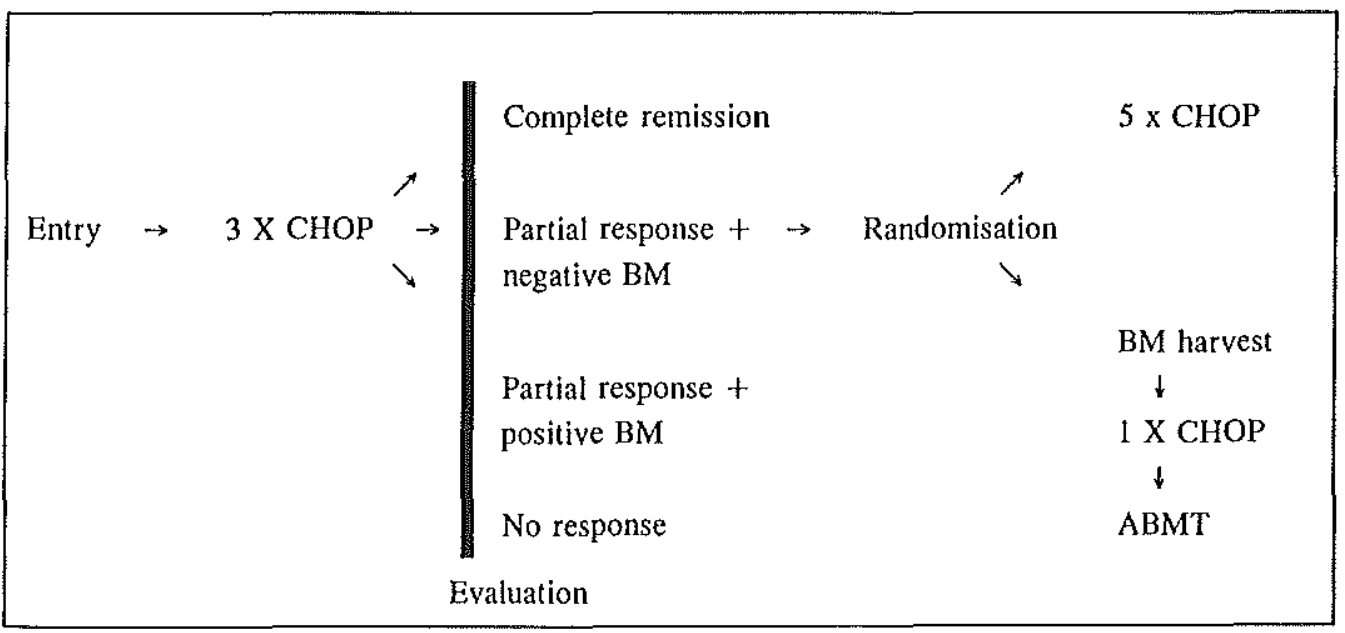


Bone marrow harvesting and subsequent cryopreservation took place as soon as complete marrow repopulation had occurred after the third CHOP course. The ABMT patients received a fourth course of CHOP the day after the bone marrow harvest. The CHOP group received five courses of chemotherapy on an outpatient basis.

\subsubsection{Effectiveness analysis}

As outcome parameters, percentage of complete remissions, the overall and disease-free survival and the quality of life after the treatment were considered. Economic evaluation requires the use of a generic health status measurement instrument at a disease-nonspecific level (Uyl-de Groot et al., 1994III). We included the Karnofsky Performance Index and the Nottingham Health Profile (Hunt et al., 1986). We also used the Rotterdam Symptom Checklist as a more sensitive instrument for measuring health status of cancer patients (Haes et al., 1990). Finally, we included the EuroQol instrument allowing the calculation of utilities (Van Hout and McDonnell, 1991). The questionnaires were administered to the patients 6 months, 1 and 2 years after the date of randomisation.

For each arm, the duration of time spent in each health state was averaged over all patients and quality adjusted life years (QALYs) were calculated by multiplying the average time spent in each health state weighted by their respective utility values and aggregating these weighted time-periods per arm (Drummond et al., 19871).

\subsubsection{Cost analysis}

The cost analysis was based on a detailed review of patient and hospital records. We examined in detail all resources used for 42 patients from four university hospitals and one cancer centre. There were 21 patients in both treatment groups. Costs considered were: days in hospital, outpatient visits, day-care departments, laboratory services, diagnostics (mainly imaging procedures), radiotherapeutical and surgical procedures, blood products, medication, parenteral nutrition and consultations.

For each of the activities mentioned above unit prices were determined reflecting the real use of resources. The year of study was 1992 (1 US $\$=$ approximately 1.8 Dutch guilders). The costs of hospital days and outpatient visits were divided into direct and indirect costs. The direct costs concerned manpower (doctors, nurses, etc.) and materials (medical devices, supportive patient care etc.). The indirect costs were related to 
overheads. The costs of hospitalisation amounted to about US $\$ 350$ per day for regular haematological care, US\$536 for staying in a protected environment and US\$1223 for an intensive care ward. An outpatient visit was costed at about US\$87 and a treatment in a day-care department at about US\$134.

The output of laboratories in the Netherlands is measured in terms of a point system and a cost price per unit may be associated with each point. The cost prices differ across types of laboratories. The cost prices per point varied from US\$0.62 (biochemistry and haematology laboratories) to US\$2.81 (virology tests). A routine test (including $\mathrm{Hb}, \mathrm{Ht}$, leucocytes and platelets) amounted to 5.75 points and was costed at US\$3.58.

The ABMT patients received total body irradiation. For the costs of this irradiation a specific cost price study was carried out (Appendix A4.2). The costs of TBI amounted to US $\$ 882$. For most diagnostics the Dutch tariff system has been used as an approximation of unit costs. The drug prices used were wholesale prices. The mean costs of one CHOP course amounted to about US\$258 (Appendix A4.1 for a list of unit prices used in this study). An additional study was performed to assess the average costs of BM harvest, cryopreservation and BM cultures. These costs were estimated at US\$2043 (Appendix A4.3).

\subsubsection{Markov model}

To estimate the lifelong consequences of the treatment modalities in terms of costs and QALYs we specified a Markov model (Bonsel et al., 1993). The following assumptions were made:

- There are three health states: complete remission (CR), no complete remission (NoCR) and death.

- The movement of patients between health states occurs every year.

- The transition probabilities for the first 3 years are derived from the clinical study.

- Patients, who are in CR for 3 years, remain in CR for the rest of their life.

- Patients who have had a CR for 2 years, but thereafter move over to the state NoCR, have a $50 \%$ chance to achieve a second $\mathrm{CR}$. When a patient did not achieve a second $\mathrm{CR}$, there is a $50 \%$ chance of dying in the next year. All other patients will die in the year after this.

- When a patient dies, it occurs on average halfway through the period remaining in a particular state. 
These assumptions were based on interviews with haematologists. As both costs and QALYs are not expected to change after 8 years, we considered a follow-up period of 8 years. Both costs and QALYs were discounted at 5\% (Drummond et al., 1987I).

\subsubsection{Statistical analysis}

Survival and disease-free survival were calculated according to the Kaplan-Meier method (Kaplan and Meier, 1959). The costs of both groups were compared for the treatment, as well as the follow-up period using the Wilcoxon test (Glantz, 1989). The quality of life items are presented as mean scores. For the comparisons between the items the MannWhitney and the Chi-square tests were used.

\subsection{Results effectiveness analysis}

\subsubsection{Treatment response}

The clinical features of the patients are shown in Table 8.1. There were no statistically significant differences in the patient characteristics (age, sex and performance status) and disease characteristics (histology, lactate dehydrogenase and stage) in both treatment groups. The median follow-up time was 36 months (range: 2-86 months).

Table 8.1 Patient characteristics

\begin{tabular}{|l|r|r|}
\hline Characteristics & ABMT group & CHOP group \\
\hline No. of patients & 34 & 35 \\
Sex: & & \\
$-\quad$ Male & 15 & 13 \\
$-\quad$ Female & 19 & 22 \\
Age: & & \\
$-\quad$ Median (yrs) & 44 & 42 \\
Tumour histology: & & \\
$-\quad$ Intermediate-grade & & \\
$-\quad$ High-grade & 25 & 10 \\
\hline
\end{tabular}


8 Cost-effectiveness of ABMT in NHL

\begin{tabular}{|c|c|c|}
\hline Characteristics & ABMT group & CHOP group \\
\hline Stage: & & \\
\hline$-\quad I I$ & 17 & 15 \\
\hline$-\quad$ III & 6 & 9 \\
\hline.$\quad I V$ & 11 & 11 \\
\hline Extranodal sites (no): & & \\
\hline-0 & 17 & 17 \\
\hline-1 & 12 & 13 \\
\hline$-\quad 2-4$ & 5 & 5 \\
\hline Lactate dehydrogenase (LDH): & & \\
\hline$-\quad<250 \mathrm{U} / \mathrm{l}$ & 7 & 7 \\
\hline$-\quad 250-750 \mathrm{U} / \mathrm{l}$ & 21 & 20 \\
\hline$-\quad>750 \mathrm{U} / \mathrm{l}$ & 6 & 8 \\
\hline Performance status (WHO): & & \\
\hline-0 & 13 & 15 \\
\hline-1 & 18 & 18 \\
\hline$-\quad 2$ & 3 & 2 \\
\hline
\end{tabular}

Following randomisation, eight patients assigned to the ABMT arm did not proceed to ABMT because of refusal $(n=3)$ and early relapse or progressive disease $(n=5)$. Of patients allocated to CHOP treatment, seven patients did not complete the eight courses of CHOP because of progression of disease. The analysis was according to the "intention to treat" approach. After randomisation, a complete remission on treatment was achieved in $68 \%$ of the ABMT patients and in $71 \%$ of the CHOP patients.

\subsubsection{Overall survival and disease-free survival}

In Table 8.2 the actuarial overall survival and disease-free survival are presented. The actuarial overall survivals at 1 year were $79 \%$ in the ABMT group and $88 \%$ in the CHOP group. At 2 and 3 years these percentages were $71 \%$ and $61 \%$, respectively, in the ABMT group and $85 \%$ in the CHOP group. The disease-free survival at 1 year was $81 \%$ in the ABMT group and $87 \%$ in the CHOP group. At 2 and 3 years these percentages 
were $68 \%$ and $60 \%$, respectively, in the ABMT group and $77 \%$ in the CHOP group. These differences were not statistically significant.

Table 8.2 Actuarial overall survival and disease-free survival at 1,2 and 3 years after randomisation

\begin{tabular}{|l|r|r|}
\hline & $\begin{array}{r}\text { ABMT group } \\
(\mathrm{p} \pm \mathrm{SE})\end{array}$ & $\begin{array}{r}\text { CHOP group } \\
(\mathrm{p} \pm \mathrm{SE})\end{array}$ \\
\hline $\begin{array}{l}\text { Actuarial overall survival } \\
\text { - at 1 year }\end{array}$ & $78.5( \pm 7.2)$ & $87.8( \pm 5.7)$ \\
- at 2 years & $71.2( \pm 8.2)$ & $84.6( \pm 6.4)$ \\
- at 3 years & $61.0( \pm 9.7)$ & $84.6( \pm 6.4)$ \\
& & \\
Actuarial disease-free survival & & $87.0( \pm 7.0)$ \\
- at 1 year & $80.5( \pm 8.8)$ & $76.7( \pm 9.2)$ \\
- at 2 years & $67.5( \pm 11.2)$ & $76.7( \pm 9.2)$ \\
- at 3 years & $60.0( \pm 12.2)$ & \\
\hline
\end{tabular}

$\mathrm{p}=$ probability; $\mathrm{SE}=$ standard error.

\subsubsection{Quality of life study}

The results are based on 51 patient questionnaires. The non-response percentage was $10 \%$.

Generic questionnaires:

The scores of the Karnofsky Performance Index were slightly in favour of the CHOP group at all measurement points in time (Table 8.3). In the ABMT group, as well as in the CHOP group, the patients were feeling better over time. Considering the first part of the Nottingham Health Profile questionnaire (NHP-I) ABMT patients at 6 months and 1 year, and the CHOP patients at 6 months reported mobility problems. The emotional reactions of the patients varied over time and between the treatment groups. At all time points both patient groups had "lack of energy". The patients in both groups had problems with sleeping in the beginning. The NHP-II deals with activities of daily life. The ABMT, as well as the CHOP treatment had consequences for work/job, home duties, sex life and interests/hobbies. 
Table 8.3 Mean quality of life scores in both treatment arms at 6 months, 1 and 2 years after randomisation

\begin{tabular}{|c|c|c|c|c|c|c|}
\hline $\begin{array}{l}\text { Treatment arm } \\
\text { Questionnaire }\end{array}$ & $\begin{array}{l}\text { ABMT } \\
6 \text { months } \\
(n=7)\end{array}$ & $\begin{array}{r}1 \mathrm{yr} \\
(n=6)\end{array}$ & $\begin{array}{r}2 \mathrm{yrs} \\
(n=7)\end{array}$ & $\begin{array}{l}\text { CHOP } \\
6 \text { months } \\
(n=6)\end{array}$ & $\begin{array}{r}1 \mathrm{yr} \\
(n=12)\end{array}$ & $\begin{array}{r}2 \mathrm{yrs} \\
(n=13)\end{array}$ \\
\hline Karnofsky Index ${ }^{I}$ & 79 & 85 & 89 & 83 & 88 & 92 \\
\hline \multicolumn{7}{|l|}{ Nottingham Health Profile $I^{2}$} \\
\hline - Mobility & 12 & 12 & 4 & 12 & 3 & 3 \\
\hline - Emotional reactions & 6 & 4 & 3 & 3 & 6 & 5 \\
\hline - Energy & 36 & 25 & 27 & 25 & 28 & 15 \\
\hline - Social isolation & 0 & 7 & 9 & 0 & 7 & 6 \\
\hline - Pain & 7 & 3 & 0 & 0 & 12 & 0 \\
\hline - Sleep & 18 & 15 & 6 & 22 & 18 & 7 \\
\hline \multirow{2}{*}{\multicolumn{7}{|c|}{$\begin{array}{l}\text { Nottingham Health Profile II } \\
\text { Present state of health causes } \\
\text { problems for (\% yes): }\end{array}$}} \\
\hline & & & & & & \\
\hline - Job or work & 29 & 40 & 29 & 50 & 33 & 23 \\
\hline - Looking after home & 43 & 17 & 29 & 33 & 25 & 15 \\
\hline - Social Life & 29 & 0 & 14 & 17 & 8 & 15 \\
\hline - Home life & 14 & .0 & 14 & 17 & 0 & 8 \\
\hline - Sex life & 29 & 17 & 14 & 67 & 8 & 15 \\
\hline - Interest and hobbies & 43 & 33 & 29 & 50 & 33 & 23 \\
\hline - Holidays & 14 & 0 & 14 & 17 & 25 & 15 \\
\hline \multicolumn{7}{|l|}{ EuroQol } \\
\hline Patient score & 54 & 81 & 87 & 78 & 76 & 88 \\
\hline Population score ${ }^{3}$ & 66 & 72 & 89 & 86 & 80 & 87 \\
\hline
\end{tabular}

'Range 0-100: from worst (0) to best health states (100); ${ }^{2}$ Range 100-0: from worst $(100)$ to best health states $(0) ;{ }^{3}$ Utility scores based on the valuations of patients and of a representative panel of the population for each health state were used to calculate average summary scores for each arm. 


\begin{tabular}{|l|lrr|rrr|}
\hline \multicolumn{1}{|c|}{ Treatment arm } & $\begin{array}{l}\text { ABMT } \\
6 \text { months } \\
(n=7)\end{array}$ & $\begin{array}{r}1 \mathrm{yr} \\
(n=6)\end{array}$ & $\begin{array}{r}\text { 2 yrs } \\
(n=7)\end{array}$ & $\begin{array}{r}\text { 6 months } \\
(n=6)\end{array}$ & $\begin{array}{r}1 \mathrm{yr} \\
(n=12)\end{array}$ & $\begin{array}{r}2 \mathrm{yrs} \\
(n=13)\end{array}$ \\
\hline Questionnaire & & & & & & \\
R'dam Symptom Checklist & & & & & \\
1. Dry mouth & 3.4 & 2.5 & 2.0 & 1.3 & 1.2 & 1.4 \\
2. Tiredness & 2.7 & 2.2 & 2.1 & 2.2 & 2.2 & 1.7 \\
3. Low back pain & 2.1 & 1.8 & 1.7 & 1.5 & 1.7 & 1.4 \\
4. Anxiety & 2.0 & 1.8 & 1.7 & 1.5 & 1.5 & 1.5 \\
5. Sore muscles & 2.0 & 2.5 & 1.7 & 1.2 & 1.8 & 1.4 \\
6. Lack of appetite & 2.0 & 1.3 & 1.3 & 1.2 & 1.2 & 1.1 \\
7. Lack of energy & 2.0 & 1.7 & 1.6 & 1.5 & 1.6 & 1.5 \\
8. Worrying & 2.0 & 1.7 & 1.6 & 1.5 & 1.5 & 1.5 \\
9. Decreased sexual interest & 1.9 & 2.3 & 1.8 & 1.8 & 1.7 & 1.4 \\
10. Rash & 1.1 & 1.2 & 1.2 & 2.0 & 1.8 & 1.3 \\
\hline
\end{tabular}

${ }^{4}$ Answer possibilities: $1=$ not at all, $2=$ a little, $3=$ quite a bit and $4=$ very much. The average values are presented.

\section{EuroQol:}

At 6 months, the patient score and the population score were highest in the CHOP group. In both groups, the patients were feeling better over time. Two years after the randomisation the score in both groups rose to 90 (average for the general population is $\geq 90$ (Bonsel et al., 1992)).

\section{Rotterdam Symptom Checklist:}

There were more (severe) complaints and symptoms in the ABMT than in the CHOP arm, especially at 6 months after randomisation (Table 8.3). The most important problems in the ABMT group were dry mouth (because of TBI), tiredness, low back pain, anxiety, sore muscles, lack of appetite, lack of energy, worrying and decreased sexual interest. Half a year after randomisation the most important complaints and symptoms in the CHOP group were tiredness, anxiety, rash and decreased sexual interest. Problems decreased over time in both groups.

\subsubsection{Long-term effectiveness}

For this analysis, we only distinguished between patients who were in $\mathrm{CR}$ and patients who were not in $\mathrm{CR}$. Table 8.4 presents the utility scores as derived from the EuroQol 
questionnaire for the two treatment arms per disease stage. For the Markov model the average utility of all patients who were not in $C R$ will be used for both treatment groups. For the patients who were in CR the scores are derived from Table 8.3. The state "death" got the utility zero.

Considering a follow-up period of 8 years, the cumulative, discounted survival amounted to 4.49 life years in the ABMT group and to 5.04 in the CHOP group on the basis of predictions using the Markov approach. The quality adjusted survival was 0.49 in favour of the CHOP group.

Table 8.4 Utilities per stage and (quality adjusted) life years

\begin{tabular}{|l|r|r|}
\hline & ABMT group & CHOP group \\
\hline Utilities per stage & & \\
CR & $0.63(n=6)$ & $0.78(n=5)$ \\
-6 months & $0.84(n=5)$ & $0.81(n=11)$ \\
-1 year & $0.91(n=6)$ & $0.92(n=12)$ \\
- 2 years & & \\
NoCR & $0.50(n=1)$ & \\
- 6 months & $0.50(n=1)$ & $0.65(n=1)$ \\
- 1 year & $0.69(n=1)$ & $0.57(n=1)$ \\
- 2 years & & $0.53(n=1)$ \\
& & \\
Markov model: 8 years follow-up & 5.01 & \\
- life years & 4.49 & 5.81 \\
- life years discounted & 4.34 & 5.04 \\
- QALYs & 3.84 & 5.06 \\
- QALYs discounted & & 4.33 \\
\hline
\end{tabular}

\subsection{Results cost analysis}

\subsubsection{Costs of treatment and follow-up}

Table 8.5 presents the average costs of treatment and follow-up per category of costs. Concerning the treatment period the hospitalisation costs in the ABMT group were responsible for $60 \%$ of the total costs, and almost $10 \%$ in the CHOP group, reflecting that the ABMT was given on an inpatient and the CHOP treatment on an outpatient basis. 
The costs of laboratory services, diagnostics, medication (including the cytostatic drugs), nutrition and transfusions were also significantly lower in the CHOP group. The total treatment costs in the ABMT group amounted to US\$ 34445 (range: US\$5772 - US\$ 69065 ) and in the CHOP group US\$3118 (range: US\$1943 - US\$6453) $(\mathrm{P}<0.01)$.

Table 8.5 Treatment and follow-up costs per patient in the ABMT and CHOP group

\begin{tabular}{|c|c|c|}
\hline & $\begin{array}{r}\text { Costs } \\
\text { ABMT group } \\
\text { (US\$) } \\
(n=21)\end{array}$ & $\begin{array}{r}\text { Costs } \\
\text { CHOP group } \\
\text { (US\$) } \\
(n=21)\end{array}$ \\
\hline Costs in the treatment period & & \\
\hline BM harvest & $2042^{\circ}$ & 0 \\
\hline Hospitalisation & $20759^{*}$ & 304 \\
\hline Outpatient visits & 207 & $766^{\circ}$ \\
\hline Laboratory services & $3128^{*}$ & 165 \\
\hline Medical procedures & $2300^{\circ}$ & 530 \\
\hline Medication and nutrition & $4368^{*}$ & 1353 \\
\hline Transfusions & $1641^{\circ}$ & 0 \\
\hline Total treatment costs & $34445^{\circ}$ & 3118 \\
\hline Costs in the follow-up period & & \\
\hline BM harvest & 117 & 333 \\
\hline Hospitalisation & 6055 & 4501 \\
\hline Outpatient visits & 2099 & 1132 \\
\hline Laboratory services & 1743 & 1147 \\
\hline Medical procedures & 3628 & 3800 \\
\hline Medication and nutrition & 1688 & 1368 \\
\hline Transfusions & 507 & 155 \\
\hline Total follow-up costs & 15837 & 12436 \\
\hline
\end{tabular}

The cost analysis includes two patients in the ABMT group who did not have an ABMT. Therefore, the average cost of an ABMT was higher, namely about US $\$ 37500$. The mean hospital stay for ABMT was 38.74 days. The patients who had an ABMT stayed on average 14.74 days in a normal haematological care unit, 19.58 days in a protected environment unit and 4.42 days in an intensive care unit. 
The follow-up period in the ABMT group concerned 20 patients, one of whom died during ABMT treatment. Twelve patients were hospitalised at least once. Furthermore, one patient received a second ABMT. This patient stayed 40 days in hospital and his total costs of treatment were US $\$ 34444$. The total costs per patient in the follow-up period amounted to US\$15837 (Table 8.5).

Twenty-one CHOP patients were followed up. In the first year only one patient died. Two patients who did not achieve a CR, received an ABMT. These patients stayed 26 and 37 days in hospital and their treatment costs were US\$ 47744 and US\$60147 respectively, The total costs per patient in the follow-up period amounted to US\$ 12436 .

The total discounted costs of the ABMT group, comprising the initial treatment costs and the costs of the follow-up, amounted to US\$ 49983 and in the CHOP group US\$15285 (Table 8.6).

Table 8.6 Total costs per patient in the ABMT group versus CHOP group

\begin{tabular}{|c|c|c|}
\hline Total costs & $\begin{array}{r}\text { ABMT group } \\
(\text { US\$) } \\
(n=21)\end{array}$ & $\begin{array}{r}\text { CHOP group } \\
\text { (US\$) } \\
(n=21)\end{array}$ \\
\hline BM harvest & $2159^{*}$ & 333 \\
\hline Hospitalisation & $26814^{*}$ & 4805 \\
\hline Visits & 2306 & 1898 \\
\hline Laboratory services & $4871^{\circ}$ & 1312 \\
\hline Medical procedures & $5928^{\circ}$ & 4330 \\
\hline Medication & $6056^{*}$ & 2721 \\
\hline Transfusions & $2148^{*}$ & 155 \\
\hline Total costs & $50282^{*}$ & 15554 \\
\hline Total costs (discounted at 5\%) & $49983^{*}$ & 15285 \\
\hline Markov model: 8 years follow-up & & \\
\hline Total costs & 57102 & 20951 \\
\hline Total costs discounted & 56512 & 20397 \\
\hline
\end{tabular}

${ }^{*} \mathrm{P}<0.01 ; 1$ US $\$ \approx 1.8$ Dutch guilders. 


\subsubsection{Long-term costs}

The costs in the follow-up period differed between patients who achieved a $\mathrm{CR}$ and patients who did not achieve a $\mathrm{CR}$ (NoCR). However, there were no significant differences between ABMT and CHOP patients. The follow-up costs of patients who were disease-free during the 2-year follow-up, amounted to US\$ 6686 in the first year and US\$ 2204 in the second year after randomisation. In the CHOP group these costs amounted to US\$ 3793 in the first year and US\$ 711 in the second year.

Patients who did not achieve a $\mathrm{CR}$, received second-line treatment. The average costs of the second-line treatment in the ABMT group amounted to US\$27128 in the first year and US $\$ 8018$ in the second year of follow-up. In the CHOP group the second-line treatment costed on average US\$24584. The second year of follow-up amounted to US\$ 8167.

Considering a follow-up period of 8 years, the cumulative costs according to the Markov approach amounted to US\$56512 in the ABMT group and to US\$20397 in the CHOP group (discount rate $5 \%$ ).

\subsection{Discussion}

ABMT is now routinely used for the treatment of several haematologic malignancies. In this study we have assessed the effectiveness, quality of life and cost implications of ABMT versus standard CHOP chemotherapy in slowly responding patients with intermediate- and high-grade malignant NHL. The results of the clinical trial showed no significant differences in the rate of complete remissions, overall survival and disease-free survival. Long-term quality of life has been measured by multiple instruments, but there were no significant differences between the ABMT and the CHOP group. However, the cumulative costs in the ABMT group were significantly higher than those in the CHOP group.

Considering the costs of ABMT and the failure of this very intensive treatment to improve overall and disease-free survival and quality of life, we conclude that early application of ABMT in slowly responding patients is not indicated. However, a small benefit in favour of ABMT cannot be completely excluded, but is very unlikely (Verdonck et al., 1995). 
Although the study results were not in favour of the ABMT group, the results may still be useful. In the literature little is known about the expenses and long-term quality of life of NHL patients following ABMT. The study shows that both long-term ABMT and CHOP survivors had a relatively good quality of life. Furthermore, the cost analysis provides insight into the costs of ABMT, chemotherapy and follow-up including relapse treatment in this patient group. As the costs are presented in detail and specific cost studies have been carried out, the results may be useful to policy makers.

Generalisation of our results may be slightly limited due to the location of the trial, that is mainly haematological intensive care hospitals. Such a "selective environment" makes it impossible to generalise study results to the average clinical practice. However, ABMT will not be carried out in average clinical practice and the treatment protocols are standardised. Thus, the costs of ABMT will hardly differ between these hospitals. However, the costs of CHOP chemotherapy and follow-up may differ from the general practice.

Generalisation of the results to other countries is not straightforward as health care organisation and prices differ. Several cost studies in patients with other diseases have shown higher cost of ABMT. Hillner et al. (1992) reported a decision analysis of ABMT in metastatic breast cancer. The ABMT cost was only based on six patients and included a 3 -month cycle. The total charges were estimated at US\$87980 and the estimated cost was US $\$ 66200$ (our result: US\$34445). The average hospital stay was 40 days and comparable with our findings. The charges for pharmacy services were US\$ 26470 and were remarkably higher than our findings (US\$ 4368). Gulati and Bennet (1992) reported the results from a randomised, double-blind, phase III clinical trial comparing ABMT with and without GM-CSF in patients with Hodgkin's disease. The patients stayed on average 40.5 days in hospital and the hospital costs amounted to US\$34400. The median total ABMT charges were US\$ 62500 and were comparable with the study of Hillner. In the study of Gulati, the high ABMT costs were mainly caused by the routine use of either a BM transplant unit or an intensive care unit, regardless of whether it was necessary. The costs per hospital day were similar to those in our study (for example, a day in an intensive care unit was valued at US\$1317 (in 1989 dollars). The costs of supportive patient care, such as medication, transfusions and parenteral nutrition, were again much higher than in our study. The reason is twofold. First, in our study the patients received less supportive care. Second, the supportive care given, especially the medication, was much cheaper. In studies concerning patients with acute leukaemia, patients stay longer in the hospital and need more supportive care than NHL patients (Dufoir et al., 1992; 
Welch and Larson, 1989). Thus, the costs of ABMT in this patient group are considerably higher.

The use of haematopoietic growth factors stimulates haematopoietic recovery and may reduce the costs of ABMT through a reduction in hospital days, antibiotics and transfusion requirements (Gulati and Bennet, 1992; Nemunaitis et al., 1991; Uyl-de Groot et al. 1994II). In the study of Gulati and Bennet the cost of ABMT with GM-CSF amounted to about US $\$ 39800$, which is significantly lower than the treatment cost in the placebo group and is almost identical to our result.

PBPC transplantation is a less costly alternative to ABMT (Uyl-de Groot et al., 1994II), but both the addition of growth factors and the use of PBPC transplantation are not expected to decrease treatment costs beyond the level of those in the CHOP group.

Although ABMT as first-line therapy was not more cost-effective than CHOP therapy in non-Hodgkin's lymphoma patients who reached a partial response after three conventional cycles of chemotherapy and with BM free from NHL, this may not hold for other patient groups, such as patients who relapse from a previous $\mathrm{CR}$ after CHOP (Verdonck et al., 1992). The additional costs of introducing ABMT for these patient groups will be considerable, but the effectiveness, including quality of life, of ABMT in these patient groups has barely been assessed (Desch et al., 1992). Further studies are needed to ascertain the benefits of ABMT in these patient groups. 


\section{9}

\section{GM-CSF in elderly patients with acute myeloid leukaemia}

\subsection{Summary}

We conducted a prospective, randomised, multicentre clinical trial comparing the effects of GM-CSF as an adjunct to intensive chemotherapy in elderly patients with acute myeloid leukaemia (AML). The study was a combined study of the EORTC Leukaemia Group and the Dutch HOVON Haemato-Oncology Group and was supported by the Dutch Health Insurance Executive Board. The cost-effectiveness analysis part of the study has been conducted exclusively in the Netherlands.

Patients of 60 years or more with untreated newly diagnosed AML were entered into the phase III study. The study was open to entry from November 1990 to October 1994. The patients were randomised to either daunomycin-cytosine arabinoside (control arm) or daunomycin-cytosine arabinoside with GM-CSF (GM-CSF arm). GM-CSF was given to the second group of patients during chemotherapy as well as during the phase of bone marrow suppression post chemotherapy. The patients were treated with one, or in case of a partial response, with two remission induction cycles, and when a complete remission was attained, they received one additional course of consolidation therapy.

The primary end point was the effect of GM-CSF on the percentage of complete remissions (CRs). Survival duration, disease-free survival, the percentage of excessive toxicity, number of nights spent in hospital and frequency of admission, number of transfusions, frequency and nature of haemorrhages and infections, number of days of haematopoietic recovery, number of episodes of fever, infections, quality of life and costs were evaluated separately.

CR was achieved in $46.2 \%$ (after induction cycle I) and $57.9 \%$ (after cycle II) of the patients in the control group and in $50.0 \%$ (after the first chemotherapy cycle) and $54.9 \%$ 
(after cycle II) of the patients in the GM-CSF group $(P=\mathrm{NS})$. The duration of survival and disease-free survival at 2 years after randomisation were estimated at $26 \%$ and $19 \%$ for the control group and $23 \%$ and $11 \%$ for the GM-CSF group ( $P=\mathrm{NS}$ ).

Thus there were no apparent differences as regards the response to chemotherapy, survival or disease-free survival as the result of GM-CSF treatment. However, there were significant differences in the time of recovery of neutrophils. For neutrophils $>0.5 \times 10^{9} / 1$ the median time to recovery was estimated at $3.74 \mathrm{wks}$ in the control group and $3.62 \mathrm{wks}$ in the GM-CSF group $(P=0.003)$. The median time of recovery of neutrophils > $1.0 \times 10^{9} / 1$ was estimated at $4.36 \mathrm{wks}$ in the control group and $3.75 \mathrm{wks}$ in the GM.CSF group $(P=0.0003)$.

Furthermore, the administration of GM-CSF resulted in moderate to severe toxicities attributed to GM-CSF. The quality of life analysis showed more problems with regard to depressed mood, diarrhoea and rash/eczema. Considering the long-term quality of life there were no significant differences between the two groups. Considering a follow-up period of 2 years, the cumulative, discounted survival amounted to 0.979 life years for the control group and to 1.007 for the GM-CSF group. The quality adjusted survival was 0.800 for the control group and 0.816 for the GM-CSF group.

The average costs of the primary treatment were higher in GM-CSF treated patients than in the control group, i.e. US $\$ 40782$ and US $\$ 34465$, respectively $(P<0.01)$. The costs during the follow-up period did not differ between the two groups. They averaged about US $\$ 11000$ during the first year of follow-up and about US\$ 6000 during the second year.

The results of this randomised clinical trial indicate that daunomycin-cytosine arabinoside plus GM-CSF is not a cost-effective treatment strategy as compared with daunomycincytosine arabinoside alone.

\subsection{Introduction}

Acute myeloid leukaemia (AML) is mainly seen in elderly patients. The generally reduced tolerance of older patients to the toxicity of chemotherapy and the increased preleukaemic conditions require special treatment strategies for AML in the elderly. Only few studies have especially dealt with therapy development of AML in elderly patients. 
Complete remission (CR) rates achieved with intensive chemotherapy in older subjects have shown considerable variation among different studies. For instance, in a total of 431 patients of 60 years and older among 5 studies, the $\mathrm{CR}$ rates following anthracycline/cytarabine treatment appear quite heterogenous: $49 \% \mathrm{CR}(n=59)$ (Keating et al., 1981), 40\% ( $n=226)$ (Yates et al., 1982), 76\% CR $(n=33)$ (Foon et al., 1981), 42\% CR $(n=43)$ (Rai et al., 1981), 28\% CR $(n=40)$ (Kahn et al., 1984), and 58\% CR $(n=31)$ (Löwenberg et al., 1989). A combination of mitoxantrone and cytarabine as first-line therapy did not result in improved complete response rates (Liu Yin et al., 1991). The results from studies also indicate that the probability of attaining $C R$ declines progressively with increasing age (Keating et al., 1981; Yates et al., 1982). In a randomised phase III study in the elderly of the EORTC Leukaemia Cooperative Group the CR rate was $58 \%$ in patients of 65 years and older (Löwenberg et al., 1989). About $20 \%$ of complete responders became "long-term" disease-free survivors even with no maintenance therapy for 2 years or more. One other comparative study of full-dose and attenuated chemotherapy in 40 AML patients of 70 years and more has demonstrated that the toxicity was better tolerated when less than standard doses of anthracycline were applied to elderly patients (Yates et al., 1982). However, when chemotherapy dosage was reduced too much, treatment became inadequate and CR rates decreased significantly (Rai et al., 1981). These observations are consistent with the conclusion that the balance between the benefits and risks of intensive chemotherapy are delicate, particularly in older patients (Keating et al., 1981; Foon et al., 1981; Kahn et al., 1984).

Once a complete remission is obtained, duration of remission and survival are usually not different from those observed in younger patients. Obviously, the gain of achieving CR in aged patients should be deliberately weighed against the probability of drastically shortened survival in others. The advent of haematopoietic growth factors which on the one hand may improve the response and on the other shorten the neutrophil regeneration after chemotherapy, might be especially important in improving the outcome of the older category of AML patients.

We conducted a prospective, randomised, multicentre clinical trial comparing the effects of granulocyte-macrophage colony-stimulating factor (GM-CSF) as an adjunct to intensive chemotherapy in elderly patients with AML. The study was a combined study of the EORTC Leukaemia Group and the Dutch HOVON Haemato-Oncology Group and was supported by the Dutch Health Insurance Executive Board. The cost-effectiveness analysis part of the study has taken place in the Netherlands only. The aim of the latter analysis was to compare the costs and effectiveness of GM-CSF as an adjunct to intensive 
remission induction chemotherapy. Special attention has been given to both the short- and long-term quality of life of the patients.

\subsection{Patients and methods}

\subsubsection{Study design and treatment}

Patients of 60 years or more with untreated newly diagnosed AML were entered into the phase III study. The study was open to entry from November 1990 to October 1994. Eligible patients had to satisfy the following criteria: 1. cytological diagnosis of AML characterised by at least $30 \%$ blast cells in bone marrow smears belonging to one of the cytological subtypes of MO through M7 according to the French-American-British (FAB) classification. Patients were eligible when WHO performance was 2 or less and when no severe heart, renal, hepatic or pulmonary disease or active uncontrolled infection were present.

Patients were not eligible when they were refractory to platelet transfusion. Patients with blast crisis of chronic myelogenous leukaemia, myelodysplasia previously treated with chemotherapy or cytokines, other malignant diseases except basal cell carcinoma of the skin and stage I cervix carcinoma were ineligible.

Figure 9.1 presents the study design. The patients were randomised to either two types of remission induction treatment, i.e., daunomycin-cytosine arabinoside without GM-CSF (control group: arm I) or daunomycin-cytosine arabinoside with GM-CSF (GM-CSF group: arm II). GM-CSF administration was initiated one day prior to the start of chemotherapy and was subsequently administered during chemotherapy (in an effort to enhance the cytotoxic efficacy of the chemotherapy by priming the GM-CSF sensitive leukaemia cells) as well as during the aplastic period post chemotherapy (in an effort to accelerate haematopoietic recovery from intensive chemotherapy and thereby mitigate haematopoietic toxicity). The patients were treated with one, or in case of a partial response, with two remission induction cycles, and when a complete remission was attained, they received one additional course of consolidation therapy (arm I; without GM-CSF and arm II: with GM-CSF).

CSF 39-300 sterile powder (GM-CSF, Leucomax ${ }^{\oplus}$, Sandoz, Basle) for injection was supplied in vials as sterile lyophilised powder formulated with mannitol, human serum albumin, polyethylene glycol, and citrate/phosphate buffer. 
Figure 9.1 Study design

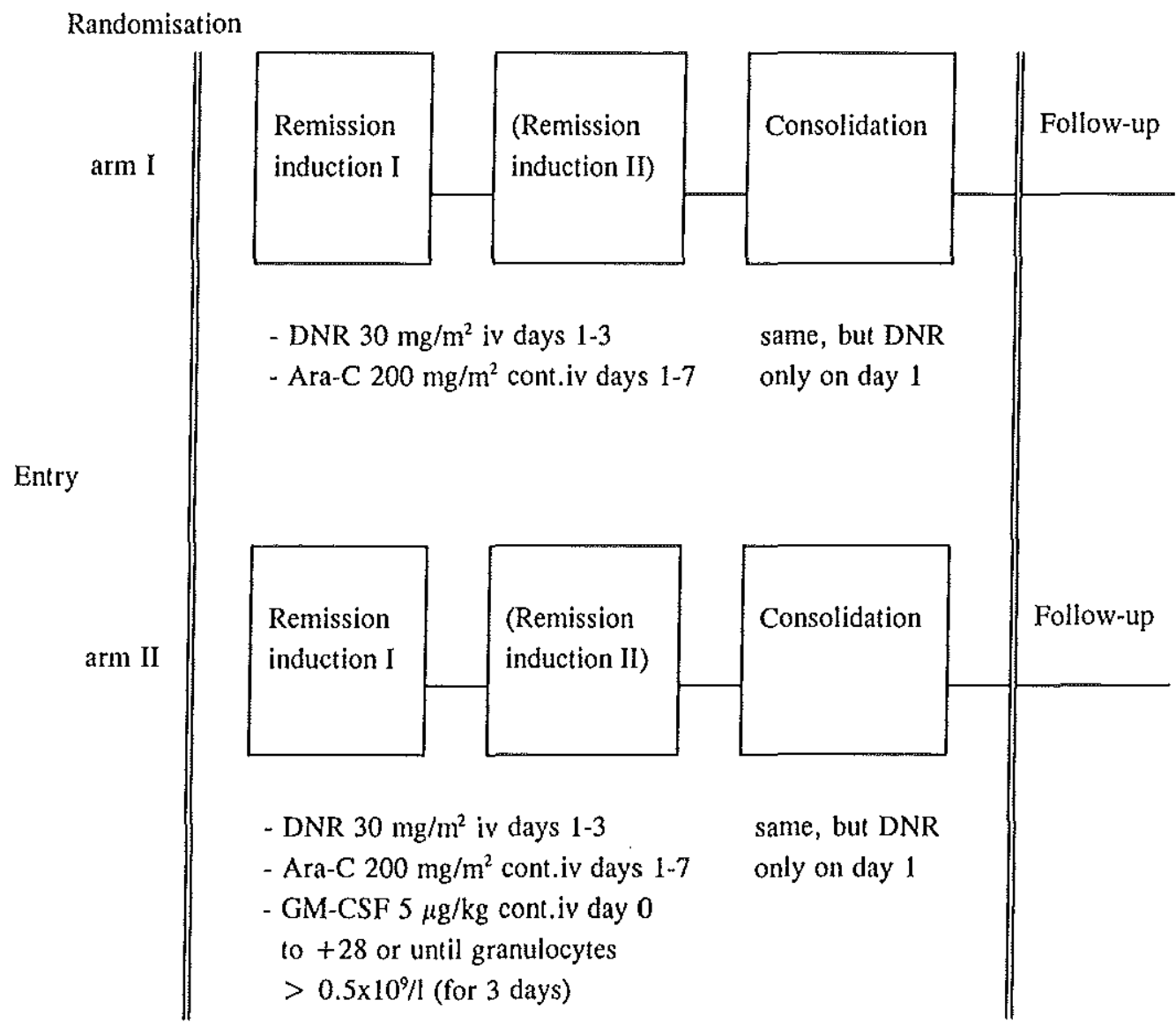

Attempts were made before, during, and following chemotherapy to control any medical problems such as bleeding, infection, and metabolic abnormalities. Patients received platelet transfusions for haemorrhages. During the chemotherapy the patients were placed on a prophylactic regimen of transfusion to keep platelet counts above $10 \times 10^{9} / 1$. Patients with fever received broad-spectrum antibiotics intravenously. Antibiotics were adjusted according to the in vitro sensitivity whenever a pathogen had been isolated. Red blood cell support was given to maintain the haematocrit above approximately $30 \%$. 


\subsubsection{Evaluation of outcome}

The primary end point was the effect of GM-CSF on the percentage of complete remissions (CRs). CR was defined as follows: a normocellullar bone marrow containing less than $5 \%$ blast cells including monocytoid cells, less than $10 \%$ blast cells and promyelocytes, and less than $50 \%$ erythroid cells, no evidence of extramedullary leukaemia, and recovery of peripheral blood values to platelet counts of at least $100 \times 10^{9} / 1$ and neutrophils of at least $1.5 \times 10^{9} / 1$.

Partial remissions (PRs) were defined by bone marrow smears containing between $5.1 \%$ and $25.0 \%$ blasts and less than $5 \%$ circulating blast cells. Failures to induction chemotherapy (NoCR) were classified according to Preisler et al. (1979), i.e., drug resistance, regeneration failure, prolonged hypoplasia, hypoplastic death (i.e., after the chemotherapy cycle), and early death (i.e. during induction treatment).

Survival duration was expressed in months (from diagnosis to death) and disease-free survival was expressed as the time interval (in months) between attainment of $\mathrm{CR}$ and relapse or death whatever came first. Frequencies of excessive toxicities, number of nights spent in hospital, frequencies of admission, frequencies of transfusions, frequencies and nature of haemorrhages and infections, number of days towards haematopoietic recovery, number of episodes of fever and infections were evaluated separately.

\subsubsection{Quality of life analysis}

The quality of life of the patients was measured through several patient questionnaires. First, the Karnofsky index was included. This index emphasises the physical performance and dependency. Second, the patients were asked to complete the Nottingham Health Profile. The Nottingham Health Profile was selected as a generic instrument to measure a broad spectrum of health dimensions and can be used over a wide range of disease categories. The following dimensions of well-being were measured: physical mobility, emotional reactions, energy, social isolation, pain and sleep. Third, the Rotterdam Symptom Checklist, a cancer specific questionnaire sensitive to changes in health of patients having cancer, was applied.

As it is preferable in an economic evaluation to value health states in terms of one summary measure, the EuroQol, a valuation instrument, has been added to the questionnaire. This instrument allows the calculation of "quality adjusted life years" (QALYs), 
where a life year gained is "adjusted" using this value judgement for quality of life. A multi-attribute utility function is available to calculate the values of all health states distinguished in the EuroQol questionnaire. The latter function can be used to arrive at utility values for each health state reported by the patients.

Patients were asked to fill out the questionnaires at the start of the first treatment, during hospitalisation (i.e. 2 days after (theoretically) completing GM-CSF treatment) after hospitalisation (when the patient was at home), and because a survival benefit was expected also during follow-up at 6 months, 1 year and 2 years after the date of randomisation.

\subsubsection{Cost analysis}

The cost analysis was based on a detailed review of patient and hospital records. We examined in detail all resources used for the patients from the Dutch hospitals. Costs considered were: days in hospital, outpatient visits, day-care departments, laboratory services, diagnostics (mainly imaging procedures), radiotherapeutical and surgical procedures, blood products, medication, parenteral nutrition and consultations.

For each of these activities unit prices were determined reflecting the real use of resources. The base year for the study was 1992. The costs of hospital days and outpatient visits were divided into direct and indirect costs. The direct costs concerned manpower (doctors, nurses, etc.) and materials (medical devices, supportive patient care etc.). The indirect costs were related to overheads. The costs of hospitalisation amounted to about US $\$ 350$ per day for regular haematological care, US\$536 for staying in a protected environment and US\$1223 for an intensive care ward. An outpatient visit was calculated at about US\$ 87 and a treatment in a day-care department at about US\$134.

The output of laboratories in the Netherlands is scored according to a point system. Each point can be expressed in a cost price per unit. The cost prices differ across types of laboratories. A routine test (including $\mathrm{Hb}, \mathrm{Ht}$, leucocytes and platelets) costed US\$3.58 and a blood culture costed about US $\$ 20.50$. For most diagnostics the Dutch tariff system has been used as an approximation of unit costs. The cost of the insertion of a central venous catheter was US $\$ 504$ (including the costs of the catheter). The drug prices used were wholesale prices. The costs of a $300 \mu \mathrm{g}$ vial of GM-CSF (intravenous) costed about US\$ 138 and a $400 \mu \mathrm{g}$ vial about US\$184. (Appendix A4.3 for a list of unit prices used in this study). 
The cost analysis distinguishes treatment costs and costs of the follow-up period (maximum of 2 years). A discount rate of $5 \%$ was used.

\subsubsection{Statistical analysis}

The primary end point was the effect of GM-CSF treatment on the percentage of CR. In order to detect an increase of $15 \%(\alpha=0.05)$ in the CR rate from $50 \%$ to $65 \%$, with a power of $85 \%$ the required number of patients was 155 per treatment arm. Therefore, a total of 310 patients were required to be randomised. Approximately 310 patients will also allow to detect a treatment difference from $15 \%$ to $25 \%$ in terms of patients alive at 2 years $(\alpha=0.05$ and $\beta=0.20)$.

For the comparison of the clinical characteristics and the quality of life and cost items the chi-square, the two-tailed Fisher's exact test, the Wilcoxon-Mann-Whitney test and Student's t-test as appropriate were used.

\subsection{Clinical results}

\subsubsection{Response to treatment}

There have been 326 patients registered, 316 were considered as eligible and 10 as ineligible. Reasons of ineligibility were incomplete data $(n=2)$, poor physical condition $(n=3)$ and other malignant disease $(n=5)$. Of the remaining 316 patients, 289 patients have been documented and evaluated at the time of the analysis for this report. Table 9.1 presents the patient characteristics.

CR on the first induction cycle was achieved in 67 patients $(46.2 \%)$ in the control group and in 72 patients $(50.0 \%)$ in the GM-CSF group $(P=0.884)$ (Table 9.2). Of 39 patients with a partial response 35 received a second cycle of induction treatment (19 reached $C R$ ) and 4 patients were "too early" to evaluate. A patient classified as resistant after induction, received a second induction course and reached CR. In total, 26 additional patients reached $\mathrm{CR}$, leading to $163 \mathrm{CRs}$. Reasons for not attaining $\mathrm{CR}$ were related to resistance to chemotherapy, prolonged hypoplasia, death in hypoplasia and early death. In summary, there were no significant differences between the two groups. 
Table 9.1 Patient characteristics

\begin{tabular}{|c|c|c|c|}
\hline & & Control group & GM-CSF group \\
\hline \multirow[t]{2}{*}{ Sex: } & Male & $51.7 \%$ & $55.6 \%$ \\
\hline & Female & $48.3 \%$ & $44.4 \%$ \\
\hline \multirow[t]{3}{*}{ Age (year): } & $60-70$ year & $70.4 \%$ & $68.5 \%$ \\
\hline & $70-80$ year & $28.0 \%$ & $29.0 \%$ \\
\hline & $>80$ year & $1.6 \%$ & $2.4 \%$ \\
\hline \multirow[t]{3}{*}{$W B C^{*}:$} & $<30$ & $70.4 \%$ & $67.5 \%$ \\
\hline & $<100$ & $20.8 \%$ & $22.8 \%$ \\
\hline & $100+$ & $8.8 \%$ & $9.8 \%$ \\
\hline \multirow[t]{8}{*}{$F A B$ cytology: } & Mo & $5.9 \%$ & $5.1 \%$ \\
\hline & M1 & $14.4 \%$ & $20.3 \%$ \\
\hline & $\mathrm{M} 2$ & $43.2 \%$ & $35.6 \%$ \\
\hline & M3 & $1.7 \%$ & $1.7 \%$ \\
\hline & M4 & $12.7 \%$ & $14.4 \%$ \\
\hline & M5 & $17.8 \%$ & $16.1 \%$ \\
\hline & M6 & $4.2 \%$ & $5.1 \%$ \\
\hline & M7 & - & - \\
\hline Total: & & 145 & 144 \\
\hline
\end{tabular}

${ }^{*} \mathrm{WBC}=$ white blood cell count $\left(\times 10^{9} / \mathrm{l}\right)$

Table 9.2 Response to induction and consolidation chemotherapy

\begin{tabular}{|l|r|r|}
\hline & $\begin{array}{r}\text { Control group } \\
(n=145)\end{array}$ & $\begin{array}{r}\text { GM-CSF group } \\
(n=144)\end{array}$ \\
\hline Complete response (\%) & 46.2 & 50.0 \\
After remission induction cycle I & 57.9 & 54.9 \\
After remission induction cycle II & & \\
No complete response (\%) & 28.3 & \\
Resistance to chemotherapy & 0.7 & 27.1 \\
Prolonged hypoplasia & 9.7 & 4.2 \\
Death in hypoplasia & 3.4 & 13.9 \\
Early death & - \\
\hline
\end{tabular}




\subsubsection{Overall survival and disease-free survival}

The duration of survival at 6 months, 1 and 2 years after randomisation were $61 \%, 41 \%$ and $26 \%$, respectively, in the control group and $67 \%, 46 \%$ and $23 \%$, respectively, in the GM-CSF group $(P=0.62)$ (Figure 9.2). Of the 163 complete responders to intensive chemotherapy 12 patients died in CR ( 7 patients in the control group and 5 patients in the GM-CSF group).

Figure 9.2 Overall survival of patients treated with chemotherapy with or without GM-CSF

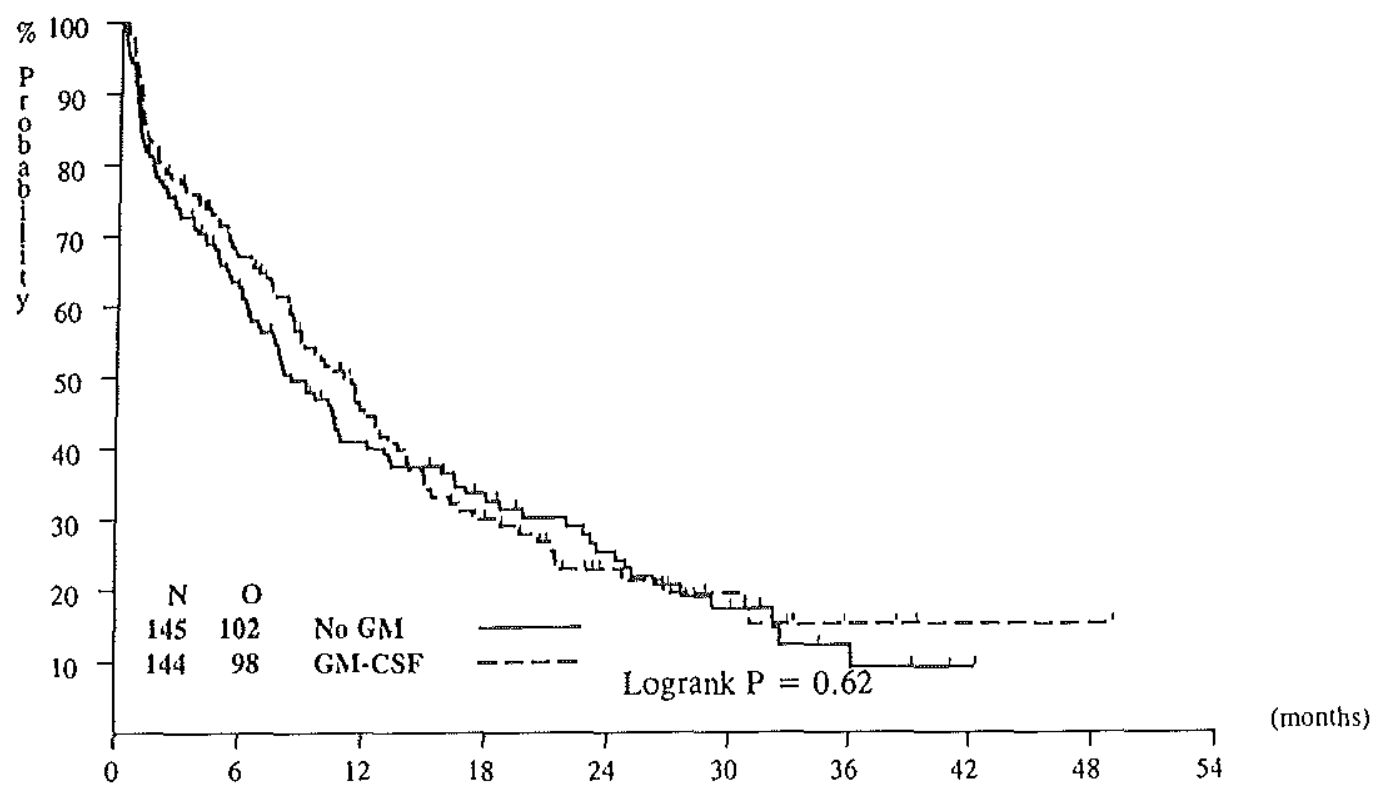

Figure 9.3 shows the disease-free survival. The disease-free survival at 6 months, 1 and 2 years after randomisation were $72 \%, 41 \%$ and $19 \%$ in the control group and $80 \%, 37 \%$ and $11 \%$, respectively, in the GM-CSF group $(P=0.65)$. 
Figure 9.3 Disease-free survival of patients treated with chemotherapy with or without GM-CSF

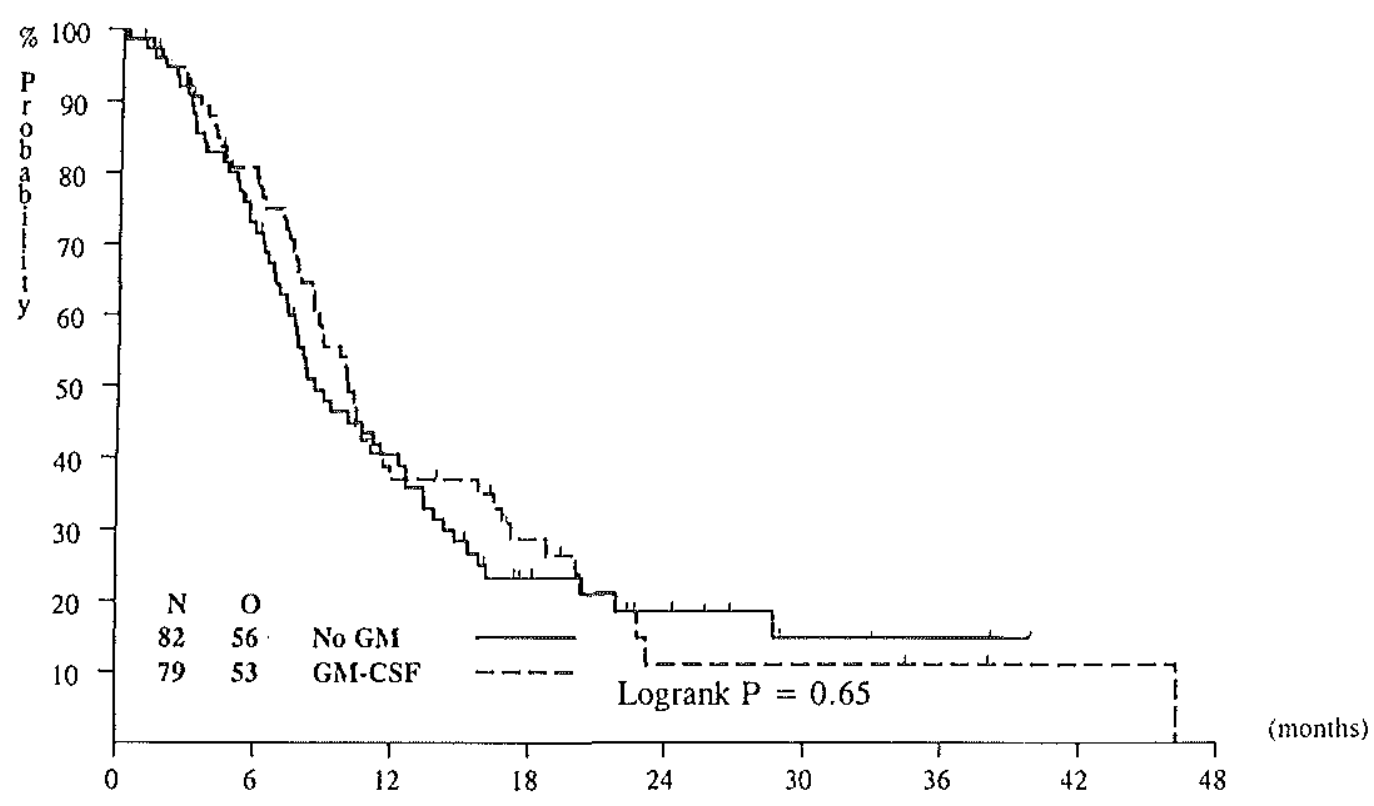

\subsubsection{Hospitalisation, GM-CSF and haematopoietic recovery}

Figure 9.4 shows the duration of hospitalisation during induction cycle I. The median number of days in hospital are 32.0 days in the control group and 32.5 days in the GMCSF group (control group: mean 33.88 and range 3-75; GM-CSF group: mean 34.54 and range: $11-98)(P=0.87)$. The patients in the GM-CSF group received on average 17.78 days GM-CSF treatment (median: 20; range: 0-46). GM-CSF administration started on day 0 in $68.6 \%$ of the patients. In $21.5 \%$ of the patients the GM-CSF administration began between 1.4 days after the start of the chemotherapy, in $7.4 \%$ between 4.6 days and in $2.5 \%$ after more than 6 days. This was in most instances according to the protocol as GM-CSF was required to be postponed in case of white blood cell counts (WBC) of $30 \times 10^{9} / 1$ or more and was indicated to be initiated as soon as WBC had declined to $20 \times 10^{9} / 1$. In $43.5 \%$ of the patients the GM-CSF was interrupted or stopped although the WBC was less than 30 , mainly due to possible toxicity of GM-CSF. 
Figure 9.4 Duration of hospitalisation during induction cycle I.

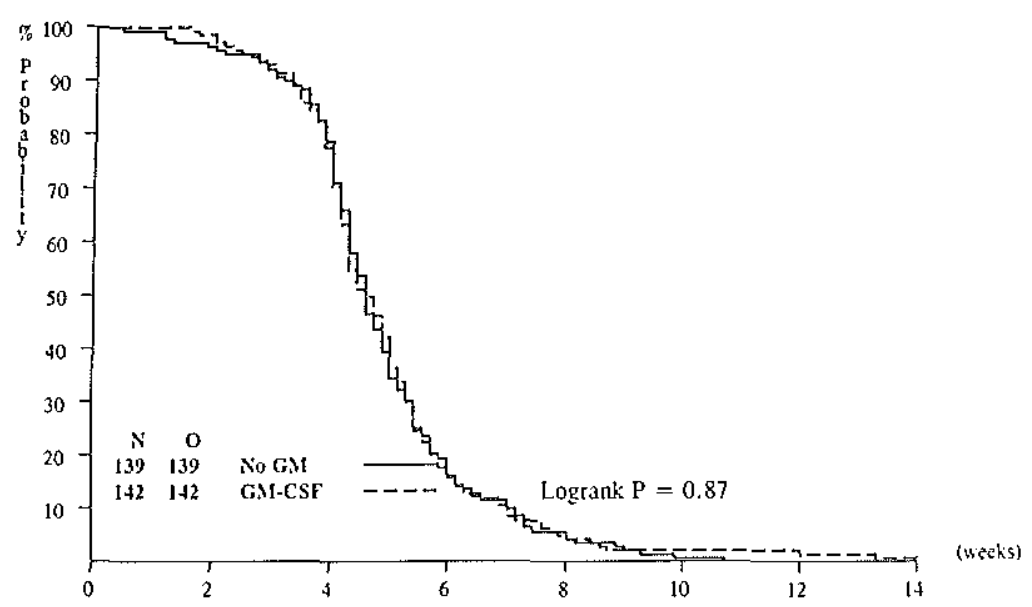

Figures 9.5 and 9.6 present the time to recovery of polymorphonuclear neutrophils (PMN) of 0.5 and $1.0 \times 10^{9} / 1$, from day 1 of treatment in patients who reached CR after induction cycle $\mathrm{I}$. For $\mathrm{PMN}=0.5 \times 10^{9} / 1$ the median time to recovery was estimated at $3.74 \mathrm{wks}$ in the control group and $3.62 \mathrm{wks}$ in the GM-CSF group. The relative risk of GM-CSF versus control is 1.852 and the $95 \% \mathrm{Cl}$ is $(1.23,2.78)(P=0.003)$. The median time of recovery of $\mathrm{PMN}>1.0 \times 10^{9} / 1$ was estimated at $4.36 \mathrm{wks}$ in the control group and $3.75 \mathrm{wks}$ in the GM-CSF group and the relative risk of GM-CSF versus control is 2.145 (CI: $1.42-3.24)(P=0.0003)$. About $15-20 \%$ of the patients in the control group show a long lasting neutropenia and do not met PMN recovery even during 8-12 wks. This is in contrast to the results of the GM-CSF treated patients.

Figure 9.5 Time to PMN $>0.5 \times 10^{\circ} / 1$

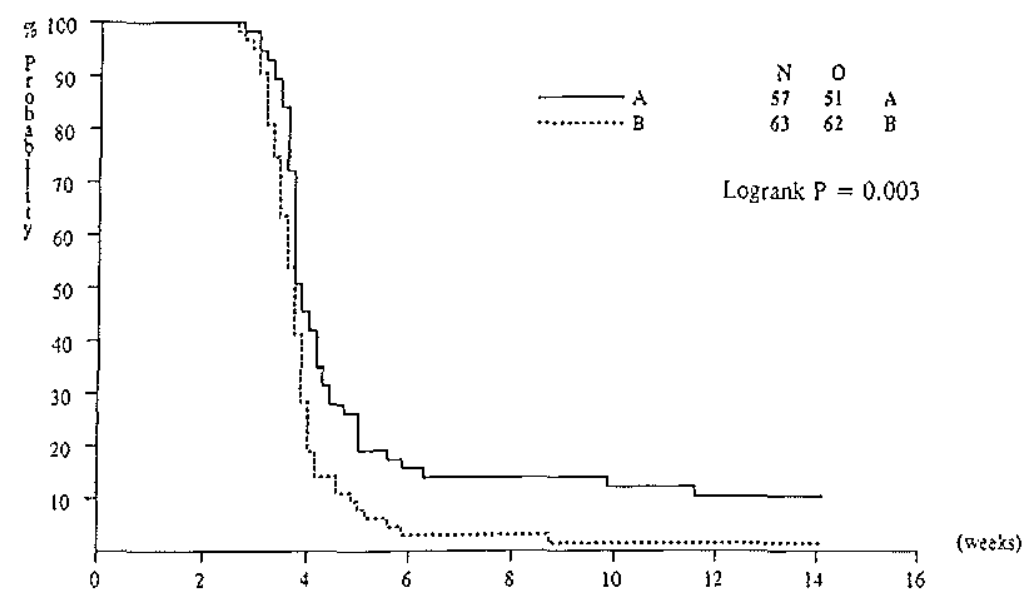


Figure 9.6 Time to PMN $>1.0 \times 10^{9} / 1$

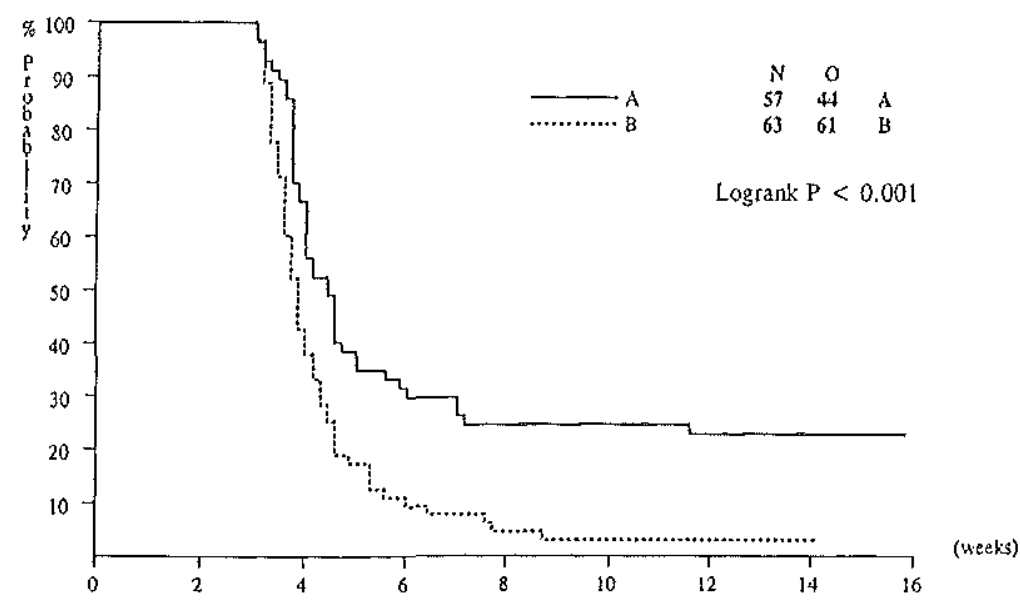

Figures 9.7 and 9.8 show the time of recovery to platelets (PLT) of 20 and $50 \times 10^{9} / 1$ from day 1 of chemotherapy induction cycle I for those who patients who reached CR after induction I. The median time of recovery to PLT $>20 \times 10^{9} / 1$ was 3.17 wks in the control group and $3.21 \mathrm{wks}$ in the GM-CSF. The relative risk of GM-CSF versus control is 1.188 and the $95 \%$ confidence interval $(\mathrm{CI})$ is $(0.80,1.77)(P=0.39)$. The median time to recovery was estimated at $3.61 \mathrm{wks}$ in the control group and 3.68 weeks in the GM-CSF group. The relative risk of GM-CSF versus control is 1.04 (CI: $0.73-1.54)(P=0.84)$.

Figure 9.7 Time to PLT $>20 \times 10^{9} / 1$

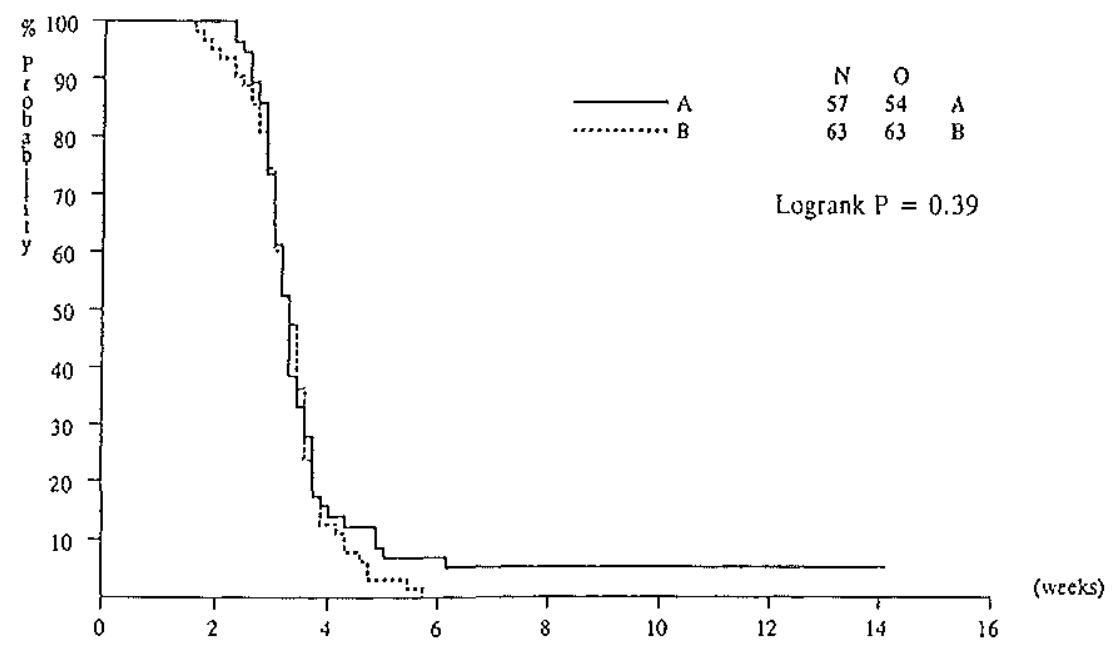


Fígure 9.8 Time to PLT $>50 \times 10^{\circ} / 1$

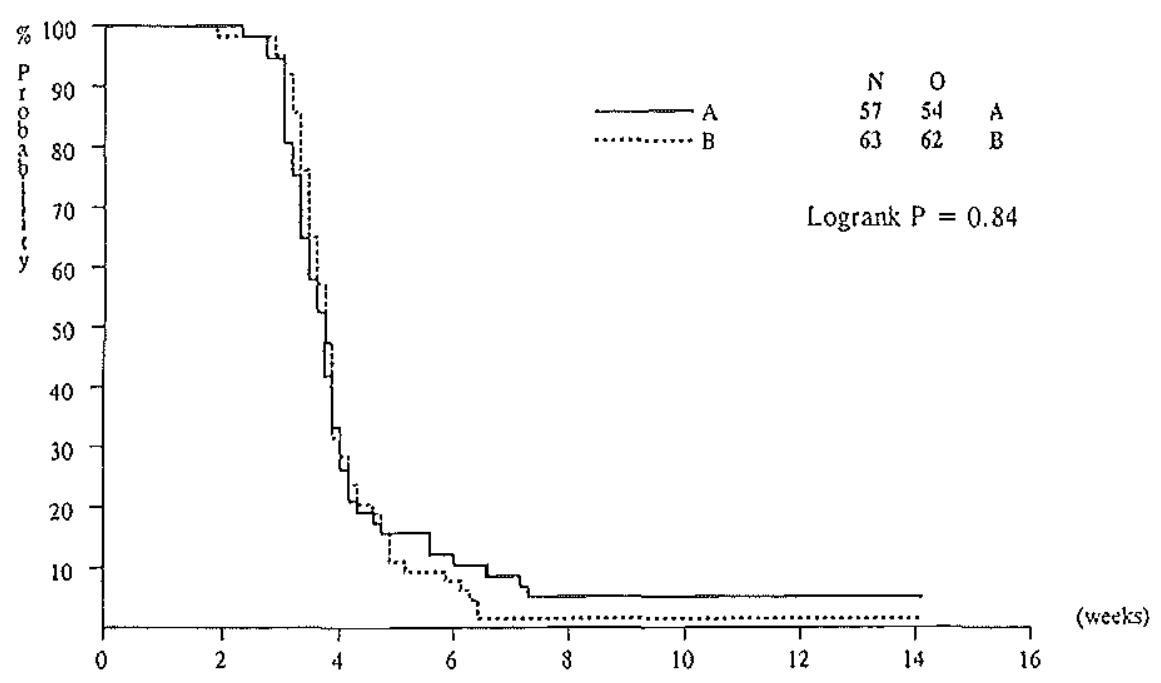

\subsubsection{Fever}

In Figure 9.9 the duration of fever during induction cycle $I$ is presented. The median duration of fever is 6.0 days in the control group (mean: 8.13 and range: $0-41$ ) and 10.0 days in the GM-CSF group (mean: 11.48 and range: $0-40$ ). The relative risk of GM-CSF versus control is 0.603 (CI: $0.465-0.780)(P<0.001)$.

Figure 9.9 Duration of fever during induction cycle I

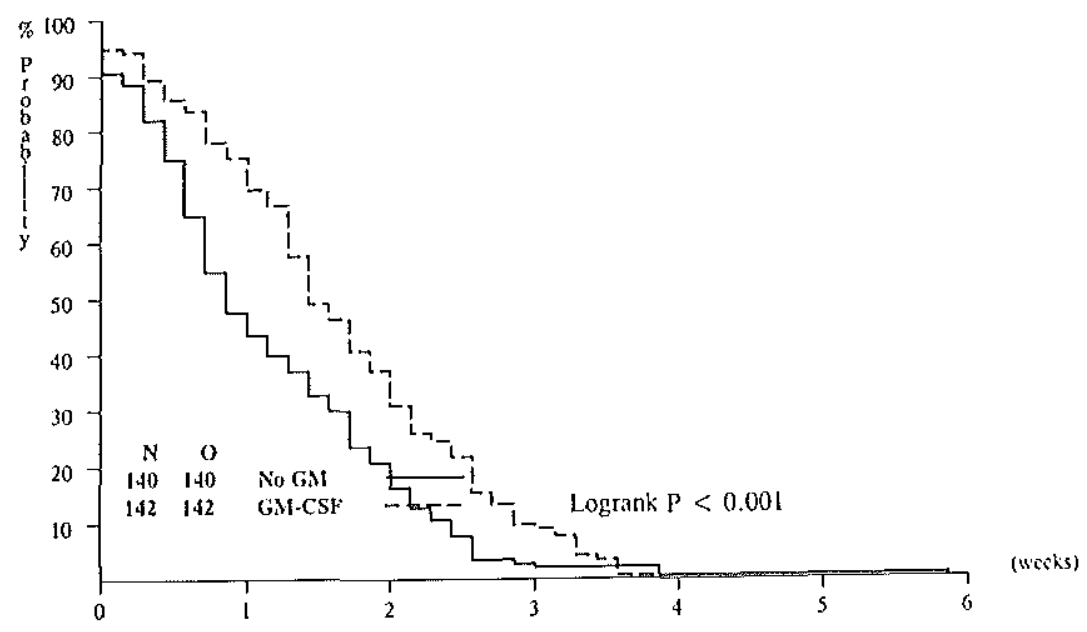




\subsubsection{Supportive care}

The patients in the control group received on average 18.12 days antibiotic treatment (median: 16.0; range: $0-80$ ). In the GM-CSF group antibiotics were administered on average 19.9 days (median: 20.0 ; range: $0-40$ ). The patients in the control group got on average 21.18 platelet transfusions (median: 12.0 ; range: $0-100$ ). The number of platelet transfusions was classified as follows: 19.45 pooled donor and 1.74 single donor platelet transfusions. The patients in the GM-CSF group received on average 18.41 pooled donor and 2.65 single donor platelet transfusions, i.e. on average 21.06 platelet transfusions (median: 12.0; range: $0-99)(P=N S)$. The average number of red cell blood transfusions was not significantly different in the control group as compared with the GM-CSF group, 12.46 (median: 11.0; range 2-40) versus 13.83 (median: 13.0; range: 2-48).

\subsubsection{Toxicity}

Table 9.3 shows the relative frequency of complications during induction cycle I. The frequencies of haemorrhages, liver abnormalities, oral toxicity, nausea, cardiac rhythm abnormalities, neurotoxicity, bone pain, phlebitis and infections were similar in both groups. However, the patients in the GM-CSF group experienced more diarrhoea, renal abnormalities, fever, cutaneous toxicity, hypotension, fluid retention and chills.

Table 9.3 Relative frequency of complications during induction cycle I

\begin{tabular}{|l|r|r|}
\hline & $\begin{array}{r}\text { Control } \\
\text { group }\end{array}$ & $\begin{array}{r}\text { GM-CSF } \\
\text { group }\end{array}$ \\
\hline $\begin{array}{l}\text { Haemorrhage } \\
-\quad \text { no }\end{array}$ & 50.4 & \\
$-\quad$ only petechiae & 23.6 & 49.2 \\
$-\quad$ other bleedings & 26.0 & 27.0 \\
\hline Liver & & 23.8 \\
$-\quad$ no abnormalities & 63.1 & \\
$-\quad$ mild $(1.26-5 \times$ normal $)$ & 26.2 & 54.1 \\
$-\quad$ severe $(\geq 5 \times$ normal $)$ & 10.7 & 35.2 \\
\hline Oral & & 10.7 \\
$-\quad$ no & 76.0 & \\
$-\quad$ erythema & 17.4 & 72.1 \\
$-\quad$ ulcers and other toxicity & 6.6 & 16.4 \\
\hline
\end{tabular}




\begin{tabular}{|c|c|c|}
\hline & $\begin{array}{r}\text { Control } \\
\text { group }\end{array}$ & $\begin{array}{r}\text { GM-CSF } \\
\text { group }\end{array}$ \\
\hline \multicolumn{3}{|l|}{ Nausea } \\
\hline- no & 33.3 & 35.0 \\
\hline - yes & 66.7 & 65.0 \\
\hline \multicolumn{3}{|l|}{ Diarrhoea' } \\
\hline- no & 59.0 & 45.5 \\
\hline - $\quad$ less than 2 days & 22.1 & 25.2 \\
\hline - $\quad$ more severe & 18.9 & 29.3 \\
\hline \multicolumn{3}{|l|}{ Renal $^{1}$} \\
\hline normal & 79.0 & 62.3 \\
\hline$-\quad \operatorname{mild}(1.26-2 \times$ normal $)$ & 19.4 & 34.4 \\
\hline - $\quad$ moderate and severe ( $\geq 2 \times$ normal $)$ & 1.6 & 3.3 \\
\hline \multicolumn{3}{|l|}{ Fever $^{1}$} \\
\hline- no & 15.6 & 8.1 \\
\hline - $\quad$ subfebrile $\left(<38^{\circ} \mathrm{C}\right)$ & 13.1 & 12.1 \\
\hline$-\quad \geq 38^{\circ} \mathrm{C}$ & 71.3 & 79.8 \\
\hline \multicolumn{3}{|l|}{ Cutaneous $^{2}$} \\
\hline no & 59.8 & 41.8 \\
\hline - $\quad$ erythema/desquamation/papules etc. & 40.2 & 58.2 \\
\hline \multicolumn{3}{|l|}{ Cardiac rhythm } \\
\hline - $\quad$ no abnormalities & 76.0 & 76.7 \\
\hline - $\quad$ abnormalities & 24.0 & 23.3 \\
\hline \multicolumn{3}{|l|}{ Neurotoxicity } \\
\hline- no & 88.5 & 86.9 \\
\hline$-\quad$ yes & 11.5 & 13.1 \\
\hline \multicolumn{3}{|l|}{ Bone pain } \\
\hline$-\quad$ no & 93.4 & 90.2 \\
\hline - yes & 6.6 & 9.8 \\
\hline \multicolumn{3}{|l|}{ Hypotension ${ }^{2}$} \\
\hline$-\quad$ no & 88.6 & 69.4 \\
\hline - $\quad$ yes & 11.4 & 30.6 \\
\hline \multicolumn{3}{|l|}{ Fluid retention ${ }^{2}$} \\
\hline - no & 43.1 & 25.8 \\
\hline - yes & 56.9 & 74.2 \\
\hline
\end{tabular}




\begin{tabular}{|l|r|r|}
\hline & $\begin{array}{r}\text { Control } \\
\text { group }\end{array}$ & $\begin{array}{r}\text { GM-CSF } \\
\text { group }\end{array}$ \\
\hline Chills & & \\
$-\quad$ no & 86.1 & 64.8 \\
$-\quad$ yes & 13.9 & 35.2 \\
\hline Phlebitis & & \\
$-\quad$ no & 91.0 & 85.5 \\
$-\quad$ yes & 9.0 & 14.5 \\
\hline Infection & & \\
$-\quad$ no & 23.0 & 23.4 \\
$-\quad$ yes & 77.0 & 76.6 \\
\hline
\end{tabular}

$$
{ }^{1} P<0.05 ;{ }^{2} P<0.01
$$

\subsection{Results quality of life study}

\subsubsection{Short-term quality of life}

Table 9.4 presents the quality of life of the patients at the start of the induction treatment, during hospitalisation and after hospitalisation for induction cycle $I$. At the start of the induction treatment there were no significant differences between the two groups. The scores on the Karnofsky, the EuroQol patient and the EuroQol population scores varied between 65 and 77 . With respect to the Nottingham Health Profile, patients in both groups experienced problems with energy and sleep. The most prominent complaints/ symptoms on the Rotterdam Symptom Checklist were tiredness, lack of energy, worrying, nervousness and lack of appetite.

During hospitalisation the patients in the GM-CSF had more frequent problems as compared to the patients in the control group. The Karnofsky score, the EuroQol patient score and the population score in the control group were 76,67 and 72, respectively, and in the GM-CSF group 63,54 and 63, respectively. Concerning the Nottingham Health Profile mobility and lack of energy represent the main problems in both groups. However, taking into account the results of the Rotterdam Symptom Checklist, the patients in the GM-CSF treatment arm more frequently experienced "lack of energy" than did the patients in the control group $(P<0.05)$. Depressed mood, diarrhoea and rash/eczema were less frequently seen on average in the control group. The greater frequency of diarrhoea and rash/eczema corresponded with the results of the toxicity analysis. All patients had "lack of appetite" and "loss of hair". 
Table 9.4 Mean scores of quality of life in both treatment groups during induction I

\begin{tabular}{|c|c|c|c|c|c|c|}
\hline & \multicolumn{2}{|c|}{$\begin{array}{l}\text { Start induction } \\
\text { treatment }\end{array}$} & \multicolumn{2}{|c|}{$\begin{array}{c}\text { During } \\
\text { hospitalisation }\end{array}$} & \multicolumn{2}{|c|}{$\begin{array}{l}\text { After hospitalisation } \\
\text { (at home) }\end{array}$} \\
\hline & $\begin{array}{r}\text { Control } \\
\text { group } \\
(n=10)\end{array}$ & $\begin{array}{r}\text { GM-CSF } \\
\text { group } \\
(n=11)\end{array}$ & $\begin{array}{r}\text { Control } \\
\text { group } \\
(n=20)\end{array}$ & $\begin{array}{r}\text { GM-CSF } \\
\text { group } \\
(n=10)\end{array}$ & $\begin{array}{r}\text { Control } \\
\text { group } \\
(n=14)\end{array}$ & $\begin{array}{r}\text { GM-CSF } \\
\text { group } \\
(n=8)\end{array}$ \\
\hline Karnofsky Index & 72.7 & 72.0 & 75.5 & 63.0 & 84.3 & 72.5 \\
\hline $\mathrm{NHP}^{2}$ & & & & & & \\
\hline - Mobility & 11.5 & 12.2 & 16.9 & 27.4 & 10.5 & 28.0 \\
\hline - Emotional reactions & 8.7 & 18.2 & 8.4 & 16.7 & 5.6 & 6.1 \\
\hline - Energy & 26,9 & 23.5 & 25.7 & 25.9 & 15.9 & 29.1 \\
\hline - Social isolation & 5.5 & 8.6 & 4.1 & 8.3 & 2.9 & 0.0 \\
\hline - Pain & 5.9 & 6.0 & 4.2 & 14.5 & 3.1 & 4.5 \\
\hline - Sleep & 18.3 & 24.8 & 9.1 & 38.3 & 15.1 & 15.1 \\
\hline EuroQol $^{1}$ & & & & & & \\
\hline - Patient score & 70.6 & 64.8 & 67.1 & 53.5 & 72.7 & 68.0 \\
\hline - Population score & 67.5 & 77.4 & 72.1 & 62.8 & 79.1 & 66.3 \\
\hline Complaints $\mathrm{RSCL}^{4}$ & & & & & & \\
\hline 1. Tiredness & 2.2 & 1.8 & 1.9 & 2.6 & 2.0 & 2.3 \\
\hline 2. Lack of energy & 1.9 & 1.9 & $1.5^{*}$ & 2.5 & 1.4 & 1.4 \\
\hline 3. Worrying & 1.9 & 2.0 & 1.5 & 2.0 & 1.3 & 1.6 \\
\hline 4. Depressed mood & 1.3 & 1.6 & $1.4^{*}$ & 1.6 & 1.2 & 1.3 \\
\hline 5. Nervousness & 1.9 & 2.2 & 1.6 & 1.8 & 1.6 & 1.6 \\
\hline 6. Lack of appetite & 1.9 & 2.0 & 2.0 & 2.7 & 1.5 & 1.3 \\
\hline 7. Nausea & 1.2 & 1.7 & 1.6 & 2.1 & 1.4 & 1.1 \\
\hline 8. Diarrhoea & 1.3 & 1.1 & $1.1^{\circ}$ & 1.9 & 1.0 & 1.0 \\
\hline 9. Loss of hair & 1.1 & 1.1 & 2.7 & 2.8 & 2.4 & 2.4 \\
\hline 10. Shivering & 1.1 & 1.3 & 1.4 & 1.9 & 1.3 & 1.3 \\
\hline 11. Rash, eczema & 1.4 & 1.2 & $1.5^{\circ}$ & 3.2 & 1.4 & 1.5 \\
\hline 12. Painful joints & 1.2 & 1.4 & 1.3 & 1.2 & 1.4 & 1.4 \\
\hline
\end{tabular}

1 Range 0-100: from worst (0) to best health states (100); ${ }^{2}$ Range 100-0: from worst (100) to best health states $(0) ;{ }^{3}$ Utility scores based on the valuations of patients and of a representative panel of the population; ${ }^{4}$ Answer possibilities: $1=$ not at all, $2=$ a little, $3=$ quite a bit and $4=$ very much. The average values are presented; $" P<0.05$. 
At home (after hospitalisation) the patients experienced fewer restrictions with respect to quality of life. The Karnofsky scores were 84 in the control group and 73 in the GM-CSF group. The EuroQol patient and population scores were 73 and 79 in the control group and 68 and 66 in the GM-CSF group. Considering the Nottingham Health Profile patients still had significant problems with energy, sleep and mobility.

\subsubsection{Long-term quality of life}

At 6 months the quality of life scores of the control patients and GM-CSF treated patients did not differ (Table 9.5). The Karnofsky score, the EuroQol patient score and the EuroQol population score at 6 months were 90, 84 and 89 in the control group $(n=14)$ and 90, 81 and 89 in the GM-CSF group $(n=15)$.

Table 9.5 Mean scores of quality of life in both treatment arms in the follow-up period

\begin{tabular}{|l|r|r|r|r|}
\hline & \multicolumn{2}{|c|}{6 months } & \multicolumn{2}{|c|}{1 year } \\
\hline & $\begin{array}{r}\text { Control } \\
\text { group } \\
(n=14)\end{array}$ & $\begin{array}{r}\text { GM-CSF } \\
\text { group } \\
(n=15)\end{array}$ & $\begin{array}{r}\text { Control } \\
\text { group } \\
(n=10)\end{array}$ & $\begin{array}{r}\text { GM-CSF } \\
\text { group } \\
(n=8)\end{array}$ \\
\hline Karnofsky Index & 90.0 & 90.0 & 83.0 & 82.5 \\
Nottingham Health Profile & & & & \\
- Mobility & 3.0 & 1.7 & 8.2 & 5.9 \\
- Enotional reactions & 1.0 & 1.4 & 6.2 & 7.9 \\
- Energy & 15.7 & 9.0 & 23.9 & 15.8 \\
- Social isolation & 0.0 & 0.0 & 6.1 & 2.8 \\
- Pain & 3.2 & 2.6 & 7.8 & 16.1 \\
- Sleep & 3.6 & 2.6 & 3.9 & 20.4 \\
EuroQol: patient score & 84.4 & 80.6 & 75.0 & 74.4 \\
EuroQol: population score & 89.0 & 88.9 & 83.2 & 82.6 \\
\hline
\end{tabular}

At 1 year the Karnofsky scores and the EuroQol scores were almost equivalent. However, the patients in the GM-CSF group scored on average better on the dimension energy and scored worse with regard to the dimensions pain and sleep. As at 2 years the number of patients in CR and patients who are not in CR differed between the two groups, these scores are not presented. 
In Table 9.6 the average quality of life scores of patients in CR and patients in NoCR are presented. At all time intervals the Karnofsky scores of the patients in CR were $\geq 90$ and are similar to the scores in the general (healthy) population. The Karnofsky scores of the patients in $\mathrm{CR}$ at 6 months and 1 year are significantly better than the scores of the patients in NoCR. Although the scores at 2 years correspond with the scores at 6 months and 1 year, there were too little observations to notice a significant difference.

Table 9.6 Average quality of life scores per remission state in the follow-up period

\begin{tabular}{|c|c|c|c|c|c|c|}
\hline & \multicolumn{2}{|c|}{6 months } & \multicolumn{2}{|c|}{1 year } & \multicolumn{2}{|c|}{2 years } \\
\hline & $\begin{array}{r}\mathrm{CR} \\
(n=22)\end{array}$ & $\begin{array}{l}\text { NoCR } \\
(n=7)\end{array}$ & $\begin{array}{r}\text { CR } \\
(n=13)\end{array}$ & $\begin{array}{l}\text { NoCR } \\
(n=5)\end{array}$ & $\begin{array}{r}\mathrm{CR} \\
(n=4)\end{array}$ & $\begin{array}{l}\text { NoCR } \\
(n=4)\end{array}$ \\
\hline Karnofsky Index & $94.1^{*}$ & 77.1 & $90.0^{\circ}$ & 64.0 & 95.0 & 70.0 \\
\hline Nottingham Health Profile & & & & & & \\
\hline - Mobility & 3.0 & 0.3 & 1.2 & 22.8 & 0.3 & 23.5 \\
\hline - Emotional reactions & 0.6 & 3.0 & $2.3^{\circ}$ & 19.0 & $0.0^{*}$ & 27.3 \\
\hline - Energy & $5.5^{\circ}$ & 33.6 & $4.9^{*}$ & 60.4 & 6.0 & 31.0 \\
\hline - Social isolation & 0.0 & 0.0 & 1.5 & 12.8 & 0.0 & 16.0 \\
\hline - Pain & 3.8 & 0.0 & 12.9 & 8.0 & 12.0 & 6.5 \\
\hline - Sleep & 4.1 & 0.0 & $5.6^{\circ}$ & 25.8 & 17.8 & 6.5 \\
\hline EuroQol & & & & & & \\
\hline - Patient score & $87.2^{*}$ & 68.9 & 80.4 & 56.3 & 81.3 & 59.5 \\
\hline - Population score & $91.8^{\circ}$ & 80.1 & $92.0^{*}$ & 59.4 & $97.8^{*}$ & 72.5 \\
\hline Complaints RSCL & & & & & & \\
\hline 1. Tiredness & 1.5 & 2.1 & 1.8 & 2.2 & 2.0 & 2.3 \\
\hline 2. Lack of energy & $1.2^{*}$ & 1.9 & 1.3 & 2.0 & 1.3 & 2.3 \\
\hline 3. Worrying & 1.3 & 1.3 & $1.4^{*}$ & 2,8 & 1.8 & 2.3 \\
\hline 4. Depressed mood & 1.1 & 1.3 & 1.2 & 1.8 & 1.0 & 2.3 \\
\hline 5. Nervousness & 1.3 & 1.1 & 1.7 & 2.2 & 1.3 & 2.3 \\
\hline 6. Lack of appetite & 1.0 & 1.0 & $1.1^{*}$ & 2.2 & 1.0 & 1.8 \\
\hline 7. Nausea & 1.1 & 1.0 & 1.1 & 1.2 & 1.3 & 1.3 \\
\hline 8. Diarrhoea & 1.1 & 1.0 & 1.1 & 1.2 & 1.0 & 1.3 \\
\hline 9. Loss of hair & 1.1 & 1.1 & 1.2 & 1.0 & 1.0 & 1.0 \\
\hline 10. Shivering & 1,1 & 1.0 & 1.2 & 1.4 & 1.0 & 1.5 \\
\hline 11. Rash, eczema & $1.1^{*}$ & 1.6 & 1.2 & 1.6 & 1.3 & 1.0 \\
\hline 12. Painful joints & 1.5 & 1.7 & 1.9 & 2.0 & 2.0 & 1.5 \\
\hline
\end{tabular}

${ }^{*} P<0.05$. 
At 6 months the patients in NoCR experienced more problems with energy (both on the Nottingham Health Profile and Rotterdam Symptom Checklist). Furthermore, the EuroQol population scores were better in the patients who were in CR. At 1 year not only the Karnofsky score was higher in the CR group, but also the dimensions emotional reactions, energy and sleep of the Nottingham Health Profile, the EuroQol population scores and the complaints/symptoms lack of appetite and worrying of the Rotterdam Symptom Checklist were significantly better in the $\mathrm{CR}$ group. The complaint/symptom "lack of energy" did not differ significantly between the two groups $(P=0.054)$.

\subsection{3 (Quality adjusted) life years}

For the calculation of the life years and QALYs we used the survival and disease-free survival data. Concerning the QALY calculation, we distinguished patients who were in complete remission and patients who were not in complete remission. The utility scores are derived from the EuroQol population scores at 6 months, 1 and 2 years (see Table 9.6). The lifelong consequences of the treatment modalities have not been calculated, as this study only concerned elderly patients and there were no survival benefits, nor in favour of either the GM-CSF group or the control group.

Table 9.7 presents the life years and QALYs in both treatment arms. Considering a follow-up period of 2 years, the cumulative, discounted survival amounted to 0.979 life years in the control group and to 1.007 in the GM-CSF group. The quality adjusted life years (discounted) were 0.800 in the control group and 0.816 in the GM-CSF group. Thus there are no differences between the two groups.

Table 9.7 (Quality adjusted) life years in both treatment groups

\begin{tabular}{|l|r|r|}
\hline Follow-up: 2 years & $\begin{array}{r}\text { Control } \\
\text { group } \\
(n=145)\end{array}$ & $\begin{array}{r}\text { GM-CSF } \\
\text { group } \\
(n=144)\end{array}$ \\
\hline Life years & 0.995 & 1.023 \\
Life years discounted & 0.979 & 1.007 \\
& & \\
QALYs & 0.813 & 0.828 \\
QALYs discounted & 0.800 & 0.816 \\
\hline
\end{tabular}




\subsection{Results cost analysis}

\subsubsection{Costs of induction and consolidation treatment}

We examined in detail all resources used for 109 patients; 54 patients in the control group and 55 patients in the GM-CSF group. Two patients in the GM-CSF group and four patients in the control group were analysed separately. One patient of the GM-CSF group went off protocol and a second patient received induction treatment I and II during the same hospitalisation period. In the control group, three patients received several courses during their first stay in hospital and one patient was submitted to get a cholecystectomy. Thus, the results reported below are based on 103 patients.

\section{Costs of induction cycle I}

During the remission induction I treatment, the patients in the control group had a mean hospital stay of 36.8 days (median: 36 ; range $3-63$ ). The hospital care was divided as follows: $84 \%$ regular haematological care and $16 \%$ protected environment care. The patients in the GM-CSF group spent 37.9 days on average in the hospital (median: 35; range: 3-69). The hospital stay was divided in $84 \%$ regular haematological care, $14 \%$ care in a protected environment and $2 \%$ intensive care. Table 9.8 presents the average costs of the first induction treatment per category of costs.

Table 9.8 Average costs of induction cycle I

\begin{tabular}{|l|r|r|}
\hline & Control & GM-CSF \\
Treatment & group & group \\
\hline Remission induction I & & \\
Hospitalisation & 14270 & 14950 \\
Consultations & 84 & 101 \\
Laboratory services & 2592 & 2854 \\
Medical procedures & 1437 & 1318 \\
Medication (excl. GM-CSF) & 4412 & 5327 \\
GM-CSF & 0 & 3314 \\
Nutrition & 328 & 434 \\
Transfusions & 2113 & 2301 \\
Total remission induction I & 25236 & 30599 \\
\hline
\end{tabular}


The costs of hospitalisation in the control group were US\$ 14270 and in the GM-CSF group US $\$ 14950(P=\mathrm{NS})$. The costs of laboratory services, diagnostics, nutrition and transfusions did not differ between the control group and the GM-CSF group. The costs of medication amounted to US\$ 4412 in the control group and to US\$5327 in the GMCSF group. The administration of GM-CSF costed about US $\$ 3314$.

The total treatment costs in the control group amounted to US\$25236 (range: US\$2841 US $\$ 50446$ ) and in the GM-CSF group to US\$ 30599 (range: US\$ 3068 - US\$ 56657) $(\mathrm{P}<0.01)$.

\section{Costs of induction cycle II and consolidation treatment}

Patients who received a second remission induction treatment spent less time in the hospital. Control patients spent on average 33.2 days (median: 30.5 ; range: $21-48$ ) in the hospital and GM-CSF patients 33.3 days (median: 32; range: 23-53). The hospital stay of the patients in the control group was classified as follows: $72 \%$ regular haematological care, $22 \%$ care in a protected environment and $6 \%$ intensive care. In the GM-CSF group these percentages were $68 \%, 32 \%$ and $0 \%$, respectively. Although the mean hospital stay during the second induction treatment was similar in both groups, namely 33 days, the costs of hospitalisation were higher in the control group. This difference was caused by the fact that the patients in the control group spent more time in the intensive care ward than did the patients in the GM-CSF group. The hospital costs amounted to US\$15068 in the control group and to US\$13874 in the GM-CSF group (see Table 9.9). The total costs of the second induction treatment were estimated at US $\$ 22965$ in the control group and at US\$25054 in the GM-CSF group.

For the consolidation treatment the mean hospital stay was 28.6 days (median: 29.5; range: $22-36$ ) in the control group and 31.6 days (median: 29; range: 20-64) in the GMCSF group. The hospital stay of the patients in the control group were divided in $86 \%$ regular haematological care and $14 \%$ care in a protected environment. In the GM-CSF group these percentages were $80 \%$ and $20 \%$, respectively. The total costs of the consolidation treatment amounted to US\$15384 in the control group and to US\$20891 in the GM-CSF group (see Table 9.9). 
Table 9.9 Average costs of induction cycle II and consolidation treatment

\begin{tabular}{|l|r|r|}
\hline & Control & GM-CSF \\
Treatment & group & group \\
\hline Remission induction II & & \\
Hospitalisation & 15068 & 13874 \\
Consultations & 62 & 43 \\
Laboratory services & 1904 & 2609 \\
Medical procedures & 838 & 1123 \\
Medication (excl. GM-CSF) & 3447 & 4100 \\
GM-CSF & 0 & 1441 \\
Nutrition & 327 & 408 \\
Transfusions & 1319 & 1456 \\
Total remission induction $I I$ & 22965 & 25054 \\
& & \\
Consolidation & & \\
Hospitalisation & 10916 & 12457 \\
Consultations & 19 & 75 \\
Laboratory services & 1243 & 1657 \\
Medical procedures & 381 & 653 \\
Medication (excl. GM-CSF) & 1942 & 2467 \\
GM-CSF & 0 & 1826 \\
Nutrition & 38 & 395 \\
Transfusions & 845 & 1361 \\
Total consolidation & 15384 & 20891 \\
\hline
\end{tabular}

Remission Induction II: GM-CSF group: $n=10$; control group: $n=12$; Consolidation; GM-CSF group; $n=27$; control group: $n=22$.

The average treatment costs largely depended on the percentages of patients who received a second remission treatment and the percentages of patients who received a consolidation treatment. For the calculation we used the percentages of the whole study population $(n=289)$. In the control group $12.2 \%$ of patients received a second induction treatment and in the GM-CSF group $7.7 \%$ of the patients received a second induction treatment (see Table 9.10). The percentages of patients who received a consolidation treatment were $41.7 \%$ and $39.5 \%$, respectively. The average treatment costs were thus estimated at US\$ 34466 in the control group and at US\$ 40782 in the GM-CSF treated patients. 
Table 9.10 Average treatment costs

\begin{tabular}{|l|r|r|r|r|}
\hline & \multicolumn{2}{|c|}{ Control group } & \multicolumn{2}{c|}{ GM-CSF group } \\
\hline & $\begin{array}{r}\text { Percentage } \\
\text { treatment }\end{array}$ & Costs & $\begin{array}{r}\text { Percentage } \\
\text { treatment }\end{array}$ & Costs \\
\hline Remission induction I treatment & $100.0 \%$ & 25236 & $100.0 \%$ & 30598 \\
Remission induction II treatment & $12.2 \%$ & 2813 & $7.7 \%$ & 1932 \\
Consolidation treatment & $41.7 \%$ & 6417 & $39.5 \%$ & 8252 \\
Average treatment costs & & 34466 & & 40782 \\
\hline
\end{tabular}

Note: The costs of the treatment of the patients who were analysed separately, were as follows: The treatment costs of the GM-CSF patient who went off protocol were US $\$ 108831$. The treatment of the patient who received induction treatment $I$ and II during the same hospitalisation period costed US\$ 44971. In the control group, two patients received 2 induction cycles. Their treatment costs were US\$ 52413 and US\$ 94677, respectively. One patient received 2 induction cycles and a consolidation cycle. The treatment costs of this patient amounted to US\$ 64096 . The fourth patient was submitted to get a cholecystectomy immediately after induction cycle I. The treatment costs, including the costs of the cholecystectomy, were US $\$ 72675$. No treatment induced differences in the cost profiles of these patients were apparent.

\subsubsection{Costs of follow-up and total costs of AML treatment}

For the follow-up evaluation, i.e., after completion of the chemotherapy, we considered the follow-up period as half year intervals and distinguished patients who had attained a $C R$ and patients who had failed to enter CR (see Table 9.11). Since discounting requires yearly intervals from start of treatment, the intervals were calculated from the date of randomisation.

For patients in remission in the control group and in the GM-CSF group, the follow-up costs decreased with time. The follow-up costs of the patients not in CR depended on whether or not they received additional chemotherapy and/or transfusions. For the latter group of patients, the costs during the follow-up period varied between US\$ 8594 and US\$ 15327. 
Table 9.11 Costs during 6 months in the follow-up period in both groups

\begin{tabular}{|l|r|r|}
\hline & $\begin{array}{r}\text { Control } \\
\text { group }\end{array}$ & $\begin{array}{r}\text { GM-CSF } \\
\text { group }\end{array}$ \\
\hline Complete remission & & \\
-6 months & $1839(n=27)$ & $1512(n=22)$ \\
-1 year & $1271(n=15)$ & $1392(n=12)$ \\
-1.5 years & $926(n=11)$ & $758(n=10)$ \\
-2 years & $814(n=6)$ & $631(n=5)$ \\
& & \\
No complete remission & & \\
-6 months & $10248(n=16)$ & $8594(n=17)$ \\
-1 year & $13767(n=15)$ & $13659(n=15)$ \\
-1.5 years & $15327(n=9)$ & $9241(n=10)$ \\
-2 years & $9197(n=9)$ & $12942(n=5)$ \\
\hline
\end{tabular}

Table 9.12 presents the average costs of treatment and follow-up. The follow-up costs in the first year amounted to US\$10831 in the control group and to US\$11266 in the GMCSF group. In the second year the follow-up costs were less than in the first year, i.e., US\$ 6571 in the control group and US\$ 6039 in the GM-CSF group. There were no apparent treatment related differences between the cost profiles of control patients and patients on GM-CSF therapy.

Table 9.12 Costs of treatment and follow-up in both groups

\begin{tabular}{|l|r|r|}
\hline & $\begin{array}{r}\text { Control } \\
\text { group }\end{array}$ & $\begin{array}{r}\text { GM-CSF } \\
\text { group }\end{array}$ \\
\hline Treatment costs & 34465 & 40782 \\
Follow-up costs first year & 10831 & 11266 \\
Follow-up costs second year & 6571 & 6039 \\
Total costs & 51867 & 58087 \\
Total costs discounted & 51554 & 57799 \\
\hline
\end{tabular}

The total discounted costs of the control group, consisting of the initial treatment costs and the costs of the follow-up period, amounted to US\$51554 and in the GM-CSF group to US\$57799. 


\subsection{Discussion}

This randomised study considered the role of GM-CSF as an adjunct to chemotherapy in elderly patients with AML. The assumption was that GM-CSF might improve the response of AML to cytotoxic therapy by combining chemotherapy with GM-CSF stimulation. It also was assumed that GM-CSF might possibly mitigate the duration of leucopenia following chemotherapy and thereby reduce the frequency of bacterial and fungal infections and decrease morbidity and/or mortality. The results of this study show that treatment with daunomycin-cytosine arabinoside combined with GM-CSF does not result in better response rates or better survival of patients with AML of 60 years and older. The percentage of patients who had a $\mathrm{CR}$ after induction treatment was $56.8 \%$ in the control group and $56.5 \%$ in the GM-CSF group. Thus apparently the co-administration of GM-CSF with chemotherapy does not increase chemo-sensitivity as the consequence of AML cell "priming". Nor does the use of GM-CSF post chemotherapy reduce the complications during the hypoplastic phase and decrease morbidity or mortality.

There are several possible reasons that might be considered to explain a lack of clinical efficacy of GM-CSF in the context of chemotherapy in patients with AML at higher age. Many patients, due to possible toxicity related to GM-CSF treatment, were withdrawn from GM-CSF treatment, so that in reality a considerable proportion of the patients allocated to the GM-CSF arm of the study did not receive the intended dose or schedule of GM-CSF. These withdrawals from protocol treatment may have minimised any potential difference between the two arms.

A second possible explanation is that while GM-CSF in vitro sensitises human AML to the cytotoxic effects of chemotherapy, in vivo pharmacologic doses greater than tolerable would be needed. If dosaging of GM-CSF is sub-optimal thereby compromising any potential benefit, one would conclude from the results of the study that higher dosages of GM-CSF cannot realistically be applied to this category of patients. This means that other strategies would need to be explored to enhance the efficacy of chemotherapy with the aim of increasing the response rate ("percentages remission") and response duration ("quality of remission"). Approximately $40 \%$ of patients still fails on first-line reinduction chemotherapy and GM-CSF does not offer a solution to this problem.

Thirdly, there was an advantage for GM-CSF treated patients with regard to more rapid neutrophil recovery following chemotherapy, but this difference did not translate into fewer infections, shorter hospitalisation, a lesser morbidity or mortality. Apparently the quantitative benefit with regard to neutrophil recovery is too small to be clinically 
meaningful. There were significant differences in the time of recovery of neutrophils. The time of neutrophil recovery to $>0.5 \times 10^{9}$ and to $>1.0 \times 10^{9}$ were significantly shorter in the GM-CSF group than in the control group. Time of platelet recovery to $>20 \times 10^{9} / 1$ and $>50 \times 10^{9} / 1$ did not differ significantly. The administration of GM-CSF resulted in moderate to severe toxicities attributed to GM-CSF. Adverse events reported were diarrhoea, renal abnormalities, fever, cutaneous abnormalities, hypotension, fluid retention and chills. Patients on GM-CSF experienced more days of fever than did control patients.

The quality of life analysis was in accordance with the toxicity analysis and revealed lower scores for patients assigned to GM-CSF therapy. Problems during induction treatment I with regard to depressed mood, diarrhoea and rash/eczema were most frequent. As regards the long-term quality of life there were no differences between the GM-CSF and control groups. There were notable differences between patients who had entered $\mathrm{CR}$ and patients who failed to reach $\mathrm{CR}$. This was true regardless whether or not they were on GM-CSF treatment. Patients who were not in remission were often treated with antibiotics and/or received transfusions. Their quality of life scores were considerably lower than the scores of the patients who were in CR. Patients in remission hardly experienced health problems. Their quality of life scores on the Karnofsky Index, Nottingham Health Profile and the EuroQol were comparable with the scores of "healthy" elderly people.

Considering a follow-up period of 2 years, the cumulative, discounted survival amounted to 0.979 life years in the control group and to 1.007 in the GM-CSF group. The quality adjusted survival was 0.800 in the control group and 0.816 in the GM-CSF group.

The quality of life measurement in elderly patients with AML is associated with significant problems that are related to the disease as well as the high age of the target population. For reasons of comparison, we decided to use the same questionnaires as in a study with patients with aggressive non-Hodgkin's lymphoma who were randomised for ABMT or conventional chemotherapy and in a study with patients with chemotherapy- induced fever and neutropenia. However, contrary to these studies, for some of the AML patients the completion of the questionnaires appeared to be too much a burden. Reasons were the duration of the questionnaire and motivation (assistance of a nurse was sometimes necessary). These problems appear specific for this aged category of patients and resulted in refusals to further cooperate in the quality of life study. For future research we would like to pay more attention to alleviate the burden of completion of the questionnaires, especially for the elderly. A solution may be to use different questionnaires for the shortand long-term quality of life. For the short-term quality of life measurement, it is possible 
to use a cancer-specific questionnaire (e.g. the Rotterdam Symptom Checklist or the EORTC QLQ-C30) in combination with the EuroQol. Before the start of the treatment and for the long-term quality of life, a generic instrument (e.g. the Nottingham Health Profile or the Short-Form 36) in combination with the EuroQol may be used.

Another problem was the time of administration of the questionnaires. The questionnaires were given 2 days after (theoretically) stopping GM-CSF. However, GM-CSF was interrupted or stopped too early in a high percentage of patients. These patients did not fill out the questionnaire. This may have biased the results, as the quality of life of these patients has not been taken into account. Since, the main reason for interrupting/stopping GM-CSF was toxicity, the quality of life of the GM-CSF treated patients may have been overestimated. Nevertheless, since the results were already slightly worse for GM-CSF treated subjects, this most likely would not have changed the overall conclusions.

The treatment of elderly patients with AML is a costly treatment, as specialised care units and extensive supportive care are required. The average costs of the primary treatment were greater in the GM-CSF group than in the control group, namely US\$40782 and US $\$ 34466$, respectively. The cost difference was mainly caused by the costs of the GMCSF administration. The costs during the follow-up period did not differ between the two groups. They were about US\$ 11000 during the first year of follow-up and about US\$ 6000 during the second year.

Based on the results of the study reported here, we conclude that daunomcyin-cytosine arabinoside combined with GM-CSF treatment is not a cost-effective treatment strategy as compared with daunomcyin-cytosine arabinoside alone.

\subsection{State of the art}

The median age of all patients with AML is approximately 60 years, indicative of the fact that most patients with this disease belong to the aged population (Liu Yin, 1993). The general opinion today is that intensive chemotherapy is the treatment of choice for fit elderly patients suffering from AML. A palliative wait-and-see strategy has been shown to be a poor option resulting in frequent hospitalisation and unfavourable survival (Löwenberg et al., 1989). Following intensive chemotherapy, approximately half of the elderly patients with AML will enter a complete remission (a state without clinical and haematologicat evidence of leukaemia) and approximately $1 / 7$ of them will enjoy longterm disease-free survival. However, the best medical management of elderly patients 
with AML is still subject of investigation. The development of a treatment specific for and tailored to the elderly patient with AML appears of utmost importance.

Infections and resistance of AML patients to chemotherapy have been the major causes of death. Therefore, these patients require intensive supportive care during hospitalisation including antibiotics as well as red blood cell and platelet transfusions. There has been hope that the haematopoietic growth factors, especially GM-CSF and G-CSF (and very recently thrombopoietin) might result in improved outcome of medical management either by enhancing the cytotoxicity of the antileukaemic chemotherapy or by reducing the length of post chemotherapy leucopenia or by enhancing the cytotoxicity of the antileukaemic chemotherapy.

Use of myeloid growth factors to accelerate recovery from chemotherapy-induced lelucopenia:

Is there a role for the haematopoietic growth factors to be used following chemotherapy with the objective to hasten myeloid recovery, reduce infections, decrease hospital stay and reduce mortality? The results of our study would indicate that the use of GM-CSF does not result in improved treatment outcome, even though some favourable effect on white blood cell regeneration was noted. The details of our study are described in the text of this report. No benefits of employing GM-CSF were apparent with regard to complete response rate, infection incidence, hospital stay or survival. Recently two other studies in elderly patients with AML, one employing G-CSF and a second study with GM-CSF, confirm the lack of a significant survival advantage for elderly patients treated with GCSF or GM-CSF (Dombret et al., 1995; Stone et al., 1995). Thus, the results of three independent phase III studies do not lend support to the general routine and prophylactic application of these biosynthetic peptides as an integral part of the chemotherapy plan in these individuals. Obviously, this does not exclude the potential utility of these biopharmaceutical drugs in individual cases, e.g. in the treatment of severe infections not responsive to antibiotics.

Use of myeloid growth factors in association with chemotherapy to enhance the cytotoxic effect of chemotherapy:

The myeloid haematopoietic growth factors not only stimulate normal haematopoietic stem cells, but also leukaemic progenitors. It has been shown in vitro that AML cell stimulation with the haematopoietic growth factors GM-CSF or G-CSF would enhance cell killing by chemotherapeutic agents. Based on this, it has been assumed that the administration of GM-CSF or G-CSF concomitantly with chemotherapeutic agents in vivo might augment the antileukaemic efficiency of chemotherapy in patients with AML. As yet, 
there is no evidence to indicate that this effect may be exploited in the clinical setting. Essentially, the results of our study showed identical response rates to chemotherapy whether or not GM-CSF had been added to the chemotherapy. In another parallel study, this time in adult patients with primary AML of less than 60 years, also a lack of clinical benefit was seen in patients treated with GM-CSF. In the latter study (HOVON-4A, preliminary analysis) patients received GM-CSF combined with chemotherapy only (arm 1), or following chemotherapy only (arm 2), or both during and following chemotherapy (arm 3) or finally, they received no GM-CSF at all (arm 4). Thus, all four GM-CSF schedule possibilities were explored. However, again no significant difference was apparent among any of the GM-CSF treated cohorts as far as response and survival outcome is concerned. Thus, for the time being there is no established place for GM-CSF in the standard treatment of patients with AML. Whether this similarly applies to G-CSF is not yet clear. Several studies dealing with priming of AML with other growth factors to enhance antileukaemic therapy are still in progress and the results of these studies properly conducted, need to be awaited.

\section{Thrombopoietin:}

In 1995 a new growth factor, thrombopoietin, will enter clinical trials. Patients with AML submitted to intensive chemotherapy always enter a prolonged period of severe thrombocytopenia, that may last for 5-8 weeks, This severe thrombocytopenia may be associated with bleedings and requires intensive and expensive platelet transfusion support in all individuals. Some patients will become alloimmunised and as a consequence, require HLA selected platelet concentrates for transfusion, a product even more expensive. If such compatible donors cannot be found, in the absence of platelet support, the patient is at serious risk of lethal bleeding. While the studies G-CSF and GM-CSF have not shown positive effects on treatment outcome of patients with AML, it remains to be seen how thrombopoietin might potentially modify prognosis of adult and elderly patients with AML in studies to be conducted in the coming years. 


\section{GM-CSF in chemotherapy-related febrile neutropenia}

\subsection{Summary}

Purpose: To determine whether granulocyte-macrophage colony-stimulating factor (GMCSF) used in addition to standard inpatient antibiotic therapy shortens period of hospitalisation from chemotherapy-induced neutropenic fever.

Patients and methods: 134 patients with a haematological $(n=47)$ or solid tumour $(n=87)$ who had severe neutropenia $\left(<0.5 \times 10^{9} / 1\right)$ and fever $\left(>38.5^{\circ} \mathrm{C}\right.$ once or $>38^{\circ} \mathrm{C}$ twice over a $12 \mathrm{hr}$ observation period) were randomly assigned to receive GM-CSF ( $5 \mu \mathrm{g} / \mathrm{kg} /$ day; $n=65)$ or placebo $(n=69)$ in conjunction with broad-spectrum antibiotics for a minimum of 4 days and a maximum of 14 days. GM-CSF/placebo and antibiotics were stopped if neutrophil count was more than $1.0 \times 10^{9} / 1$ and temperature less than $37.5^{\circ} \mathrm{C}$ during two consecutive days or at a leucocyte count of $10 \times 10^{9} / 1$ or more, both followed by $24 \mathrm{hr}$ observation period (hospitalisation period). Clinical and haematological response as well as quality of life and cost-effectiveness were evaluated. In addition serum cytokine levels were measured at day 0 and 1 of therapy.

Results: Compared with placebo, GM-CSF enhanced the neutrophil recovery. Median neutrophil counts at day 4 were $2.9 \times 10^{9} / 1$ (range $0-25$ ) in the GM-CSF arm and $1.3 \times$ $10^{9} / 1$ (range $\left.0-9\right)$ in the placebo group $(P<0.01)$. No significant difference was observed with regard to median days less than $1.0 \times 10^{9} / 1$ neutrophils $(4 v s)$ or days of fever ( 3 vs 3 ). The median number of days patients were hospitalised while on study was comparable in the GM-CSF group and placebo group, 6 (range 3-14) vs 7 (range 4-14) according to the intention to treat analysis $(P=0.27)$. Multivariate $C o x$ regression analysis after adjustment to all prognostic factors demonstrated a $29 \%$ reduction of the risk of hospitalisation for GM-CSF treatment $(P=0.12)$. Quality of life scores in 90 patients demonstrated significant differences in favour of the placebo group. Hospital costs were 
significantly higher for the GM-CSF treated patients if GM-CSF was included in the price (median costs GM-CSF arm: US $\$ 4140 v s$ placebo arm: US $\$ 3590(P<0.05)$ ). Finally, cytokine measurements of G-CSF, Interleukin-6, and Tumour Necrosis Factor- $\alpha$ demonstrated no significant change due to the application of GM-CSF.

Conclusion: These results indicate that GM-CSF does not affect the number of days for resolution of fever or the hospitalisation period for this patients group, although a significant effect of GM-CSF was observed on neutrophil recovery.

\subsection{Introduction}

Chemotherapy-related neutropenia and fever is a frequently occurring complication during treatment of patients with cancer. In particular the severity and duration of neutropenia determines the risk of infection and the outcome of the patient (Bodey et al., 1966; Pizzo, 1984). Recently, different haematopoietic growth factors (HGFs) have been produced which has given the opportunity to modulate the period of granulocytopenia.

Two options are available for applying the HGFs after standard chemotherapy (Lieschke and Burgess, 1992). First, the HGF can be given as an adjunct to the chemotherapy till recovery of peripheral blood counts has been noted. In a number of studies this policy has resulted in a fifty percent reduction of infectious complications (Crawford et al., 1991; Gerhartz et al., 1993), while in other studies no significant effect has been shown with regard to the incidence of bacteraemia, days of fever, or the hospitalisation duration (Gorin et al., 1992; Pettengell et al., 1992). A second possibility would be to apply the HGF only in the case of infection (Biesma et al., 1990; Riikonen et al., 1994; Maher et al., 1994; Mayordomo et al., 1993). This approach would reduce the number of patients exposed to a growth factor and could potentially restrict the costs of treatment. A limited number of studies has tested this approach. Riikonen et al. (1994) demonstrated in a randomised study with 58 children that the application of GM-CSF during fever and neutropenia resulted in a faster recovery of the granulocytic lineage and a shortening of the period of hospitalisation. A recent double blind study with G-CSF in febrile neutropenia demonstrated that the greatest benefit was obtained in patients with a documented infection with a neutrophil count less than $0.1 \times 10^{9} / 1$ at start of G-CSF administration (Maher et al., 1994). 
We describe a prospective multicentre clinical trial which was designed to compare the effects of GM-CSF versus placebo in 153 adult patients with chemotherapy-related febrile neutropenia focused on whether GM-CSF affects the duration of hospitalisation in conjunction with quality of life and cost-effectiveness analysis.

\subsection{Patients and methods}

\subsubsection{Study population}

The study was carried out at the Departments of Haematology and/or Oncology of six University Hospitals and the Dr.Daniel den Hoed Cancer Centre from September 1991 till September 1994. Eligible patients with chemotherapy-related neutropenia $\left(<0.5 \times 10^{9} / 1\right)$ and fever (temperature of $>38^{\circ} \mathrm{C}$ over a $12 \mathrm{hr}$ observation period or $>38.5^{\circ} \mathrm{C}$ once) were entered. Patients with severe heart, lung, and liver impairment World Health Organisation (WHO) grade 3-4 were excluded as were patients with acute myeloid leukaemia, myelodysplasia or autologous or allogeneic bone marrow transplantation as well as patients already receiving antibiotics for the suspected infection. The study protocol was approved by the Medical Ethical Committees of the different hospitals and all patients gave informed consent.

\subsubsection{Design and treatment}

The study was a double-blind randomised phase III study. Patients were stratified for solid or haematological tumours and for the hospital. Enrolled patients were admitted to hospital and a full medical history was taken and physical examination was performed. Baseline investigations included full blood cell counts with differential white cell counts and sinus and chest radiographs. Cultures of blood (in duplicate), urine, and other suspicious sites were collected. In addition, serum was collected and frozen $\left(-80^{\circ} \mathrm{C}\right)$ at day 0 and 1 for cytokine analysis. GM-CSF or placebo $(5 \mu \mathrm{g} / \mathrm{kg} /$ day) was administered once daily subcutaneously for a minimum of 4 days and maximum of 14 days. GM-CSF or placebo was started simultaneously with intravenous empiric antibiotics according to a standardised local hospital policy. Both antibiotics and GM-CSF/placebo were discontinued if both the temperature normalised $\left(<37.5^{\circ} \mathrm{C}\right)$ and the granulocyte count was $1.0 \times 10^{9} / 1$ or more for two consecutive days. However, in the case of a leucocyte count of $10 \times 10 \%$ or more, the application of GM-CSF/placebo was stopped while the antibiotic treatment was continued until the temperature was normalised for two consecutive days. 
The patient was then observed for a $24 \mathrm{hr}$ period and if no sign of infection was noted the patient was discharged from the hospital and followed twice weekly for a period of 2 weeks or until the next chemotherapy course.

Lyophilised E.coli GM-CSF (Leucomax ${ }^{\otimes}$; kindly provided by Sandoz Pharma [Basle, Switzerland/Schering-Plough Kenilworth, NJ]) in vials of $400 \mu \mathrm{g}$ protein/vial was reconstituted in $1 \mathrm{ml}$ water. A vial identical in appearance to the active drug, but containing lyophilised placebo was also provided by Sandoz and used in a similar fashion.

\subsubsection{Study end points}

Primary end point was hospitalisation time with GM-CSF or placebo, which comprises the period of resolution of neutropenia $\left(>1.0 \times 10^{9} / 1\right)$ and fever defined as a temperature $<37.5^{\circ} \mathrm{C}$ for 2 consecutive days, followed by a $24 \mathrm{hr}$ observation period. For this reason blood and differential counts were performed daily and temperature was recorded 3 times a day. Secondary end points were days on antibiotics and incidence of change in antibiotic treatment. Inpatients were allowed to enter the study. Some patients remained in the hospital after completing the study for reasons other than continued morbidity related to fever and neutropenia. In these cases the hospitalisation duration was the study duration.

\subsubsection{Quality of life analysis}

The quality of life of the patients was scored by means of written questionnaires, which were filled in by the patients one day after stopping the GM-CSF or placebo treatment.

Based on methodological criteria concerning cost-effectiveness studies in cancer trials, the patient questionnaire consisted of a generic health status measurement instrument, a cancer-specific instrument and a valuation instrument (Bonsel et al., 1993; Uyl-de Groot et al., 1994III). The Karnofsky Performance Index and the Nottingham Health Profile were included as generic instruments, the Rotterdam Symptom Checklist as cancerspecific instrument and the EuroQol as valuation instrument (Uyl-de Groot et al., 1994III; Hunt et al., 1986; De Haes et al., 1990; Van Hout and McDonnell, 1991).

The Nottingham Health Profile incorporates the dimensions mobility, emotional reactions, energy, social isolation, pain and sleep. For the general population the average score for all dimensions is less than 10 (Bonsel et al., 1992). The EuroQol consisted of a descrip- 
tive part and a valuation part (a rating scale). The descriptive part allows the calculation of utilities. The utility scores are based on the valuation for each health state of patients and of a representative panel of the population (Van Hout and McDonnell, 1991). The average EuroQol score for the general population is more than 90 (Bonsel et al, 1992). The Rotterdam Symptom Checklist has the potential to add illness or treatment-related items. In this study, questions were added on possible side-effects associated with GMCSF, namely constipation, painful joints, palpitation, rash/eczema and sweating/perspiring.

Furthermore, data forms were used to get daily information on patients' quality of life during the period of fever and neutropenia. These forms were filled in daily by nurses. The descriptive part of the EuroQol was used, allowing the calculation of utilities.

\subsubsection{Cost analysis}

The cost analysis was based on a detailed review of all activities concerning the treatment of patients with fever and neutropenia. These activities included days in hospital, consultations, laboratory services, diagnostics including imaging procedures, antibiotics and GM-CSF. The data were derived from all registry forms and from daily data forms. The latter included information on type of hospital ward and consultations.

For each of these cost data unit prices were determined reflecting the real use of resources. The year of study was 1992 ( 1 US $\$ \approx 1.8$ Dutch guilders). The patients stayed on wards for regular oncological care, regular haematological care, protected environment and intensive care. The costs of hospital days were split into direct and indirect costs. The direct costs concerned manpower (doctors, nurses, etc.) and materials (medical devices, supportive patient care etc.). The indirect costs were related to overheads. The cost of hospitalisation amounted to approximately US $\$ 290$ per day for regular oncological care, to US $\$ 350$ per day for regular haematological care, US\$ 536 for staying in a protected environment and US\$1223 for an intensive care ward.

The output of hospital laboratories in the Netherlands is measured in terms of a point system. A unit price is associated per point. The unit prices differ across types of laboratories. The unit prices per point varied from US $\$ 0.62$ (biochemistry and haematology laboratories) to US $\$ 2.81$ (virology tests). A routine test (including haemoglobin (Hb), haematocrit $(\mathrm{Ht})$, leucocytes and platelets) amounted to 5.75 points and costed US $\$ 3.58$. For the diagnostics the Dutch tariff system has been used as an approximation of unit 
costs. The drug prices used were wholesale prices. A $300 \mu \mathrm{g}$ vial GM-CSF costed US $\$$ 138 and a $400 \mu \mathrm{g}$ vial costed US\$184. (See Appendix A4.3 for a list of unit prices used in the study).

\subsubsection{Measurement of serum cytokine levels}

GM-CSF, Granulocyte-CSF (G-CSF), Interleukin-6 (IL-6), and Tumour Necrosis Factor$\alpha$ (TNF- $\alpha)$ serum levels were quantified using a human GM-CSF, G-CSF, IL-6, or TNF$\alpha$ enzyme-linked immunosorbent assay (Elisa) kit (R\&D, Cambridge, United Kingdom) as recommended by the manufacturer.

\subsubsection{Statistical analysis}

The generalised Wilcoxon test was chosen to test the difference in duration of hospitalisation between the two treatment groups. The estimated sample size was based on the $\mathrm{H}_{0}$ hypothesis that the median duration of hospitalisation is 11 days in both treatment groups vs the $\mathrm{H}_{1}$ hypothesis assuming a clinically relevant difference between the two treatment groups with respect to the duration of hospitalisation of 2 days. Using a significance level of 0.05 a sample size of 70 patients per group revealed a power calculation of at least $75 \%$.

For the comparison of the clinical characteristics a chi-square, two-tailed Fisher's exact test, Wilcoxon-Mann-Whitney test, or Students's t-test as appropriate was used.

It was a feature of the study to allow patients to enter more than once. This was the case in 11 patients ( $7 \mathrm{GM}$-CSF $v s 4$ placebo). The results were analysed according to intention to treat. All patients who fulfilled the entry criteria and received at least one dose of study medication were included in this analysis. In the case of premature discontinuation, death or major protocol violation for the main end point analysis, the patient was "right" censored at day 14, the "worst outcome event". Additionally a per protocol analysis was performed. In this analysis the statistical approach was to censor at the time of discontinuation in the case of a non-informative dropout. If not then the "worst case" or day 14 was taken as the censor. Cumulative rates for days on study, days of neutropenia, and days of fever were estimated by Kaplan-Meier methods. Groups were compared by the generalised Wilcoxon test. Data are reported as median survival and absolute ranges. 
The risk reduction for assigned study medication with the theoretical discharge from hospital as dependent variable was adjusted for influential baseline covariates, chosen by step-wise selection, by using a Cox proportional regression model. The covariates selected were more than 11 days after last chemotherapy, solid tumour, and fever of unknown origin (FUO). Only variables whose coefficients had $P$-values of less than 0.05 were included. Adjusted risk reductions are presented.

A two-sided probability level of 0.05 or less was considered to indicate statistical significance. All analyses were performed using Statistical Analysis System version 6.08 (SAS Institute, Cary, N.C.). The quality of life items are presented as mean scores. For the comparison between items the Mann-Whitney and the Chi-square tests were used. The cost of both groups were compared with the Mann-Whitney test (Glantz, 1989).

\subsection{Results}

\subsubsection{Patient characteristics}

One hundred and fifty-three patients were entered in the study (Table 10.1). Of these patients 74 received GM-CSF and 79 placebo. Nine patients in the GM-CSF group and 10 patients in the placebo group were excluded from analysis due to ineligibility of main entry criteria, e.g. temperature less than $38^{\circ} \mathrm{C}$ or granulocyte count more than $0.5 \times 10^{9} / 1$.

Table 10.1 Study population

\begin{tabular}{|c|c|c|c|}
\hline No. & & $\begin{array}{r}\text { GM-CSF } \\
\text { group }\end{array}$ & $\begin{array}{r}\text { Placebo } \\
\text { group }\end{array}$ \\
\hline 153 & patients randomised & 74 & 79 \\
\hline$\downarrow \searrow$ & 19 ineligibility for entry criteria & 9 & 10 \\
\hline 134 & patients available for intention to treat analysis & 65 & 69 \\
\hline \multirow[t]{5}{*}{$\downarrow$} & 11 withdrawals before end of follow-up: & & \\
\hline & $\begin{array}{l}\text { incorrect treatment with study } \\
\text { medication or concomitant medication }\end{array}$ & 2 & 3 \\
\hline & possibility of side-effects & 1 & 2 \\
\hline & withdrawal consent & 1 & 1 \\
\hline & - adverse event & 1 & 0 \\
\hline 123 & patients available for per protocol analysis & 60 & 63 \\
\hline
\end{tabular}


Eleven patients (five GM-CSF, six placebo) were withdrawn before study completion and were excluded for the per protocol analysis. The reasons for excluding these patients were: incorrect treatment with study medication or concomitant medication $(n=5)$, adverse event $(n=1)$, suspected side-effects of treatment $(n=3)$, and refusal of further treatment $(n=2)$.

The baseline characteristics of the 134 patients are summarised in Table 10.2. The two treatment groups were well matched with regard to age, sex, tumour type and neutrophil count. The preceding chemotherapy in the GM-CSF group consisted of a cisplatin containing $(21 \%)$, anthracycline containing $(66 \%)$, or a high-dose Ara-C containing regimen $(10 \%)$. In the placebo group comparable treatment schedules were applied: $27 \%$ cisplatin containing, $57 \%$ anthracycline containing, and $2 \%$ a high-dose Ara-C containing regimen.

Table 10.2 Baseline characteristics of the study patients

\begin{tabular}{|c|c|c|}
\hline & $\begin{array}{r}\text { GM-CSF } \\
\text { group } \\
(n=65)\end{array}$ & $\begin{array}{r}\text { Placebo } \\
\text { group } \\
(n=69)\end{array}$ \\
\hline Median age (years, range) & $49(19-73)$ & $48(16-70)$ \\
\hline Male sex, $\mathrm{n}(\%)$ & $32(49)$ & $36(52)$ \\
\hline Tumour type, n (\%) & & \\
\hline$-\quad$ solid & $41(63)$ & $46(66)$ \\
\hline - lymphoma & $18(27)$ & $21(30)$ \\
\hline - $\quad A L L$ & $6(9)$ & $2(3)$ \\
\hline Days since last chemotherapy & 12 & 13 \\
\hline Baseline leucocyte count, n (\%) & & \\
\hline$-\quad<0.1 \times 10^{9} / 1$ & $42(64)$ & $39(56)$ \\
\hline$-\quad 0.1-0.5 \times 10^{9} / 1$ & $23(35)$ & $30(43)$ \\
\hline Baseline neutrophil count, n (\%) & & \\
\hline$-\quad<0.1 \times 10^{9} / 1$ & $42(64)$ & $39(65)$ \\
\hline$\quad 0.1-0.5 \times 10^{9} / 1$ & $16(24)$ & $21(30)$ \\
\hline Microbiologically or clinical documented infection, $n(\%)$ & $45(69)$ & $42(61)$ \\
\hline - $\quad$ culture positive with bacteraemia & $12(18)$ & $13(18)$ \\
\hline - $\quad$ culture positive without bacteraemia & $24(36)$ & $19(27)$ \\
\hline - $\quad$ culture negative but clinically documented & $9(13)$ & $10(14)$ \\
\hline
\end{tabular}


With regard to the microbiologically or clinically documented infection no significant difference was observed for fever of unknown origin, bacteraemia and culture positive infection. The positive blood cultures consisted in $44 \%$ of gram-positive bacteria and in $56 \%$ of gram-negative bacteria in the GM-CSF group. In the placebo group $50 \%$ of the cultures contained gram-positive and $50 \%$ gram-negative bacteria.

\subsubsection{Neutropenia and fever}

GM-CSF enhanced the neutrophil recovery as depicted in Figure 10.1. A significant difference in absolute neutrophil count was observed at days 4 and $5(P<0.001)$. A difference was also observed for the numbers of monocytes and eosinophils at last day of GM-CSF/placebo treatment in 100 evaluable patients. In the GM-CSF group the median number of monocytes was $0.9 \times 10^{9} / 1$ (range 0.0 .4 ) and of esinophils $0.5 \times 10^{9} / 1$ (range 0 1.2). In the placebo group these values were $1.2 \times 10^{9} / 1$ (range $0.1-4.9 ; P<0.03$ ) and $0.3 \times 10^{9} / 1(0.0-0.5 ; P=0.45)$, respectively. However, despite the enhancing effect of GMCSF on the neutrophil recovery, no differences were observed in the median days of neutrophils less than $0.5 \times 10^{9} / 1$ and $1.0 \times 10^{9} / 1$ (Table 10.3).

Figure 10.1 Neutrophil recovery, temperature curve and patient number during GM-CSF or placebo treatment

Figure 10.1A Neutrophil recovery

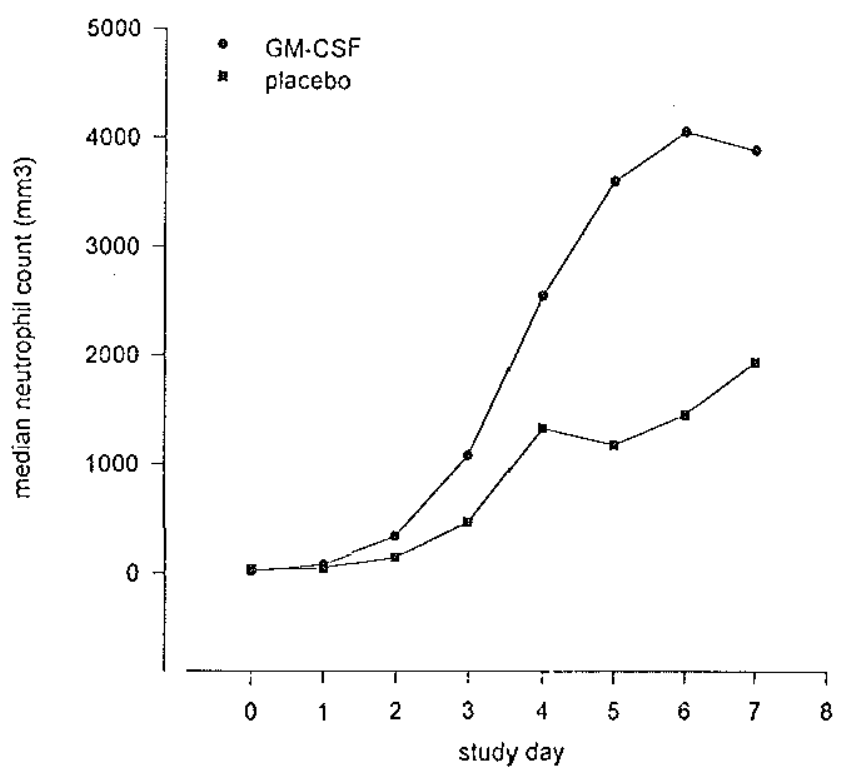




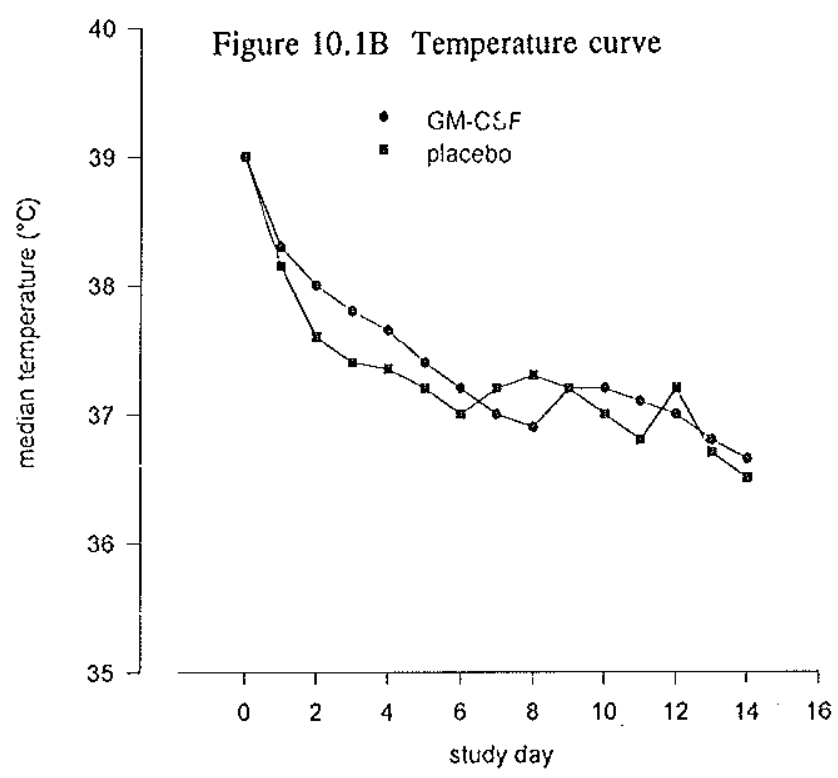

Figure 10.1C Patient number during the treatment of GM-CSF or placebo

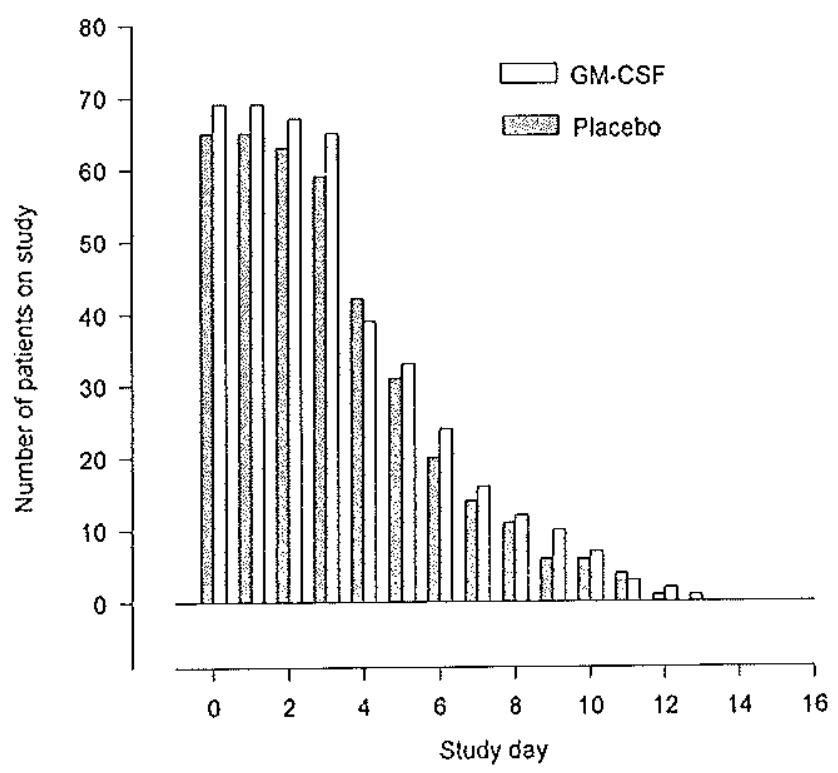

No difference was observed in time to resolution of fever. The median temperature curve was higher in the GM-CSF group compared to the placebo group during the first 6 days of treatment with a significant difference at day $2(P<0.05$, Figure 10.1). 
Table 10.3 Days of neutropenia and fever and days on study (hospitalisation) per treatment group $(n=134)$

\begin{tabular}{|l|r|r|r|r|l|}
\hline & \multicolumn{2}{|c|}{ GM-CSF group } & \multicolumn{2}{|c|}{ Placebo group } & \\
\hline & median & range & median & range & $P$-value \\
\hline ANC $<0.5 \times 10^{9} / 1$ (days) & 3 & $1-14$ & 4 & $1-14$ & $\mathrm{NS}$ \\
ANC $<1.0 \times 10^{9} / 1$ (days) & 4 & $1-14$ & 4 & $1-14$ & $\mathrm{NS}$ \\
ANC at day $4\left(\times 10^{9} / 1\right)$ & 2.9 & $0-25$ & 1.3 & $0-9$ & $<0.005$ \\
Fever (days) & 3 & $1-14$ & 3 & $1-14$ & $\mathrm{NS}$ \\
Days of hospitalisation & 6 & $3-14$ & 7 & $4-14$ & $\mathrm{NS}$ \\
\hline
\end{tabular}

NS: not significant; ANC: absolute neutrophil count.

The estimated median survival for the primary end point of the study (Figure 10.2), e.g. normalisation of temperature and a granulocyte count of more than $1.0 \times 10^{9} / 1$ during $48 \mathrm{hr}$ or a leucocyte count of $10 \times 10^{9} / 1$ or more both followed by a $24 \mathrm{hr}$ observation period, was reached after a median of 6 days (range: 3-14) in the GM-CSF group and 7 days (range: $4-14 ; P=0.27$ ) in the placebo group according to the intention to treat analysis. Comparable results were obtained with the per protocol analysis: GM-CSF: 6 days (range: $3-14$ ); placebo: 6 days (range: $4-14 ; P=0.33$ ).

Figure 10.2 Estimated median survival for the primary end point of the study

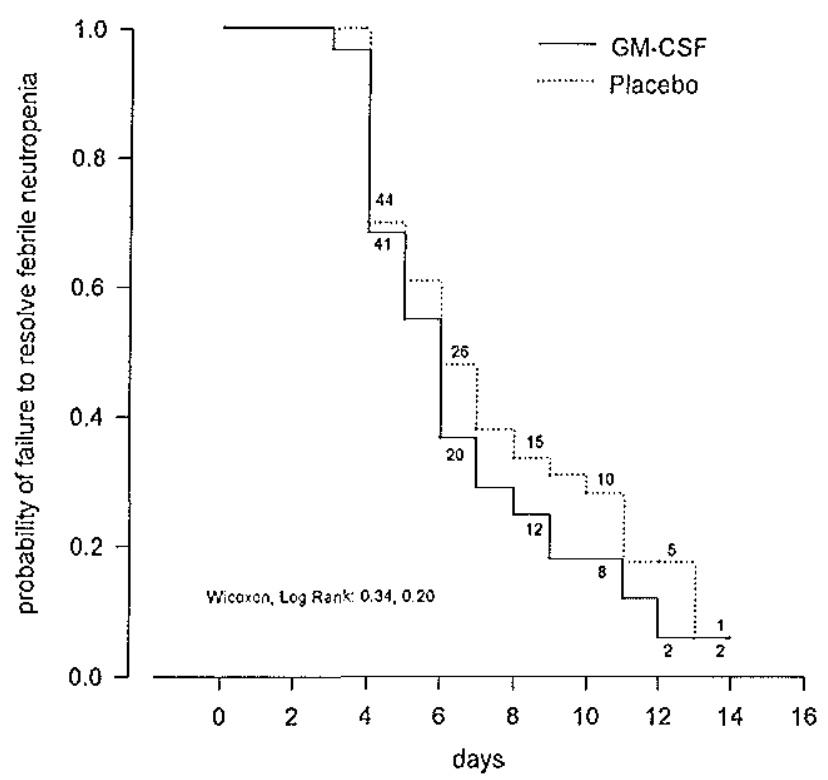


Finally, the proportion of patients in the GM-CSF and placebo arm was similar with regard to the hospitalisation period of more than 10 days; $9 \%$ in the GM-CSF group and $10 \%$ in the placebo group.

\subsubsection{Antibiotics and GM-CSF}

All patients were treated with intravenous antibiotics. In the GM-CSF group $15 \%$ received imipenem, $23 \%$ cefuroxime in combination with an aminoglycoside, $13 \%$ augmentin in combination with an aminoglycoside and $20 \%$ ceftazidime. In the placebo group the percentages were $11 \%, 14 \%, 23 \%$, and $18 \%$, respectively. Antibiotic treatment was changed in $29 \%$ of the GM-CSF group and $24 \%$ in the placebo group. Six (9\%) of the patients treated with GM-CSF and three $(4 \%)$ of the patients receiving placebo were given intravenous antifungal therapy. The median duration of GM-CSF and placebo application for the total group was 5 days in both the GM-CSF arm (range: 1-14) and placebo arm (range: 1-13). However, not in all cases the antibiotic treatment was stopped at the same day of GM-CSF/placebo cessation. In the GM-CSF arm 16 patients had a prolonged administration of antibiotic treatment between 3-5 days, and three patients between 5-10 days. In the placebo arm prolonged antibiotic administration was given in eight patients between 3-5 days and in two patients between 7-9 days.

\subsubsection{Subgroup, adjusted and multivariate analysis}

First, it was evaluated whether a difference in response was noticed in patients with baseline neutrophils $<0.1 \times 10^{9} / 1$ vs $0.1-0.5 \times 10^{9} /$. No difference was observed between both groups for time to reach a neutrophil count $>1.0 \times 10^{9} / 1$ in combination with time for resolution of fever. Secondly, Table 10.4 shows the result of a Cox proportional hazards regression analysis for the primary end point in which the difference between the two treatment regimens was estimated after adjustment for the independent associated covariates: more than 11 days since last chemotherapy, solid tumour, and fever of unknown origin. GM-CSF application did not influence the primary end point significantly, but a trend was observed. When all prognostic factors were taken into account simultaneously in the Cox regression model, the risk reduction of the primary end point due to GM-CSF treatment was $29 \%(P=0.12)$. The unadjusted risk reduction of GM-CSF treatment was $23 \%(P=0.20)$. 
10 GM-CSF in chemotherapy-related febrile neutropenia

Table 10.4 Analysis of the risk of hospitalisation in a Cox regression model

\begin{tabular}{|l|r|r|}
\hline Analysis & Risk reduction & $P$-value \\
\hline Unadjusted: GM-CSF treatment & $23 \%$ & 0.200 \\
& & \\
Multivariate (Cox regression model) & & \\
Days since last chemotherapy (>11 days) & $41 \%$ & 0.016 \\
Solid tumour & $55 \%$ & 0.001 \\
Fever of unknown origin & $42 \%$ & 0.016 \\
& & \\
Adjusted: GM-CSF treatment & $29 \%$ & 0.120 \\
\hline
\end{tabular}

\subsubsection{Supportive care}

The median number of red blood cell transfusion was similar in the GM-CSF group versus the placebo group, 2 (range: $0-8$ ) vs 2 (range: $0-8$ ), respectively $(P=0.50$ ). Platelet transfusion was given in a median of 0 patients (range: $0-5$ ) in the GM-CSF group and in 0 patients (range: $0-4)$ in the placebo group $(P=0.72)$.

\subsubsection{Results quality of life analysis}

The results of the quality of life analysis are based on 113 daily data forms and 90 quality of life questionnaires. The discrepancy between the numbers of questionnaires and data forms is due to a later start of the cost-effectiveness study. The questionnaires and data forms that are not included are centre related, so it is very unlikely that as a consequence of this a bias would have been introduced.

The results of the patient questionnaires are summarised in Table 10.5. The scores of the Karnofsky Performance Index were greater among the placebo group, i.e. a mean value of 63 in the GM-CSF group versus 73 in the placebo group $(P<0.05)$. The patients in the placebo group reported less complaints on the Nottingham Health Profile than the patients who received GM-CSF treatment. The mobility, emotional, and energy problems were less pronounced in the placebo group than in the GM-CSF group $(P<0.05)$. No significant differences were noticed in the EuroQol scores using patient values. The population value was in favour of the placebo group, namely 54 in the GM-CSF arm and 66 in the placebo arm $(P<0.05)$. 
Table 10.5 Mean scores of quality of life in both treatment arms

\begin{tabular}{|c|c|c|c|}
\hline & $\begin{array}{r}\text { GM-CSF group } \\
(n=46)\end{array}$ & $\begin{array}{r}\text { Placebo group } \\
\qquad(n=44)\end{array}$ & $P$-value \\
\hline Karnofsky Index ${ }^{1}$ & 63 & 73 & 0.034 \\
\hline Nottingham Health Profile ${ }^{2}$ & & & \\
\hline - Mobility & 30 & 16 & 0.009 \\
\hline - Emotional reactions & 20 & 9 & 0.008 \\
\hline - Energy & 57 & 36 & 0.014 \\
\hline - Social isolation & 11 & 5 & 0.128 \\
\hline - Pain & 23 & 19 & 0.151 \\
\hline - Sleep & 35 & 24 & 0.116 \\
\hline EuroQol & & & \\
\hline - Patient score & 55 & 57 & 0.760 \\
\hline - Population score ${ }^{3}$ & 54 & 66 & 0.025 \\
\hline Most importamt symptoms/complaints ${ }^{4}$ & & & \\
\hline 1. Fatigue & 2.9 & 2.5 & 0.108 \\
\hline 2. Lack of appetite & 2.7 & 2.0 & 0.006 \\
\hline 3. Lack of energy & 2.5 & 1.9 & 0.003 \\
\hline 4. Dry mouth & 2.4 & 1.9 & 0.161 \\
\hline 5. Sweating, perspiring & 2.2 & 1.9 & 0.555 \\
\hline 6. Sore mouth / pain when swallowing & 2.1 & 2.0 & 0.776 \\
\hline
\end{tabular}

${ }^{1}$ Range $0-100$ : from worst $(0)$ to best health states $(100) ;{ }^{2}$ Range 100-0: from worst $(100)$ to best health states $(0) ;{ }^{3}$ Utility scores based on the valuations of patients and of a representative panel of the population; ${ }^{4}$ Answer possibilities: $1=$ not at all, $2=$ a little, $3=$ quite a bit and $4=$ very much. The average values are presented.

The most important complaints and symptoms reported on the Rotterdam Symptom Checklist were tiredness, lack of appetite, lack of energy, dry mouth, sweating/perspiring and sore mouth and/or pain when swallowing. Patients in the placebo group experienced less problems concerning appetite and energy than the patients in the GM-CSF group $(P<0.01)$. Regarding tiredness, dry mouth and sweating no difference was observed. 
The results of the population scores (data forms) derived from the descriptive part of the EuroQol are presented in Figure 10.3. Patients receiving placebo treatment were feeling better during the hospitalisation period than the patients in the GM-CSF group. From day 8 , the observations are biased as many patients were already discharged from hospital.

Figure 10.3 EuroQol population scores in the GM-CSF and in the placebo group

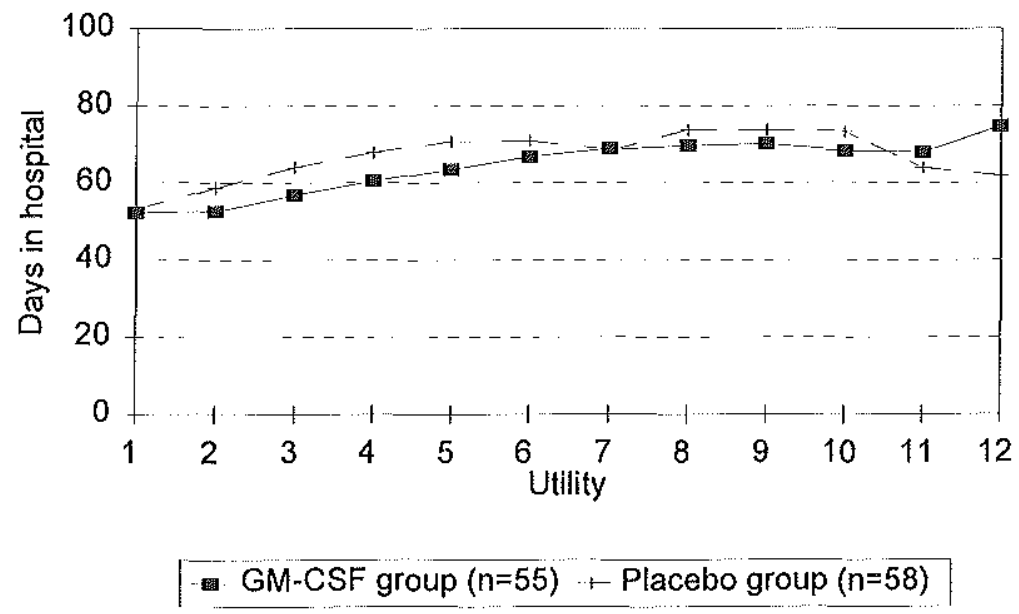

\subsubsection{Results cost analysis}

The median number of days in hospital in the GM-CSF group was 6 days (mean: 7.25). The hospital care was classified as follows: $87 \%$ regular oncological or haematological care, $11 \%$ protected environment care and $2 \%$ intensive care. Patients treated with placebo had a median hospital stay of 7 days (mean 8.33). This group was divided into: $86 \%$ regular oncological or haematological care, $13 \%$ care in a protected environment and $1 \%$ intensive care.

The median cost of hospitalisation in the GM-CSF group was US $\$ 2130$ (range: 8606420 ) and in the placebo group US $\$ 2450$ (range: 1140-7130). The median cost of antibiotics amounted to US\$ 630 (range: 130-3790) in the GM-CSF group and to US\$ 580 (range: 144-2930) in the placebo group. The cost of GM-CSF was US\$ 1100 (range: 280-3860). Additional costs included laboratory services, medical procedures, consultations and blood transfusions. The median costs were equal in both arms, namely US $\$$ 470 (range: $120-2830$ in the GM-CSF group and range: 170-2680 in the placebo group). The median of all costs was US $\$ 4140$ (mean: 5180 ; range: 1710-14650) in the GM-CSF group and US $\$ 3590$ (mean: 4180; range: $1680-10990)$ in the placebo group $(P<0.05)$. 


\subsubsection{Adverse events}

The events observed during the treatment period are reported in Table 10.6. A great variety in events was observed. Myalgia and/or rash and/or bone pain and/or oedema was noticed in $20 \%$ of the GM-CSF treated patients and $6 \%$ in the placebo treated patients $(P=\mathrm{NS})$. No difference was observed in the mortality rate between both groups. In the GM-CSF arm one patient died due to acute respiratory distress syndrome. In the placebo arm two patients died as a result of pulmonary embolism.

Table 10.6 Major medical events reported during the treatment of antibiotics plus GM-CSF or placebo

\begin{tabular}{|l|r|r|}
\hline & GM-CSF group & Placebo group \\
\hline Gastro-intestinal & $35 \%$ & $23 \%$ \\
Cardiac dysfunction & $6 \%$ & $1 \%$ \\
Biochemical abnormalities & $30 \%$ & $27 \%$ \\
Thrombosis & $3 \%$ & $0 \%$ \\
Myalgia & $4 \%$ & $1 \%$ \\
Rash & $4 \%$ & $0 \%$ \\
Bone pain & $1 \%$ & $1 \%$ \\
Oedema & $12 \%$ & $5 \%$ \\
Death & $1 \%$ & $2 \%$ \\
\hline
\end{tabular}

\subsubsection{Cytokine analyses}

Serum cytokine levels were measured at presentation and $24 \mathrm{hr}$ after initiation of therapy in 60 patients (28 GM-CSF treated patients and 32 placebo treated patients; Table 10.7). The analysis concerned eight patients with bacteraemia, 25 patients with a clinically documented infection and 27 patients with fever of unknown origin. At presentation high levels of Interleukin-6 (IL-6) and G-CSF were observed especially in the patients with bacteraemia. During the first $24 \mathrm{hr}$ of treatment no change in the cytokine profile was observed due to the administration of GM-CSF, except for significantly higher levels of GM-CSF that were measured in the GM-CSF treated group (Table 10.7). 
10 GM-CSF in chemotherapy-related febrile neutropenia

Table 10.7 Cytokine profiles on day 0 and 1 of treatment

\begin{tabular}{|c|c|c|c|c|c|c|c|}
\hline \multirow[t]{2}{*}{ Treatment arm } & \multicolumn{3}{|c|}{ GM-CSF group } & \multicolumn{4}{|c|}{ Placebo group } \\
\hline & day 0 & & day 1 & & day 0 & & ay 1 \\
\hline GM-CSF & $28 \pm$ & $709 \pm$ & $126^{*}$ & $13.8 \pm$ & 3.9 & $54 \pm$ & 37 \\
\hline G-CSF & $3459 \pm 583$ & $2858 \pm$ & 604 & $2897 \pm$ & 498 & $2394 \pm$ & 512 \\
\hline TNF $-\alpha$ & $1.0 \pm 0.43$ & $0.18 \pm$ & 0.77 & $2.0 \pm$ & 1.2 & $1.25 \pm$ & 0.9 \\
\hline IL -6 & $304 \pm$ & $238 \pm$ & 57 & $324 \pm$ & 64 & $262 \pm$ & 61 \\
\hline
\end{tabular}

Cytokine serum levels of G-CSF (mean $x \pm S E$ (standard error)), pg/ml; GM-CSF $(x \pm S E)$ $\mathrm{pg} / \mathrm{ml}$; Tumour Necrosis Factor- $\alpha$ (TNF- $\alpha), \mathrm{x} \pm \mathrm{SE} \mathrm{ng} / \mathrm{ml}$ and Interleukin-6 (IL-6), $\mathrm{x} \pm \mathrm{SE}$ $\mathrm{pg} / \mathrm{ml} ;$ " A significant difference was observed at day 1 of treatment $(P<0.0001)$.

\subsection{Discussion}

The present study demonstrates that GM-CSF accelerates the neutrophil recovery in patients who received antibiotics for febrile neutropenia after chemotherapy. Enhancement of the neutrophil recovery by GM-CSF was irrespective of whether patients presented with baseline granulocytes less than $0.1 \times 10^{9} / 1$ or between $0.1-0.5 \times 10^{9} / 1$. However, the enhancing effect of GM-CSF on the neutrophil recovery did not occur immediately, but was observed only 4 and 5 days after start of treatment.

Despite the faster neutrophil recovery in the GM-CSF group no difference was observed in the resolution of fever between the GM-CSF and placebo group. Previous studies demonstrated distinct correlation between resolution of fever and neutrophil recovery (Bodey et al., 1966). However, these studies were conducted almost entirely in patients with a long-lasting neutropenia (Pizzo et al., 1982). The observations apply particularly to patients with acute leukaemia who are treated with intensive chemotherapy. The present study and other studies in cancer patients show that this correlation is less prominent for patients treated with less intensive chemotherapy regimens. In these patients the febrile period is usually short and depends on the response to antibiotics. In the placebo group the median duration for resolution of fever $\left(<37.5^{\circ} \mathrm{C}\right)$ was 3 days, while a granulocyte count of more than $1 \times 10^{9} / 1$ was reached after a median of 4 days. A possibility in the GM-CSF group could be that GM-CSF negatively affected the days for resolution of fever. Phase I-II studies have demonstrated that GM-CSF might induce fever. However, this was observed especially in patients treated with a higher dose of GM-CSF (Lieschke and Burgess, 1992; Hazenberg et al., 1989). A recent study with G-CSF in febrile neutropenia demonstrated that G-CSF hastened the neutrophil recovery, but did not affect 
the duration of fever (Maher et al., 1994). Since the period of fever and severe granulocytopenia was not reduced by GM-CSF treatment, no difference was observed in the duration of hospitalisation. This lack of difference in hospitalisation duration between both arms resulted that GM-CSF was not cost-effective for the total group of patients with chemotherapy-related febrile neutropenia. Treatment costs were significantly higher than those in the placebo group, mainly due to the costs of GM-CSF. In part this is caused by the fact that GM-CSF is only available in vials of $300 \mu \mathrm{g}$ and $400 \mu \mathrm{g}$. In this study, the GM-CSF was administered at a dose of $5.0 \mu \mathrm{g} / \mathrm{kg} /$ day, implying that a patient of $65 \mathrm{~kg}$ received $325 \mu \mathrm{g} \mathrm{GM-CSF}$. Correcting for this loss of GM-CSF or applying a $300 \mu \mathrm{g}$ vial only would reduce the costs of GM-CSF by approximately ten percent.

Adverse events were frequently noticed in this study and can be ascribed to the underlying disorder and treatment. Quality of life analyses demonstrated significant differences in favour of the placebo group. However, no increased mortality was observed in the GMCSF treated group.

Different cytokines were measured to analyse whether the GM-CSF application might change the IL-6, G-CSF, and TNF- $\alpha$ serum levels during the treatment. Elevated levels were measured, but GM-CSF did not modulate these cytokine profiles significantly. Recent studies in mice with non-compromised bone marrow have demonstrated that GMCSF priming is associated with an enhanced production of cytokines after challenge with endotoxin, resulting in an increased mortality (Tiegs et al., 1994). The present study revealed no major changes in the cytokine profile during the administration of GM-CSF.

Four studies have been conducted in patients with febrile neutropenia (Biesma et al, 1990; Riikonen et al., 1994; Maher et al., 1994; Mayordomo et al., 1993). In three studies a significant advantage of GM-CSF or G-CSF treatment was observed (Riikonen et al., 1994; Maher et al., 1994; Mayordomo et al., 1993). In the present study only a trend for advantage of GM-CSF application is seen. The difference might be ascribed to differences in patients categories and treatment protocols. This is supported by the multivariate analysis in which tumour type was an independent prognostic factor for the success rate of treatment. Furthermore, a remarkable difference is observed in duration of antibiotic application between the different studies which seems to be the most relevant factor for the hospitalisation period. In the study of Riikonen et al. (1994) antibiotic treatment was continued for a minimum of 5 days while in the study of Maher et al. (1994) antibiotic treatment was continued for 4 days after normalisation of temperature. In the present study the antibiotic treatment was intended to stop after 2 consecutive days with resolution of fever and granulocytopenia. The differences in antibiotic policy have a great 
impact on the hospitalisation period. The median number of days in hospital for the GMCSF and G-CSF arm of the studies of Riikonen and Maher were 9 and 8 days, respectively. In the present study the median hospitalisation duration while on study was 6 days in the GM-CSF arm and 7 days in the placebo arm.

The results in this study do not exclude that a subgroup of patients, e.g. patients with a hospitalisation period longer than 10 days and neutropenia might benefit from the application of GM-CSF. In both previous studies (Riikonen et al., 1994; Maher et al., 1994) in febrile neutropenia a significant advantage of HGF application was observed, resulting in a significant reduction in hospitalisation duration. However, in the present study the follow-up period was only 14 days and prolonged hospitalisation was only observed in $10 \%$ of the patients which makes further analyses inadequate. Finally, the dose of GM-CSF used in this study seems to be adequate. A distinct effect on the neutrophil recovery was observed. Moreover, the results obtained for duration of severe neutropenia were comparable with the G-CSF study, in which a dose of $12 \mu \mathrm{g} / \mathrm{kg} /$ day was used (Maher et al., 1994).

In conclusion the here presented study demonstrates that the application of GM-CSF in febrile neutropenia did not result in a significant shortening of the hospitalisation period, despite a faster recovery of the neutrophils for the whole group of patients. 


\section{Part IIV}

Implementation of new technologies 

Costs of introducing ABMT in the Netherlands

\subsection{Summary}

In a retrospective study we calculated the costs of introducing ABMT in the treatment of patients with malignant lymphoma and acute leukaemia in the Netherlands. The cost analysis has been performed in five university hospitals and one cancer centre, in a series of patients with intermediate- and high-grade non-Hodgkin's lymphoma (NHL) and patients with acute myeloid leukaemia (AML). Conventional treatment consisted of chemotherapy. The average costs of the conventional NHL treatment varied from US\$ 3120 to US $\$ 12900$. The costs of ABMT amounted to US $\$ 40220$. In the AML group the costs of conventional treatment amounted to about US\$ 11040 , as only $50 \%$ of the patients were treated further. The costs of ABMT, including a follow-up period of two years, amounted to US\$55440. In the Netherlands the total number of ABMTs per year in these patient groups was estimated at 230; 180 in the NHL group and 50 in the AML group. The costs of introducing ABMT in the NHL group will vary between 4.93 and 6.68 million dollars and for the AML group these costs were estimated at 2.22 million dollars. As a result, the total extra costs of introducing ABMTs are expected to be between 7.15 and 8.90 million dollars.

\subsection{Introduction}

Over the past decade ABMT in combination with high-dose chemotherapy has become a widely applied treatment for certain patients with haematological malignancies. The number of ABMTs actually carried out is increasing per year (Singer and Goldstone, 1986; Gorin et al., 1984; Dufoir et al., 1992; Desch et al., 1992). ABMT is currently performed more frequently than heart transplantation (Durbin, 1988). 
In the Netherlands, as in many European countries, little is known about the financial consequence of introducing ABMTs to the clinical setting (Durbin, 1988; Lobo et al., 1991). Furthermore, it has become increasingly apparent, that resources for the provision of health care are limited. The Dutch government has already provided an additional budget of 3.4 million dollars for ABMT in 11 haemato-intensive care hospitals. In this study, we considered the macroeconomic consequences of introducing the use of ABMT in the Netherlands in terms of the additional resources required to finance ABMT for patients with non-Hodgkin's lymphoma (NHL) and acute myeloid leukaemia (AML).

\section{$11.3 \quad$ Patients and methods}

\subsubsection{Study population}

The NHL group consisted of patients with NHL of intermediate- and high-grade malignancy. These patients had had a previous $\mathrm{CR}$ and thereafter a (first or second) relapse. They received two reinduction courses of chemotherapy and showed a partial or complete response. Data of 50 patients with NHL were examined; 25 patients had ABMT and 25 received conventional treatment.

Furthermore, we considered patients with AML in first and second remission. Patient and hospital records of 30 patients with AML were studied; 20 patients received ABMT and 10 conventional treatment.

\subsubsection{Treatment modalities}

A panel of haematologists defined current treatment practice and protocols of the patient groups mentioned above. The panel consisted of five haematologists from academic institutes with considerable experience with the treatment of lymphoma and acute leukaemia.

The conventional treatment of NHL patients differed across patients and centres. Chemotherapy regimens administered were, for example, the CHOP, ProMACE-MOPP, CAMP, DHAP and the IMVP regimen (Glossary of chemotherapy regimens: page 166) (Fisher et al., 1993; Armitage, 1993; Verdonck et al., 1992; Cooleman, 1985). We focused on patients receiving ProMACE-MOPP, DHAP, CHOP and IMVP regimens, as these regimens were most often used. 
Some regimens were given as outpatient schedules, other regimens were given on an inpatient basis. An abbreviated scheme of the treatment patterns for the NHL group is shown in Figure 11.1.

Figure 11.1: Model of non-Hodgkin's lymphoma treatment

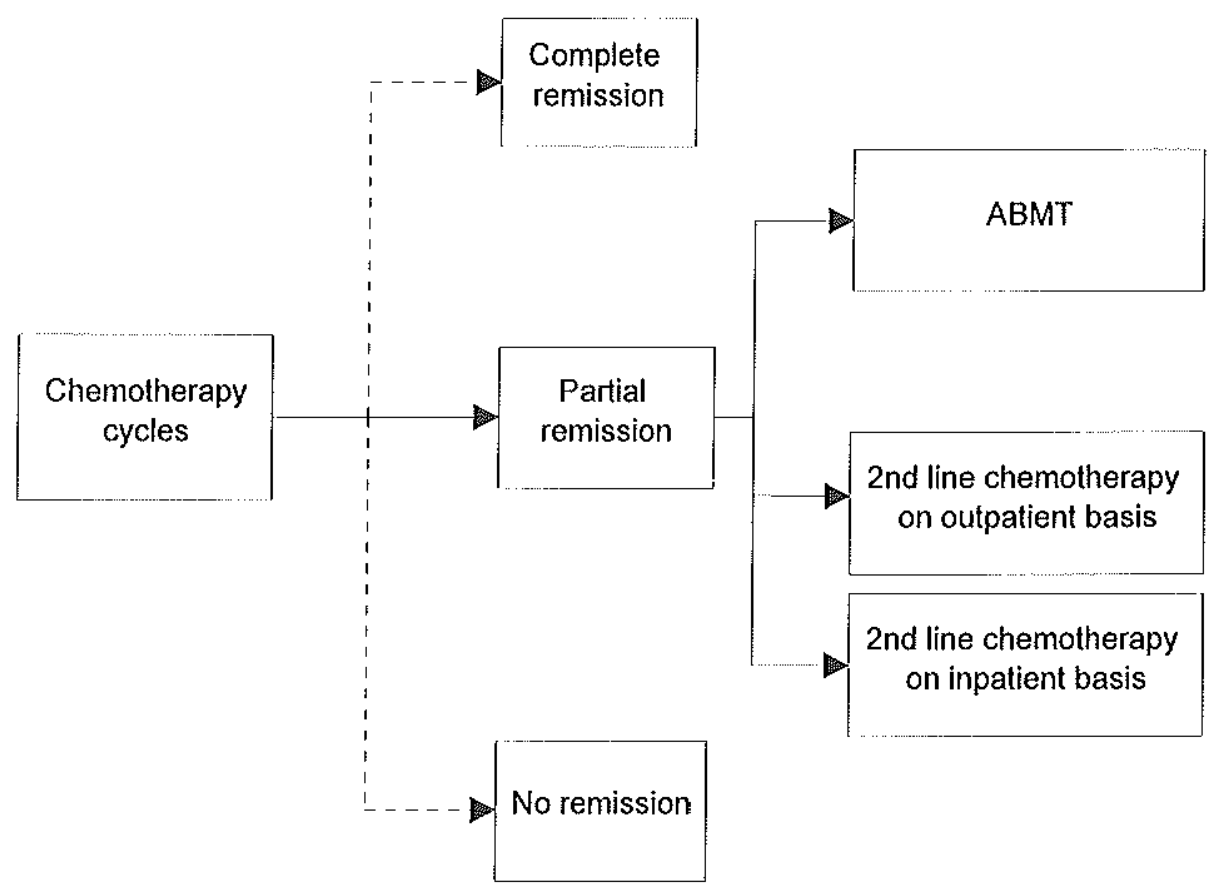

In the Netherlands, the alternative to ABMT in the AML group is "no further treatment", as according to a currently ongoing prospective randomised study. The best treatment option is still subject to debate. As the purpose of this study is to assess the costs of introducing ABMT in the Netherlands, we used the "no further treatment" option (= option 1) as the alternative to ABMT. Figure 11.2 shows an abbreviated scheme of the treatment patterns for the AML group. In the "no further treatment" arm, about $60 \%$ of the patients will relapse. For these patients, sometimes the decision was to try and reinduce a subsequent complete remission, or otherwise to withhold further therapy. In the past (situation with no ABMT), many patients did not receive any further therapy due to medical complications (about 50\%). The other patients received a relapse treatment. For the cost analysis, we considered a follow-up period of at the most two years. 
Figure 11.2 Model of treatment for patients with acute myeloid leukaemia

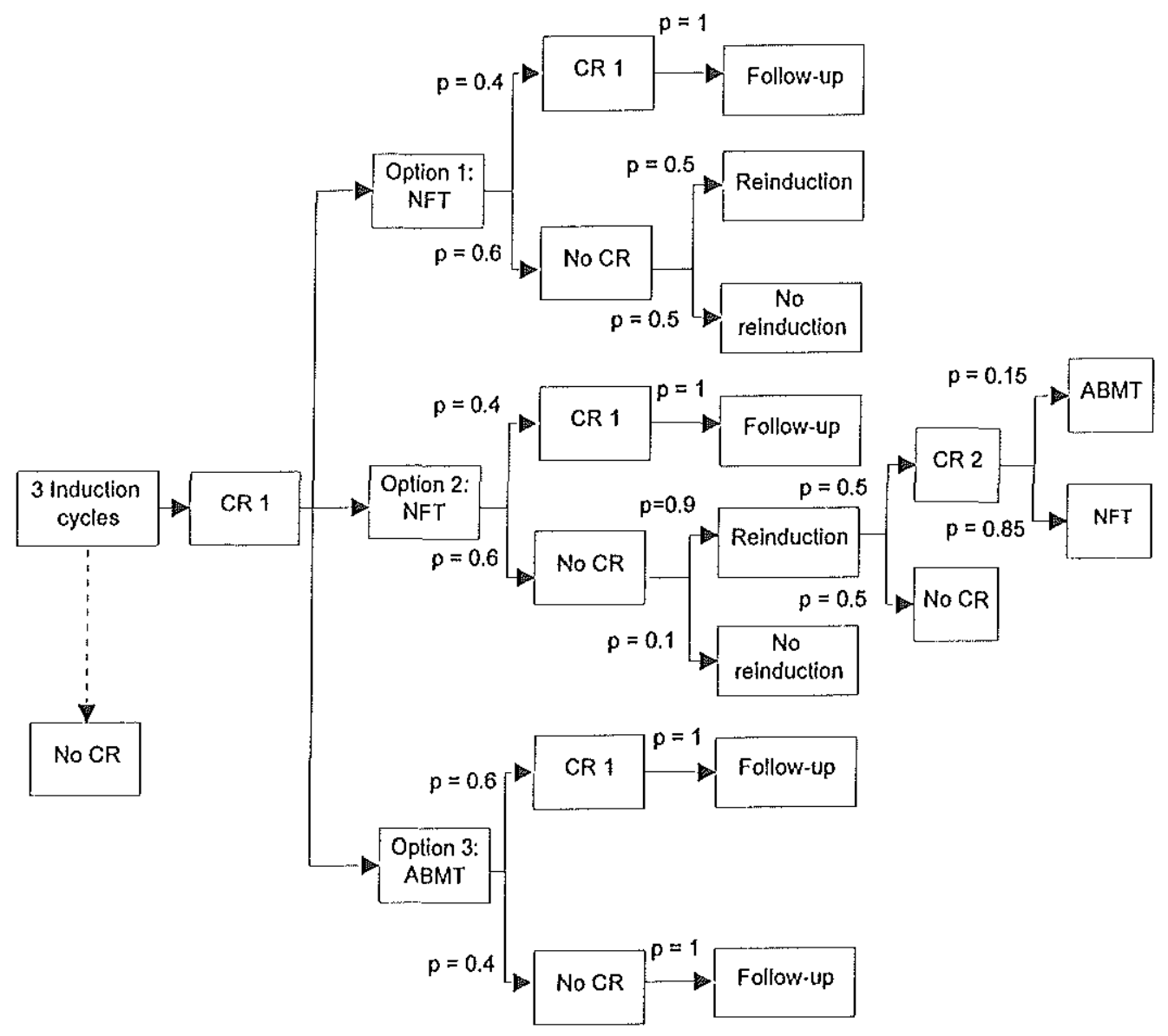

In the treatment of patients with AML in second CR, it is also possible to use ABMT. In general, bone marrow (BM) was harvested after first $\mathrm{CR}$ (= option 2 ). About $60 \%$ of the patients relapsed. These patients received at least 1 reinduction cycle. Of these patients, about $50 \%$ attained a second CR. According to the panel, about $15 \%$ of the AML patients in second remission would receive an ABMT. Option 3 describes the ABMT application in patients in first CR. 


\subsubsection{Cost analysis}

The cost analysis has been performed in five university hospitals and one cancer centre. The activities considered were days in hospital, outpatient visits, medical procedures (laboratory services, diagnostics), medication (cytostatics, antibiotics, antiemetics, etc.) and blood transfusions. These costs were registered through cost registry forms and reflect full costs including overheads. Cost price studies, measuring real resource use, have been carried out for all costs. The year of study was 1992 .

For the calculation of the total costs of introducing ABMT the expected number of ABMTs per year was also estimated. The incidence, remission and relapse rates per patient group have therefore been collected. These rates are derived from existing registration systems and from the Dutch Working Party on Haemato-Oncology (Medical Insurance Board, 1993). The extra costs of introducing ABMT is calculated by subtracting the costs of conventional therapy from the treatment costs of the strategy with ABMT.

\subsection{Results}

\subsubsection{Treatment cost NHL group}

Depending on the chemotherapy regimen the patients were hospitalised or they were treated on an outpatient basis. Chemotherapy regimens administered on an outpatient basis were CHOP and ProMACE-MOPP. The costs are summarised in Table 11.1. CHOP therapy was the cheapest chemotherapy. The average costs of CHOP chemotherapy amounted to about US\$3120. Patients who failed CHOP received ProMACE-MOPP, DHAP or IMVP regimens. The patients who were treated with DHAP and IMVP regimens were hospitalised. Per chemotherapy cycle, patients spent 5-7 days in the hospital and received an average of four cycles. The most expensive treatment was estimated at about US\$ 12900 . 
Table 11.1 Average costs of chemotherapy treatments in NHL

\begin{tabular}{|l|r|r|}
\hline & $\begin{array}{r}\text { Costs of outpatient } \\
\text { administration } \\
\text { (US\$) }\end{array}$ & $\begin{array}{r}\text { Costs of inpatient } \\
\text { administration } \\
\text { (US\$) }\end{array}$ \\
\hline Regimen 1: & CHOP therapy & DHAP therapy \\
- Hospitalisation / outpatient visits & 1070 & 7088 \\
- Medical procedures & 1355 \\
- Medication & 695 & 1740 \\
Total & 3120 & 3888 \\
& & 12716 \\
Regimen 2: & ProMACE-MOPP therapy & IMVP therapy \\
- Hospitalisation / outpatient visits & 1605 & 9924 \\
- Medical procedures & 1665 & 1476 \\
- Medication & 695 & 1500 \\
Total & 3965 & 12900 \\
\hline
\end{tabular}

Concerning ABMT, a pre-transplantation and a transplantation period were distinguished. An additional study was performed to assess the average cost of BM harvest, including cryopreservation and BM cultures. This cost was estimated at US $\$ 2042$, subdivided into personnel US $\$ 689$, operating room US\$ 543 , medical equipment US $\$ 436$, materials US\$ 222 and quality control of the BM US\$152. BM was unpurged. The total expenses during the pre-transplantation period were about US $\$ 5480$. However, only $67 \%$ of the bone marrow harvests actually resulted in a transplantation. Therefore, the mean costs of the pre-transplantation period amounted to US\$8220 ( $=1.5 \mathrm{x}$ US\$ 5480). The transplantation period costed about US\$ 32000 (range: US\$23960 - US\$ 69100); hospitalisation was responsible for $60 \%$ of these costs. The total costs of ABMT amounted to US $\$$ 40220.

\subsubsection{Treatment cost AML group}

In general, the AML patients received three induction therapies as first-line treatment and 1-3 reinduction therapies as second-line treatment. We calculated the average costs of these induction therapies (Table 11.2) and used this calculation as the basis for the reinduction treatment. 
Table 11.2. Average costs and days in hospital of induction treatments in AML

\begin{tabular}{|l|c|c|}
\hline & $\begin{array}{c}\text { Costs induction } \\
\text { treatment (in US\$) } \\
\text { (range) }\end{array}$ & $\begin{array}{c}\text { Average number } \\
\text { of hospital days } \\
\text { (range) }\end{array}$ \\
\hline Cycle 1: DNR and Ara-C & $30560(22640-42080)$ & $33(25-44)$ \\
Cycle 2: Ara-C and amsacrine & $35040(22640-50540)$ & $36(25-59)$ \\
Cycle 3: Mitoxantrone and Ara-C & $27880(14590-39130)$ & $36(23-53)$ \\
Average cost/days of 3 induction cycles & 93480 & 105 \\
Average cost/days per induction cycle & 31160 & 35 \\
\hline
\end{tabular}

All patients were hospitalised. The mean number of days in hospital amounted to 35 days (range: 23-59 days). The average costs of an induction cycle amounted to about US\$ 31160. When patients remained in complete remission, the costs of follow-up only were US\$ 4220. The average costs of follow-up in the "no further treatment" option amounted to approximately US\$11040 (Table 11.3).

With ABMT, in option 2 in addition to option 3, BM was harvested after the first $\mathrm{CR}$. The same procedure for the BM harvest was accounted for as for the NHL patients. In the Netherlands, BM of leukaemia patients is also usually unpurged. Therefore, the same costs (US\$ 5480) were used. In option 3, about 1.5 of the BM harvests actually resulted in an ABMT. Hence, with this treatment option the pre-transplantation costs amounted to about US $\$ 8220$.

Costs of the transplantation period were more expensive than those incurred with the lymphoma patients, because the AML patients stayed longer in the hospital and needed more antibiotics and blood transfusions. The costs of this period amounted to about US\$ 43000. Costs of hospitalisation were responsible for almost $55 \%$ of the total costs, medical procedures for $13 \%$, medication for $14 \%$ and blood transfusions for $18 \%$. Total ABMT costs were about US $\$ 51220$. 
Table 11.3 Costs of treatment for AML in the Netherlands

\begin{tabular}{|c|c|c|}
\hline & $\begin{array}{r}\text { Treatment } \\
\text { cost } \\
\text { (US\$) }\end{array}$ & $\begin{array}{r}\text { Average } \\
\text { cost } \\
\text { (US\$) }\end{array}$ \\
\hline $\begin{array}{l}\text { "No further treatment" (option } 1 \text { ) } \\
-40 \% \text { no relapse } \\
-60 \% \text { relapse: } \quad .50 \% \text { re-induction cycle } \\
\\
\text { Total option } 1\end{array}$ & $\begin{array}{r}4220 \\
31160 \\
0\end{array}$ & $\begin{array}{r}1690 \\
9350 \\
0 \\
11040\end{array}$ \\
\hline $\begin{array}{l}\text { "No further treatment" (option } 2 \text { ) } \\
- \text { Bone marrow harvest } \\
-40 \% \text { no relapse } \rightarrow \text { no further treatment } \rightarrow \text { follow up } \\
-60 \% \text { relapse: } \\
.90 \% \text { received } 1 \text { re-induction cycle: } \\
-50 \% \text { second } \mathrm{CR}: \\
\quad .85 \% \text { no further treatment } \\
.15 \% \text { ABMT } \\
-50 \% \text { no second } \mathrm{CR} \rightarrow \text { no further treatment } \\
.10 \% \text { no further treatment } \\
\text { Total option } 2\end{array}$ & $\begin{array}{r}5480 \\
4220 \\
31160 \\
0 \\
43000 \\
0 \\
0\end{array}$ & $\begin{array}{r}5480 \\
1690 \\
16830 \\
0 \\
1740 \\
0 \\
0 \\
25740\end{array}$ \\
\hline $\begin{array}{ll}\text { ABMT (option 3): } & \\
\text { ABMT } & \\
\text { Follow-up: } & -60 \% \text { no relapse } \\
& -40 \% \text { relapse } \\
\text { Total option } 3 & \end{array}$ & $\begin{array}{r}51220 \\
4220 \\
4220\end{array}$ & $\begin{array}{r}51220 \\
2530 \\
1690 \\
55440\end{array}$ \\
\hline
\end{tabular}

In option 2, the average costs of treatment were approximately US $\$ 25740$. However, as the purpose of this study was to assess the costs of introducing ABMT in the Netherlands and option 2 included an ABMT option, we calculated the costs of introduction as the difference between option 3 and option 1. In the ABMT group, about $40 \%$ of the patients would develop a relapse and received no further therapy. The costs in the follow-up period amounted to about US $\$ 4220$. 


\subsubsection{Costs of introducing ABMT}

In the Netherlands, the expected number of ABMTs was 180 for patients with NHL and the expected number for patients with AML was estimated at 50 (Medical Insurance Board, 1993). The costs of introducing ABMT in both patient groups are summarised in Table 11.4. When conventional NHL treatment with chemotherapy is replaced by ABMT, the minimum substitution costs amount to about US\$27430 and the maximum costs to about US\$37120. By multiplying these costs with the expected number of patients, costs of introducing ABMT for this patient group will vary between 4.93 and 6.68 million dollars.

Table 11.4. Costs of introducing ABMT in patients with NHL and AML in the Netherlands

\begin{tabular}{|l|r|r|r|}
\hline Costs per patient per group & $\begin{array}{r}\text { Number } \\
\text { of } \\
\text { patients }\end{array}$ & $\begin{array}{r}\text { Average } \\
\text { costs } \\
\text { (US\$) }\end{array}$ & $\begin{array}{r}\text { Total costs } \\
\text { (x 1000) } \\
\text { (US\$) }\end{array}$ \\
\hline NHL group: & & & \\
- ABMT & 180 & 40220 & 7240 \\
- Chemotherapy on inpatient basis & & 12900 & 2306 \\
- Chemotherapy on outpatient basis & & 3120 & 562 \\
Costs of ABMT introduction & & & \\
- Minimum & & 27410 & 4934 \\
- Maximum & & 37100 & 6678 \\
\hline AML group: & & & \\
- ABMT & 50 & 55440 & 2772 \\
- Conventional treatment & & 11040 & 552 \\
Costs of ABMT introduction & & 44400 & 2220 \\
\hline
\end{tabular}

In the AML group the extra costs of introducing ABMT are assessed at 2.22 million dollars, that is US $\$ 4400$ per patient. Combining both patient groups, the total costs of introducing ABMTs in the Netherlands are expected to be between 7.15 million and 8.90 million dollars. 


\subsection{Discussion}

As new treatment modalities become available for patients with haematological malignancies, the additional economic burden raises concern (Weber, 1993). This analysis shows that at least some savings from substitution will occur. However, there still remains a gap, and hospitals which carry out ABMTs will not be able to finance these additional costs from savings elsewhere.

In the Netherlands ABMT is also used for haematological malignancies, besides for NHL and AML, namely patients with Hodgkin's disease and acute lymphoid leukaemia (ALL). Patients with Hodgkin's disease are conditioned for transplantation if they do not attain a complete remission on first-line chemotherapy or present with relapse following $\mathrm{CR}$ within 1 year after chemotherapy. Patients with ALL are transplanted if they relapse and reach a second remission. According to the panel of haematological experts, the use of medical resources by patients with Hodgkin's disease is almost comparable to the NHL patients and the use of medical facilities of ALL patients is comparable to that of patients with AML. The total number of ABMTs per year is estimated at 50 for Hodgkin's disease and 25 for ALL (Medical Insurance Board, 1993). As a result, the total extra costs of introducing ABMT for the lymphoma and acute leukaemia patients, including these last two categories, are expected to be between 9.63 and 11.86 million dollars.

In our study, the estimated cost of ABMT was lower than reported in other studies. In the literature, the estimated cost of ABMT for lymphoma and breast cancer varied between US\$ 62200 and US\$100000 (Desch et al., 1992; Hillner et al., 1992; Gulati and Bennet, 1992). The cost of salvage therapy was estimated at $\$ 4000$ and $\$ 15000$. With leukaemia patients, Welch and Larson (1989) estimated the ABMT costs at $\$ 139000$ and the chemotherapy costs at $\$ 78000$, both during the first 6 months. This high ABMT cost, compared with our results, may be related to the fact that in these studies all patients who underwent transplantation, regardless of their medical condition were treated in the intensive care unit or in a protected care unit during the entire transplantation period. In the study of Dufoir et al. (1992), patients were hospitalised in a protected care unit from the date of admission to the end of aplasia. Therefore, the estimated costs of ABMT were lower, namely about US $\$ 72400(1 \mathrm{FF} \approx 0.184$ US $\$$ ). The costs of ABMT were still higher than by our calculations, but in this study a number of patients received a double transplantation. Conventional chemotherapy (four cycles) amounted to US $\$ 24000$; the mean number of days in hospital was 23 days, that is $2-5$ days per cycle. The cost difference in the various studies are mostly generated by the difference in the ABMT procedures. Therefore, it may be advisable to specify the ABMT procedure and to present 
procedure-specific unit prices in more detail, allowing better comparison of ABMT costs between countries.

The introduction of ABMT has major economic consequences, but it has important treatment benefits as well. For the NHL patients mentioned above, the disease-free survival (DFS) at 2 years after DHAP varies between $5 \%$ and $20 \%$ (Velasquez et al., 1988). ABMT may result in a DFS at 3 years of approximately $50 \%$ of all transplanted patients and in $25 \%$ of the original number of patients destined to receive ABMT (Verdonck et al., 1992). Patients with Hodgkin's disease after a chemotherapy relapse were treated with similar, or salvage chemotherapy. Although $35-60 \%$ of these patients achieve a $\mathrm{CR}$, only $25-35 \%$ are alive at 5 years. ABMT after first relapse frequently results in higher CR rates and longer DFS (Desch et al., 1992). According to the panel, with no $A B M T$, patients with AML in first remission have a $40 \%$ probability remaining in remission. With ABMT this probability is about $60 \%$. These estimates correspond with the results of Welch and Larson (1989). Furthermore, Welch and Larson estimated the 5years survival at $41 \%$ for the conventional chemotherapy group and at $60 \%$ for the ABMT group. For ALL patients who relapse, second CRs can be obtained in $10-75 \%$ of patients depending on the treatment regimens; however the durability of these remissions is poor. ABMT in these patients does lead to significant longer DFS (Ball and Rybka, 1993).

Currently, additional treatment modalities in these patient groups are being studied (Hillner et al., 1992; Velasquez et al., 1988; Uyl-de Groot et al., 1994II). Use of colony stimulating factors and peripheral stem cell transplantation (PSCT) seem to be alternatives to ABMT. Fortunately, it may be expected that these modalities are cheaper than ABMT (Nemunaitis et al., 1991). However, in the near future, it is also expected that ABMTs or stem cell transplants will be carried out in patient groups not previously treated with transplantation (Hillner et al., 1992; Ayash et al., 1994; Ossenkoppele et al., 1994). Economic evaluations linked to clinical trials provide information about the cost-effectiveness of new treatment modalities. In the Netherlands several economic evaluations concerning PSCT are being carried out, for example in breast carcinoma and multiple myeloma. When these new treatment modalities are implemented in the clinical setting, these studies should provide the necessary information on the economic consequences of introducing such new technologies. 


\section{A glossary of chemotherapy regimens}

CHOP: $\quad$ Cyclophosphamide, doxorubicin, vincristine, prednisone

ProMACE-MOPP: Prednisone, methotrexate with leucovorin rescue doxonibicin, etoposide, mechlorethamine, vincristine, prednisone, procarbazine

CAMP: Cyclophosphamide, adriamycin, mitoxantrone, prednisone

DHAP: Dexamethason, cisplatine, Ara- $\mathrm{C}$, prednisone

IMVP: Ifosfamide, methotrexate, etoposide (VP-16) 


\section{Hospital economic model for HGF therapy}

\subsection{Summary}

Patients receiving chemotherapy frequently develop fever and neutropenia. Haematopoietic growth factors (HGFs) may decrease the duration of such episode or may prevent a febrile neutropenic episode. In this study we introduce a Markov type economic model for the hospital which calculates all relevant direct costs and savings of HGF therapy and may support decisions on HGF administration. A distinction is made between patients receiving intensive and standard chemotherapy schedules. Our results indicate that HGFs can induce savings in intensive chemotherapy and standard chemotherapy following neutropenic fever. Prophylactic administration of $\mathrm{HGF}$ is cost-effective if the risk of infection is considerable. The risk of infection depends on underlying malignancy, corresponding treatment modalities and the health condition of the patient. The model is meant as an analytical framework and should be used carefully, as not all benefits (e.g. benefits to the patients) are considered. These benefits may be balanced against the additional costs or savings resulting from the economic model.

\subsection{Introduction}

Neutropenia is closely related with the treatment of cancer. The risk of a life-threatening infection increases significantly when the neutrophil count falls below $0.5 \times 10^{9} / 1$ and it is a major cause of death in cancer patients. Furthermore, the duration of neutropenia has an important role in the design, schedule, and dose of cancer treatment regimens (Antman et al., 1988; Pizzo, 1984). Treatment-related neutropenia is highly variable, ranging from days to weeks. 
Neutropenic patients with fever require prompt initiation of early broad spectrum antibiotic therapy. Moreover, haematopoietic growth factors (HGFs), such as granulocyte colony-stimulating factor (G-CSF) and granulocyte-macrophage colony-stimulating factor (GM-CSF) are becoming increasingly important in the treatment of febrile neutropenic patients (Liesclike and Burgess, 1992; Metcalf, 1990). HGFs are indicated for rapid haematopoietic reconstitution by stimulating the proliferation of neutrophils and could be administered during intensive chemotherapy schedules, during standard chemotherapy cycles and in patients with chemotherapy-induced fever and neutropenia (Biesma et al., 1990; Crawford et al., 1991; Bronchud et al., 1987; Estey et al., 1992).

The administration of HGFs during intensive chemotherapy regimens mainly concerns patients undergoing autologous bone marrow transplantation (ABMT) or peripheral blood progenitor cell (PBPC) transplantation and patients with acute myeloid leukaemia (AML). These patients remain neutropenic for three to four weeks (Estey et al., 1992; Ohno et al., 1990; Uyl-de Groot et al., 1994II). They are all hospitalised and treated with broad spectrum antibiotics. HGF therapy may reduce the period of neutropenia and may result in shortening the period of hospitalisation and antibiotic therapy.

Prophylactic administration of HGF during and after standard chemotherapy cycles is already applied in patients with small cell lung cancer (Crawford et al., 1991; Bronchud et al., 1987; Trillet-Lenoir et al., 1993). The standard chemotherapy is mainly given on an outpatient basis. When patients become febrile and neutropenic during a given cycle of treatment, they are generally hospitalised and receive standard parenteral antibiotic agents (Metcalf, 1990; Estey et al., 1992; Riikonen et al., 1994; Gabrilove, 1988).

At a time of restrained public spending, hospitals are coming under increasing pressure to deliver more services with less resources. The main cause of rising hospital drug expenditure in leading Western European countries are antibiotics (Thürmann and Harder, 1993). The administration of HGFs may lead to less infections and as a result to less antibiotic administrations. Furthermore, it could result in a lower risk of hospitalisation and a shorter stay in hospital. Thus, the use of HGFs may decrease utilisation of some health care resources. Glaspy et al. (1993) developed an economic model to demonstrate these savings of prophylactic administration of the haematopoietic growth factor G-CSF (filgrastim) in patients with small cell lung cancer. The analysis was conducted in conjunction with a phase III clinical trial (Crawford et al., 1991). The data were derived from 3 of the 14 hospitals included in the clinical trial. However, the clinical results in these 3 hospitals differed from the results of the total study. For example, in the larger trial the number of days in hospital and the number of days with antibiotic therapy were 
similar in both groups once patients were hospitalised, while in the economic model not only the number of hospitalisations decreased with almost $50 \%$, but the duration of hospital stay decreased with almost $50 \%$ as well. Furthermore, Glaspy's study is focused on the administration of haematopoietic growth factors in one patient group.

The aim of this study is to develop an economic model for the hospital, which includes all relevant direct costs and savings in relation to chemotherapy-induced fever and neutropenia. Three categories of treatment choices will be presented: the first category considers a treatment option where HGFs are administered during intensive chemotherapy cycles. The second category describes a prophylactic option where HGFs are administered during and after standard chemotherapy cycles, which are given on an outpatient basis. When the patients become febrile and neutropenic, they are hospitalised and treated with broad spectrum antibiotics. The aim of HGF administration in this second category is to prevent patients from developing fever and neutropenia. It may be applied during the first chemotherapy cycle or during consequent cycles. The third category concerns a treatment option in for example solid tumour patients and patients with non-Hodgkin's lymphoma. These patients receive standard chemotherapy cycles again on an outpatient basis and subsequently may develop fever and neutropenia. The purpose of administering antibiotics in combination with HGFs is to shorten the period of fever and neutropenia.

The model is meant for hospital pharmacists, doctors and hospital management. The perspective of the study is that of the hospital and therefore only the direct costs of outpatient and inpatient treatment are considered.

\subsection{Approach and method}

\subsubsection{Development of the model}

The costs of the treatment alternatives will be compared by subtracting the estimated total costs used for the group treated with conventional therapy from the estimated total costs for the group treated with HGF therapy. For all categories, the following formula is used to calculate the cost or benefit of the HGFs therapy:

$\Delta \mathrm{C}=\Sigma \mathrm{C}_{1}-\Sigma \mathrm{C}_{2}$

where $\Delta \mathrm{C}=$ differential treatment cost, $\Sigma \mathrm{C}_{1}=$ total treatment costs of convential therapy without $\mathrm{HGF}$ and $\Sigma \mathrm{C}_{2}=$ total treatment costs of HGF therapy. 
This formula implies that if $\Delta \mathrm{C}$ is positive, the conventional therapy is more expensive than HGF therapy. The situation with $\Delta \mathrm{C}=0$ marks the break-even point, and if $\Delta \mathrm{C}$ $<0$ than the administration of HGFs does not lead to savings.

Figure 12.1 shows the three categories in the hospital economic model. In category $I$, all patients are treated with intensive chemotherapy and stay in hospital for a few weeks. During and after the administration of cytostatics they are treated either with antibiotics or with antibiotics in combination with HGF therapy. The relevant cost items are days in hospital, days with antibiotics and, if applicable, the number of days with HGF. This results in the following formula:

$$
\begin{aligned}
\Delta \mathrm{C}_{\mathrm{l}}= & \left\{\left(\mathrm{HD}_{11} \times \mathrm{HC}_{1}\right)+\left(\mathrm{AD}_{11} \times \mathrm{AC}_{\mathrm{l}}\right)\right\}- \\
& \left\{\left(\mathrm{HD}_{12} \times \mathrm{HC}_{1}\right)+\left(\mathrm{AD}_{12} \times \mathrm{AC}_{\mathrm{I}}\right)+\left(\mathrm{HGFD}_{1} \times \mathrm{HGFC}\right)\right\} .
\end{aligned}
$$

where $\Delta \mathrm{C}_{\mathrm{I}}=$ differential costs in category $\mathrm{I}, \mathrm{HD}_{\mathrm{l}}=$ number of hospital days, $\mathrm{HC}_{\mathrm{I}}=$ cost per hospital day, $\mathrm{AD}_{1}=$ number of days with antibiotic therapy, $\mathrm{AC}=$ antibiotic cost per day, $\mathrm{HGFD}_{1}=$ number of days with $\mathrm{HGF}$ therapy and $\mathrm{HGFC}=\cos$ of $\mathrm{HGF}$ per day.

Category II concerns patients who receive standard chemotherapy. These patients may be treated with HGF on a prophylactic basis. This means that all patients receive HGF directly after completing their chemotherapy. When the HGF is not applied, the so called "wait and see" option, there is a probability that fever and neutropenia develop (PFN1). Once this event has set in, antibiotics or antibiotics plus HGFs are administered (category III). Delay of administration of the following chemotherapy cycle may occur, resulting in extra outpatient visits. The relevant cost items are days in hospital, days with antibiotics and HGF, and number of extra outpatient visits. For this category the following formula applies:

$$
\begin{aligned}
& \Delta \mathrm{C}_{\mathrm{Il}}=\left\{\mathrm{P}(\mathrm{FN} 1) \times\left(\left(\mathrm{HD}_{\mathrm{II1}} \times \mathrm{HC}_{\mathrm{ll}}\right)+\left(\mathrm{AD}_{\mathrm{II1}} \times \mathrm{AC}_{\mathrm{II}}\right)+\left(\mathrm{P}\left(\mathrm{EO}_{\mathrm{II1}}\right) \times \mathrm{EO}_{\mathrm{II1}} \times \mathrm{OC}_{\mathrm{Il}}\right)\right)\right\}- \\
& \left\{\left(\mathrm{P}(\mathrm{FN} 2) \times\left(\left(\mathrm{HD}_{112} \times \mathrm{HC}_{\mathrm{II}}\right)+\left(\mathrm{AD}_{\mathrm{II2}} \times \mathrm{AC}_{\mathrm{II}}\right)+\left(\mathrm{P}\left(\mathrm{EO}_{\mathrm{II}}\right) \times \mathrm{EO}_{\mathrm{HI}} \times \mathrm{OC}_{\mathrm{II}}\right)\right)\right)+\right. \\
& \left.\left(\mathrm{HGFD}_{11} \times \mathrm{HGFC}\right)\right\}
\end{aligned}
$$

where $\Delta C_{I I}=$ differential costs in category II, $\mathrm{P}(\mathrm{FN})=$ probability to develop fever and neutropenia, $\mathrm{HD}_{\mathrm{II}}=$ number of hospital days, $\mathrm{HC}_{\mathrm{II}}=$ cost per hospital day, $\mathrm{AD}_{\mathrm{II}}=$ number of days with antibiotics, $\mathrm{AC}_{\mathrm{II}}=$ antibiotic cost per day, $\mathrm{P}\left(\mathrm{EO}_{\mathrm{II}}\right)=$ probability of extra outpatient visits, $\mathrm{EO}_{\mathrm{II}}=$ number of extra outpatient visits, $\mathrm{OC}_{\mathrm{II}}=\cos$ of an outpatient visit, $\mathrm{HGFD}_{\mathrm{Il}}=$ number of days with $\mathrm{HGF}$, and $\mathrm{HGFC}=\mathrm{HGF}$ costs per day. 
Figure 12.1 Hospital economic model

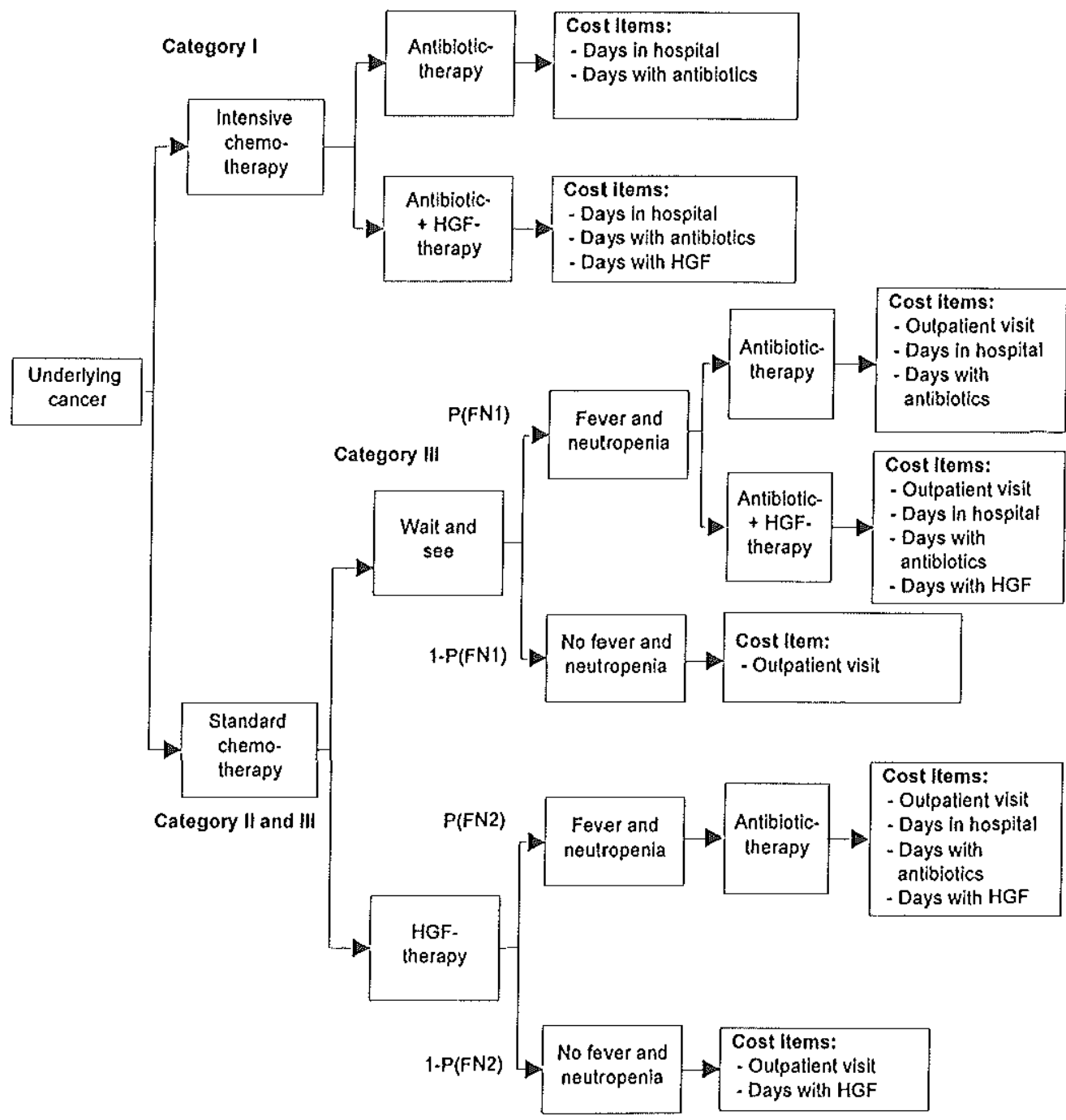

Category III handles patients who develop fever and neutropenia due to standard chemotherapy. The chemotherapy is administered on an outpatient basis, but the patients are hospitalised in case of fever and neutropenia. Delay of chemotherapy administration may happen in both groups. The formula is the same formula as for category 1 . 
Default values of the probability to develop fever and neutropenia, the hospitalisation risk, the number of days in hospital, the number of extra outpatient visits, the number of days with antibiotics and the number of days with a HGFs are based on literature review. Cost prices are derived from previous Dutch studies on the cost-effectiveness of cancer treatment in the relevant patient groups (Uyl-de Groot et al,, 1994I and 1994II). Sensitivity analyses are carried out to provide insight into the effect of changes in the probability to develop fever and neutropenia, days in hospital and cost per hospital day on the total treatment costs.

We specified a Markov model to estimate the consequences of HGF administration when more (standard) chemotherapy cycles are given and possible combinations of category II and III are considered. Patients treated with antibiotics or HGF therapy in the previous cycle are assumed to receive the same treatment in the following cycles.

We used the computer programme Quattro Pro for Windows.

\subsubsection{Default values}

Category I: In the study of Nemunaitis et al. (1991), the median duration of the initial hospital stay was 27 days in the HGF group as compared to 33 days in the placebo group. This is a reduction of almost $20 \%$ and is consistent with results reported elsewhere (Gorin et al., 1992). The number of days with antibiotics was about $82 \%$ of the days in hospital in the placebo group. This percentage was $89 \%$ in the HGF group. For the formula, this implies that: $\mathrm{HD}_{12}=0.8 \times \mathrm{HD}_{11}, \mathrm{AD}_{11}=0.82 \times \mathrm{HD}_{11}$ and $\mathrm{AD}_{12}=0.89 \times \mathrm{HD}_{12}$.

In category II, the conventional therapy is the "wait and see" option. Patients only receive antibiotics when they develop fever and neutropenia. The default values will be similar as in category III. The other option is that all patients receive HGF therapy. HGFs are given on average 10 days (range 7-14 days) (Crawford et al., 1991; Bronchud et al., 1987; Gerhartz et al., 1993). Crawford et al. (1991) and Bronchud et al. (1987) reported a reduction of the event rate for fever and neutropenia by $50 \%$ in the HGF group as compared with the "wait and see" option. The average duration of the individual episodes of antibiotic use and hospital stay in case of fever and neutropenia, were similar in both treatment groups. In the study of Gerhartz et al. (1993) the event rate decreased with $40 \%$. The reduction in infection distributed equally through all six cycles of chemotherapy. Patients receiving HGF stayed shorter in hospital than the patients treated with placebo. The percentage of chemotherapy cycles finished on time was $54 \%$ in the HGF 
group and $34 \%$ in the placebo group. However, data on the average number of delayed chemotherapy schedules per patient were not showed. To simplify the models these probabilities are set to zero. The assumptions made are $\mathrm{P}(\mathrm{FN} 2)=0.5 \times \mathrm{P}(\mathrm{FN} 1), \mathrm{HGFD}_{\mathrm{II}}$ $=10$ and once the event has set in: $\mathrm{HD}_{\mathrm{II1}}=\mathrm{HD}_{\mathrm{II} 2}, \mathrm{AD}_{\mathrm{II1}}=\mathrm{AD}_{\mathrm{II} 2}$ and $\mathrm{P}\left(\mathrm{EO}_{\mathrm{II1}}\right)=$ $\mathrm{P}\left(\mathrm{EO}_{\mathrm{II} 2}\right)=0$.

Category III: In the study of Mayordomo et al. (1993), patients treated with antibiotic therapy had a median hospital stay of 8 days (range: 5-34) and patients treated with antibiotic therapy in combination with HGF had a median hospital stay of 5 days (range: 515). This implies a reduction of $37 \%$ in hospital days. In the study of Maher et al. (1994), the median number of days in hospital amounted to 8 days in both groups, while the mean number of days in hospital amounted to 10.0 days in the antibiotic group and to 8.7 days in the HGF group. The difference in hospital stay between the studies of Mayordomo and Maher could be explained by differences in definition of fever and neutropenia and differences in doses of HGF administration. In the model we assume that the hospital stay in the antibiotic group is 10 days and 8 days in the antibiotics with HGF group, implying a reduction with $20 \%$. The antibiotic therapies consisted of piperacillin and tobramcyin, ceftazidim and amkacin or tobramycin and cefuroxime (Biesma et al., 1990; Gerhartz et al., 1993; Mayordomo et al. 1993). The antibiotic therapy and/or HGF therapy was stopped about 2 days before hospital discharge. This implies that: $\mathrm{HD}_{\mathrm{III} 2}=$ $0.8 \times \mathrm{HD}_{\mathrm{III}}, \mathrm{AD}_{\mathrm{III}}=\left(\mathrm{HD}_{\mathrm{IIII}}-2\right)$ and $\mathrm{AD}_{\mathrm{III}}=\mathrm{HGFD} \mathrm{HII}=\left(\mathrm{HD}_{\mathrm{III}}-2\right)$.

For the Markov model all default values will apply. Data on patients developing FN in subsequent cycles are not available. According to interviews with clinical experts, we made the assumption that patients with a previous FN have a $95 \%$ chance of FN in the next cycle and that patients with no previous FN have a $5 \%$ chance of FN in the next cycle.

\subsubsection{Unit prices}

In the Netherlands, the cost of hospitalisation amounts to about US\$350 per day for normal haematological care, to about US $\$ 536$ per day for a stay in a protected environment and to about US $\$ 1223$ per day for a stay on an intensive care ward (Uyl-de Groot et al., 1994I and 1994II). These costs do not include the costs for laboratory services and medical procedures. The laboratory services mainly consist of routine haematological laboratory services and cultures. The medical procedures include X-rays and ECGs. We estimate these costs at US\$50 per hospital day. 
The patients who receive intensive chemotherapy stay more frequently and longer in a protected environment and/or on an intensive care ward. Furthermore, in the Netherlands these patients are treated in haemato-intensive care hospitals, thus the hospital costs are in this case higher than in the standard chemotherapy strategies. We use US $\$ 600$ as an estimation for the average cost of a day in hospital for the patients receiving intensive chemotherapy schedules. The costs for laboratory services and medical procedures are included in this price. For the patients who receive standard chemotherapy, we estimated a cost price of US $\$ 450$ for a hospital day in a regional hospital. The costs of antibiotic therapy are estimated at US $\$ 100$ per day. The recommended amount of HGF varied from $3.5 \mu \mathrm{g} / \mathrm{kg}$ to $12 \mu \mathrm{g} / \mathrm{kg}$ per day. In the model, we use a unit price of US\$ 138 , that is the cost of a $300 \mu \mathrm{g}$ vial G-CSF or GM-CSF.

\subsection{Results}

\section{Category I: Group receiving intensive chemotherapy.}

The results of the costs of convential antibiotic treatment and antibiotic plus HGF therapy are summarised in Table 12.1. The antibiotic therapy costs are about US $\$ 22510$ and the antibiotic plus HGF therapy cost about US $\$ 21550$. The model suggests that antibiotic plus HGF therapy results in a saving of US\$ 960 in comparison with a therapy with antibiotics alone.

In Figure 12.2 the number of days in hospital when no HGF is given $\left(=\mathrm{HD}_{\mathrm{I1}}\right)$ is varied from 25 to 40 days. The model shows that the level of savings varies from US $\$ 28$ (HD $=25$ days) to US $\$ 1780\left(\mathrm{HD}_{11}=40\right.$ days) (Figure 12.2). For this range the conventional therapy is more expensive than the antibiotics plus HGF therapy.

Sensitivity analyses were performed to assess the effect of changes in the number of days in hospital and the cost per hospital day. The cost per hospital day is varied from US $\$$ 400 to US $\$ 800$. When the hospital cost is lower than the default value of US $\$ 600$, the savings decrease obviously. For example, when the hospital cost is US $\$ 400$, the savings range from US $\$-870\left(\mathrm{HD}_{11}=25\right.$ days) to US $\$ 340$ ( $\mathrm{HD}_{11}=40$ days). The break-even point, i.e. no cost difference between the two treatment groups, is at 35.8 days.

When the hospital costs are higher than the default value, for example US $\$ 800$, the savings are higher and range from US $\$ 930\left(\mathrm{HD}_{11}=25\right.$ days) to US $\$ 3220\left(\mathrm{HD}_{11}=40\right.$ days). When the number of days in hospitals decreases with a smaller percentage due to HGF therapy than the default value of $20 \%$ reduction, the savings are smaller. 
Table 12.1 Costs of conventional and HGF therapy per category (in US\$)

\begin{tabular}{|c|c|c|c|}
\hline Categories & $\begin{array}{r}\text { Costs of } \\
\text { antibiotic } \\
\text { therapy }\end{array}$ & $\begin{array}{l}\text { Costs of } \\
\text { antibiotic- } \\
+ \text { HGF- } \\
\text { therapy }\end{array}$ & $\begin{array}{r}\text { Cost } \\
\text { difference }\end{array}$ \\
\hline \multicolumn{4}{|l|}{ Category I (intensive chemotherapy group) } \\
\hline - Hospital costs & 19800 & 16240 & 3560 \\
\hline - Costs of antibiotics & 2710 & 2410 & 300 \\
\hline - Costs of HGFs & - & 2900 & -2900 \\
\hline Total costs & 22510 & 21550 & 960 \\
\hline \multicolumn{4}{|l|}{ Category II (standard chemotherapy group) } \\
\hline - Hospital costs & 2565 & 1280 & 1285 \\
\hline - Costs of antibiotics & 455 & 230 & 225 \\
\hline - Costs of HGFs & - & 1380 & -1380 \\
\hline Total costs & 3020 & 2890 & 130 \\
\hline \multicolumn{4}{|l|}{ Category III (standard chemotherapy group) } \\
\hline - Hospital costs & 4500 & 3600 & 900 \\
\hline - Costs of antibiotics & 800 & 600 & 200 \\
\hline - Costs of HGFs & 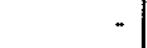 & 828 & -828 \\
\hline Total costs & 5300 & 5028 & 272 \\
\hline
\end{tabular}

Assumptions: $\mathrm{P}(\mathrm{FN} 1)=57 \% ; \mathrm{HD}_{\mathrm{II}, \mathrm{tm}}=10$; default values.

Category II: Group receiving standard chemotherapy \pm prophylactic HGF therapy.

Now the cost of fever and neutropenia treatment with and without a HGF depend on the probability to develop fever and neutropenia $(=\mathrm{P}(\mathrm{FN}))$. The chance of savings increases with the risk of fever and neutropenia. A relatively small risk of fever and neutropenia results in ineffective HGF administration in many patients and leads to additional net costs. Taking into account the default values and a $\mathrm{P}(\mathrm{FN} 1$ ) of $57 \%$ (Crawford $e t$ al., 1991) there will be a saving of the HGF therapy of US $\$ 130$ (Table 12.1).

A sensitivity analysis was performed to assess the impact of varying the risk of developing fever and neutropenia from $0 \%$ to $100 \%$ (Figure 12.3). When the hospital cost is US $\$$ 450 , the savings varied from US $\$-1380$ to US $\$ 1470$. The break-even point is at $52 \%$, implying that below this level the HGF strategy is more expensive than the conventional antibiotic treatment. 
Figure 12,2 Group receiving intensive chemotherapy (calegory 1 )

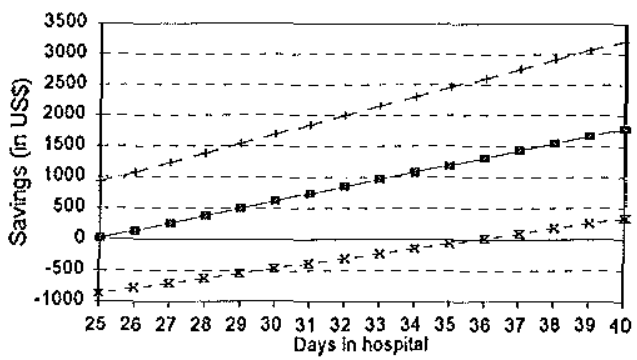

$-H C=U S \$ 600-x \cdot H C=U S \$ 400+H C=$ US $\$ 800$

Figure 12.4 Group receiving standard chemotherapy (category III)

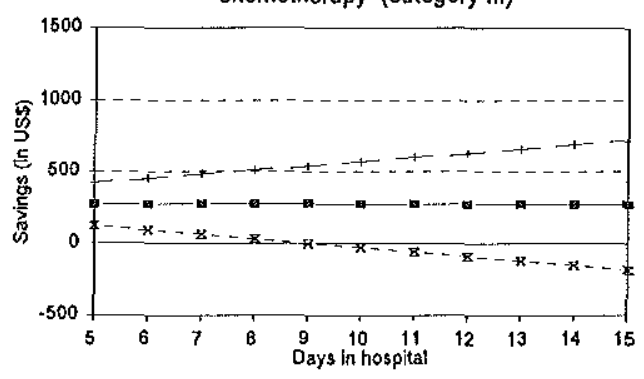

$\cdot H C=U S \$ 450 \cdot r \cdot H C=U S \$ 300+H C=$ US\$ 600
Figure 12.3 Group receiving standard chemotherapy (category II)

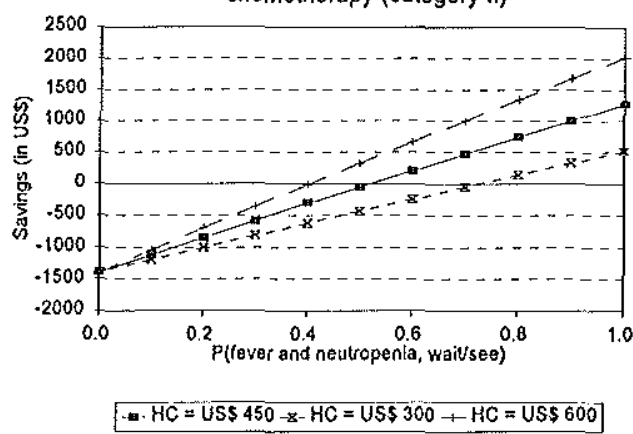

Figure 12.5 Groups receiving 3 cycles

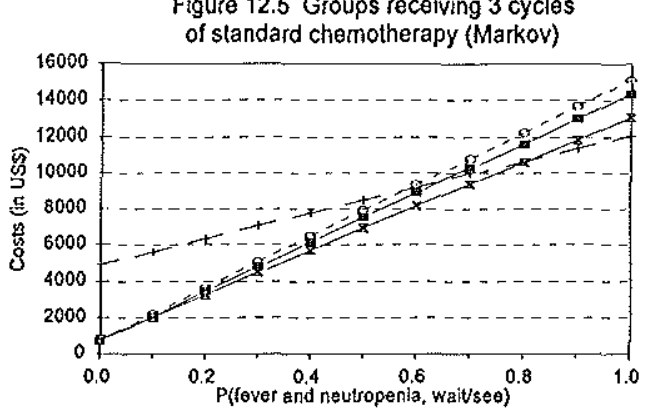

-o- Conventional antiblotic therepy $\rightarrow$ KGF + antiblotic therapy + Prophylactic HGF therapy $\rightarrow$ - Prophylactic HGF after FN

Furthermore, we varied the price of a hospital day with US $\$ 150$. The a priori chance of $\mathrm{P}(\mathrm{FN} 2)$ still is $0.5 \times \mathrm{P}(\mathrm{FN} 1)$. When the hospital cost is as low as US\$300, the savings vary from US\$ -1380 to US\$ 520 . The break-even point is at about $72.7 \%$. In case the price of a bed day is US\$ 600 , the savings range from US\$ -1380 to US $\$ 2220$ and the break-even point is at $38.4 \%$.

When $\mathrm{P}(\mathrm{FN} 2)$ decreases to less than $50 \%$ of $\mathrm{P}(\mathrm{FN} 1)$, the savings are smaller. A more than $50 \%$ reduction of $\mathrm{P}(\mathrm{FN} 1)$ results in more savings.

Category III: Group receiving standard chemotherapy, developing fever and neutropenia. In this group the average duration of the hospitalisation period is shorter than in the group receiving intensive chemotherapy. Furthermore, the cost per day in hospital will be 
lower. Taking into account the default values, the costs in the antibiotic group amount to US $\$ 5300$ and in the HGF group US $\$ 5028$ (Table 12.1). This results in a saving of US\$ 272 in favour of the HGF therapy. Figure 12.4 shows that in this case the outcomes are not very sensitive for changing the number of days in hospital. The savings range from US\$ 274 to US\$270.

When the hospital cost amounts to US $\$ 300$, the savings range from US $\$ 124\left(\mathrm{HD}_{1}=5\right.$ days) to US $\$-180\left(\mathrm{HD}_{1}=15\right.$ days). The break-even point is at 9.0 days. Hospital cost of US\$600 per day always result in savings (range: US\$ 424 - US\$720).

\section{Markov model.}

Considering category III over 3 chemotherapy cycles and the default values, the cumulative costs amount to US\$ 8955 in the conventional antibiotic therapy group. When HGF is used as treatment option in case of fever and neutropenia, the cost amounts to US $\$ 8496$. Prophylactic use of HGF over 3 cycles results in cost of US $\$ 9002$. This implies that HGF as a treatment option in fever and neutropenic patients produces the lowest costs. However, this option is accompanied by having fever and neutropenia and its associated risks of (fatal) complications. Furthermore, it could result in dose reduction and delay of chemotherapy administration. The latter frequently requires extra outpatient visits. In the Netherlands, an outpatient visit costs approximately US\$90 (Uyl-de Groot et al., 1994II). Assuming that one delay of chemotherapy administration requires one extra outpatient visit, it already implies that a $50 \%$ delay of chemotherapy administration results in equal costs in the prophylactic strategy and the "wait and see" strategy with conventional antibiotic treatment.

Another option is to give HGF only prophylactic when patients had previous fever and neutropenia. The patients received HGF plus antibiotic therapy for their first febrile neutropenic episode. We assumed that probability of fever and neutropenia in the next cycle will be reduced with $50 \%$. Fever and neutropenia after prophylactic HGF therapy will be treated with antibiotics. Taking into account the default values and a $\mathrm{P}(\mathrm{FN} 1)$ of $57 \%$ the cumulative costs of this option amount to US\$7975.

A sensitivity analysis is performed to demonstrate the effect of changing the probability of developing fever and neutropenia. Figure 12.5 shows that when the probability of fever and neutropenia is lower than $80 \%$, prophylactic administration of HGF when patients had previous fever and neutropenia produces the lowest cost. Probabilities above $80 \%$ are in favour of the prophylactic HGF therapy during all cycles. 


\subsection{Discussion}

The administration of HGF is adding considerably to the cost of medication (Finley, 1991), but this hospital economic model indicates that the administration of HGF may lead to savings in the daily hospital practice. In the intensive chemotherapy and the "wait and see" option, HGF is cost-effective for almost all basic assumptions. However, standard chemotherapy is often administered in regional hospitals, where the cost of a hospital day is lower than in the haemato-intensive care hospitals. When patients stay longer than 9.0 days in hospital and/or the hospital cost are below US\$300, the HGF administration does not result in savings.

Concerning the prophylactic administration of HGF, whether or not there are savings, largely depends on the probability of developing fever and neutropenia. This probability differs between underlying malignancies, corresponding treatment modalities and the health condition of the patients. In our model, the administration of HGFs in patients with a high risk of infections leads to savings. This suggests that it is worthwhile to identify other determinants of high risks of infections, such as the prior patient history (infection at previous cycles) or other patient or hospital specific factors thought to influence the risk of infection.

This hospital economic model is meant as an analytical framework to assess the savings from a clinical application of HGFs. It is applicable to any hospital in any country, regardless of practice patterns as hospital and/or country specific data can be used. It may support decision making about the treatment of certain patient groups as well as negotiating budget transfers with hospital management. It only requires a few relatively simple data from the hospital accounting system and the medical registration system. One of the available spreadsheet programmes may be used to quickly calculate the differential costs given hospital and patient characteristic data.

However, the model should be used carefully, as the benefits to the patients, such as improved quality of life as a result of shorter stay in hospital and less infections are not considered. Other benefits may be less delay of chemotherapy administration, less distress to the patient and less travel costs. There may also be indirect savings (decreased loss of productivity). These benefits may be balanced against the additional costs (or added to the savings) resulting from the economic model. 


\section{Discussion}

\subsection{General issues}

Economic evaluation is intended to support health policy at different levels of decision making. In cancer treatment, health policy concerns both preventive, curative and palliative strategies and decisions are often made at the micro and macro level. Economic evaluation can provide essential information on the costs and benefits of each option and consequently on the optimal policy mix, and thus support decisions on the adoption and utilisation of new treatment strategies. Such information may assist policy makers in formulating regulatory policies and legislation, industry in developing products, health professionals in treating and serving patients, and consumers in making personal health decisions. Examples of situations in which economic evaluation can play a valuable role are:

Research and development decisions: The results of economic evaluation may be used for "go/no go" decisions at critical points in the development process of new treatment modalities. Information can be used both by university hospitals and pharmaceutical firms.

Pricing decisions: Economic evaluation can help to establish an appropriate price for a treatment modality. This information can be used both by government regulators and pharmaceutical firms.

Reimbursement decisions: Government, hospitals, insurers, and other payers can use the results of economic evaluation in their reimbursement policies. For instance, the results of economic evaluation could influence decision making on whether or not to include new technologies in the package of (social) health insurance. 
Clinical guidelines: Hospital budgets are limited and doctors face financial restrictions. The results of economic evaluation may support the choice of treatment strategy and the selection of patients at the micro level, as well as negotiating budget transfers with hospital management. While developing clinical guidelines for providers not only the efficacy and effectiveness of treatment alternatives need to be considered, but also their cost-effectiveness.

However, decisions should not be based on the results of economic evaluation alone. Economic evaluations are used to inform decision making, not to replace it. Using results of economic evaluations requires that these are reliable and unambiguous and that the evaluation is carried out in a rigorous manner. The methods used and the way in which costs and consequences are measured and valued must be made clear, in order to help policy makers to determine whether the study's results are relevant to their policy context. The following sections deal with specific issues concerning measuring and valuing outcome and resources and the generalisability of study results.

\subsection{Measuring and valuing outcome}

In clinical studies in cancer the main end points used are survival rates, response rates and response duration. To improve results in terms of incremental survival and less morbidity and suffering, physicians and nurses are now paying more attention to supportive care. Quality of life studies may give insight into the burden of cancer and its treatment for patients. Thus, quality of life is becoming an important end point in cancer research.

To incorporate quality of life aspects in economic appraisal, the use of a generic, a disease-specific, and a valuation instrument is advisable. In this respect standardisation is essential, so that different health care interventions can be compared. At the moment, in the Netherlands there is growing consensus among researchers which instruments should be used. In this thesis, the quality of life studies used the Karnofsky Performance Index and the Nottingham Health Profile as generic instruments, the Rotterdam Symptom Checklist as cancer-specific instrument and the EuroQoL as valuation instrument. The choice of instruments was based on the relatively good psychometric features, on their opportunity to capture specific patient features and their good feasibility record. 
Concerning the generic instruments, there is a tendency to use the Short-Form 36 instead of the Nottingham Health Profile. The Short Form-36 may be regarded as an extension of the RAND-MOS 20 (Ware et al., 1993); Anderson et al., 1993). It includes multi-item scales measuring eight health concepts (physical functioning, role limitations due to physical health problems, bodily pain, general health, vitality (energy/fatigue), social functioning, role limitations due to emotional problems, and mental health (psychological distress and psychological well-being), and a single item on change in health. The Short Form-36 has good psychometric features and the administration time is approximately 10 minutes (Ware $e t$ al., 1993). It is the only generic quality of life instrument reviewed that is currently undergoing international adaptation (Anderson et al., 1993). In the past, the use of Short-Form-36 was restricted, but recently it has become available for broader use. However, written approval of the Medical Outcome Trust is still required. In new economic appraisals concerning cancer treatments, for example in patients with renal cancer, in patients with multiple myeloma and in lymphoma patients undergoing stem cell transplantation, we used the Short-Form 36 in stead of the Nottingham Health Profile. A disadvantage of the Short-Form 36 is that this instrument has no validated Dutch population references. In the near future these reference values will be provided.

The use of a cancer-specific questionnaire (for all cancers) or a specific-cancer questionnaire (for one type of cancer) is to monitor the reactions of patients to cancer and cancer treatment. The Rotterdam Symptom Checklist is a suitable cancer-specific instrument to be used in economic evaluation. However, this questionnaire is mainly applied in patients receiving standard chemo- and/or radiotherapy (De Haes et al., 1990). Patients undergoing stem cell transplantation receive high-dose chemotherapy and sometimes total body irradiation. Stem cell transplantation is a very intensive treatment modality, that will be applied in many new patient groups. The sensitivity of the Rotterdam Symptom Checklist to monitor the burden of this treatment modality to patients requires further study.

Alternatives for the Rotterdam Symptom Checklist are for example the European Organisation for Research and Treatment of Cancer Core Quality of Life Questionnaire (EORTC QLQ-C30) (Aaronson et al., 1988; Aaronson et al. 1993) and the Functional Assessment of Cancer Therapy Scale (FACT-scale, Cella et al., 1993). The QLQ-C30 is a 30-item instrument consisting of five functional scales (physical, role, emotional, social and cognitive functioning), three symptom scales (fatigue, nausea/vomiting, and pain), a global quality of life scale, and a number of single items assessing additional symptoms (dyspnoea, sleep disturbance, constipation, and diarrhoea) and perceived financial impact of the disease and its treatment. It has been demonstrated to have good validity and reliability (Aaronson et al., 1993; Anderson et al., 1993), and is currently being 
employed in a large number of clinical trials of the EORTC, other clinical trial groups, and the pharmaceutical industry. The QLQ-C30 is a core instrument that is intended to be supplemented by more specific subscales (modules) to assess aspects of quality of life of particular importance to specific subgroups of patients (Sprangers $e t$ al., 1993). A module is a set of items assessing quality of life issues not (sufficiently) covered by the core questionnaire, and considered to be relevant for the target population and the research question at hand. This set of items may refer to disease symptoms related to tumour site (e.g. breast cancer), or stage within tumour site (e.g. local $v s$ metastatic breast cancer), side-effects of treatment (e.g. chemotherapy-induced neutropenic fever), or other quality of life dimensions such as fatigue and fear of disease recurrence. At the moment, three site-specific modules have been developed, i.e. head and neck cancer (Björdal et al., 1993), breast cancer (Sprangers et al., 1992) and lung cancer (Bergman et al., 1994). The average time to complete the QLQ-C30 and a supplementary questionnaire specific to lung cancer patients was 11 minutes (Sprangers et al., 1993). Furthermore, most patients could complete the questionnaire without assistance. As the number of modules is increasing the EORTC has developed guidelines to standardise the module development process (Sprangers et al., 1993). The EORTC QLQ-C30, including the modules, is available for use on a no-cost basis for academic researchers and those working in nonprofit settings. Industry users are required to complete a licensing agreement.

The FACT-scale is a validated, 33-item scale, developed for evaluating the quality of life of patients receiving cancer treatment (Cella et al., 1993). It can easily be completed in 5 minutes, usually without assistance. The instrument is widely used in the United States, but no validated Dutch translation is available.

The EuroQol is a suitable valuation instrument to be used in economic evaluation of cancer treatments. It is a simple health status measurement instrument designed to allow valuation of health status in terms of utilities (EuroQol study group, 1990). As the EuroQol consists of 5 dimensions with 3 answer categories, 243 health states are possible. About 40 states have empirically estimated values or utilities; the other health states are estimated using a model (Van Hout and McDonnell, 1991). However, the validity of using mathematical models is questionable, as only a small number of health states have been empirically valued. An iMTA research project has been initiated to get valuations of health states from the general population. Furthermore, an organisation has been built to provide official translations of the EuroQol and a professional user guide. 
A problem in the standardisation of instruments is that different instruments are used in Europe and in the United States. For example, the Rotterdam Symptom Checklist and the EuroQol are applied in many European studies, but are rarely used in the United States and conversely the Sickness Impact Profile is often used in the United States but to a much lesser extent in Europe. Furthermore, for a number of English/American questionnaires there are no validated Dutch translations available (e.g. the Torrance's Health Status Classification (Torrance et al, 1982) and the Functional Assessment of Cancer Therapy Scale (FACT-scale, Cella et al., 1993). At the moment, there is not enough empirical data to assess decisively the comparative advantages of the several measurement and valuation instruments. However, for achieving world wide standardisation, cooperation, not only between researchers in Europe but with researchers in the United States as well, is necessary. The development of the Short-Form 36 and the EORTC QLQ-C30 and its different translations are examples of such overseas cooperations.

The administration of both a generic, a cancer specific and a valuation instrument appeared to be too much of a burden for some of the elderly patients with AML. Reasons were the duration of the questionnaire and motivation (assistance of a nurse was sometimes necessary). These problems appear specific to this aged category of patients and resulted in refusals to further cooperate in the quality of life study. For future research we would like to pay more attention to alleviate the burden of completion of the questionnaires, especially for the elderly. A solution may be to make different questionnaires for the short- and long-term quality of life measurement. It is possible to use for the shortterm quality of life measurement a cancer-specific questionnaire (e.g. the Rotterdam Symptom Checklist or the EORTC QLQ-C30) in combination with the EuroQol. Before the start of the treatment and for the long-term quality of life a generic instrument (e.g. the Nottingham Health Profile or the Short-Form 36) in combination with the EuroQol may be used.

\subsection{Measuring and valuing resources}

In economic evaluation, a distinction is made between direct medical costs, direct nonmedical costs, indirect medical costs and indirect nonmedical costs. The direct costs concern the costs of operating a treatment programme. Direct nonmedical costs are for example patient's travel costs and housekeeping costs. The indirect medical costs concern the costs of medical consumption during life years saved. The indirect nonmedical costs relate to production losses in society as a consequence of illness or treatment. The 
importance of nonmedical costs varies between treatments and patient groups. The studies in this thesis are mainly focused on direct medical costs.

In the Dutch hospitals most information systems do not contain relevant data on direct medical costs. Thus, studying patient files was the only way to collect all relevant cost data. This method of data collection is very time consuming. Inclusion of the most important cost items in case registry forms may simplify the data collection. For example, data on type of hospital care, consultations, medication, blood transfusions and outpatient visits may be added to the case registry forms. Before starting an add-on economic evaluation, the possibilities of such an inclusion may be considered.

In the near future other methods of data collection will become available. In the future hospitals will face more incentives to collect information about the input, process and output of treatment programmes. This implies that more attention will be paid to patient oriented information systems. These information systems may contain data on number of activities and unit prices relevant for economic evaluations.

Concerning the valuation of resources a clearing house for data on unit prices of the most relevant activities in cancer treatment (real prices and/or tariffs) may be helpful. Such a database necessitates a yearly update and may be used for several studies. Concerning the unit prices, the existing tariffs may be used as far as they represent the real cost price. For the most important activities separate cost price studies may have to be carried out. From the studies described in this thesis the following cost prices are available: cost of a stay on a haematological-, oncological-, isolation care- and intensive care ward per day, cost of treatment in a day care department, cost of a haematological outpatient visit, cost of bone marrow harvest, cost of the insertion of a catheter and the costs of total body irradiation (Appendix A4.3). The results of other cost price studies may be added to such database.

When comparing alternative health care programmes the pattern of costs and benefits may differ significantly between options under review. For example, health promotion (e.g. to reduce smoking by young people) and preventive health care programmes (e.g. screening for breast cancer) produce immediate costs, but benefits will only be realised many years later. ABMT may induce high treatment costs, but, if successful, it gives immediate benefits to the patient. Long-term maintenance or palliative drug therapy may have relatively low annual costs, but in spite of that it may build-up to a substantial amount over a lifetime. 
When long-term costs and consequences are taken into account, simply adding total costs and benefits over time is not adequate because people have a time preference for delaying costs and bringing forward benefits. There is a consensus that both costs and outcomes should be discounted at the appropriate rate when they are measured in monetary terms. However, there is a debate currently on-going whether non-monetary benefits, such as life years gained, should be discounted at the same rates as costs, not be discounted at all or discounted at a reduced rate. In our studies we have discounted both the costs and consequences at a discount rate of $5 \%$.

The introduction of new technologies often leads to additional costs. The introduction of ABMT for patients with haematological malignancies shows that at least savings from substitution will occur. At the moment, additional treatment modalities in these patient groups are studied. The application of PBPC transplantation seems to be a cheaper alternative to ABMT. In the near future, it is also expected that ABMT and PBPC transplantations will diffuse across other patient groups. This will lead to additional costs, but savings from substitution as well as economies of scale may occur. Scale effects are likely if care is standardised and provided on a centralised basis, as is the case with ABMT and PBPC transplantation.

\subsection{Generalisability of results}

The generalisability of our results is somewhat limited due to several reasons. Clinical trials attempt to demonstrate validly the efficacy of a technology. Therefore, a long list of inclusion and exclusion criteria is proposed and treatment guidelines are described in detail. The more specifically these criteria are defined, the better the study will be accepted from the viewpoint of reproducibility and reliability, but these criteria may drive a clinical trial far from the average clinical practice. Another aspect is the location where the trial is carried out. All studies have been carried out in a selective environment, namely in haematological intensive care hospitals. In general, such a selective environment makes it impossible to generalise study results to the average clinical practice. However, stem cell transplantations and intensive chemotherapy in patients with AML will not be carried out in each hospital and treatment protocols are rather standardised in the haematological intensive care hospitals. Thus, the costs of these treatments will hardly differ between these hospitals and therefore the results can be generalised to all haematological intensive care hospitals. 
The generalisability of the results of GM-CSF administration in patients with chemotherapy-induced fever and neutropenia may cause more problems, as these patients are also treated in regional hospitals. Treatment protocols, criteria for discharge from hospital and antibiotic regimes vary considerably between hospitals. Moreover, the prices of health care services, especially the hospital cost, will vary between the centres participating in the study and the regional hospitals. Through sensitivity analyses the impact of such variation on the cost-effectiveness of the treatment has been demonstrated.

Some problems with the generalisability of the results are not specifically related to our studies. For example, it is possible that patients participating in clinical trials may be more compliant than their counterparts in daily life. Furthermore, clinical researchers usually are more competent concerning the specific treatment issues than their colleagues in smaller hospitals. Rapid change of costs and benefits over time may invalidate conclusions by the time they appear. Moreover, generalisation of the results to other countries is not straightforward. Health care organisation and prices will differ, and valuation of health care benefits may depend on cross-cultural differences (Van Ineveld $e t$ al., 1993). In the end, the appreciation of the resulting cost-effectiveness ratio depends on the country specific opportunity cost of introducing the new technology. Therefore, in all published studies, the patient selection criteria, the treatment modalities, the methods used in the measurement and valuation of all costs and consequences must be made clear. Only then, policy makers can determine whether the study's results are applicable to their policy context, can it help other researchers to avoid duplication of research and can it facilitate the extrapolation of the study's results to other settings.

\subsection{Epilogue}

In a recent editorial in the New England Journal of Medicine, it was stated that costeffectiveness analyses are usually performed by developing a model of the outcomes of alternative treatments (Kassirer and Angell, 1994). Such a model is usually based on published data. In contrast, this thesis reports three prospective economic appraisals linked to phase III, randomised clinical trials. In our experience, these "piggyback" economic analyses in cancer research are very well feasible. 
Additional modelling has been applied, because in economic evaluation all consequences regardless of the time frame of the study should be considered. Lifelong costs and consequences will not be observed, except for patients with either complete disease-free survival or death within the study follow-up period. Therefore, modelling approaches are often required to calculate lifelong costs and consequences.

In the editorial mentioned above, it was further stated that the model specifications vary substantially. Standardisation of methods used, specification of the assumptions made and detailed presentation of costs and corresponding unit prices, will minimise the most important sources of bias. In the hospital economic model, the treatment modalities, probabilities, treatment outcomes and unit costs are described in detail and can be applied to any hospital in any country, regardless of practice patterns as hospital and/or country specific data could be used. "Piggyback" economic analyses have great potential to reduce bias due to patient selection and selection of methods when these are a priori specified in a protocol for both the clinical and economic data collection and analysis.

Another source of bias may be caused by conflict of interest. Conflict of interests may arise as professional judgements concerning a primary interest (for example patient's welfare, validity and integrity of research) tend to be unduly influenced by a secondary interest, for example financial gain or a desire for prestige and power (Thompson, 1993). However, the secondary interest is not illegimate in itself. One should prevent these secondary factors from dominating the relevant primary interest. Therefore, clinical- and economic investigators must have independence regarding methodological considerations at all stages of the study, and must have freedom of publication at all time. 


\section{4}

Summary

This thesis aims at contributing to decision making concerning the introduction of new technologies in cancer treatment through economic evaluation. Economic evaluation is a comparative analysis of alternatives in terms of both their costs and consequences. Therefore, the basic task of economic evaluation is to identify, measure, value and compare the costs and effects of the alternatives being considered. In Part I, the methodology of economic evaluation in general and of economic evaluation in cancer in specific is reviewed and practical issues are illuminated. Chapter 2 discusses basic methods of economic evaluation, the way of measuring and valuing effectiveness and resources and, finally, current practice of economic evaluation by reviewing screening and lung cancer. Chapter 3 also concerns methodological and practical issues, but it focuses on economic evaluation alongside cancer trials. In the treatment of cancer patients not only survival and disease-free survival are important outcome parameters, but also the quality of years alive. In Chapter 4 , specific attention is given to the measurement and valuation of quality of life.

Subsequently, economic evaluation is applied in several new cancer treatment modalities. In Part II the results of three retrospective cost-minimisation analyses are presented. Chapter 5 deals with autologous bone marrow transplantation (ABMT) in patients with non-Hodgkin's lymphoma (NHL) and acute leukaemia. The cost of ABMT in patients with NHL amounted to about US\$35220. Patients with acute leukaemia stayed longer in hospital and needed more supportive care. ABMT in this patient group costed about US\$ 48000 per patient.

Chapter 6 concerns patients with haematological or oncological malignancies. The patients received either ABMT with granulocyte colony-stimulating factor (G-CSF; filgrastim; Neupogen ${ }^{\circledR}$ ) or without G-CSF or alternatively, peripheral blood progenitor cell (PBPC) transplantation mobilised by G-CSF. The accelerated haematopoietic recovery in the 
PBPC group as compared with the ABMT groups, was associated with a reduction in blood transfusions, with a reduction in episodes of fever and with earlier discharge from the hospital. This resulted in the average costs per treatment of the PBPC group amounting to US\$21197 and in the ABMT groups to US\$30593 (without G-CSF) and to US\$ 32443 (with G-CSF).

In Chapter 7, the treatment costs of patients with multiple myeloma were calculated. The patients received either high-dose melphalan combined with or without G-CSF afterwards, or alternatively, PBPC mobilised by G-CSF following high-dose melphalan. Compared to the conventional treatment with melphalan, the PBPC group showed a shortening of the pancytopenic period and platelet recovery. This resulted in a cost reduction of $44 \%$ when changing to PBPC reinfusion (on average US\$32223 in the control group and US\$17908 in the PBPC group).

Part III discusses the results of three prospective cost-effectiveness studies. Chapter 8 deals with a prospective randomised clinical trial with simultaneous data collection for an economic appraisal. The study concerns patients with intermediate and high-grade malignant NHL, who attained a partial response after three cycles of chemotherapy and who had no evidence of bone marrow involvement of NHL. The standard chemotherapy group continued with cyclophosphamide, doxorubicin, vincristine and prednisone (CHOP). The ABMT group was treated with high-dose chemo-radiotherapy followed by ABMT. The overall and disease-free survival at 3 years were $61 \%$ and $60 \%$, respectively, in the ABMT group, and $85 \%$ and $77 \%$ in the CHOP group ( $P=\mathrm{NS})$. In addition, there were more (severe) complaints and symptoms in the ABMT group than in the CHOP group. Regarding treatment costs, it was shown that the average costs of CHOP chemotherapy were significantly lower than the average costs in the ABMT group (CHOP: US $\$ 3118$ versus ABMT: US $\$ 34447)$. Considering long-term consequences the ABMT group was more expensive (US\$ 34580 ) and patients experienced 0.14 life years and 0.22 quality adjusted life years less than the CHOP group (discount rate $5 \%$ ). Based on these results, changing therapy from $\mathrm{CHOP}$ to $\mathrm{ABMT}$, as primary treatment in slow responders to CHOP, should not be recommended.

Chapter 9 deals with a prospective, randomised clinical trial comparing the costs and effects of granulocyte-macrophage colony-stimulating factor (GM-CSF; molgramostim; Leucomax ${ }^{\circledR}$ ) as an adjunct to intensive chemotherapy in elderly patients with acute myeloid leukaemia (AML). The study was a combined study of the EORTC Leukaemia Group and the Dutch HOVON Haemato-Oncology Group. The patients were randomised to either daunomycin-cytosine arabinoside (control arm) or daunomycin-cytosine ara- 
binoside with GM-CSF (GM-CSF arm). GM-CSF was given to the second group of patients during chemotherapy as well as during the phase of bone marrow suppression post chemotherapy. The patients were treated with one, or in case of a partial response, with two remission induction cycles, and when a complete remission was attained, they received one additional course of consolidation therapy. Complete remission was achieved in $46.2 \%$ (after induction cycle I) and $57.9 \%$ (after cycle II) of the patients in the control group and in 50.0\% (after the first chemotherapy cycle) and 54.9\% (after cycle II) of the patients in the GM-CSF group $(P=\mathrm{NS})$. The duration of survival and disease-free survival at 2 years after randomisation were estimated at $26 \%$ and $19 \%$ for the control group and $23 \%$ and $11 \%$ for the GM-CSF group $(P=N S)$. Concerning the time of recovery of neutrophils there were significant differences in favour of the GM-CSF group. However, the administration of GM-CSF resulted in moderate to severe toxicities attributed to GM-CSF. The quality of life analysis showed more problems with regard to depressed mood, diarrhoea and rash/eczema. Considering the long-term quality of life there were no significant differences between the two groups. Considering a follow-up period of 2 years, the cumulative, discounted survival amounted to 0.979 life years for the control group and to 1.007 for the GM-CSF group. The quality adjusted survival was 0.800 for the control group and 0.816 for the GM-CSF group. The average costs of the primary treatment were higher in GM-CSF treated patients than in the control group, i.e. US $\$ 40782$ and US $\$ 34465$, respectively $(P<0.01)$. The costs during the follow-up period did not differ between the two groups. They averaged about US $\$ 11000$ during the first year of follow-up and about US $\$ 6000$ during the second year. The results of this randomised clinical trial indicate that daunomycin-cytosine arabinoside plus GM-CSF is not a cost-effective treatment strategy as compared with daunomycin-cytosine arabinoside alone.

The third study focuses on patients with chemotherapy-related fever and neutropenia (Chapter 10). Neutropenia is a frequent and serious complication of cancer chemotherapy. When present in combination with fever, neutropenia often becomes life-threatening, and requires additional health care resources. Haematopoietic growth factors (HGFs) reflect a new and effective means of treating chemotherapy patients to prevent fever and neutropenia. The use of HGFs may reduce the duration and/or severity of neutropenia. Also, the administration of HGFs may lead to significant cost savings, by reducing the stay in hospital, and may produce quality of life benefits. However, the administration of GMCSF did not produce quality of life benefits as compared with placebo. The median hospitalisation period of the patients who received GM-CSF was 6 days (mean: 7.25; range: 3 -14). The patients treated with placebo had a hospital stay of 7 days (mean: 8.33 ; range: $4-14$ ). The median total treatment costs amounted to US\$ 4140 (mean: US\$ 5180; 
range: US\$1710 - US\$ $\$ 14270$ ) in the GM-CSF group and to US\$ 3590 (mean: 4180; range: US $\$ 1680$ - US $\$ 11450$ ) in the placebo group. This difference is significant $(P<0.05)$ and mainly due to the additional cost of GM-CSF.

The implementation of ABMT and HGFs in the clinical practice may have major macroand microeconomic consequences. In Chapter 11, the extra costs of introducing ABMT in the treatment of patients with malignant lymphoma and acute myeloid leukaemia in the Netherlands are assessed. In the Netherlands, the total number of ABMTs per year in these patient groups was estimated at $230 ; 180$ in the NHL group and 50 in the AML group. The extra costs of introducing ABMT in the NHL group will vary between 4.94 and 6.68 million dollars and in the AML group these costs were estimated at 2.95 million dollars. As a result, the total extra costs of introducing ABMTs are expected to be between 7.89 and 9.63 million dollar.

The costs of introducing HGFs in the clinical practice are presented by a Markov type economic model for the hospital, which calculates all relevant direct costs and savings of HGF therapy and may support decisions on HGF administration (Chapter 12). Three categories of treatment choices are presented: a. a treatment option where HGFs are administered during intensive chemotherapy schedules, b. a prophylactic option where HGFs are administered during and after standard chemotherapy cycles, and c. HGF therapy in patients with chemotherapy induced fever and neutropenia. Our results indicate that HGFs can induce savings in intensive chemotherapy and standard chemotherapy following neutropenic fever. Prophylactic administration of HGF is cost-effective if the risk of infection is considerable. The risk of infection depends on underlying malignancy, corresponding treatment modalities and the health condition of the patient. The model is meant as an analytical framework and should be used carefully, as not all benefits (e.g. benefits to the patients) are considered. These benefits may be balanced against the additional costs or savings resulting from the economic model.

Chapter 13 concludes with a discussion and with recommendations for further research. Economic evaluation is intended to support health policy at different levels of decision making. Using results of economic evaluations in health policy requires that the methods used and the way in which costs and consequences are measured and valued must be made clear. In this respect standardisation of methods is important. Finally, issues concerning the generalisability of the study results are described. 


\section{Samenvatting}

Dit proefschrift heeft als doel een bijdrage te leveren aan besluitvorming omtrent de introductie van nieuwe kankerbehandelingen door middel van economische evaluatie. Economische evaluatie is een vergelijkende analyse in termen van kosten en opbrengsten. Hiervoor is identificatie, meting, waardering en vergelijking van de kosten en effecten noodzakelijk. In deel I wordt de methodologie van economische evaluatie in het algemeen en van kankerbehandelingen in het bijzonder uiteengezet en worden practische problemen beschreven. Hoofdstuk 2 beschrijft de verschillende methoden van economische evaluatie, welke kosten en effecten in een dergelijke evaluatie meegenomen dienen te worden, hoe de kosten en effecten gewaardeerd dienen te worden en vervolgens de toepassing bij screening en longkanker. In hoofdstuk 3 worden eveneens methodologische en practische issues behandeld. In dit hoofdstuk ligt de nadruk echter meer op economische evaluaties die gekoppeld zijn aan klinische studies. Bij het behandelen van kankerpatiënten is behalve (ziektevrije) overleving ook kwaliteit van leven een belangrijke uitkomstmaat. In hoofdstuk 4 wordt hier speciale aandacht aan geschonken.

Vervolgens is een aantal economische evaluaties van kankerbehandelingen uitgevoerd. In deel II worden de resultaten beschreven van drie retrospectieve kosten-minimeringsanalyses. Hoofdstuk 5 heeft betrekking op patiënten met non-Hodgkin's lymphoma en acute leukemie die een autologe beenmergtransplantatie (ABMT) ondergaan. De kosten van een ABMT bij patiënten met non-Hodgkin's lymphoma bedroegen US $\$ 35220$ per patiënt. De kosten van ABMT's bij acute leukemie patiënten lagen beduidend hoger. Deze patiënten lagen in het algemeen langer in het ziekenhuis en behoefden meer isolatie, meer transfusies en meer antibiotica. De kosten voor deze groep werden geschat op US\$48000.

Hoofdstuk 6 heeft betrekking op patiënten met een hematologische- of oncologische maligne aandoening. De behandelingsmodaliteiten waren ABMT met of zonder granulocyt kolonie-stimulerende factor (G-CSF; filgrastim; Neupogen ${ }^{\circledR}$ ) en perifere stamceltransplan- 
tatie (PSCT) in combinatie met G-CSF. Het hematologische herstel in de PSCT groep in vergelijking met de beide ABMT groepen, ging gepaard met een reductie in bloedtransfusies, koorts episoden en verpleegdagen. Dit resulteerde in significant lagere gemiddelde kosten in de PSCT groep. De gemiddelde kosten in de PSCT groep bedroegen US\$ 21197 , in de ABMT groep zonder G-CSF US $\$ 30593$ en in de ABMT groep met G-CSF US\$ 32443 .

In hoofdstuk 7 zijn de behandelingskosten van patiënten met multiple myeloma berekend. Deze patiënten werden behandeld met hoge dosis melphalan soms in combinatie met GCSF of met PSCT plus G-CSF na hoge dosis melphalan. De PSCT groep had in vergelijking met de conventionele behandelingsmethode een beduidend kortere pancytopenische periode en een sneller herstel van de bloedplaatjes. Dit resulteerde in een kostenreductie van $44 \%$ van PSCT ten opzichte van de conventionele behandeling met melphalan. De gemiddelde kosten in de conventionele groep bedroegen US\$ 32223 en in de PSCT groep US\$ 17908 .

Deel III behandelt de resultaten van drie prospectieve kosten-effectiviteitsstudies. Hoofdstuk 8 beschrijft een prospectief gerandomiseerd klinisch onderzoek waaraan een economische evaluatie is gekoppeld. De studie heeft betrekking op patiënten met nonHodgkin's lymphoma van hoge en intermediaire maligniteitsgraad die na drie kuren conventionele chemotherapie (CHOP) een partiële remissie hebben bereikt en wier beenmerg tumorvrij is. Het doel van deze studie is het bepalen van de kosten en effecten van $A B M T$ en intensieve chemotherapie in vergelijking met de conventionele behandeling bij patiënten lijdend aan NHL. De conventionele behandeling bestond uit cyclofosfamide, doxorubicin, vincristine en prednison (CHOP). De ABMT groep werd behandeld met hoge dosis chemo-radiotherapie gevolgd door ABMT. De drie-jaars overleving in de ABMT groep bedroeg $60 \%$ en in de CHOP groep $77 \%$. Drie jaar na de randomisatie bedroeg de ziektevrije overleving $61 \%$ in de ABMT groep en $85 \% \%$ in de CHOP groep. De verschillen zijn niet significant. In het algemeen hadden de ABMT patiënten meer klachten en symptomen dan de patiënten die met CHOP werden behandeld. Met betrekking tot de kosten van beide behandelingen was de ABMT groep significant duurder dan de CHOP groep. De ABMT groep kostte gemiddeld US $\$ 34447$ en de CHOP groep US\$ 3118. Indien een follow-up van twee jaar en een disconteringsvoet van $5 \%$ in ogenschouw worden genomen, dan was de ABMT groep gemiddeld US $\$ 34580$ per patiënt duurder dan de CHOP groep. Tevens hadden de ABMT patiënten 0.14 levensjaren en 0.22 voor kwaliteit gecorrigeerde levensjaren minder dan de CHOP patiënten. Samenvattend kan geconcludeerd worden dat de CHOP behandeling de meest kosten-effectieve behandeling is voor patiënten met non-Hodgkin's lymphoma van hoge en intermediaire maligni- 
teitsgraad die na drie kuren conventionele chemotherapie (CHOP) een partiële remissie hebben bereikt.

Hoofdstuk 9 behandelt een prospectieve, gerandomiseerde klinische studie die de kosten en effecten van granulocyt-macrofaag kolonie-stimulerende factor (GM-CSF; molgramostim; Leucomax ${ }^{\circledR}$ ) gedurende en na intensieve chemotherapie bij oudere patiënten met acute myeloïde leukemie (AML) onderzoekt. De studie was een gecombineerde studie van de EORTC Leukaemia Group en de HOVON. De patiënten werden gerandomiseerd voor daunomycine-cytosine arabinoside (controle arm) of daunomycine-cytosine arabinoside met GM-CSF (GM-CSF arm). GM-CSF werd toegediend gedurende de chemotherapie en gedurende de aplastische fase. Alle patiënten kregen één remissie inductie kuur en indien ze een partiële respons hadden twee remissie inductie kuren. Indien een complete remissie werd bereikt, kregen de patiënten een extra consolidatie kuur. Een complete remissie werd bereikt in $46.2 \%$ (na inductie kuur I) en $57.9 \%$ (na kuur II) in de controle groep en in $50.0 \%$ (na de eerste inductie kuur) en $54.9 \%$ (na kuur II) van de patiënten in de GMCSF groep $(P=\mathrm{NS})$. De twee-jaars overleving en ziektevrije overleving na de randomisatie bedroegen respectievelijk $26 \%$ en $19 \%$ in de controle groep en respectievelijk $23 \%$ en $11 \%$ in de GM-CSF groep $(P=\mathrm{NS}$ ). Met betrekking tot het hematologisch herstel waren er significante verschillen ten gunste van de GM-CSF toediening. Verder leidde de GMCSF toediening tot meer toxiciteit. De kwaliteit van leven analyse toonde meer problemen met betrekking tot neerslachtigheid, diarree en huiduitslag in de GM-CSF groep dan in de controle groep. Ten aanzien van de kwaliteit van leven op de lange termijn waren er geen verschillen tussen de beide groepen. De twee-jaars, cumulatieve overleving bedroeg 0.979 levensjaren in de controle groep en 1.007 in de GM-CSF groep. De voor kwaliteit gecorrigeerde levensjaren waren 0.800 in de controle groep en 0.816 in de GM-CSF groep (disconteringsvoet $=5 \%$ ). De gemiddelde behandelingskosten lagen hoger bij de met GM-CSF behandelde patiënten dan bij de controle groep, te weten respectievelijk US $\$ 40782$ en US $\$ 34465(P<0.01)$. De kosten gedurende de follow-up periode verschilden niet tussen de beide groepen. In het eerste jaar bedroegen de kosten van follow-up gemiddeld US $\$ 11000$ en gedurende het tweede jaar ongeveer US\$ 6000 . Geconcludeerd kan worden dat daunomycine-cytosine arabinoside plus GM-CSF geen kosten-effectieve behandeling is in vergelijking met alleen daunomycine-cytosine arabinoside bij oudere patiënten met AML.

Hoofdstuk 10 beschrijft patiënten met chemotherapie gerelateerde koorts en neutropenie. Neutropenie is een frequent voorkomend en ernstige complicatie van chemotherapie. Indien de neutropenie gepaard gaat met koorts dan kan het een levensbedreigende complicatie zijn en vereist additionele behandeling met antibiotica. Hematopoietische 
groeifactoren zijn nieuwe middelen voor chemotherapie patiënten om febriele neutropenie te voorkomen. HGF's kunnen de duur van de neutropenie verkorten. Derhalve kan HGF toediening leiden tot kostenbesparingen ten gevolge van een korter verblijf in het ziekenhuis en kan het "kwaliteit van leven" verbeteren. Het onderzoek is uitgevoerd bij patiënten met koorts en neutropenie na chemotherapie die opgenomen moesten worden voor deze complicatie. De behandeling bestond uit het geven van breedspectrum antibiotica en de hematopoietische groeifactor GM-CSF of placebo. Dit werd minimaal 4 en maximaal 14 dagen toegediend. De behandeling werd gestaakt als gedurende 48 uur de temperatuur genormaliseerd was $\left(<37.5^{\circ} \mathrm{C}\right)$ en het neutrofielen aantal gestegen was tot $1 \times 10^{9} / 1$ of hoger. Vervolgens zijn de patiënten 24 uur geobserveerd waarna ontslag volgde. Eveneens werd de behandeling met GM-CSF of placebo gestaakt als patiënten een leucocyten aantal van $10 \times 10^{9} / \mathrm{l}$ of hoger ontwikkelden. In deze situatie werd de antibiotica behandeling gecontinueerd tot het moment dat de koorts gedurende 48 uur genormaliseerd was. De mediane opnameduur bedroeg 6 dagen in de GM-CSF groep (gemiddelde: 7.25; range: 3-14) en 7 dagen in de placebo groep (gemiddelde: 8.33; range: 4-14). De mediane totale behandelingskosten bedroegen US $\$ 4140$ (gemiddelde: US $\$ 5180$; range: US\$1710 - US\$ 14270) in de GM-CSF groep en US\$ 3590 (gemiddelde: US\$ 4180; range: US\$1680 - US\$ 11450) in de placebo groep. Dit verschil is significant en in het voordeel van de placebo behandeling. Het verschil wordt echter hoofdzakelijk veroorzaakt door de additionele kosten van GM-CSF.

Deel IV beschrijft de implementatie van ABMT en HGF's. De implementatie van deze twee technologieën in de klinische praktijk kunnen belangrijke macro- en microeconomische gevolgen hebben. De extra kosten die de introductie van ABMT in de behandeling van patiënten met maligne lymphoma en acute myeloïde leukemie in Nederland met zich meebrengt, zijn berekend (Hoofdstuk 11). In Nederland worden jaarlijks ongeveer 230 ABMT's uitgevoerd: 180 bij patiënten met non-Hodgkin's lymfoma en 50 bij patiënten met acute myeloïde leukemie. De extra kosten die de introductie van ABMT bij de NHL patiënten genereren variëren tussen de 4.94 en 6.68 miljoen dollar. Deze kosten bedragen ongeveer 2.95 miljoen dollar in de AML groep. Dit resulteert in extra kosten ten gevolge van de introductie van ABMT's van 7.89 tot 9.63 miljoen guldens voor deze patiëntengroepen.

De kosten van de introductie van HGF's in de klinische praktijk worden beschreven aan de hand van een Markov-achtig ziekenhuis economisch model (Hoofdstuk 12). Dit model berekent de belangrijkste directe kosten en besparingen die ten gevolge van HGF therapie optreden en kan besluitvorming met betrekking tot de toepassing van HGF therapie ondersteunen. Hierbij wordt onderscheid gemaakt tussen drie categorieën, te weten a. een 
behandeloptie waarbij HGF's na hoge dosis chemotherapie worden toegediend, b. een profylactische behandeloptie waarbij HGF's na standaard chemotherapie worden toegediend, en c. HGF therapie bij patiënten met een febriele neutropenie. De resultaten geven aan dat HGF therapie besparingen kunnen opleveren bij patiënten die intensieve chemotherapie ontvangen en bij patiënten die chemotherapie gerelateerde koorts en neutropenie hebben. In de profylactische strategie was de HGF therapie kosten-effectief, indien de kans op koorts en neutropenie hoog is. De kans op infectie hangt onder andere af van de onderliggende maligniteit, de daarmee samenhangende behandelingsmodaliteiten en de gezondheidstoestand van de patiënt. Het model is bedoeld als analytisch kader en dient zorgvuldig gebruikt te worden, aangezien niet alle baten (denk bijvoorbeeld aan baten voor de patiënt) in acht genomen worden. Deze baten dienen afgewogen te worden tegen de additionele kosten of besparingen die het model berekent.

Hoofdstuk 13 besluit met een discussie en aanbevelingen voor toekomstig onderzoek. Economische evaluatie heeft als doel het ondersteunen van besluitvorming. Om de resultaten van economische evaluaties te kunnen gebruiken voor gezondheidszorgbeleid is het van belang dat de gehanteerde methoden ter berekening van de kosten en opbrengsten duidelijk zijn. Standaardisatie van methoden om kosten en opbrengsten te meten en te waarderen is hierbij van belang. Tot slot wordt ingegaan op de mogelijkheden en beperkingen om de resultaten van de genoemde onderzoeken te generaliseren. 
Appendix 



\begin{tabular}{ll} 
ABMT & Autologous bone marrow transplantation \\
AML & Acute myeloid leukaemia \\
Ara-C & Cytosine arabinoside \\
BM & Bone marrow \\
CBA & Cost-benefit analysis \\
CCT & Controlled clinical trial \\
CEA & Cost-effectiveness analysis \\
CHOP & Cyclophosphamide, doxorubicin, vincristine, prednisone \\
CMA & Cost-minimisation analysis \\
CR & Complete remission \\
CUA & Cost-utility analysis \\
DNR & Daunomycin \\
EE & Economic evaluation \\
G-CSF & Granulocyte colony-stimulating factor \\
GM-CSF & Granulocyte-macrophage colony-stimulating factor \\
hr & hour \\
HGF & Haematopoietic growth factor \\
HOVON & Dutch Cooperative Group on Haemato-Oncology \\
NHL & Non-Hodgkin's lymphoma \\
p & probability \\
PBPC & Peripheral blood progenitor cell \\
PSCT & Peripheral stem cell transplantation \\
PD & Progressive disease \\
PR & Partial remission \\
QALY & Quality adjusted life year \\
QoL & Quality of life \\
\hline
\end{tabular}


Appendix

RCT

se

SCT

wk

yr
Randomised clinical trial standard error

Stem cell transplantation

week

year 


\section{References}

Aaronson NK, Ahmedzai S, Bergman B, et al. The European Organisation for Research and Treatment of Cancer QLQ-C30: A quality of life instrument for use in international clinical trials in oncology. J Nat Cancer Inst 1993; 85; 365-376.

Aaronson NK, Bullinger M, and Ahmedzai S. A modular approach to quality-of-life assessmemt in cancer clinical trials. Recent results in cancer research. Springer-Verlag, Berlin 1988.

Adams ME, McCall NT, Gray DT, et al. Economic analysis in randomised controlled trials. Med Care 1992; 30: 231-238.

Advani R, Chao NJ, Horning SJ, et al. Granulocyte-macrophage colony-stimulating factor (GM$\mathrm{CSF}$ ) as an adjunct to autologous hemopoietic stem cell transplantation for lymphoma. Ann Inter Med 1992; 116: 183-189.

Anderson RT, Aaronson NK, and Wilkin D. Critical review of the international assessments of health-related quality of life. Quality Life Res 1993; 2: 369-395.

Antman KS, Griffin JD, Elias A, et al. Effect of recombinant human granulocyte-macrophage colony stimulating-factor on chemotherapy-induced myelosuppression. $N$ Engl J Med 1988; 319: 593-598.

Armitage JO. Treatment of non-Hodgkin's lymphoma. $N$ Engl J Med 1993; 328: 1023-1030.

Armitage JO, Weisenburger PD, Hutchines $\mathrm{M}$, et al. Chemotherapy for diffuse large-cell lymphoma: rapidly responding patients have more durable remissions. J Clin Oncol 1986; 4:160164. 
Ayash LJ, Elias A, Wheeler C, et al. Double dose-intensive chemotherapy with autologous marrow and peripheral blood progenitor cell support for metastatic breast cancer: a feasibility study. J Clin Oncol 1994; 12: 37-44.

Backhouse ME, Backhouse RJ, and Edey SA. Economic evaluation bibliography. Health Econ 1992; (supp): 1-235.

Baker MS, Kessler LG, Urben N, and Smucker RC. Estimating the treatment costs of breast and lung cancer. Med Care 1991; 29: 40-49.

Ball ED and Rybka WB. Autologous bone marrow transplantation for adult acute leukemia. Hematol Oncol Clin North Am 1993; 7: 201-231.

Barlogie B, Jagannath S, Dixon D, et al. High dose melphalan and granulocyte-macrophage colony-stimulating factor for refractory multiple myeloma. Blood 1990; 76: 677-680.

Beck-Friis B, Norberg $\mathrm{H}$ and Strang P. Cost analysis and ethical aspects of hospital-based homecare for terminal cancer patients. Scand J Prim Health Care 1991; 9: 259-264.

Bennett K and Torrance GW. McMaster Rheumatoid Arthritis Utility Measurement Questionnaires. McMaster University, Dept. of Clinical Epidemiology and Biostatistics, IIton (Ontario) Canada 1990.

Berg RL. Cancer prevention and screening in light of health promotion and prevention of disability for the second 50 years. A report from the Institute of Medicine of the National Academy of Science. Cancer 1991; 68S: 2511-2513.

Bergman B, Aaronson NK, Ahmedzai S, et al. The EORTC QLQ-C30: a modular supplement to the EORTC Core Quality of Life Questionnaire (QLQ-C30) for use in lung cancer clinical trials. EORTC Study' Group on Quality of Life. Eur J Cancer 1994; 30A: 635-642.

Bergner M, Bobbitt RA, Carter WB, and Gilson BS. The sickness impact profile: development and final revision of a health status measure. Med Care 1981; 19: 787-805.

Biesma B, Vries EGE de, Willemse PHB, et al. Efficacy and tolerability of recombinant human granulocyte-macrophage colony-stimulating factor in patients with chemotherapy-related leucopenia and fever. Eur J Cancer 1990; 26: 932-936.

Björdal K, Kaasa S, Ahlner-Elmqvist M, et al. Development of a head and neck cancer-specific module for use with the EORTC Core Quality of life questionnaire (EORTC QLQ-C30). Quality Life Res 1993; 2:72. 
Bodey GP, Buckley M, Sathe YS, et al. Quantitative relationships between circulating leukocytes and infection in patients with acute leukemia. Ann Intern Med 1966; 64: 328-340.

Bonsel GJ, Rutten FFH and Uyl-de Groot CA. Economic evaluation alongside cancer trials: methodological and practical aspects. Eur J Cancer 1993; 29A: S10-S14.

Bonsel GJ, Essink-Bot ML, Klompmaker IJ, et al. Assessment of the quality of life before and during liver transplantation. Transplant 1992; 53; 796-800.

Bonsel GJ, Klompmaker I, Veer F van't, et al. Use of prognostic models for assessment of value of liver transplanation. Lancet 1990; 335: 493-497.

Boyle MH, Torrance GW, Sindair JC and Horwood SP. Economic evaluation of neonatal intensive care of the very low birthweight infants. N Engl J Med 1983; 308: 1330-1337.

Brandt SJ, Peters WP, Atwater SK, et al. Effect of recombinant human granulocyte-macrophage colony-stimulating factor on haematopoeitic reconstruction after high-dose chemotherapy and autologous bone marrow transplantation. $N$ Engl J Med 1988; 318: 593-598.

Bried EM and Scheffler RM. The financial stages of cancer in the elderly. Oncology 1992; 6S: 153-160.

Broder $S$. The human costs of cancer and the response of the National Cancer Program. Cancer 1991; 67S: $1716-1717$.

Bronchud MH, Scarffe JH, Thatcher N, et al. Phase I/Il study of recombinant human granulocyte colony-stimulating factor in patients receiving intensive chemotherapy for small lung cancer. $\mathrm{Br} J$ Cancer 1987; 56: 809-813.

Brown ML and Fintor L. Cost-effectiveness of breast cancer screening; preliminary results of a systematic review of the literature. Breast Cancer Res Treatm 1993; 25: 113-118.

Brugger W, Bross $\mathrm{K}$, Frisch $\mathrm{J}$, et al. Mobilization of peripheral blood progenitor cells by sequential administration of interleukin-3 and granulocytes-macrophage colony-stimulating factor following polychemotherapy with etoposide, ifosfamide and cisplatin. Blood 1992; 5: 1193-1200.

Cairns J. Discounting health benefits: another perspective. Health Econ 1992; 1: 76-79.

Canadian Coordinating Office for Health Technology Assessment. Guidelines for economic evaluation of pharmaceuticals: Canada. CCOHTA, Ottawa 1994. 
Carlsson $\mathrm{P}$, Hjertberg $\mathrm{H}$, Jönsson B, et al. The cost of prostatic cancer in a defined population. Scand J Urol Nephrol 1989; 23: 93-96.

Cella DF, Tulsky DS, Gray G, et al. The Functional Assessment of Cancer Therapy scale: development and validation of the general measure. J Clin Oncol 1993; 11: 570-579.

Clark RA. Economic issues in screening mammography. Am J Röntgenol 1992; 158: 527-534.

Coebergh JWW. Incidence and prognosis of cancer in the Netherlands: studies based on cancer registries. Dissertation Erasmus University Rotterdam, Rotterdam 1991.

Coleman M. Chemotherapy for large-cell lymphoma: optimism and caution. Ann Intern Med 1985; 103: 140-142.

Crawford F, Ozer H, Stoller R, et al. Reduction by granulocyte colony-stimulating factor of fever and neutropenia induced by chemotherapy in patients with small-cell lung cancer. $N$ Engl J Med 1991; 325: 164-170.

Culyer AJ. The normative economics of health care finance and provision. In McQuire A, Fenn P and Mayhew $\mathrm{K}$ (eds). Providing health care, the economics of alternative systems of finance and delivery. Oxford University Press, Oxford 1991.

Delwel R, Salem M, Pellens $\mathrm{C}$, et al. Growth regulation of human acute myeloid leukemia: effects of five recombinant hematopoietic factors in a serum-free culture system. Blood 1988; 72; 1944-1949.

Department of Health. Assessing the effects of health technology: principles, practice, proposals. Department of Health, London 1992.

Desch CE, Lasala MR, Smith TJ, et al. The optimal timing of autologous bone marrow transplantation in Hodgkin's disease patients after a chemotherapy relapse. J Clin Oncol 1992; 10: 200209.

Dickenson K, Hewitt P, Mutch L, et al. Perusing the literature: comparison of Medline searching with a perinatal trials database. Controlled Clinical Trials 1985; 6: 306-317.

Dillman RO, Seagren SL, Propert KJ, et al. A randomized clinical trial of induction chemotherapy plus high dose radiation versus radiation alone in stage III non-small cell lung cancer. $N$ Engl $J$ Med 1990; 323: 940-945. 
Dombret $\mathrm{H}$, Chastang $\mathrm{C}$, Fenaux $\mathrm{P}$, et al. A controlled study of recombinant human granulocyte colony-stimulating factor in elderly patients after treatment for acute myelogenous leukemia. $N$ Engl J Med 1995; 332: 1678-1684.

Drummond MF. Cost-effectiveness guidelines for reimbursement of pharmaceuticals. Economic evaluation ready for its enhanced status. Health Econ 1992; 1: 85-92.

Drummond MF (ed). Economic Appraisal of Health Technology in the European Community. Oxford University Press, Oxford, 1987IV.

Drummond MF. Output measurement for resource allocation decisions in health care. In A McGuire, $\mathrm{P}$ Fenn and $\mathrm{K}$ Mayhew (eds). Providing health care: the economics of alternative systems of finance and delivery. Oxford University Press, Oxford 1991: 99-119.

Drummond MF, Brandt A, Luce B, et al. Standardising methodologies for economic evaluation in health care. Int J Technol Assess Health Care 1993II; 9: 26-36.

Drummond $\mathrm{MF}$ and Davies L. Economic analysis alongside clinical trials. Int J Technol Assess in Heallh Care 1991; 7: 561-573.

Drummond MF, Stoddart GL, Labelle R, et al. Health economics: an introduction for clinicians. Ann Int Med 1987III; 107: 88-92.

Drummond MF, Stoddart GL and Torrance GW. Methods for the economic evaluation of health care programmes. Oxford University Press, Oxford 1987 and 1989.

Drummond MF, Teeling-Smith $\mathrm{G}$ and Welle N. Economic evaluation in the development of medicines. Office of Health Economics, London 1987II.

Drummond MF, Torrance G and Mason J. Cost-effectiveness league tables: more harm than good? Soc Sci Med 1993I; 37: 33-40.

Dufoir T, Saux MC, Terraza B, et al. Comparative cost of allogeneic or autologous bone marrow transplantation and chemotherapy in patients with acute myeloid leukaemia in first remission. Bone Marrow Transplant 1992; 10: 323-329.

Durbin M. Bone Marrow Transplantation: economic, ethical, and social issues. Pediatrics 1988; 82: $774-783$. 
Dutch Health Executive Board. Recommendations indication autologous bone marrow transplantation. Recommendation of the Dutch Health Executive Board to the Minister of Welfare, Public Health and Culture. Amstelveen (April) 1993 (in Dutch).

Early Breast Cancer Trialists' Collaborative Group. Systemic treatment of early breast cancer by hormonal, cytotoxic or immune therapy. 133 randomised trials involving 31,000 recurrencies and 24,000 deaths among 75,000 women. Lancet 1992; 339: 1-15 and 71-85.

Eddy DM, Hasselblad V, McGivney W, and Herdee W. The value of mammography screening in women under age 50 years. JAMA 1988; 259: 1512-1519.

Eddy RJ. Cost effectiveness of CT scanning compared with mediastinoscopy in the preoperative staging of lung cancer. J Can Assoc Radiol 1989; 40:189-193.

Elixhauser A (editor). Health care cost-benefit and cost-effectiveness analysis (CBA/CEA). From 1979 to 1990: a bibliography. Med Care 1993; 31(supp):JSI-149.

Engel FL, Bergman E, and Mistiaen P. Cost-effectiveness analysis of bone marrow transplantations. Report Dutch Health Council no 17, The Hague 1987 (in Dutch).

Essink-Bot ML, Bonsel GJ and Maas PJ van der. Valuation of health states by the general public: feasibility of a standardized measurement procedure. Soc Sci Med 1990; 31: 1201-1206.

Essink-Bot ML and Rutten-van Mölken MPMH. The measurememt of health status: Inventarisation of measurement instruments for medical technology assessment. Department of Public Health/Institute for Medische Technology Assessment, Erasmus University Rotterdam, 1991 (in Dutch).

Estey E, Thall PF, Kantarjian H, et al. Treatment of newly diagnosed acute myelogenous letkemia with granulocyte-macrophage colony-stimulating factor (GM-CSF) before and during continuous-infusion high-dose ara-C + daunorubicin: comparison to patients treated without GMCSF. Blood 1992; 79: 2246-2255.

EUROQOL Study Group. EuroQoL, a new facility for the measurement of health-related quality of life. Heallh Policy 1990; 16: 147-161.

Fayers PM and Jones DR. Measuring and analysing quality of life in cancer clinical trials: a review. Stat Med 1983; 2: 429-446.

Finley RS. Measuring the cost-effectiveness of hematopoietic growth factor therapy. Cancer 1991; 67S: 2727-2730. 
Fisher RI, Gaynor ER, Dahlberg S, et al. Comparison of a standard regimen (CHOP) with three intensive chemotherapy regimens for advanced non-Hodgkin's lymphoma. $N$ Engl J Med 1993; 328: 1002-1006.

Foon KA, Zighelboim F, Yale C, et al. Intensive chemotherapy in the treatment of choice for elderly patients with acute myelogenous leukemia. Blood 1981; 58: 467-470.

Forrest and Sir P. Breast cancer screening. Report to the Health Ministers of England, Wales, Scotland and Northern Ireland, 38 Department of Health and Social Security, 1987.

Gabrilove JL, Jakubowski A, Scher $\mathrm{H}$, et al. Effect of granulocyte colony-stimulating factor on neutropenia and associated morbidity due to chemotherapy for transitional-cell carcinoma of urothelium. $N$ Engl J Med 1988; 318: 1414-1422.

Ganz PA, Schag CAC, Lee JJ and Sims MS. The CARES: a generic measure of health-related quality of life for patients with cancer. Quality of life Res 1992; 1: 19-29.

Gerard K. Cost-utility in practice: a policy maker's guide to the state of the art. Health Policy 1992; $21: 1-31$ and 249-279.

Gerhartz HH, Engelhard M, Meusers P, et al. Randomized, double-blind, placebo-controlled, phase IIl study of recombinant human granulocyte-macrophage colony-stimulating factor as adjunct to induction treatment of high-grade malignant non-Hodgkin's lymphomas. Blood 1993; 82: 2329-2339.

Glantz SA. Primer of biostatistics. McGraw-Hill Book Inc., Singapore 1989: 287-330.

Glaspy JA, Bleecker G, Crawford J, et al. The impact of therapy with filgrastim (recombinant granulocyte colony-stimulating factor) on the health care costs associated with cancer chemotherapy. Eur J Cancer 1993; 29A: S23-S30.

Goldhirsch A, Gelber RD, Simes RJ, et al. Costs and benefits of adjuvant therapy in breast cancer: a quality-adjusted survival analysis. J Clin Oncol 1989; 7: 36-44.

Goodwin PJ, Feld R, Evans WK, et al. Cost-effectiveness of cancer chemotherapy : an economic evaluation of a randomized trial in small-cell lung cancer. J Clin Oncol 1988; 6: 1537-1547.

Gordon LI, Harrington D, Andersen J, et al. Comparison of a second-generation combination chemotherapeutic regimen (m-BACOD) with a standard regimen (CHOP) for advanced diffuse non-Hodgkin's lymphoma. N Engl J Med 1992; 327: 1342-1349. 
Gorin NC, Najman A, Douay L, et al. Autologous bone marrow transplantation in the treatment of poor prognosis non-Hodgkin's lymphomas. Eur J Cancer Clin Oncol 1984; 20: 217-225.

Gorin NC, Coiffier B, Hayat M, et al. Recombinant human granulocyte-macrophage colonystimulating factor after high-dose chemotherapy and autologous bone marrow transplantation with unpurged and purged marrow in non-Hodgkin's lymphoma: a double-blind placebo-controlled trial. Blood 1992; 80:1149-1157.

Gravelle HSE et al. Breast cancer screening and health service costs. J Health Econ 1982; 1: 185-207.

Gribben JG, Goldstone AH, Linch DC, et al. Effectiveness of high-dose combination chemotherapy and autologous bone marrow transplantation for patients with non-Hodgkin's lymphoma who are still responsive to conventional-dose therapy. J Clin Oncol 1989; 7: 1621-1629.

Gulati SC and Bennett CL. Granulocyte-macrophage colony-stimulating factor (GM-CSF) as adjunct therapy in relapsed Hodgkin disease. Ann Intern Med 1992; 116: 177-182.

Haes JCJM de and Knippenberg FCE van. The quality of life of cancer patients: a review of the literature. Soc Sci Med 1985; 20: 809-817.

Haes JCJM de, Knippenberg FCE van, and Neijt JP. Measuring psychological and physical distress in cancer patients: structure and application of the Rotterdam Symptom Checklist. $\mathrm{Br} J$ Cancer 1990; 62: 1034-1038.

Hancock BW. Quality and costs in the palliative care of cancer. $B r J$ Cancer 1992; 65: 2511 2513 .

Hazenberg BPC, Leeuwen MA van, Rijswijk MH van, et al. Correction of granulocytopenia in Felty's syndrome by granulocyte-macrophage colony-stimulating factor and interleukin 3 stimulate moncyte cytotoxicity through a tumor necrosis factor-dependent mechanism. Blood 1989; 71: 672676.

Heine JA and Rothenberger DA. Cost-effective management of colon ans rectal cancer. World $J$ Surg 1991; 15: 579-604.

Herxheimer A. The Cochrane Collaboration: making the results of controlled trials properly accessible. Postgrad Med J 1993; 69: 867-868. 
Hillner BE, Smith TJ and Desch CE. Efficacy and cost-effectiveness of autologous bone marrow transplantation in metastatic breast cancer. Estimates using decision analysis awaiting clinical trial results. JAMA 1992; 267: 2055-2061.

Hörnquist JO. The concept of quality of life. Scand J Soc Med 1982; 10: 57-61.

Houston GA, Sanders JA, Griffith JE, et al. Staging of lung cancer. A cost-effectiveness analysis. Am J Clin Oncol (CCT) 1985; 8: 224-230.

Hout BA van, Bonsel GJ, Habbema JDF, et al. Heart transplantation in the Netherlands. Costs, effects and scenarios. $J$ Health Econ 1993; 12: 73-93.

Hout BA van, and McDonnell J. Estimating a parametric relation between health description and health valuation using the Euroqol instrument. Swedish Institute for Health Economics IHE Working Paper, Lund 1991: 45-59.

Houts PS, Lipton A, Harvey HA, et al. Nonmedical costs to patients and their families associated with outpatient chemotherapy. Cancer 1984; 53: 2388-2392.

Hunt SM, McEwen J and McKenna SP. Measuring health status. Croom Helm, London 1986: 241-245.

Hutton J. Cost-benefit analysis in health care expenditure decision-making. Health Economics 1992; 1: 213-216.

Ineveld BM van, Oortmarssen GJ van, Koning HJ de, et al. How cost-effective is breast cancer screening in different EC countries? Eur J Cancer 1993; 29A: 1663-1668.

Jaakkimainen L, Goodwin PJ, Pater J, et al. Counting the costs of chemotherapy in a National Cancer Institute of Canada randomized trial in non-small cell lung cancer. J Clin Oncol 1990; 8: $1301-1309$.

Jefferson TO and Demicheli V. Is vaccination against Hepatitis B efficient? A review of world literature. Health Econ 1994 (in press).

Johanessen $M$ and Jönsson B. Economic evaluation in health care: is there a rate for cost-benefit analysis. Health Policy $1991 ; 17: 1-23$.

Johnson MG and Jolly PC. Analysis of a mass colorectal cancer screening program for cost-effectiveness. Am J Surgery 1987; 15: 261-263. 
Joseph AM, Crowson TW, and Rich EC. Cost effectiveness of hemoquant versus hemoccult for colorectal cancer screening. J General Intern Med 1988; 3; 132-138.

Kahn SB, Begg CB, Mazza JJ, et al. Full dose versus attenuated dose daunorubicin, cytosine arabinoside, and 6-thioguanine in the treatment of acute nonlymphocytic leukemia in the elderly. $J$ Clin Oncol 1984; 2: 865-870.

Kaplan EL and Meier P. Nonparametric estimation from incomplete observations. $J$ Am Stat Assoc 1959; 53: 457-481.

Kassirer JP and Angell M. The Journal's policy on cost-effectiveness analyses (editorial). $N$ Engl J Med 1994; 331: 669-670.

Keating MJ, McCredie KBM, Benjamin RS, et al. Treatment of patients over 5 years of age with acute myelogenous leukemia with a combination of rubidazone and cytosine arabinoside, vincristine and prednisone. Blood 1981; 58: 584-590.

Kennedy BJ. Excessive test costs in clinical research protocols. $J$ Cancer Educ 1991; 6: 93-97.

Kessinger A, Armitage JO, Smith DM, et al. High-dose therapy and autologous peripheral blood stem cell transplantation for patients with lymphoma. Blood 1989; 74: 1260-1265.

Kesteloot K, Dutreix A, and Schueren E van der. Quality assurance procedures in radiotherapy. Economic criteria to support decision making. Im J Technol Assess Health Care 1993I; 9: 274285.

Kesteloot K, Dutreix A, and Schueren E van der. A model for calculating the costs of in vivo dosimetry and portal imaging on radiotherapy departments. Radiother Oncol 1993II; 28: 108-117.

Koning HJ de, Ineveld BM van, Oortmarssen GJ, et al. Breast cancer screening and costeffectiveness; policy alternatives, quality of life considerations and the possible impact of uncertain factors. Int $J$ Cancer 1991; 49: 531-537.

Koopmanschap MA, Roijen L van, and Bonneux L. Cost of illness in the Netherlands. Department of Public Health / Institute for Medical Technology Assessment, Erasmus University, Rotterdam 1991 (in Dutch).

Koopmanschap MA, Roijen L van, Bonneux L, et al. Cost of diseases in international perspective. Eur J Public Health 1994; 4: 258-264. 
Koopmanschap MA and Ineveld BM van. Towards a new approach for estimating indirect costs of disease. Soc Sci Med 1992; 34: 1005-1010.

Lansky SB, List MA, Ritter-Sterr C, et al. Performance parameters in head and neck patients. Proc Am Soc Clin Oncol 1988; 7: 156 (abstract 603).

Lau J, Antman EM, Jimeneq-Silva J, et al. Cumulative meta-analysis of therapeutic trials for myocardial infarction. $N$ Engl J Med 1992; 327: 248-254.

Laupacis A, Feeny, D, Detsky AS, et al. How attractive does a new technology have to be to warrant adoption and utilization? Tentative guidelines for using clinical and economic evaluations. Can Med Assoc J 1992; 146: 473-481.

Levine MN, Guyatt GH, Gent M, et al. Quality of life in stage II breast cancer: an instrument for clinical trials. J Clin Oncol 1988; 6: 1798-1810.

Lieschke GJ and Burgess AW. Granulocyte colony-stimulating factor and granulocyte-macrophage colony-stimulating factor. $N$ Engl $J$ Med 1992; 327: 28-35.

Liu Yin JA. Acute myeloid leukemia in the elderly: biology and treatment. Brit J Haemat 1993; 83: $1-6$.

Liu Yin JA, Johnson PRE, Davies JM, et al. Mitozantrone and cytosine arabinoside as first-line therapy in elderly patients with acute myeloid leukemia. Brit J Haemat 1991; 79: 415-420.

Llewellyn-Thomas HA, Sutherland HJ, Hogg SA, et al. Linear analogue self-assessment of voice quality in laryngeal cancer. $J$ Chron Dis 1984; 37: 917-924.

Lobo PJ, Powles RL, Hananrahan A, et al. Acute myeloblastic leukaemia - a model for assessing value for money for new treatment programs. Br Med J 1991; 302: 323-326.

Lokhorst HM, Meuwissen OJATh, Verdonck LF, et al. High-risk myeloma treated with high-dose melphalan. J Clin Oncol 1992; 10: 47-51.

Löwenberg B, et al. A controlled study of granulocyte-macrophage colony-stimulating factor during and after initial chemotherapy in adults with newly diagnosed acute myelogenous leukemia. Results HOVON-4A study, 1995 (unpublished). 
Löwenberg B, Zittoun R, Kerkhofs $\mathrm{H}$, et $\mathrm{al}$. On the value of intensive remission-induction chemotherapy in elderly patients of $65+$ years with acute myeloid leukemia: a randomized phase III study of the European Organization for Research and Treatment of Cancer Leukemia Group. $J$ Clin Oncol 1989; 7: 1268-1274.

Lowson KV, Drummond MF, and Bishop JM. Costing new services: long-term domiciliary oxygen therapy. Lancet 1981; 1: 1146-1149.

Luce B and Elixhauser A. Standard for socioeconomic evaluation of health care products and services. Springer-Verlag, Berlin 1990.

Ludbrook A. Cost-effectiveness analyses of the treatment of chronic renal failure. Applied Econ 1981; 13: 337-350.

Maas PJ van der, Koning HJ de, Ineveld BM, et al. The cost-effectiveness of breast cancer screening. Int J Cancer 1989; 43: 1055-1060.

Maher DW, Lieschke GJ, Green M, et al. Filgrastim in patients with chemotherapy-induced febrile neutropenia. Amn Intern Med 1994; 121: 492-501.

Mandelblatt JS, Fahs MC. The cost-effectiveness of cervical cancer screening in low-income elderly women. JAMA 1988; 259: 2409-2413.

Martens LL, Rutten FFH, Erkelens DW, et al. Cost-effectiveness of cholesterol lowering therapy in the Netherlands: simvastatin versus cholestyramine. Am J Med 1989; 87: 4A/54S-4A/58S.

Mason J, Drummond MF, Torrance G. Some guidelines on the use of cost-effectiveness league-tables. $\operatorname{Br~Med~} J$ 1993; 306: 570-572.

Mayordomo FI, Rivera F, Diaz-Puente MT, et al. Decreasing morbidity and cost of treating febrile neutropenia by adding G-CSF and GM-CSF to standard antibiotic therapy: results of a randomized trial. Proc Am Soc Clin Oncol 1993, abstract 1510.

McDowell I and Newell C. Measuring Health - a guide to rating scales and questionmaires. Oxford University Press, Oxford 1987.

McHorney CA, Ware JE, Rogers W, et al. The validity and relative precision of MOS short- and long-form health status scales and Dartmouth COOP charts. Med Care 1992; 30: MS253-MS265. 
Medical Insurance Board. Proposal concerning patient groups for autologous bone marrow transplantation. Advice Medical Insurance Board to the Minister of Health, Welfare and Culture, Amstelveen 1993 (in Dutch).

Metcalf D. The colony stimulating factors. Discovery, development, and clinical applications. Cancer 1990; 65: 2185-2195.

Mishan EJ. Cost-benefit analysis, George Allen \& Unwin, London 1984.

Mooney G. Breast cancer screening : A study in cost-effectiveness analysis. Soc Sci Med 1982; 16: 1277-1283.

Mugford M, Jefferson TO and McGuire A. How can we generalize from 40 results of economic evaluations? Paper presented at the United Kingdom Health Economics Study Group 1994 (unpublished).

Munoz E, Chalfin D, Sterman H, et al. Hospital costs, cancer patients, and surgical diagnostic related groups. J Surg Oncol 1989; 41: 47-51.

National Cancer Institute sponsored study of classification of non-Hodgkin's lymphomas. Summary and description of a Working Formulation of clinical usage. The non-Hodgkin's lymphoma Pathologic Classification Project. Cancer 1982; 49: 2112-2135.

Nemunaitis J, Rabinowe SN, Singer JW, et al. Recombinant granulocyte-macrophage colonystimulating factor after autologous bone marrow transplantation for lymphoid cancer. $N$ Engl $J$ Med 1991; 324: 1773-1778.

Obuko I, Glick H, et al. Cost-effectiveness of mass screening for breast cancer in Japan. Cancer 1991; 67: 2021-2029.

Ohno R, Tomonaga M, Kobayashi T, et al. Effect of granulocyte colony-stimulating factor after intensive induction therapy in relapsed or refractory acute leukemia. $N$ Engl J Med 1990; 323: $871-878$.

Ossenkoppele GJ, Jonkhoff AR, Huijgens PC, et al. Peripheral blood progenitors mobilised by GCSF (filgrastim) and reinfused as unprocessed autologous whole blood shorten the pancytopenic period following high-dose melphalan in multiple myeloma. Bone Marrow Transpl 1994; 13: 3741 . 
Parkin DM and Moss SM. An evaluation of screening policies for cervical cancer in England and Wales using a computer simulation model. J Epid Comm Health 1986; 40: 143-153.

Parsonage, $M$ and Nuberger H. Discounting Health Benefits. Health Econ 1992; 1: 71-75.

Patrick DL and Deyo RA. Generic and disease-specifc measures in assessing health status and quality of life. Med Care 1989; 27: S217-S232.

Pettengell R, Gurney H, Radford JA, et al. Granulocyte colony-stimulating factor to prevent doselimiting neutropenia in non-Hodgkin's lymphoma: a randomized controlled trial. Blood 1992; 80: $1430-1436$.

Philip T, Armitage JO, Spitzer G, et al. High-dose therapy and autologous bone marrow transplantation after failure of conventional chemotherapy in adults with intermediate-grade or high-grade non-Hodgkin's lymphoma. N Engl J Med 1987; 316: 1493-1498.

Philip T, Ladenstein R, Zucker JM, et al. Double megatherapy and autologous bone marrow transplantation for advanced neuroblastoma: the LMCE2 study. Cancer 1993; 67: 119-127.

Philips GL, Herzig RH, Lazarus HM, et al. Treatment of resistant malignant lymphoma with cyclophosphamide, total body irradiation, and transplantation of cryopreserved autologous bone marrow. $N$ Engl J Med 1984; 310: 1557-1561.

Philips M, Richards S and Chessells J. Acute myeloid leukaemia in childhood: the costs and benefits of intensive treatment. Br J of Haematol 1991; 77: 473-477.

Pizzo PA, Robichaud KJ, Gill FA, et al. Duration of empiric antibiotic therapy in granulocytopenic patients with cancer. Am J Med 1976; 67: 194-200.

Pizzo PA, Robichaud KJ, Gill FA, et al. Empiric antibiotic and antifungal therapy for cancer patients with prolonged fever and granulocytopenia. Am J Med 1982; 72: 101-111.

Pizzo PA. Granulocytopenia and cancer therapy. Past problems, current solutions, future challenges. Cancer 1984; 54: 2649-2661.

Preisler HD, Rustum Y, Henderson ES, et al. Treatment of acute non-lymphocytic leukemia: Use of anthracycline-cytosine arabinoside induction therapy and comparison of two maintenance regimens. Blood 1979; 53: 455-464.

Rai KR, Holland JF, Glidewell OJ, et al. Treatment of acute myelocytic leukemia: a study by Cancer and Leukemia Group B. Blood 1981; 58; 1203-1212. 
Rees GJG. Cost-effectiveness in oncology. Lancet 1985; ii: 1405-1408.

Richel DJ. Peripheral stem cell transplantation opens the way to new treatment strategies. ECC Newsletter 1992: 55-56.

Richel DJ, Baars JW, Wijngaarden MJGJ, et al. Favourable effect of haematopoietic stem cells isolated from blood on haematological recovery after high-dose chemotherapy. Ned Tijdschr Geneeskd 1993; 137: 245-250.

Riikonen P, Saarinen UM, Mäkipernaa A, et al. Recombinant human granulocyte-macrophage colony-stimulating factor in the treatment of febrile neutropenia: a double blind placebo controlled study in children. Pediatr Inf Dis J 1994; 13: 197-200.

Rosser R and Kind P. A scale of valuations of states of illness: is there a social consensus? Int $J$ Epidemiol 1978; 7 : 347-358.

Rutten FFH, Ineveld BM van, Ommen $\mathrm{R}$ van, et at. Cost calculation concerning health care research: recommendations for general practice. Van Arkel, Utrecht 1993 (in Dutch).

Rutten FFH and Bonsel GJ. High cost technology in health care: a benefit or a burden? Soc Sci Med 1992; 35: 567-577.

Sacks H, Chalmers RC, and Smith H. Randomised versus historical controls for clinical trials. $A m$ $J$ Med 1982; 72: 33-240.

Salles G, Shipp MA and Coiffier N. Chemotherapy of non-Hodgkin's aggressive lymphoma's. Sem Hematol 1994; 31: 46-49.

Schag CAC, Ganz PA and Heinrich RLH. CAncer Rehabilitation Evaluation System - Short Form (CARES-SF). Cancer 1991; 68: 1406-1414.

Schipper H, Clinch J, McMurray A, and Levitt M. Measuring the quality of life of cancer patients. The functional living index-cancer: development and validation. $J$ Clin Oncol 1984; 2 : 472.

Selby PJ, McElwain TJ, Nandi AC, et al. Multiple myeloma treated with high dose intravenous melphalan. BrJ Haematol 1987; 66: 55-62.

Shapiro S. Periodic breast cancer screening in seven foreign countries. Cancer 1992; 69S: 19191924. 
Siena S, Bregni M, Brando B, et al. Circulation of $\mathrm{CD} 34^{+}$haematopoietic stem cells in the peripheral blood of high-dose cyclophosphamide-treated patients: enhancement by intravenous recombinant human granulocyte-macrophage colony-stimulating factor. Blood 1989; 74: 19051914.

Singer CRJ and Goldstone AH. Clinical studies of autologous bone marrow transplantation in nonHodgkin's lymphoma. Clin Haematol 1986; 15: 105-150.

Skrabanek P. The cost-effectiveness of breast cancer screening. Int J Technol Assess Health Care 1991; 7: 633-635.

Sprangers MA and Aaronson NK. The role of health care providers and significant others in evaluating the quality of life of patients with chronic disease: a review. J Clin Epidemiol 1992; 45: 743-760.

Sprangers MA, Cull A, Björdal K, et al. The European Organisation for Research and Treatment of Cancer approach to quality of life assessment: guidelines for developing questionnaire modules. Quality Life Res 1993; 2: 297-303.

Sprangers MA, Groenvold M, Velde A te, et al. for the EORTC study group on quality of life. The construction of a breast cancer module. Amsterdam 1992, internal report.

Stewart AL, Hays RD and Ware JE. The MOS Short-form General Health Study - reliability and validity in a patient population. Med Care 1988; 26: 724-735.

Stewart LA and Parmar, MKB. Meta-analysis of the literature or of individual patient data: is there a difference? Lancet $1993 ; 31: 418-422$.

Stone RM, Berg DT, George GL, et al. Granulocyte-macrophage colony-stimulating factor after initial chemotherapy for elderly patients with primary acute myelogenous leukemia. $N$ Engl J Med 1995: 332: 1671-1677.

Thompson DF. Understanding financial conflicts of interest. N Engl J Med 1993; 329: 573-576.

Thürmann $\mathrm{P}$ and Harder S. Determinants of hospital drug expenditures in Western Europe. PharmacoEconomics 1993; 4: 157-161.

Tiegs G, Barsig J, Matiba B, et al. Potentiation by granulocyte-macrophage colony-stimulating factor of lipopolysaccharide toxicity in mice. J Clin Invest 1994; 9: 2616-2623. 
Torrance GW. Measurement of health state utilities for economic appraisal: a review. $J$ Health Econ 1986; 5: 1-30.

Torrance GW. Utility approach to measuring health-related quality of life. J Chron Dis 1987; 40: 593-600.

Torrance GW, Boyle MH and Horwood SP. Application of multiattribute utility theory to measure social preferences for health states. Oper Res 1982: 1043-1069.

Trillet-Lenoir V, Green J, Manegold C, et al. Recombinant granulocyte colony-stimulating factor reduced the infectious complications of cytotoxic chemotherapy. Eur J Cancer 1993; 29A: 319324.

Turk J, Walker A, Whynes DK, et al. Screening and the costs of treating colorectal cancer: some preliminary results. Public Health 1989; 103: 413-419.

Udvarhelyi S, Colditz GA, Rai A, and Epstein AM. Cost-effectiveness and Cost-benefit analyses in the medical literature. Are methods being used correctly? Ann Intern Med 1992; 116: 238-44.

Uyl-de Groot CA, Ossenkoppele GJ, Riet AAPM van der, and Rutten FFH. Cost analysis of peripheral blood progenitor cell reinfusion mobilised by G-CSF (filgrastim) following high dose Melphalan as compared with high dose Melphalan therapy in multiple myeloma. Eur $J$ Cancer 1994I; 30A: 457-459.

Uyl-de Groot CA, Richel DJ, and Rutten FFH. Peripheral blood progenitor cell transplantation mobilised by r-metHuG-CSF (filgrastim); a less costly alternative to autologous bone marrow transplantation. Eur J Cancer 1994If; 30A: 1631-1635.

Uyl-de Groot CA, Rutten FFH, and Bonsel GJ. Measurement and valuation of quality of life in economic appraisal of cancer treatment. Eur J Cancer 1994III; 30A: 111-117.

Uyl-de Groot CA, Hagenbeek A, Verdonck LF, et al. Cost-effectiveness of autologous bone marrow transplantation in comparison with $\mathrm{CHOP}$ chemotherapy in patients with intermediate- and high grade malignant non-Hodgkin's lymphoma (NHL). Bone Marrow Transpl (in press).

Velasquez WS, Canbaillas F, Salvador P, et al. Effective salvage therapy for lymphoma with cisplatin in combination with high-dose Ara-C and dexamethasone (DHAP). Blood 1988; 71: 117 122. 
Verdonck LF, Dekker AW, Gast GC, et al. Salvage therapy with ProMace-MOPP followed by intensive chemoradiotherapy and autologous bone marrow transplantation for patients with nonHodgkin's lymphoma who failed to respond to first-line CHOP. J Clin Oncol 1992; 10: 19491954.

Verdonck LF, Dekker AW, Kempen ML van, et al. Intensive cytotoxic therapy followed by autologous bone marrow transplantation for non-Hodgkin's lymphoma of highngrade malignancy. Blood 1985; 65: 984-989.

Verdonck LF, Putten WLJ van, Hagenbeek A, et al. Comparison of CHOP chemotherapy with autologous bone marrow transplantation for slowly responding patients with aggressive nonHodgkin's lymphoma. $N$ Engl J Med 1995; 332: 1045-1051.

Ware JE. Standards for validating health measures: definition and content. J Chron Dis 1987; 40: 437-480.

Ware JE. SF-36 health survey. Manual \& interpretation guide. The Health Institute, New England Medical Center, Boston Massachusetts, 1993.

Weber RJ. Pharmaeconomic issues in the use of granulocyte-macrophage colony-stimulating factor for bone marrow transplantation or chemotherapy-induced neutropenia. Clin Ther 1993; 15: 180191.

Welch HG and Larson EB. Cost-effectiveness of bone marrow transplantation in acute nonlymphocytic leukaemia. N Engl J Med 1989; 321: 807-812.

Weltens C, Kesteloot K, Vanderelde R, et al. Comparison of plastic and Orfit masks for patient head fixation during radiotherapy; Precision and costs. Int $J$ Radiation Oncol Biol Phys (submitted).

Williams AH. The Economics of Coronary Artery by-pass Grafting. Br Med $J$ 1985; 291; 326-329.

Yarbro JW. Changing cancer care in the 1990s and the cost. Cancer 1991; 67S: 1718-1727.

Yates J, Glidewell $\mathrm{O}$, Wiernik $\mathrm{P}$, et al. Cytosine arabinoside with daunorubicin or adriamycin for therapy of acute myelocytic leukemia: a CALBG study. Blood 1982; 60:454-463. 


\section{Quality of life questionnaires}

Quality of life questionnaires:

\section{A.3.1 Nottingham Health Profile}

\section{A.3.2 EuroQol}

A.3.3 Rotterdam Symptom Checklist

\section{A.3.1 Nottingham Health Profile}

Listed below are some problems which people may have in their daily life. Look down the list and put a tick in the box under yes for any problem you experienced during the last week. Tick the box under no for any problem you didn't experience. The problems may resemble each other, but there are different nonetheless. Will you please answer every question. If you are not sure whether to say yes or no, tick whichever answer you think is more true at the moment.

$\begin{array}{llcc} & & \text { Yes } & \text { No } \\ 1 & \text { I'm tired all the time } & \square & \square \\ 2 & \text { I have pain at night } & \square & \square \\ 3 & \text { Things are getting me down } & \square & \square \\ 4 & \text { I have unbearable pain } & \square & \square \\ 5 & \text { I take tablets to help me sleep } & \square & \square \\ 6 & \text { I've forgotten what it's like to enjoy myself } & \square & \square \\ 7 & \text { I'm feeling on edge } & \square & \square \\ 8 & \text { I find it painful to change position } & \square & \square\end{array}$


I feel lonely

10 I can only walk about indoors

11 I find it hard to bend

12 Everything is an effort

13 I'm waking up in the early hours of the morning

14 I'm unable to walk at all

15 I'm finding it hard to make contact with people

16 The days seem to drag

17 I have trouble getting up and down stairs and steps

18 I find it hard to reach for things

19 I'm in pain when I walk

20 I lose my temper easily these days

21 I feel there is nobody I am close to

22 I lie awake for most of the night

$23 \quad 1$ feel as if I'm losing control

24 I'm in pain when I'm standing

25 I find it hard to dress myself

26 I soon run out of energy

27 I find it hard to stand for long

(e.g. at the kitchen sink, waiting for a bus)

28 I'm in constant pain

29 I feel I am a burden to people

30 It takes me a long time to get to sleep

31 Worry is keeping me awake at night

32 I feel that life is not worth living

33 I sleep badly at night

34 I'm finding it hard to get on with people 
Yes No

35 I need help to walk about outside

(e.g. a walking aid or someone to support me)

$36 \quad$ I'm in pain when going up and down stairs or steps

$37 \quad$ I wake up feeling depressed

$38 \quad$ I'm in pain when l'm sitting

Now we would like you to think about the activities in your life which may be affected by health problems.

In the list below, tick yes for each activity in your life which is being affected by your state of health. Tick no for each activity which is not being affected.

When you are not sure or when you do not want to answer the question...please tick the box under the question remark...("?")

Is your present state of health causing problems with your...

Yes ? No

39 Job or work (that is, paid employment)

40 Looking after the home (examples: cleaning \& cooking, repairs, odd jobs around the home)

41 Social life

42 Home life (that is, relationships with other people in your home)

43 Sex life

44 Interests and hobbies (sports etc.)

45 Holidays

\section{A.3.2 EuroQol descriptive part}

By placing a tick in one box in each group below, please indicate which statements best describe your own health state during the last week. 
46 Mobility

I have no problems in walking about

I have some problems in walking about

I am confined to bed

47 Self-care

I have no problems washing or dressing myself

I have some problems washing or dressing myself

I am unable to wash or dress myself

48 Usual activities (e.g. work, study, housework,

family or leisure activities)

I have no problems with performing my usual activities

I have some problems with performing my usual activities

I am unable to perform my usual activities

49 Pain/Discomfort

I have no pain or discomfort

I have moderate pain or discomfort

I have extreme pain or discomfort

50 Anxiety/Depression

I am not anxious or depressed

I am moderately anxious or depressed

I am extremely anxious or depressed

\section{A.3.3 Rotterdam Symptom Checklist}

In the list below you will be asked about your symptoms. Would you please, for all symptoms mentioned, indicate to what extent you have been bothered by it, by circling the answer most applicable to you. The questions are related to the past week.

Have you, during the past week, been bothered by

Not at all A little Quite a bit Very much

51 Lack of appetite

52 Irritability

53 Tiredness 
Not at all A little Quite a bit Very much

54 Worrying

$\square$

$\square$

55 Sore muscles

56 Depressed mood

57 Lack of energy

58 Low back pain

59 Nervousness

60 Nausea

61 Desperate feelings about the future

62 Difficulty sleeping

63 Headaches

64 Vomiting

65 Dizziness

66 Decreased sexual interest

67 Tension

68 Abdominal aches

69 Anxiety

70 Constipation

71 Diarrhoea

72 Shivering

73 Tingling hands or feet

74 Difficulty concentrating

75 Sore mouth/ pain when swallowing

76 Loss of hair

77 Burning/sore eyes

78 Shortness of breath 


$\begin{array}{llcccc} & \text { Not at all } & \text { A little } & \text { Quite a bit } & \text { Very much } \\ 79 & \text { Dry mouth } & \square & \square & \square & \square \\ 80 & \text { Constipation } & \square & \square & \square & \square \\ 81 & \text { Painful joints } & \square & \square & \square & \square \\ 82 & \text { Palpitations* } & \square & \square & \square & \square \\ 83 & \text { Rash, eczema* } & \square & \square & \square & \square \\ 84 & \text { Sweating, perspiring } & \square & \square & \square & \square\end{array}$

Finally, a number of activities is listed below. We do not want to know whether you actually do these, but only whether you are able to perform them presently. Would you please mark the answer that applies most to your condition of the past week.

$\begin{array}{cc}\text { only with } & \text { without without help } \\ \text { help } & \text { help, } \\ & \text { with } \\ \text { difficulty }\end{array}$

85 Care for myself (wash etc.)

86 Walk about the house

87 Housekeeping

88 Climb stairs

89 Odd jobs

90 Walk out of doors

91 Shopping

92 Go to work

Would you please check whether you answered all questions?

Thank you for your help. 


\section{A4}

Unit prices

A4.1 Costs of the pre-transplantation period (in US\$)

\begin{tabular}{|l|r|}
\hline Pre-transplantation period & Costs \\
\hline BM harvest & \\
- Personnel & 689 \\
- Operating room & 543 \\
- Medical equipment & 436 \\
- Materials & 222 \\
- Quality control BM & 152 \\
Total BM harvest & 2042 \\
& \\
Hospitalisation & 1367 \\
Visits & 98 \\
Laboratory services & 691 \\
Diagnostics & 714 \\
Medication & 420 \\
Transfusions & 149 \\
Total pre-transplantation & 5481 \\
\hline
\end{tabular}

A4.2 Costs of total body irradiation (in US\$)

\begin{tabular}{|l|r|}
\hline TBI & Costs \\
\hline Per session (10 minutes) & \\
- Personnel & 81 \\
- Medical equipment & 26 \\
- Other capital expenditures & 12 \\
- Exploitation & 28 \\
Subtotal & 147 \\
TBI =6 sessions (about I hour) & 882 \\
\hline
\end{tabular}


A4.3 Unit prices (in US\$)

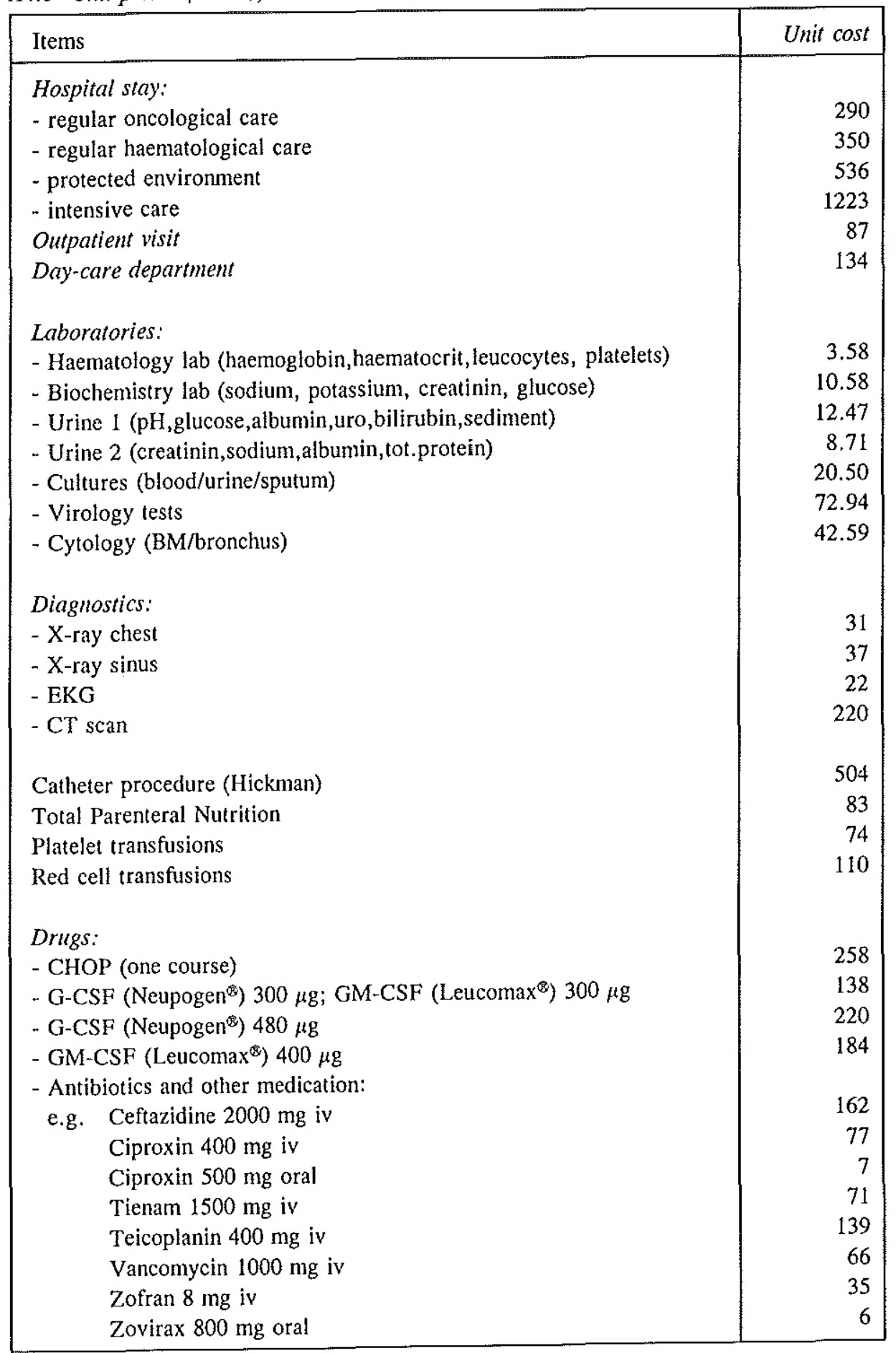




\section{Acknowledgements}

In science you never walk alone. The number of people I have met on the road to this thesis is large and many of them contributed significantly to the work presented. I would like to thank them all for helping me with this book.

I wish to acknowledge many persons for their crucial contribution to this thesis. I am most indebted to my promotor Frans Rutten for his stimulating and critical comments on the drafts and for the inspiring cooperation in the different research projects. I am also grateful to my colleagues of the institute for Medical Technology Assessment, in particular Aat van Klooster-Rutteman, Ivonne Buijt and Angélique Visser, to colleagues in the field of cancer, especially Bob Löwenberg, Peter Huijgens and Edo Vellenga, to the research nurses and datamanagers in the participating hospitals, and above all my family and friends who inspired me and spent with me many pleasant moments,

Some persons will not be aware of their stimulating role: the patients participating in the several studies, the authors of articles and books which provided insight into the subject, and the unknown referees who reviewed earlier drafts and provided many helpful comments. 
